\title{
Ende des neuseeländischen Kinos?
}

\author{
Eine kulturmaterialistische, filmgeografische Studie \\ zur Bedeutung von Nation und location \\ im Kontext der Globalisierung
}

\author{
Dissertation \\ zur Erlangung des philosophischen Doktorgrades \\ an der Philosophischen Fakultät der Georg-August-Universität Göttingen
}

vorgelegt von

Oliver Steinert-Lieschied

aus Hannover

Göttingen 2016 



\section{Danksagung}

Die Verfassung einer Dissertation gleicht einer langen Reise auf unbekanntem und gelegentlich schwierigem Terrain. Bei allen Personen, die mich auf dieser Reise begleitet haben, möchte ich mich auf diesem Wege bedanken.

Mein besonderer Dank gilt meinen Eltern Bernd und Olga Steinert-Lieschied sowie meinem verstorbenen Opa Herold Steinert-Lieschied. Euch ist diese Arbeit gewidmet.

Vielen herzlichen Dank auch an Frau Prof. Schaff für die gute Betreuung und die hilfreichen Anregungen. Ferner danke ich Herrn Prof. Haekel und Frau Prof. Bendix für die Zweit- bzw. Drittbegutachtung.

Last but not least, vielen lieben Dank an Thilo, meine Schwester und Martina für die vielen Diskussionen um inhaltliche und sprachliche Aspekte meiner Arbeit sowie H. R.'s unermüdliche Begeisterung für „semiotic landscapes“. 



\section{Inhaltsverzeichnis}

Inhaltsverzeichnis .

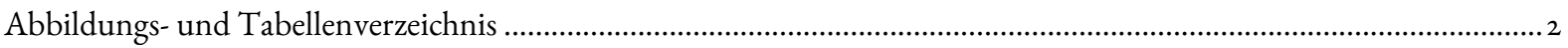

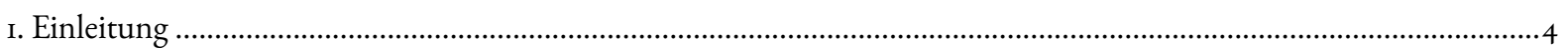

2. Konstruktion und De-/Renationalisierung des neuseeländischen Kinos im Kontext der Globalisierung .....................

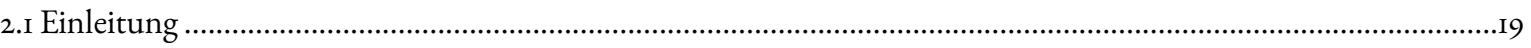

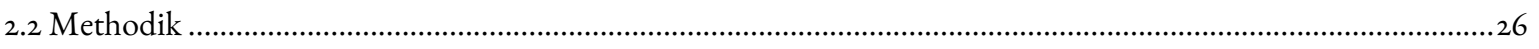

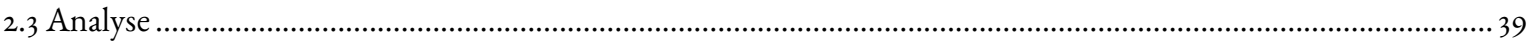

2.4 Überlegungen zur De-/Renationalisierung filmischer Inhalte...........................................................................69

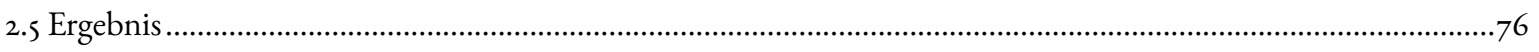

3. De-/Entlokalisierung der neuseeländischen location und dessen Bedeutung für die Frage nach filmgeografischer

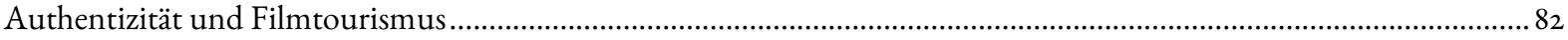

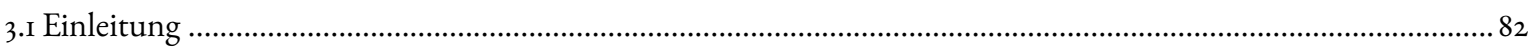

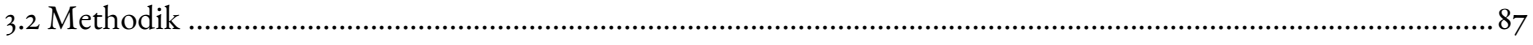

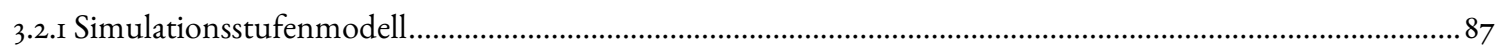

3.2.2 Authentizitätsbegriff und filmgeografische Authentizität .......................................................................94

3.2.3 Authentisierungsstrategien der Filmemacher und Authentizitätserwartungen der Zuschauer.....................97

3.2.3.I III. + IV. Simulationsstufe: Filmgeografische Delokalisierung als Authentisierungsstrategie ................97

3.2.3.2 V. + VII. Simulationsstufe: Archaisch-mythische Ästhetik, (Hyper-)Sublimität, Fotorealismus als

Authentisierungsstrategien und Entlokalisierungsprozess …….............................................................. 106

3.2.3.3 Rolle der Para- und Metatexte im Authentizitätsdiskurs ......................................................................... III

3.2.3.4 „Sehnsuchtsgrad“, location-Vermittlung, „Referenzialitätsgrad“ als Faktoren bei der Generierung von

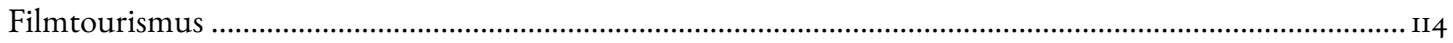

3.3 Überlegungen zu Authentizität, De- und Entlokalisierung in I. + II. Simulationsstufe ..................................... II7

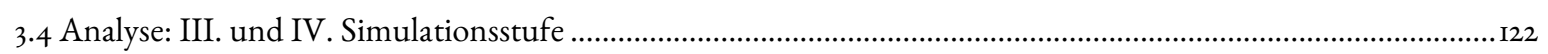

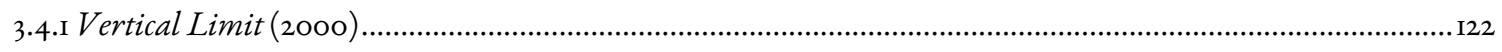

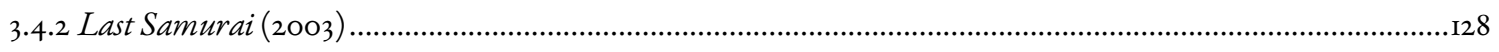

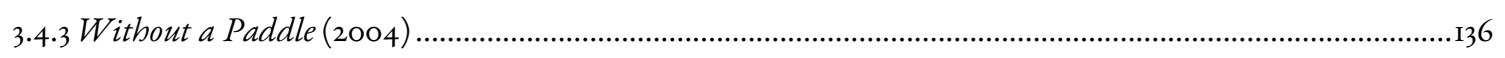

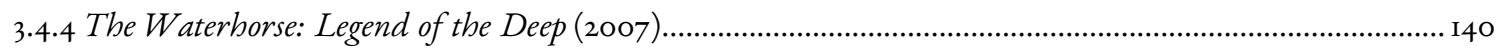

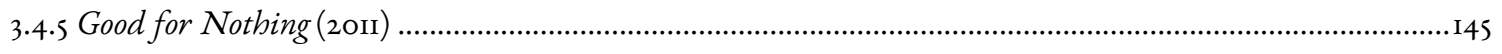

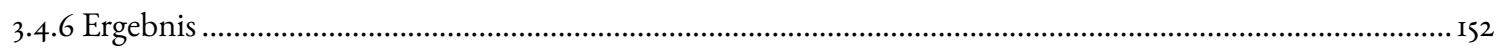

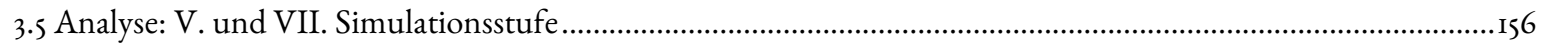

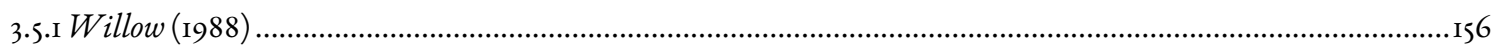

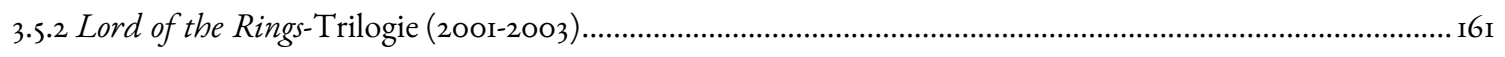

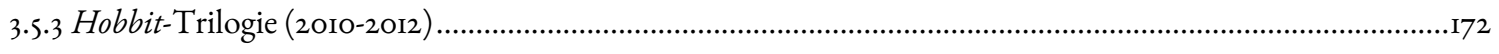

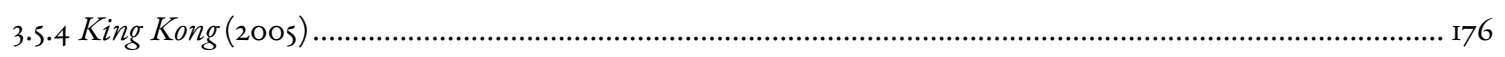

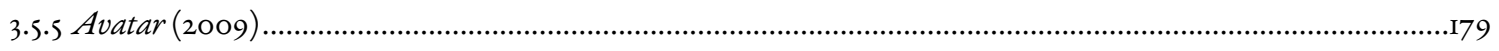

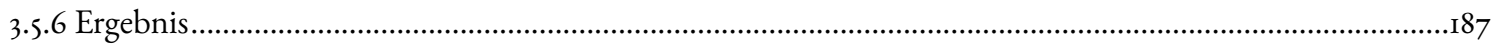

4. Ausblick und offene Forschungsfragen/Anwendungsbereiche …..................................................................

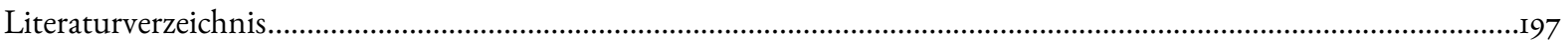




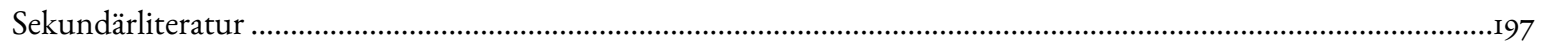

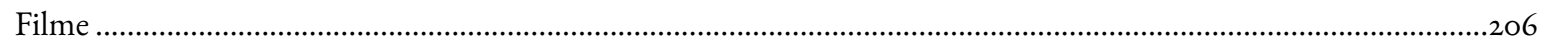

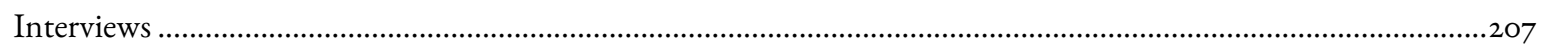

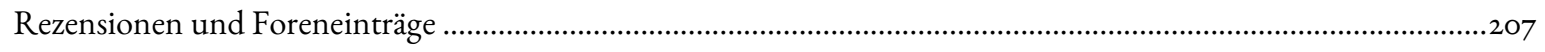

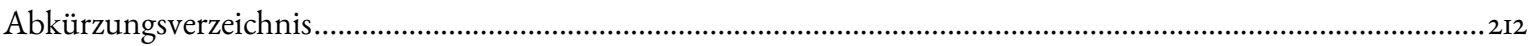

\section{Abbildungs- und Tabellenverzeichnis}

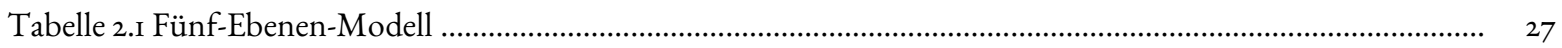

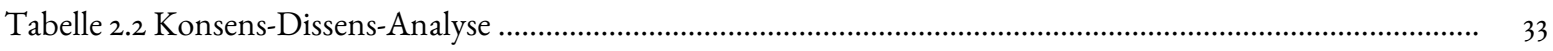

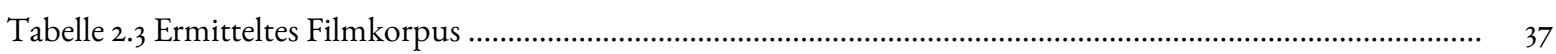

Abbildung 2.I Konsens/Dissens, ob ein Film neuseeländisch ist .......................................................................

Abbildung 2.2 Konsens/Dissens - zeitlicher Verlauf ..................................................................................... 4I

Abbildung 2.3 Entwicklung Anzahl neuseeländische Filme, 1977-2008 …........................................................

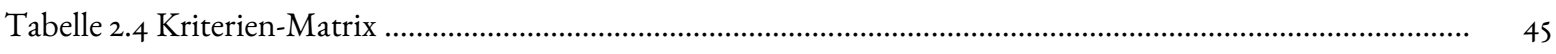

Abbildung 2.4 Entwicklung des Sektors „Produktionsfirma“ ........................................................................... 56

Abbildung 2.5 Förderanteil NZFC / Anteil neuseeländischer Filme am neuseeländischen Kinomarkt .................... 58

Abbildung 2.6 Entwicklung Bevölkerungsanteil Māori, I99I-20I2 _.................................................................... 6I

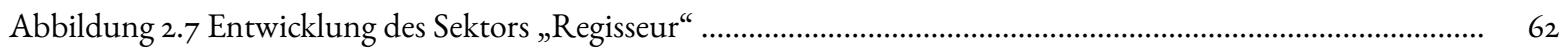

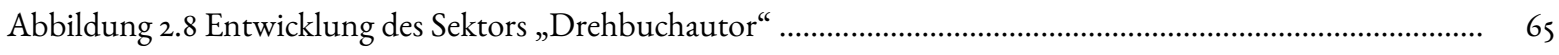

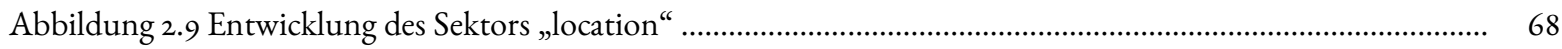

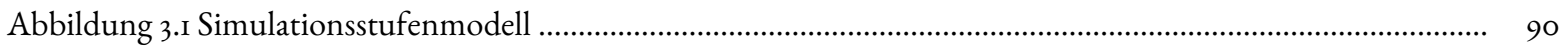

Abbildung 3.2 Delokalisierung und Entleerung von/Befüllung mit Landschaftsmarkern .................................... IO. IO2

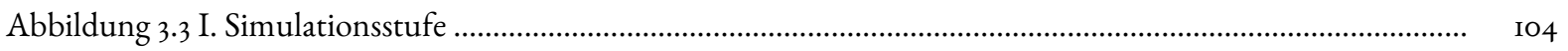

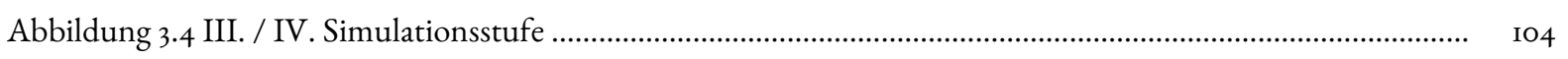

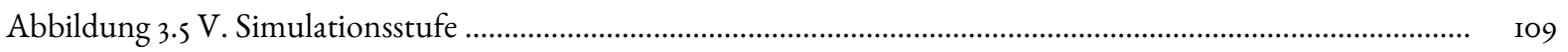

Abbildung 3.6 VI. Simulationsstufe mit realitätsbasiertem Handlungsort .............................................................. IIO

Abbildung 3.7 VII. Simulationsstufe mit fiktivem Handlungsort ......................................................................... IIO

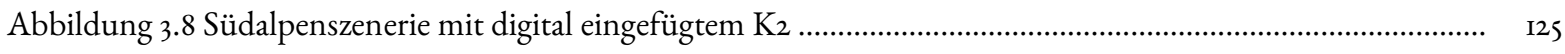

Abbildung 3.9 Angriff der Samurai, mit neuseeländischen Riesenfarnen ...............................................................

Abbildung 3.ro Google Streetview-Ausschnitt aus Raetihi [links], Filmszene aus Without a Paddle ....................

Abbildung 3.II Showdown-Szene / in der Postproduction digital eingefügte neuseeländische location ................... $\quad{ }_{14} 8$

Abbildung 3.12 Endszene des Films Willow (Nelwyn-Tal) ............................................................................ I57

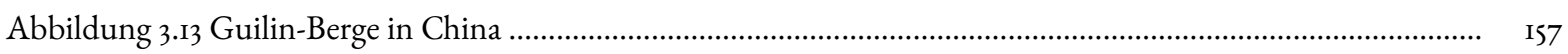

Abbildung 3.14 Vergleich I zwischen Filmszene aus Willow und neuseeländischer location .................................... I59

Abbildung 3.15 Vergleich 2 zwischen Filmszene aus Willow und neuseeländischer location ................................... I59

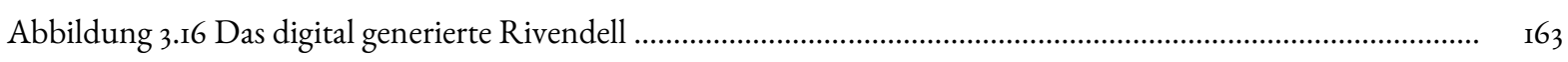

Abbildung 3.17 Argonath-Steinfiguren an Anduin-Flussmündung ….................................................................

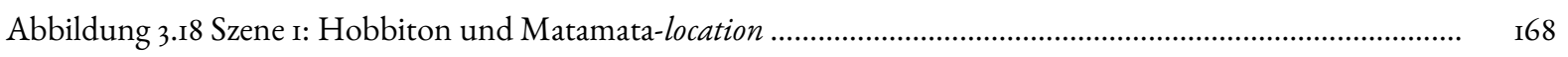

Abbildung 3.19 Szene 2: Weathertop / Port Waikato-location ............................................................................ I69 
Abbildung 3.20 Szene 3: Isengart / Glenorchy-location ..........................................................................................

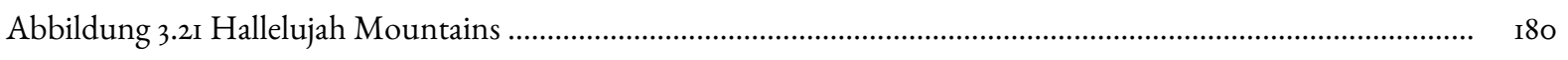

Abbildung 3.22 Karstgebirge des Zhangjiajie-Nationalparks .............................................................................. I85

Abbildung 3.23 Gigantische und rot gefärbte koru in Avatar / neuseeländische koru ........................................... I86 


\section{Einleitung}

In einer Welt voller Nationen ist es zur Selbstverständlichkeit geworden, in vielen Bereichen des Lebens in nationalen Bahnen zu denken: Es gibt „französische Küche“, „englische Fußballteams“ und „deutsche Autos“. Gerade in Zeiten intensivierter Globalisierung wird aber schnell deutlich, dass diese Kategorien alles andere als eindeutig sind und eigentlich hinterfragt werden müssten. ${ }^{\mathrm{I}}$ Dies gilt auch für Spielfilme, die gruppenweise bestimmten Ländern zugeordnet und dann als Vertreter eines „nationalen Kinos“ definiert werden. In vielen Filmlexika finden sich Einträge zu „französischem Kino“, „deutschem Kino“, „schwedischem Kino“ usf., auf der Internet Movie Database (IMDb.com), der weltweit größten Filmdatenbank, kann man Filme nicht nur nach Genre oder Jahr, sondern auch nach Ländern sortieren lassen, und auf Kino.de kann man sogar auf einer Weltkarte zuerst einen Kontinent und anschließend ein bestimmtes Land auswählen, um dann eine Auflistung sämtlicher Filme dieses Landes zu erhalten.

Hierbei betrifft die Zuordnung von Filmen nach Ländern die räumliche Ebene - und dies gleich in mehrfacher Hinsicht: Wenn man einen Film einem bestimmten Land zuordnet, dann aus dem Grund, dass der Film von einer dort ansässigen Filmcrew gedreht wurde, dass der Film sich inhaltlich auf dieses Land bezieht und/oder dass der Film dort gedreht wurde. Die Besonderheit dieser räumlichen Bezüge ist, dass Filme hierdurch oft auf etwas „Reales“ außerhalb ihrer eigenen filmischen, virtuellen Ebene verweisen, was wiederum Filmtourismus zur Folge haben kann. Der Filmtourismus kann sich dabei auf den Drehort und auf das vom Handlungsort „repräsentierte“ Land richten, wenn beispielsweise Braveheart ${ }^{2}$-Filmtouristen sowohl Schottland (Handlungsort) als auch Irland (Drehort) besuchen. Nationales Kino ist somit eine Kategorie, die auch nach ihren Bezügen zu symbolischen, aber auch „realen“, d. h. lebensweltlichen Räumen bzw. Geografien, definiert wird. Doch inwiefern kann man angesichts zunehmender globaler Verflechtungen und transnationaler Produktionsströme überhaupt noch vom „nationalen Kino“ sprechen und wie wäre dieses zu definieren?

Globalisierungsprozesse und die damit einhergehende sogenannte Digitale Revolution haben die Darstellungen filmischer Geografien tief greifend verändert. Zum einen ist es heute nichts

\footnotetext{
${ }^{\text {I }}$ So werden beispielsweise Automarken wie Mercedes-Benz, BMW und Porsche mit dem Gütelabel „Made in Germany“ versehen, obwohl die Fertigungsteile aus verschiedensten Ländern stammen und auch große Teile der Herstellung mittlerweile ins Ausland ausgelagert werden.

${ }^{2}$ Braveheart (1992) behandelt ein dramatisches Stück schottischer Zeitgeschichte, als der Nationalheld William Wallace (Mel Gibson) gegen die englische Willkürherrschaft aufbegehrte. Der Handlungsort des Films ist Schottland, gedreht wurde aber hauptsächlich in Irland.
} 
Ungewöhnliches mehr, wenn ein Film in einem Land gedreht wird, das keinerlei Bezug zum filmischen Handlungsort hat, wie beispielsweise der Dreh von Sieben Jabre in Tibet in den Anden. Zum anderen kommt es auch zu einer zunehmenden Digitalisierung von immer mehr Produktionsschritten filmischer Produktionen, so zum Beispiel die Erzeugung digital generierter „Schauspieler" wie die bekannte Gollum-Figur aus Lord of the Rings, aber auch - und das ist hier von besonderem Interesse - die Digitalisierung filmischer Drehorte bzw. locations ${ }^{3}$. Die Digitalisierung der location kann im Extremfall bedeuten, dass überhaupt keine location mehr zum Einsatz kommt, sondern allein auf digital generierte Landschaften und Räume zurückgegriffen wird, vor denen die Schauspieler dann agieren. Hinsichtlich des Zusammenhangs zwischen der Kategorie des nationales Kinos und der Kategorie des filmgeografischen Raums stellt sich somit folgende Frage: Bedeutet der zunehmende Verzicht auf locations in filmischen Produktionen etwa das Ende der location und letztlich auch das Ende des nationalen Kinos?

Diesen zentralen Fragen möchte sich die vorliegende Arbeit mittels kulturmaterialistischer und filmgeografischer Ansätze am Beispiel des neuseeländischen Kinos widmen. Das neuseeländische Kino ist ein anschauliches Analyseobjekt, wenn es darum geht, zum einen das Einwirken globaler Prozesse auf das problematische Konzept des „nationalen Kinos“ zu untersuchen, zum anderen die Beziehung zwischen location und Handlungsort, d. h zwischen „realer“ und fiktiver / fiktionalisierter Geografie zu untersuchen. Zum einen ist das neuseeländische Kino mit rund 200 zeitgenössischen Filmen noch überschaubar, zum anderen lassen sich anhand von transnationalen Koproduktionen wie Lord of the Rings, Last Samurai und Avatar, an denen die neuseeländische Filmindustrie entscheidend beteiligt war, die Globalisierungseinflüsse, die Digitalisierung und die filmtouristischen Folgen besonders anschaulich erörtern. Gerade ein Film wie Lord of the Rings, der Neuseeland größere Bekanntheit und intensiven Filmtourismus einbrachte, wirft hier interessante Fragen auf: Kann ein solcher Film überhaupt als „neuseeländischer“ Film eingestuft werden, wo er sich doch inhaltlich gar nicht mit Neuseeland auseinandersetzt, sondern mit der rein fiktiven Fantasywelt „Mittelerde“? Können neuseeländische Drehorte wirklich adäquat sein, um eine

\footnotetext{
${ }^{3}$ Im Folgenden soll der englische Begriff location anstelle des deutschen Begriffs „Drehort“ verwendet werden, da der englische Begriff eindeutiger auf Außenaufnahmen in freier Landschaft verweist, wohingegen der deutsche Begriff zweideutig ist und sowohl auf Außenaufnahmen als auch auf Aufnahmen in einem Filmstudio verweisen kann.

${ }^{4}$ Zwar betrachtet ein großer Teil der Neuseeländer den Film als eine Art Aushängeschild für ihr Land, als Zeichen einer typisch neuseeländischen kiwi ingenuity, d. h. Experimentierfreudigkeit und Improvisationstalent, und propagiert auch eine starke Verbindung zwischen dem realen Neuseeland und dem fiktiven „Mittelerde“. Andere jedoch, wie der Māori-Neuseeländer Alan Duff, der den Roman Once Were Warriors verfasste, sehen hingegen überhaupt keinen Zusammenhang zwischen Neuseeland und Lord of the Rings: „Was hat Peter Jacksons Hobbit-Film mit Neuseeland zu tun? Gar nichts. Es ist empörend, wie weit sowohl Landschaft und Fantasywelt als auch das Leben der Menschen und das der Kinobesucher auseinanderklaffen." Duff stellt in seinem kritischen Kommentar in der Süddeutschen Zeitung klar, dass er Lord of the Rings und The Hobbit nicht als neuseeländische Filme
} 
Fantasywelt darzustellen, die in keiner Beziehung zur Geografie Neuseelands steht? Was sahen die Zuschauer in Lord of the Rings an spezifisch „Neuseeländischem“, dass sie den Film mit Neuseeland assoziierten und daraufhin als Filmtouristen nach Neuseeland reisten?

Diese Fragen stellen sich nicht nur bei der Lord of the Rings-Trilogie, sondern für eine Vielzahl neuseeländischer Filme. Selbst bei Filmen wie The Piano und Whale Rider, die aufgrund ihres Inhalts als besonders „typische“ Beispiele des neuseeländischen Kinos angesehen werden, handelt es sich letztlich um transnationale Koproduktionen - im Falle von The Piano sogar ganz ohne Mitwirkung einer neuseeländischen Produktionsfirma. ${ }^{5}$ Hieran wird deutlich, dass gar nicht klar ersichtlich ist, welche Kriterien eigentlich in welcher Gewichtung herangezogen werden, um einen Film als „neuseeländisch“ zu definieren - und dass diese Definitionsfrage durch die zunehmende Globalisierung auch immer schwieriger zu beantworten ist. Mit diesen Problematiken wird sich das zweite Kapitel eingehend auseinandersetzen. Ziel ist hierbei, nicht nur eine Definition des nationalen Kinos zu erarbeiten, sondern am neuseeländischen Filmkorpus selbst zu untersuchen, inwiefern das neuseeländische Kino „denationalisiert“, d. h. durch Globalisierungsprozesse in seiner Kohärenz destabilisiert worden ist und ob es umgekehrt Resistenzen und „renationalisierende“ Gegenmaßnahmen gibt. Es soll also ein Gesamtblick auf das (zeitgenössische) neuseeländische Kino geworfen und ermittelt werden, ob es bestimmte Faktoren gibt, die bei der Einstufung eines Films als „neuseeländisch“ besonders ausschlaggebend sind.

Hierbei stellt sich das Problem, dass die Beurteilung eines Filmkorpus von rund 200 Spielfilmen kaum durch inhaltliche Analysen sämtlicher Filme bewerkstelligt werden kann. Ein „Gesamtblick“ auf zumindest eine Ebene kann allerdings erreicht werden, wenn diese Ebene auf empirischem Wege in ihrer Gänze erfasst werden kann. Die Ebene, die sich hierfür anbietet, ist die Produktionsebene der Filme, d. h. die Frage nach der Nationalität der Produktionsfirmen, der Regisseure, der Drehbuchautoren und der locations, ${ }^{6}$ da diese Attribute eindeutig bestimmbar sind. Das zweite Kapitel wird demnach eine kulturmaterialistische Analyse der Produktionsebene des neuseeländischen Kinos darstellen. Ein Vorteil dieses Fokus ist, dass hierdurch auch Filme berück-

begreift und kritisiert nicht nur Peter Jackson und die Hollywood-Industrie, die sich nicht um spezifisch neuseeländische Themen kümmere, sondern auch die Zuschauer dieser Fantasyfilme als einem von Eskapismusbedürfnissen getriebenen „Virtual-RealityMob“ (Duff, 20I2).

${ }^{5}$ Während es sich bei Whale Rider um eine deutsch-neuseeländische Produktion handelt, bei der unter anderem die Filmstiftung Nordrhein-Westfalen und die New Zealand Film Commission mitgewirkt haben, wurde The Piano ausschließlich von den nichtneuseeländischen Produktionsfirmen Ciby 2000 (Frankreich), Jan Chapman Productions, New South Wales Film \& Television Office und der Australian Film Commission finanziert.

${ }^{6}$ Zweifellos könnte auch nach der Nationalität weiterer Attribute der Produktionsebene wie beispielsweise dem Kameramann, dem Tontechniker usw. gefragt werden. Meines Ermessens sind die genannten Attribute aber die wichtigsten und ausreichend, um generalisierbare Aussagen treffen zu können. 
sichtigt werden können, die zwar inhaltlich keinen Bezug zu Neuseeland haben, aber gerade aufgrund ihres Produktionshintergrundes identitätsstiftendes Potenzial haben, wie beispielsweise die Lord of the Rings-Trilogie, die die „Qualitätsarbeit“ und Kreativität der neuseeländischen Filmindustrie herausstellen soll.

Hingegen werden inhaltsbasierte Erörterungen, also die Analyse von Stereotypen, Figuren, der Auseinandersetzung mit neuseeländischer (Post-)Kolonialgeschichte sowie idiosynkratische Filmstile und -ästhetiken nur einen Nebenfokus bilden. Zweifellos ist diese Einschränkung nicht gänzlich befriedigend, da Filme natürlich auch anhand ihrer Inhalte als „neuseeländisch“ definiert werden können. Um diese Problematik etwas zu entschärfen, werden im Anschluss an die Analyse der Produktionsebene zusätzliche Überlegungen zur Inhaltsebene neuseeländischer Filme angestellt werden. An dieser Stelle sei jedoch angemerkt, dass diese Überlegungen eine ähnlich umfassende und tiefer gehende Analyse wie die der Produktionsebene nicht zu ersetzen vermögen - hier besteht also noch Forschungsbedarf, um umfassendere Kenntnisse über das Konstrukt des nationalen Kinos zu erlangen.

Der Frage, was „nationales Kino“ theoretisch betrachtet genau ist, wird in den Filmwissenschaften erst in jüngerer Zeit kritisch nachgegangen. Während früher, häufig im Kontext der auteur-Theorie, „nationales Kino“ als stabile und kaum hinterfragbare „Common Sense“Kategorie erachtet wurde, haben seit den I980ern Forscher wie Stephen Crofts, John Hill und Andrew Higson die Kategorie des nationalen Kinos als widersprüchliche Kategorie problematisiert (Higson, 1989; Hill, 1992; Crofts, 1998; siehe auch: Hjort/MacKenzie, 2002). Higson, dessen primäres Interesse dem britischen Kino gilt, kommt in seinem Aufsatz „The Concept of a National Cinema" von 1989, einer der ersten generellen und kritischen Abhandlungen über nationales Kino (Crofts 1998: 386), zu dem Schluss, dass es keinen allgemeinen Konsens über den Diskurs um nationales Kino gebe („There is not a single universally accepted discourse of national cinema“ [Higson 1989: 52]) und dass zwischen vier verschiedenen Ansätzen, nationales Kino zu definieren, differenziert werden könne: einem ökonomischen Ansatz, einem textbasierten Ansatz, einem rezeptionsbasierten Ansatz und einem kritikbasierten Ansatz (52-53). Während er insbesondere den kritikbasierten Ansatz, bei dem selektiv nur diejenigen Filme als „repräsentativ“ für ein nationales Kino erachtet werden, die besonders prestigeträchtig erscheinen, als essenzialistisch kritisiert, betont er die Bedeutung des rezeptionsbasierten Ansatzes, wonach die nationale Zuschauerschaft durch ihren Konsum nationaler und ausländischer Filme ihre kulturelle Identität konstruiert (65). Dieser 
Ansatz ist wiederum von John Hill kritisiert worden, da der vorwiegende Konsum von Hollywoodfilmen in Großbritannien nach diesem Ansatz zu der paradox anmutenden Schlussfolgerung führen würde, dass Hollywoodfilme Teil des nationalen britischen Kinos seien (Hill 1992: I4). Die Kritik erscheint mir berechtigt, da es zwar durchaus sinnvoll ist, bei speziellen Fragestellungen die Ansichten der Zuschauer miteinzubeziehen, „nationales Kino“ aber auch im Falle Neuseelands vor allem top down, beispielsweise von der New Zealand Film Commission (NZFC), konstruiert wird, ohne hierbei allzu viel Wert auf den tatsächlichen Konsum dieser als „neuseeländisch“ erachteten Filme durch neuseeländische Zuschauer zu legen. ${ }^{7}$

Konkret mit der Frage nach einem nationalen neuseeländischen Kino beschäftigen sich vor allem die New Zealand Film Commission, Conrich/Murrays Contemporary New Zealand Cinema: From New Wave to Blockbuster, Martin/Edwards' New Zealand Film: 19I2-19g6, und Mayer/ Beatties The Cinema of Australia and New Zealand. Während die New Zealand Film Commission, Conrich/Murray und Martin/Edwards Filmlisten bzw. Filmkataloge über sämtliche von ihnen als „,neuseeländisch“ erachtete Filme liefern und dabei vorwiegend sowohl nach ökonomischen als auch inhaltlichen Kriterien vorgehen, also den ökonomischen mit dem textbasierten Ansatz Higsons kombinieren, folgt Mayer (implizit) dem von Higson als essenzialistisch kritisierten kritikbasierten Ansatz. Zwar problematisiert auch Mayer kurz die Kategorie des nationalen neuseeländischen Kinos, die anschließend in den Beiträgen behandelten Filme wie Vigil, The Piano, Once Were Warriors und Lord of the Rings sind aber ausnahmslos Filme, die kritisch und/oder kommerziell am erfolgreichsten gewesen sind. Während Higson vor allem im Hinblick auf diesen kritikbasierten Ansatz bemängelt, dass nationales Kino meist als präskriptive und nicht als deskriptive Kategorie behandelt wird, setzen letztlich außer dem rezeptionsbasierten Ansatz alle von ihm beschriebenen Ansätze eine normative, präskriptive Herangehensweise voraus. Auch bei den nach ökonomischen und textuellen Kriterien konzipierten Auflistungen neuseeländischer Filme in Conrich/Murray, Martin und der NZFC musste entschieden werden, ob beispielsweise ein Film mit neuseeländischer location und neuseeländischem Regisseur, aber ausländischem Drehbuchautor, ausländischer Produktionsfirma und nicht-neuseeländischem Handlungsort als „neuseeländisch“ oder bereits als „ausländisch“ einzustufen sei.

\footnotetext{
${ }^{7}$ So ist beispielsweise Barry Barclays Film Ngati (1987) zwar von Kritikern gelobt worden, aber finanziell aufgrund der sehr geringen Zuschauerschaft nicht erfolgreich gewesen - und dennoch sowohl von der NZFC als auch anderen als „neuseeländischer“ Film mit einem „specific New Zealand content“ eingestuft worden.
} 
Ziel des zweiten Kapitels wird es sein, diesen Forderungen nach mehr Deskriptivität und weniger Präskriptivität bei der Analyse der Kategorie des nationalen Kinos entgegenzukommen. Wenn lediglich einzelne Filme des neuseeländischen Kinos als „repräsentativ“ dargestellt werden, ist hiermit immer die Notwendigkeit einer subjektiven Selektion verbunden, bei der viele Filme unberücksichtigt bleiben - ein Ansatz der zwangsläufig präskriptiv ist. Gerade um derartiger Präskriptivität möglichst entgegenzuwirken, wird stattdessen ein empirischer Ansatz gewählt und hierbei bereits bestehende Konstruktionen des nationalen neuseeländischen Kinos - in erster Linie diejenigen der oben genannten Filmlisten von Conrich/Murray, Martin/Edwards, der New Zealand Film Commission und zusätzlich der Internet Movie Database - miteinander verglichen werden. Anschließend wird eruiert, bis zu welchem Grad über einzelne Produktionsfaktoren ein Dissens oder Konsens herrscht, wenn es um die Einstufung einzelner Filme als „neuseeländisch“ geht und wie sich diese Produktionsfaktoren angesichts zunehmender Globalisierung verändert haben. Im letzten Schritt wird schließlich analysiert werden, inwiefern sich die einzelnen Produktionsfaktoren unter dem Einfluss der Globalisierung verändert haben, d.h. de- oder renationalisiert worden sind.

Während im zweiten Kapitel somit der Frage nach der De- und Renationalisierung des neuseeländischen Kinos auf der Produktionsebene nachgegangen wird, wird das dritte Kapitel, das den Hauptteil dieser Studie bilden wird, die Bedeutung der location als besonderem Produktionsfaktor in ihrer funktionalen Vielfalt beleuchten. Im Vergleich zu den anderen im zweiten Kapitel zu analysierenden Produktionsfaktoren wie Produktionsfirma, Regisseur oder Drehbuchautor, spielt die neuseeländische location als eine im Hintergrund wirkende ästhetische Instanz eine herausragende Funktion bei der Definition des neuseeländischen Kinos: Sie dient als Grundlage zur Konstruktion sowohl neuseeländischer als auch nicht-neuseeländischer Handlungsorte, ist zentral für die Selbstvermarktung Neuseelands als Produktionsstandort für große transnationale Filmproduktionen, spielt eine wichtige Rolle bei der Zuschauerrezeption und hat eine erhebliche Bedeutung für die Generierung von Filmtourismus.

Diese filmgeografischen Aspekte verweisen auf das übergeordnete Spannungsfeld zwischen Fiktion und Realität. Während jedoch quasi seit den Anfängen der Filmwissenschaft auf dieses Wechselspiel zwischen Fiktion und Realität auf narrativischer Ebene der Filme eingegangen wurde, werden filmgeografische Aspekte in dieser Hinsicht erst seit jüngerer Zeit behandelt. Wie Piatti anführt, wurde die Kategorie des (geografischen) „Raums“ in den deutschsprachigen Geisteswis- 
senschaften aufgrund ihres Missbrauchs im Rahmen der nationalsozialistischen Ideologie lange Zeit nur sehr stiefmütterlich behandelt (2008: 80). In jüngerer Zeit sieht sie allerdings in der literaturgeografischen Forschung eine „selbstbewusste Rückkehr“ zu „Fragen des Raumes“(86). Eine ähnliche Rückkehr zu raumphilosophischen Fragen will die Filmgeografie für die Filmwissenschaften leisten. Die Disziplin der Filmgeografie ist ein relativ junger interdisziplinärer Ansatz, der sich unter anderem aus dem spatial turn in den Cultural Studies ergeben hat, wie Lukinbeal anführt: „With the 'spatial turn' in cultural studies the focus on visual media and visual culture has been modified to incorporate the role of space and place in representational theory, identity formation and the role of Hollywood in globalization“" (2004: 248). Lukinbeal definiert Filmgeografie als die Analyse der Interaktion sozialer Prozesse mit dem Raum und merkt an, dass die filmische Vermittlung Räume mit neuen Bedeutungen aufladen kann: „Cinematic Geography focuses on how social and cultural meanings are intertwined with space, place, scale and narrative. [...] Traditional concepts like space, place, distance and scale take on new meanings when we engage film and television“ (248-249). Er vertritt dabei eine konstruktivistische Sicht, die er sowohl auf den Gegenstand filmischer Darstellung, d. h. „Landschaft“ und „Natur“, als auch auf das darstellende Medium selbst, d. h. den Film, anwendet: „'New’ cultural geographers argued that concepts like 'nature’ and 'landscape' are sophisticated social constructions, not unlike film, media, books and painting. [...] Film is therefore a cultural construct that is intertextually related to other systems of cultural production and reproduction such as landscape“ (249).

Zimmermann verweist in diesem Zusammenhang darauf, dass Filme neue Landschaften konstruieren: „Der Spielfilm erschafft die Landschaft, da das Medium aufgrund der Präsentationstechnik einen subjektiv gerichteten Blick aus einem bestimmten Betrachtungswinkel, d. h. nur einen genau definierten Ausschnitt thematisiert. Filme kreieren Landschaften, die es ohne Spielfilm in dieser Art nicht gäbe“ (200I: 229). Das bedeutet, dass sich Filmlandschaft sowie filmisch dargestellte „Natur“ durch ihre doppelte Konstruiertheit von der physisch vorhandenen Landschaft und Natur unterscheiden: Während Landschaft und Natur auch eine nicht-konstruierte materielle Realität besitzen (und daher als Ressource verbraucht werden können), ist Filmlandschaft ein virtuelles, auf die audiovisuelle, zweidimensionale Fläche der Filmleinwand projiziertes Konstrukt, das durch die festgelegte Perspektive der Filmemacher vermittelt wird.

Während in den meisten filmgeografischen Arbeiten allein auf die inhaltliche und ästhetische Funktion des Handlungsortes bzw. Settings fokussiert wird, soll im Rahmen dieser Arbeit auf 
die noch wenig erforschte Beziehung zwischen Handlungsort und location eingegangen werden. Im Rahmen der filmgeografischen Analyse wird hier auf einen kulturmaterialistischen Raumbegriff abgestellt: Es soll bei den Analysen weniger um eine symbolische, narrativische Raumkonstruktion gehen, sondern in erster Linie um die Beziehung zwischen der location als lebensweltlichem Raum und dem Handlungsort als filmisch konstruiertem Raum. Im Gegensatz zu einem radikalkonstruktivistischen, poststrukturalistischen Raumbegriff kann mittels eines die materiellen Aspekte des Raumes berücksichtigenden Raumbegriffs die Beziehung zwischen virtuellem filmischem Raum und lebensweltlichem „realen“ Raum auf sinnvolle Weise beschrieben und analysiert werden und trägt damit auch den materialistischen Ursprüngen des Feldes der Geografie Rechnung.

Den Gedankengang, den das dritte Kapitel nachzeichnen will, ist Folgender: Wenn Filmemacher aufgrund transnationaler Praktiken ein Auseinanderdriften der Geografien von location und Handlungsort in ihren Filmen in Kauf nehmen, führt dies bei Zuschauern zur Infragestellung der filmgeografischen „Glaubwürdigkeit“ dieser Filme - und wenn dem so ist, welche Auswirkungen hat dies wiederum auf das filmtouristische Potenzial dieser Filme? Ziel ist es hierbei, ein übergeordnetes „Simulationsstufenmodell“ zu erarbeiten, mit denen sich die genannten Aspekte und Fragestellungen systematisch beantworten lassen.

\section{Auseinanderdriften der Geografien von location und Handlungsort}

Neuseeland zeigt seit Längerem ein großes Interesse daran, durch Steuervergünstigungen und Förderungsprogramme wie dem Large Budget Screen Production Grant gezielt runaways, d. h. Hollywoodproduktionen, die außerhalb der USA produziert und gedreht werden, anzulocken. Durch Rückgriff auf die mittlerweile weltweit bekannte neuseeländische Landschaft als wirtschaftlicher und kultureller Ressource in Filmproduktionen erhofft sich Neuseeland eine wirtschaftliche Stärkung ihrer vergleichsweise kleinen lokalen Filmindustrie und nicht zuletzt auch die Erzeugung von Filmtourismus.

Da Hollywood generell bisher kaum Interesse daran zeigte, Filme mit neuseeländischem Handlungsort und neuseeländischer story zu drehen, hat diese transnationale Produktionspraxis zu einem Phänomen geführt, das im Folgenden als „Delokalisierung“ bezeichnet werden soll: dem Einsatz der neuseeländischen location außerhalb ihres kulturellen und historischen Kontextes in immer mehr Filmen. Während bei Filmen wie Whale Rider und The Piano sowohl location als auch Handlungsort neuseeländisch und somit in geografischer Hinsicht gewissermaßen „de- 
ckungsgleich“ sind, ist dies bei den hier vorrangig zu betrachtenden runaways, die in Neuseeland gedreht werden, nicht mehr der Fall. So wird in Filmen wie Last Samurai die neuseeländische location eingesetzt, um einen nicht-neuseeländischen - in diesem Falle japanischen - Handlungsort zu konstruieren, einem Handlungsort also, der in keinerlei geografischer oder kultureller Beziehung zu Neuseeland steht. Zwar ist Landschaft und Raum einer location nicht intrinsisch „national“, durch Zuschreibungsprozesse werden diese aber „politisiert“, d. h. bestimmte Landschaften und Räume bestimmten Nationalstaaten zugeschrieben. Sowohl die Neuseeländer selbst als auch die Touristen definieren die neuseeländische Landschaft aufgrund bestimmter Charakteristika (z. B. endemische Flora und Fauna, charakteristische Geomorphologien) als „neuseeländisch“. Wenn nun aber in runaways gerade diese Charakteristika weitgehend entfernt werden, um die filmgeografische Inkongruenz zwischen location und Handlungsort weniger offensichtlich zu machen, wird die neuseeländische location hiermit ihrer „Neuseeländischkeit“ in großen Teilen beraubt und stattdessen als transnationale bzw. „anationale“ Projektionsfläche eingesetzt.

Nun hat Neuseeland nicht nur durch seine locations Bekanntheit erlangt, sondern auch durch seine Postproduction-Anlagen. Insbesondere die von Peter Jackson begründeten WETA digital-Studios, die sich seit der Lord of the Rings-Produktion zu einer „state of the art“-Einrichtung entwickelt haben, kann nach Ansicht vieler Filmemacher mit etablierten Hollywood-Postproduction-Einrichtungen wie Industrial Light and Magic ${ }^{8}$ konkurrieren. In den WETA-Studios werden längst nicht mehr nur digitale Charaktere wie beispielsweise die bekannte Gollum-Figur in Lord of the Rings oder der Riesenaffe in King Kong erzeugt, sondern immer öfter auch ganze Landschaften und Welten digital generiert, z. B. für Adventures of Tintin, King Kong und Avatar. Auch dieser Faktor lockt runaways nach Neuseeland, um von dem dortigen technischen Know How bei der Erzeugung digitaler Charaktere und Welten zu profitieren - einer Praxis, die sich gerade bei der Produktion von Fantasy- und Science Fiction-Filmen immer größerer Beliebtheit erfreut.

Hierdurch kommt es zu einem Prozess, der nicht mehr nur eine Delokalisierung der neuseeländischen location darstellt, sondern etwas viel Schwerwiegenderes: Der teilweisen oder auch völligen Ersetzung neuseeländischer locations durch CGI'-Landschaften und -Räume im filmischen Produktionsprozess, was im Folgenden als „Entlokalisierung“ bezeichnet werden soll. Das unmittelbare Konkurrenzverhältnis zwischen digitalen CGI-Geografien und neuseeländischen locations

\footnotetext{
${ }^{8}$ Industrial Light and Magic wurde von George Lucas gegründet und ist vor allem durch die Star Wars-Filmreihe bekannt geworden.

${ }^{9}$ CGI ist eine Abkürzung für computer generated imagery und verweist auf die unentbehrliche Rolle moderner Computer in der Erzeugung digitaler Bilder.
} 
lässt sich beispielsweise aufzeigen im Vergleich von Peter Jacksons Werken Lord of the Rings, The Hobbit und King Kong, die eine stete Verlagerung des Verhältnisses zwischen CGI und location zuungunsten der location aufzeigen: Während in Lord of the Rings noch eine Vielzahl neuseeländischer locations zum Einsatz kam, haben sich diese aufgrund des verstärkten Einsatzes von CGI in der Hobbit-Trilogie deutlich verringert, und bei King Kong schließlich werden neuseeländische locations praktisch überhaupt nicht mehr eingesetzt, sondern sämtliche Landschaften digital generiert - eine Entwicklung, die im dritten Kapitel eingehend erörtert wird.

De- und Entlokalisierung beschreiben demnach ähnlich wie die Denationalisierung Prozesse, die das neuseeländische Kino destabilisieren, nur dass im Falle der De- und Entlokalisierung speziell die filmgeografische Ebene der Filme betroffen ist. Eine Eigenart der Filmgeografie, die sie beispielsweise von der Literaturgeografie unterscheidet, ist die grundsätzliche Differenzierung zwischen zwei geografischen Ebenen im Medium Film: Dem virtuellen Handlungsort und der location eines Films, die die materielle räumliche Basis des Films bildet und in dieser Form auch keine Entsprechung in der Literaturgeografie findet. Hierbei gehört der Handlungsort zwar ganz offensichtlich zum Filmtext, aber auch die location, die der Produktionsebene eines Films zugeordnet werden kann, „ragt“ in den Filmtext hinein, da sie als location im Film sichtbar bleibt und in ästhetischer und funktionaler Hinsicht mit dem Handlungsort interagiert bzw. verwoben ist. Hierbei stellen De- und Entlokalisierung dieses filmgeografische Grundgerüst von Filmen infrage, wobei die Entlokalisierung letztendlich eine Steigerung der Delokalisierung darstellt: Während bei der Delokalisierung die neuseeländische location als Basis filmgeografischer Konstruktionen zwar verfremdet wird, in ihrer Materialität aber unangetastet bleibt, destabilisiert die Entlokalisierung das Verhältnis zwischen materieller, „realer“ location und virtuellem Handlungsort an sich. Es verschwindet also das „Reale“, der lebensweltliche geografische Raum, zunehmend aus der Produktion filmischer Handlungsorte und wird durch reine Digitalität ersetzt.

Das Verschwinden des „Realen“ angesichts der Omnipräsenz von Simulakren ist ein wichtiges Thema in Baudrillards Abhandlungen über Simulation und Hyperrealität. Er konstatiert eine Abfolge verschiedener distinktiver Stadien, wobei das letzte Stadium die Loslösung jeglicher Bezüge zu einer lebensweltlichen Realität und damit eine Art „Tod des Realen“ beschreibt (1981: 170). Baudrillards postmoderne Simulationstheorie eignet sich sehr gut zur Beschreibung der bisher erörterten Phänomene der De- und Entlokalisierung der neuseeländischen location. Analog zu Baudrillards Differenzierung in verschiedene Stadien der Loslösung der Simulation vom Realen, kann hier 
differenziert werden zwischen Filmen, in denen die neuseeländische location in ihrer Neuseeländischkeit verschleiert wird und Filmen, in denen die neuseeländische location an sich ersetzt wird und damit das Reale durch pure Simulation verdrängt wird.

\section{Authentizitätserwartungen der Zuschauer und Authentisierungsstrategien der Filmemacher}

Diese sukzessive Destabilisierung und Derealisierung des filmgeografischen Gefüges wirft wichtige Fragen bezüglich der Zuschauerrezeption auf: Wie gehen Zuschauer mit den Phänomenen der De- und Entlokalisierung um? Beeinträchtigen diese für Zuschauer die „Authentizität“ bzw. „Glaubwürdigkeit“ der filmgeografischen Konstruktionen? Und wie versuchen Filmemacher, die Zuschauer dazu zu bewegen, trotz De- und Entlokalisierung die filmgeografischen Konstruktionen dennoch zu akzeptieren?

Spielfilmen wird zwar in erster Linie Unterhaltungswert zugeschrieben, dies bedeutet aber nicht, dass sie nicht auch (geografischen) Informationswert besitzen. Eine vollständige Filmwelt besteht nicht nur aus den Charakteren und deren Handlungen; das filmische Geschehen muss für den Zuschauer auch lokalisierbar sein, d.h. es gehört immer auch eine Geografie dazu, vor dessen Hintergrund sich das filmische Geschehen abspielt. Die Geografie und die Landschaft in Filmen ist hierbei keineswegs immer nur „Kulisse“, sondern kann wichtige Funktionen neben der allgemeinen Lokalisierungsfunktion haben, z. B. die Situation der Charaktere und deren Innenleben auf symbolische Weise externalisieren und dadurch sichtbarer machen oder z. B. als aktive Entität auftreten, gegen die sich die Charaktere behaupten müssen (z. B. in Bergfilmen). Zimmermann führt an, dass das Betrachten insbesondere landschaftsbetonter Filme für den Zuschauer auch eine Art Wissensquelle über die Geografie sowie den Charakter noch nie besuchter Länder oder Gebiete darstellt (2007: 25). Fühlen sich Zuschauer also um diesen geografischen Informationswert betrogen und fehlgeleitet, wenn beispielsweise im Film Water Horse: Legend of the Deep das filmisch dargestellte Schottland nicht mittels schottischer, sondern neuseeländischer locations gedreht wurde? Bei der Betrachtung dieser rezeptionsästhetischen Problematiken soll keineswegs unterstellt werden, dass Zuschauer filmische und reale Geografie gleichsetzen würden, es geht vielmehr darum, dass filmgeografische Inkongruenz dazu führen kann, dass Zuschauer diese Konstruktionen nicht mehr als „authentisch“ empfinden und sich dadurch in ihrer Immersion in das filmische Geschehen beeinträchtigt fühlen. 
Während „Authentizität“ früher mitunter als etwas Objektives erachtet wurde, hat sich heute in der kulturwissenschaftlichen Forschung die Sichtweise durchgesetzt, dass Authentizität subjektiv und konstruiert ist. Das Thema „Authentizität“ ist nach Daur seit der Diskussion um die Postmoderne und damit assoziierten Phänomenen wie Wiederholung, Kopien und „Fakes“ aus dem kulturwissenschaftlichen Diskurs fast verschwunden und erst in jüngster Zeit wieder populär geworden (2013: 7), wie die Sammelbände Renaissance der Authentizität? Über die neue Sebnsucht nach dem Ursprünglichen (Rössner/Uhl, 2014), Authentizität und Wiederholung (Daur, 2013) und The Paradox of Authenticity in a Globalized World (Cobb, 20I4) demonstrieren - diesmal aber in „neuem Gewand“ als etwas, das nicht zwangsläufig nicht-inszeniert sein muss, sondern durchaus Inszenierung und sogar Wiederholung beinhalten kann. In meiner Definition von Authentizität werde ich mich vorrangig auf Hattendorfs Konzeption von Authentizität als einem „Vertragsschluss“ zwischen Filmemachern und Zuschauern stützen (1999: 76-77), wobei ich nicht auf eine dokumentarische Konzeption von Authentizität, d. h. der Frage, ob filmischer „Schein“ und „Sein“ übereinstimmen, abstelle, sondern vielmehr danach frage, ob filmische Inszenierungen geografischer Räume glaubwürdig im Sinne einer possible world (Laure-Ryan, 199I) sein können. Es wird also zu untersuchen sein, welche Authentisierungsstrategien Filmemacher einsetzen, um den Zuschauer in seinen Authentizitätserwartungen zufriedenzustellen und ob diese Erwartungen dann auch tatsächlich erfüllt werden oder nicht.

\section{Filmtouristisches Potenzial von runaways}

Die Erörterung der Zuschauerrezeption wirft wiederum Fragen bezüglich des filmtouristischen Potenzials von in Neuseeland produzierten runaways auf. Wenn Zuschauer durch die filmgeografischen Inkongruenzen in runaways in ihren filmgeografischen Erwartungen enttäuscht und fehlgeleitet werden können, dann stellt sich letztlich auch die Frage, inwiefern dies das filmtouristische Potenzial solcher Filme beeinflusst. Während der Film The Piano, der noch keine runaway darstellte, in den I99oern zum ersten Mal signifikanten Filmtourismus nach Neuseeland auslöste, erzeugten seit 2002 sowohl Whale Rider als auch die runaways Lord of the Rings und The Hobbit Filmtourismus nach Neuseeland, wobei der durch die genannten runaways generierte Filmtourismus erheblich größer war als der durch Whale Rider erzeugte. Bei Filmen wie The Piano und Whale Rider sehen Zuschauer aufgrund der Übereinstimmung von location und Handlungsort (beides Neuseeland) einen unzweifelhaften geografischen Bezug zu Neuseeland und empfinden die 
Darstellung dieser filmischen Handlungsorte als „authentisch“. Daher verwundert es wenig, dass ein Teil dieser Zuschauerschaft, der ein starkes Interesse und womöglich sogar eine „Sehnsucht“ nach diesen Handlungsorten empfand, die entsprechenden locations in Neuseeland als Filmtouristen aufsuchte, um den Handlungsorten „gefühlsmäßig“ näherzukommen. Da bei diesen Filmen die Frage nach der filmgeografischen Authentizität von den Zuschauern als unproblematisch erachtet wird oder auch gar nicht erst aufkommt, werden diese Filme bei der Analyse einen Nebenfokus bilden - allerdings werden auch bei diesen Filmen Überlegungen zu deren möglicher De- und Entlokalisierung angestellt werden, da seit Lord of the Rings auch bei vorwiegend lokal produzierten Filmen wie Perfect Creature vermehrt CGI-Elemente eingesetzt werden.

Bei den näher zu analysierenden runaways hingegen entstehen durch das Auseinanderdriften der filmgeografischen Ebenen interessante geografische Mehrfachbezüge: Wenn Zuschauer beispielsweise ein filmtouristisches Interesse an weiter oben genanntem Film Water Horse: Legend of the Deep entwickeln, reisen sie dann zur location nach Neuseeland oder zum vom Handlungsort repräsentierten „realen“ Schottland? Weshalb konnte ein Film wie Lord of the Rings derart starkes filmtouristisches Interesse an Neuseeland erzeugen, wo doch die Zuschauer beim Betrachten des Film kaum die CGI-Landschaften von den eingesetzten neuseeländischen locations unterscheiden konnten? Und schließlich: Besitzen Filme wie King Kong und Avatar, die ohne reale locations auskommen und zudem rein fiktive Handlungsorte darstellen, überhaupt noch filmtouristisches Potenzial, wo sie doch Beispiele reiner (filmgeografischer) Selbstreferenzialität darzustellen scheinen?

Diese folgenschweren Implikationen für den neuseeländischen Filmtourismus sind bisher noch nicht eingehend behandelt worden, auch wenn es mittlerweile einige Studien wie beispielsweise Beetons Film-Induced Tourism (2005), Roeschs The Experience of Film Location Tourists (2009), Carls Cultural Representation of New Zealand's Landscapes in the Films of The Lord of the Rings and its Implications for Tourism (2004) und Leottas Touring the Screen: Tourism and New Zealand Film Geographies (20II) gibt. Während in diesen Arbeiten durchaus der Einsatz digitaler Effekte erwähnt wird, wird der zunehmenden Virtualisierung der locations und deren Folgen nur wenig Beachtung geschenkt, wobei Roesch zumindest auf die Unzufriedenheit vieler Filmtouristen eingeht, die aufgrund der weitreichenden digitalen Landschaftsmanipulationen in Lord of the Rings häufig kaum noch etwas vom filmisch konstruierten „Mittelerde“ in den locations wiedererkennen konnten (I52). 


\section{Simulationsstufenmodell}

Um die zu analysierenden runaways in verschiedene Handlungsort/location-Konstellationen bzw. Filmtypen einordnen zu können, an die die Zuschauer unterschiedliche Authentizitätserwartungen stellen und deren filmtouristische Implikationen sich mitunter deutlich unterscheiden, werde ich in meiner Analyse einen semiotischen Ansatz zu Hilfe nehmen. Hierbei werde ich jedoch nicht auf die - auf den ersten Blick naheliegende - Filmsemiotik bzw. Filmsemiologie zurückgreifen, wie sie vor allem von Christian Metz in Semiologie des Films (1972) und Sprache und Film (1973) konzipiert worden ist, da hierbei unter Zuhilfenahme psychoanalytischer Theorien auf die narrativische Ebene fokussiert wird, die für mich interessante formalästhetische, filmgeografische Ebene jedoch ignoriert wird. Vielmehr wird sich meine Arbeit auf bildsemiotische Ansätze stützen, ausgehend von dem von Ogden und Richards konzipierten semiotischen Dreieck (200I: 30) und weiteren Ausführungen von Charles Sanders Peirce, insbesondere seiner Differenzierung zwischen Ikon, Index und Symbol (1876: 56). Mittels dieser bildsemiotischen Ansätze lässt sich die Beziehung zwischen Handlungsort, Zuschauerimagination und location sehr gut darstellen, wie folgende Abbildung zeigt:
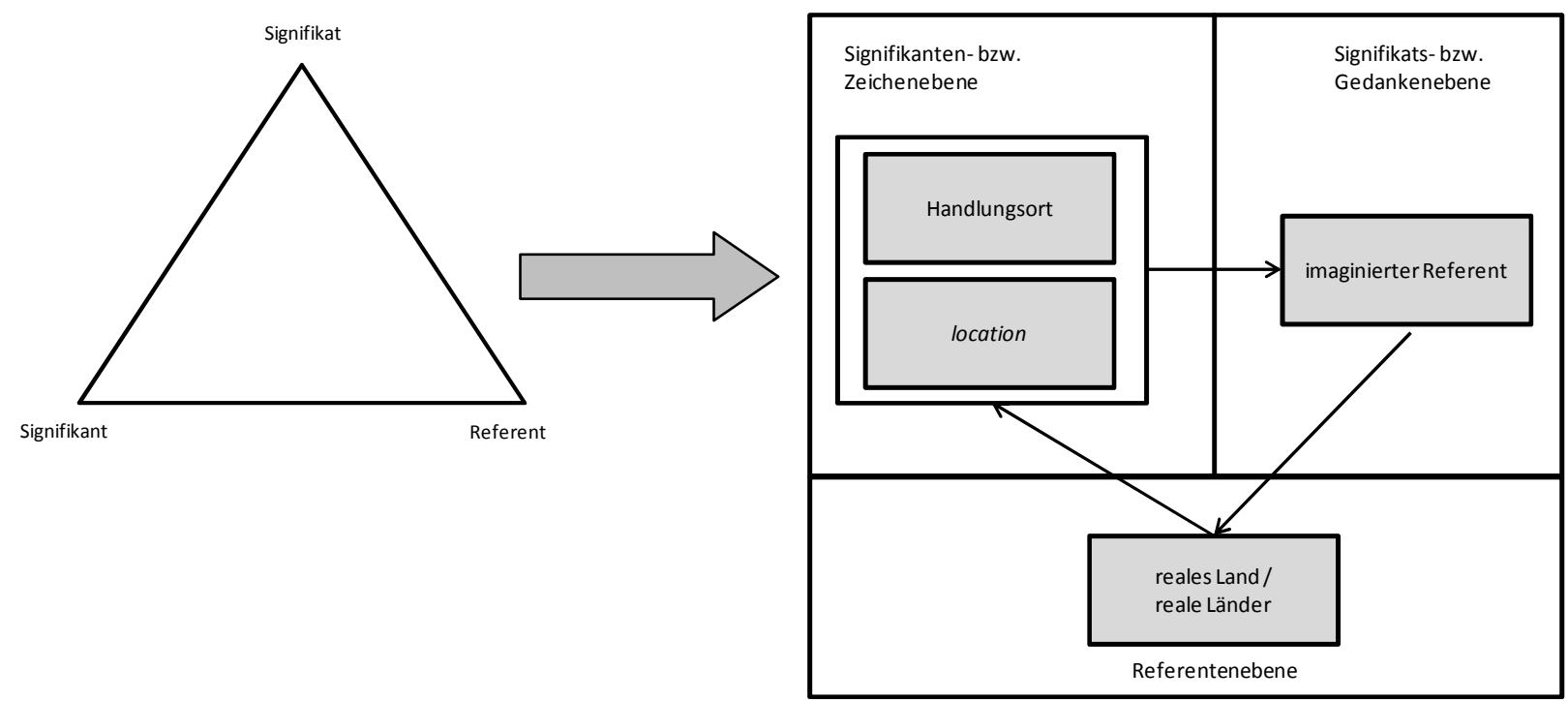

Die Abbildung zeigt die Übertragung der von Ogden/Richards erarbeiteten allgemeinen semiotischen Dreiecksbeziehung (linke Abbildung) in einen spezifisch filmgeografischen Kontext. Die rechte Abbildung stellt jedoch nur eine vereinfachte, komprimierte Darstellung der vielfältigen und teilweise sehr komplexen semiotischen Beziehungen zwischen Handlungsort, Zuschauerimagination und location dar. Es gibt beträchtliche Unterschiede zwischen den filmgeografisch- 
semiotischen Beziehungen eines Films wie Last Samurai und denen eines Films wie Avatar, die sich wiederum auf die Zuschauerrezeption und den Filmtourismus auswirken. Um diesen Unterschieden gerecht zu werden, werden die zu analysierenden runaways in einem übergeordneten siebenstufigen Simulationsstufenmodell kategorisiert. Ausgehend von Baudrillards Theorien einer sukzessiven Verzerrung und Verringerung der Referenzialität von Zeichen (1981: 170) stellt die erste Simulationsstufe mit Filmen wie Whale Rider die eindeutigsten filmgeografischen Bezüge her, wohingegen die siebte Simulationsstufe mit Filmen wie Avatar den extremen Gegenpol mit einem nahezu vollständigen Wegfall der Referentenebene darstellt. Die aufsteigende Folge der Simulationsstufen soll somit auch die Zuspitzung der filmgeografischen Problematiken versinnbildlichen, bei denen die grundlegenden Kategorien „Nation“ und „Raum“ immer weiter auseinanderdriften. Das Simulationsstufenmodell wird als strukturelle Grundlage für die anschließenden filmgeografischen Überlegungen und Filmanalysen dienen.

Die vorliegende Arbeit will somit die durch Globalisierung und Digitalisierung hervorgerufenen Prozesse, die das neuseeländische Kino nicht nur in seiner Konzeption als nationales Kino destabilisieren, sondern auch in seinen filmgeografischen Ausprägungen, die gerade bei dem an landschaftsfokussierten Filmen reichen neuseeländischen Kino eine überaus wichtige Rolle spielen, mittels kulturmaterialistischer, filmgeografischer und bildsemiotischer Ansätze näher beleuchten und Antworten auf die im Titel formulierten Fragen nach dem Ende des neuseeländischen Kinos und der neuseeländischen location finden. Denn in Zeiten zunehmender transnationaler Produktionsströme kann letztlich auch ein Land wie Neuseeland, dass sich mit abwechslungsreicher Landschaft, einzigartiger Flora und Fauna sowie einem clean $\mathcal{E}$ green-Image ${ }^{\mathrm{Io}}$ rühmt, keine unangefochtene Monopolstellung als location für runaways beanspruchen. Dies illustriert auch die sogenannte Hobbit-Krise, die im dritten Kapitel näher erörtert wird, wo sich in verdichteter Weise zeigte, wie viel Aufruhr - in positiver wie in negativer Hinsicht - die transnationalen Produktionsströme in einem „kleinen“ Land wie Neuseeland erzeugen konnten.

\footnotetext{
${ }^{\text {Io }}$ Mit dem clean \& green-Image und der „I00\% pure“-Werbekampagne vermarktet sich Neuseeland als Ioo\%ig intaktes Ökosystem - ein Ideal, das heute in Zeiten globaler Umweltschäden vermutlich in keiner Region der Erde mehr erreichbar ist. Dass auch Neuseeland von Umweltverschmutzungsproblematiken geplagt wird, illustriert die Debatte um den neuseeländischen Ökologieund Zoologie-Professor Dr. Joy, dessen Kritik an der neuseeländischen Umweltpolitik bei vielen Neuseeländern die Befürchtung ausgelöst hat, dass ein infragegestelltes clean $\mathfrak{\sigma}$ green-Image nachteilige Auswirkungen auf den Tourismus haben könnte (Martin, 20I2).
} 


\section{Konstruktion und De-/Renationalisierung des neuseeländischen Kinos im Kontext der Globalisierung}

\section{I Einleitung}

Die Intensivierung der Globalisierungsprozesse in den letzten Jahrzehnten hat für die neuseeländische Filmindustrie weitreichende Folgen gehabt. Was aber hat sich genau in faktischer und subjektiver Hinsicht verändert und wie können diese Veränderungen erklärt bzw. interpretiert werden? Was für Auswirkungen haben diese Veränderungen auf das Konzept eines zeitgenössischen „nationalen neuseeländischen Kinos“? Der Vorgang der Globalisierung kann abwechselnd und oft sogar simultan zu De- sowie Renationalisierung führen, wobei Denationalisierung im Folgenden als ein nationale Konstrukte infrage stellender und schwächender Prozess, Renationalisierung hingegen als etwas, das nationale Konstrukte in ihrer Funktion bestärkt, verstanden wird. Globalisierung kann somit zum einen zu einer Destabilisierung des nationalen Gefüges führen, indem beispielsweise die Souveränität des Nationalstaates von transnational agierenden Unternehmen unterwandert wird. Andererseits kann diese Destabilisierung zu nationalen Maßnahmen führen, die darauf abzielen, eben diesen destabilisierenden Tendenzen entgegen zu wirken. Basierend auf dieser Prämisse ist die zentrale These dieses Kapitels, dass die genannten Prozesse unterschiedliche Auswirkungen auf den Komplex „nationales neuseeländisches Kino“ haben, je nachdem, welche Ebene betroffen ist und welcher Zeitraum betrachtet wird. Bevor im dritten Kapitel die ästhetische Rolle des Handlungsortes und der location auf der Produktions-, der para- und der metatextuellen Ebene ${ }^{\mathrm{II}}$ analysiert wird, soll in diesem Kapitel zuerst die grundlegende Bedeutung der Produktionsebene im Diskurs um ein nationales neuseeländisches Kino untersucht werden. Hierbei wird der Fokus auf die vier der Produktionsebene untergeordneten Ebenen bzw. Aspekte

\footnotetext{
${ }^{\text {II }}$ Genette definiert Paratext als Text, der den Haupttext (beispielsweise einen Roman) ergänzt und entweder vom Autor selbst oder von Dritten verfasst wurde, z. B. Vorwort, Nachwort oder Inhaltsverzeichnis. Das literaturwissenschaftliche Konzept des Paratextes lässt sich gut auf den Spielfilm übertragen; so können Interviews mit den Filmemachern und Making-Of's als eine Art „Vorwort“ bzw. „Nachwort“ eines Films aufgefasst werden. In Abgrenzung zu Metatexten, die sich auch negativ zum Haupttext verhalten können, beschreibt Genette Paratexte, die nicht vom Autor selbst verfasst wurden, als eine Art „Gefälligkeit“ dem Autor gegenüber (1989: 32I-323). Im Rahmen dieser Arbeit werden Texte Dritter dann als Paratexte definiert, wenn sie deutlich erkennbar als Bestandteil der Marketingstrategie erscheinen und demnach auch eine „Gefälligkeit“ darstellen. In der Regel ist dies bei MakingOf's und Interviews der Fall.

Auf der metatextuellen Ebene wird hingegen der Zuschauerdiskurs über die Filme erörtert, der im Gegensatz zu Paratexten keine „Gefälligkeit“ gegenüber den Filmemachern darstellen muss und daher auch sehr kritisch sein kann. Sowohl die para- als auch die metatextuelle Ebene bewegen sich außerhalb des Filmtextes selbst und können daher nicht unter die Filmtextebene subsumiert werden.
} 
„Produktionsfirma“, „Regisseur“, „Drehbuchautor“ und „location“ gelegt, da diese im genannten Diskurs die wichtigste Rolle spielen. ${ }^{\mathrm{I}}$

Der Prozess der Globalisierung wird mitunter als einseitiger Prozess der „Amerikanisierung“ der restlichen Welt, als eine Art US-„Kulturimperialismus“ aufgefasst, eine These, die im filmischen Bereich durch die Dominanz Hollywoods in vielen Ländern bestätigt zu werden scheint. Es gilt aber zu bedenken, dass es durchaus Widerstand gegen diese Art der Globalisierung gibt: So konnte Hollywood bis jetzt den von Bollywood dominierten indischen Filmmarkt kaum durchdringen, und in Ländern wie Frankreich oder Südkorea gibt es Filmquoten als „Anti-Hollywood“Maßnahmen. Auch wenn in Neuseeland keinerlei Filmquoten eingeführt wurden, weist das neuseeländische Kino durchaus Resistenzen gegen eine absolute Dominanz des Hollywoodkinos auf. Wie bei praktisch allen nationalen Konstrukten handelt es sich auch beim neuseeländischen Kino nicht von ein klar umrissenes, stabiles Konzept, sondern vielmehr etwas dynamisch Wandelbarem. Ich gehe von einer widersprüchlichen Entwicklung aus, bei der die genannten Unterebenen zum Teil in gegensätzliche Richtungen „auseinanderdriften“, aber auch zum Teil relativ stabil bleiben können, wenn sich die De- und Renationalisierungsprozesse, die auf eine Ebene einwirken, gleich stark sind und sich daher gegenseitig „relativieren“ bzw. aufheben. So sind meiner Ansicht nach die Erfolge transnationaler Produktionen wie The Piano, Lord of the Rings und Whale Rider nicht als Symptom eines einseitigen Prozesses der Denationalisierung auf allen Ebenen des neuseeländischen Kinos zu betrachten, da sich bei genauerer Betrachtung vielmehr graduelle Umformungen erkennen lassen, die in unterschiedliche Richtungen weisen und teilweise auch eindeutig renationalisierenden Charakters sind.

Durch meinen Fokus auf das neuseeländische Kino beziehe ich eine „nationale“ Perspektive, wobei die zunehmend transnationalere Ausrichtung vieler Bereiche des neuseeländischen Kinos berücksichtigt wird. Diese vorwiegend nationsfokussierte Perspektive wird mitunter kritisiert: So ist es nach Ansicht Durovicovas nicht ausreichend, sich auf nationale Kinos zu fokussieren, diese dann aber als „sich in Auflösung befindliche“ („under erasure“) Konstrukte darzustellen. Stattdessen wäre ihrer Ansicht nach ein Wechsel hin zu einer „zeitgemäßeren“ transnationalen Perspektive angebracht (2010: ix; sie spricht von einer „upgraded perspective“). Gegen diese Sichtweise ist aber

\footnotetext{
${ }^{12}$ Die ebenfalls interessante Kategorie der Schauspieler kann nicht berücksichtigt werden, da die notwendige Differenzierung zwischen „Hauptdarstellern“ und „Nebendarstellern“ sehr vage ist. Auch eine pauschale Feststellung der Nationalitäten sämtlicher Schauspieler, um dadurch das Abgrenzungsproblem Haupt-/Nebendarsteller zu umgehen, ist bei dem zu analysierenden Filmkorpus von rund 200 Filmen kaum zu bewältigen, zumal sich zu relativ unbekannten Schauspielern häufig keine detaillierteren Informationen zu ihrer Nationalität finden lassen.
} 
einzuwenden, dass der Fokus der Verfechter einer transnationalen Perspektive auf Regionen (z. B. Asien) oder Filmgenres keineswegs weniger problematisch als die Kategorie des „nationalen Kinos“ ist, da diese Kategorien ebenfalls Konstrukte sind, was aber häufig nicht weiter problematisiert wird. ${ }^{13}$ Die Idee eines transnationalen Kinos wird zwar nicht durch nationale Agenden forciert, ist aber letztendlich ebenso konstruiert wie die Idee eines nationalen Kinos, da weder auf der Produktionsebene noch auf der inhaltlichen Ebene eindeutig abgegrenzt werden kann, was noch national und was bereits transnational sein soll. Letztlich lassen deshalb auch Aufsatztitel wie „Brazilian Cinema“ (Vieira, 2010) oder „New Indian Cinema“(Desai, 2006) erkennen, dass die Kategorie des Nationalen auch in Arbeiten mit transnationalem Fokus noch eine Rolle spielt.

Deshalb sollten die Kategorien „national“ und „transnational“ auch nicht als gegensätzlich und einander ausschließend verstanden, sondern in einem dialogischen Verhältnis zueinander aufgefasst werden, wie Ezra argumentiert (2006: 4). Den genauen Bedeutungshorizont des Begriffs des „Transnationalen“ zu fassen, ist aufgrund seiner mittlerweile inflationären Verwendung schwierig. Im Rahmen dieser Arbeit wird das Transnationale als etwas begriffen, das das Nationale voraussetzt, zugleich aber auch transzendiert (Ezra 2006: 4) und einen Teilprozess der Globalisierung darstellt. Konsequent zu Ende gedacht würde eine vollständige Auflösung der Kategorie „national“ auch den Begriff „transnational“ überflüssig machen, da der Begriff bereits vom Wortsinn her immer in Bezug zum „Nationalen“ gesetzt wird.

Wenn ich mich auf das neuseeländische Kino konzentriere, nehme ich eine „nationale“, aber keineswegs eine „nationalistische“ Sichtweise ein. So soll in diesem Kapitel gerade nicht unterstellt werden, dass es einen generellen Konsens Neuseelands über „sein“ nationales Kino gäbe, was Higson als „the myth of consensus“ (1995: 273-274) bezeichnet. Vielmehr soll auch der Dissens über das, was nationales Kino ausmacht, untersucht werden. Higson scheint durch seinen Fokus auf den Pluralismus der Konstruktionen nationaler Kinos allerdings zu wenig herauszustreichen, dass es auf der anderen Seite durchaus auch einen Konsens über bestimmte Aspekte, was einen Film „national“ macht, geben kann. In der später dargestellten „Konsens-Dissens“-Analyse wird genauer auf diese Widersprüchlichkeiten eingegangen werden.

Denationalisierende und renationalisierende Prozesse werden im Rahmen dieser Arbeit als parallel ablaufend und sich zum Teil als gegenseitig verstärkend oder auch relativierend verstanden,

\footnotetext{
${ }^{\mathrm{I} 3}$ Für eine ausführliche Kritik des Genrebegriffs siehe Jason Mittell. „A Cultural Approach to Television Genre Theory“. Cinema Journal 40.3 (200I): 3-23. Print. In dem Artikel definiert Mittell Genres als „discoursive clusters“, die hohl und ohne intrinsischen Kern sind (II).
} 
was im Folgenden noch etwas eingehender erörtert werden soll: In der Forschung werden meist wirtschaftliche Prozesse als Initiator und Hauptmotor der Globalisierung betrachtet, die in der Folge auch Kultur und Politik beeinflussen. Globalisierung beschreibt letzten Endes die zunehmende und immer komplexer werdende weltweite Interdependenz von Ländern, Organisationen, usw., wobei die transnationalen Handlungen einer dieser Entitäten hierdurch weitreichende Folgen für viele andere Entitäten haben können. Transnational agierende Unternehmen bezeichnet Drechsel als global players (2000: 5). Zu den global players zählen viele, wenn nicht gar die meisten Filmproduzenten und auch zahlreiche Filmemacher und -mitarbeiter. Während heute größere Filmprojekte (so auch in Neuseeland) praktisch zwangsläufig auf transnationale Distribution angewiesen sind, da sie allein aus den durch das nationale Publikum generierten Umsätzen nicht einmal die Produktionskosten decken könnten, ist gerade die amerikanische Filmindustrie, die als eine der wenigen auch aus nationalen Einnahmen gewinnbringend produzieren könnte, am stärksten transnational ausgerichtet; dies gilt vor allem für das Hollywoodkino, das in den Filmwissenschaften oft als transnationales Kino per se angesehen wird (Ezra 2006: I).

Denationalisierenden Tendenzen stehen renationalisierende Tendenzen gegenüber bzw. werden dadurch erst erzeugt: Während viele Unternehmen zu global players geworden sind und die Idee des Nationalen für sie kaum mehr eine Rolle spielt, halten Politiker und kulturelle Institutionen in vielen Bereichen weiterhin oder gerade deswegen an der Idee des Nationalen fest (Drechsel 2000: 5). Die nationale Filmindustrie befindet sich quasi an der Schnittstelle von Kultur und Wirtschaft und wird oft für politische Zwecke eingebunden (beispielsweise durch staatliche Filmförderungsprogramme, aber auch Zensur), sodass sich hierdurch früher seltene Konstellationen ergeben, wie beispielsweise transnationale Filmproduktionen, die aber explizit nationale Themen behandeln. Dies lässt sich damit erklären, dass Nationen und deren Kulturen durch den verstärkten transnationalen Austausch in ein Konkurrenzverhältnis von nie da gewesener Intensität geraten, da durch den Wegfall protektionistischer Maßnahmen erst recht das eigene nationale Profil geschärft werden muss, um der mit der Globalisierung einhergehenden Tendenz der Nivellierung von Unterschieden entgegenzuwirken (Drechsel 2000: IO-2I). Gerade die Unterschiede zwischen nationalen Kulturen bilden heute ein wichtiges Kapital, das auch die nationalen Kinos und Hollywood regelmäßig in ihren Filmen nutzen und damit fortlaufend rekonstruieren. Diese filmische Thematisierung nationaler Eigenarten und Differenzen wird wiederum auch heute noch von Zuschauern in ihren Rezensionen aufgegriffen und diskutiert. Deshalb halte ich das Konzept des „nationalen 
Kinos“ für eine weiterhin sinnvolle, wenn auch nicht unproblematische Kategorie, die neben anderen durchaus berechtigten transnationalen Perspektiven auch heute noch eine wichtige Rolle in der filmwissenschaftlichen Forschung spielen sollte.

Wie kann nun nationales Kino definiert werden? Die Definition eines „nationalen Kinos“ setzt eine Definition von „Nation“ voraus, um nationales Kino dazu in Kontext setzen zu können. Die viel zitierte Theorie zur Nation von Benedict Anderson erweist sich auch im Rahmen dieser Arbeit als hilfreich. Anderson zufolge könnte man die Idee einer Nation als imagined community bezeichnen, einem aktiv konstruierten Gemeinschafts- und Zusammengehörigkeitsgefühl einer Gruppe von Menschen, die sich als relativ autonom und von anderen Nationen sowohl geografisch als auch kulturell (zumindest teilweise) abgegrenzt wahrnimmt (2006: 6). Die Frage, wie der Bevölkerung das Gefühl, zu jener Gemeinschaft (community) zu gehören, vermittelt wird, beantwortet Anderson mit dem Verweis auf die zentrale Rolle des print-capitalism für den Nation-BuildingProzess sowie die anschließende Aufrechterhaltung des Wir-Gefühls. Vor allem im Roman und dem Zeitungswesen erblickt er das Potenzial der massenmedialen Vermittlung einer nationalen Identität (2006: I8, 25). Der Spielfilm kann ebenfalls - und heute womöglich als noch bedeutsamerer - Vermittler dieser kollektiven Identität angesehen werden. Hierbei muss aber darauf hingewiesen werden, dass die Sicht der Filmemacher nicht unbedingt repräsentativ für die Gesamtbevölkerung sein muss, sondern vielmehr die Sicht der Filmemacher einen Teilausschnitt aus der komplexen und widersprüchlichen „Identität“ einer Nation darstellen, die nicht nur die nationalen Botschaften der Filme, sondern auch beispielsweise das im Rahmen eines nation brandings und in der Tourismuswerbung vermittelte Bild von Neuseeland teilweise inkorporiert hat.

Wenn im Rahmen dieser Arbeit von „Nation“ gesprochen wird, dann in erster Linie im Sinne einer kulturellen Rückbezüglichkeit auf den ursprünglichen Bezugsraum, in diesem Falle Neuseeland. Durch kulturelle Güter wie Filme, die als „national“ definiert werden, kann sich Neuseeland bezüglich seines Status als „Nation“ selbst vergewissern und dann wiederum „nationale“ Filme produzieren. Erst durch diese zirkuläre Wechselwirkung konstruiert Neuseeland sowohl sich als „Nation“ als auch seine „nationalen“ Filme. Der Konstruktionsakt des Nationalen manifestiert sich keineswegs nur in expliziten Akten, bei denen die Landesflagge an Nationalfeiertagen voller Pathos gehisst wird und Außenstehenden oft als sentimental oder gar radikal erscheint - ein Akt, den Michael Billig als hot nationalism bezeichnet (1995: 4). Viel bedeutsamer ist im Rahmen dieser Arbeit der alltägliche banal nationalism (Billig, 1995) oder „banale Nationalismus“, bei dem die na- 
tionalen Artefakte nur im Hintergrund erscheinen, dafür aber omnipräsent sind und von der eigenen Bevölkerung meist gar nicht als Zeichen des Nationalismus wahrgenommen werden, sondern als etwas Alltägliches, als etwas „Selbstverständliches“. Gerade diese „Natürlichkeit“ aber ist ein wichtiges Kennzeichen einer funktionierenden Ideologie. Diese unauffällige Form des Nationalismus ist prägend für die modernen neoliberalen Industriestaaten, so auch für Neuseeland, wobei sich insbesondere die Populärkultur - und damit auch der Spielfilm - sowohl inhaltlich als auch im Hinblick auf die Produktionshintergründe dieser Filme als gute Projektionsfläche für die Reproduktion nationaler Dogmen anbietet.

Bei der Abgrenzung eines Nationalstaats von anderen Nationalstaaten spielt die geografische Dimension eine bedeutende Rolle: Ein Staat legt sein Territorium in einem Rechtsakt genau fest, was sich wiederum darauf auswirkt, was die Bewohner dieses Nationalstaats als ihre „Heimat“ empfinden. In einem Spielfilm mit „nationalem Inhalt“ wird der Handlungsort filmgeografisch verortet, wobei die Filmlandschaft hierbei eine besondere Rolle spielt. Neben als „nationsspezifisch" erachteten Interaktionsweisen der Charaktere mit der Landschaft kann die Filmlandschaft auf ästhetischer Ebene mit als nationalspezifisch definierten Attributen wie indigener Flora und Fauna sowie besonderer kultureller Bauten versehen werden, um sie von der „typischen“ Landschaft anderer Länder abzugrenzen. Bei der Darstellung der neuseeländischen Landschaft sind deutliche Unterschiede zwischen neuseelandfokussierten Produktionen, d. h. Produktionen, die die neuseeländische Landschaft als neuseeländische Landschaft darstellen, und nicht-neuseelandfokussierten Produktionen, d.h. Produktionen, die mittels einer neuseeländischen location eine ausländische oder eine Fantasy-Landschaft simulieren, zu erkennen. Während in älteren nationalen Produktionen wie Vigil (1984) die nationalspezifischen Landschaftsattribute kaum hervorgehoben werden und die Landschaft daher fast „,anational“ erscheint, heben moderne, transnational produzierte Filme wie Whale Rider (2002) die nationalspezifischen Elemente expliziter hervor, um einem globalen Publikum den Reiz des Exotischen zu bieten und einen virtuellen Tourismus sowie die filmgeografische Erkundung fremder Länder zu ermöglichen (Zimmermann 2007: 58). Diese starke Betonung kann als symbolische Renationalisierung gedeutet werden, da gerade in Zeiten der tatsächlichen Auflösung nationaler Grenzen mehr auf die Besonderheiten von „Nationallandschaften“ (d. h. Landschaften, die als spezifisch für eine bestimmte Nation definiert werden) Wert gelegt wird als in früheren Filmen, als die Integrität der neuseeländischen Nation noch nicht oder nur wenig durch Nivellierungstendenzen infrage gestellt wurde. Zwar sind (Film-)Landschaften nicht 
per se national, sie werden aber durch Strategien der Filmemacher sowie der Rezeption der Zuschauer zu „nationalen“ Landschaften gemacht.

Die quasi seit ihren Anfängen stark transnational ausgerichtete Hollywoodindustrie verfolgt parallel dazu die Strategie einer bewussten „Anonymisierung“ bzw. „De-, und „Entlokalisierung“ der neuseeländischen Landschaft durch Ausblendung nationalspezifischer Aspekte und „Bereinigung“ von postkolonialen Problematiken, um sie dann als Projektionsfläche für nichtneuseeländische Handlungsorte wie beispielsweise „Mittelerde“ in Lord of the Rings zur Verfügung zu stellen und einem internationalen Massenpublikum zu präsentieren. Diese Verwendung der neuseeländischen Landschaft zur Simulation nicht-neuseeländischer (National-)Landschaften ist aber nicht zwangläufig ein einseitig denationalisierender Prozess, sondern kann durchaus renationalisierendes Potenzial haben, wobei hier von zentraler Bedeutung ist, ob der Zuschauer Kenntnis von der location hat und dadurch zwischen fiktionalem Handlungsort und realer location differenzieren kann. In letzterem Fall kann der Zuschauer die Filmlandschaft auch „gegen den Strich" lesen und als neuseeländische Landschaft rekonstruieren, was wiederum für den Filmtourismus relevant ist, worauf im dritten Kapitel intensiver eingegangen wird.

Während auf der filmtextuellen Ebene die Parameter für die Bestimmung der „Nationalspezifizität" eines Films immer auch subjektiv interpretiert werden müssen und eine empirische Analyse kaum zulassen, ist dies auf der hier zu analysierenden Produktionsebene durchaus möglich, da die Nationalitäten der Filmemacher und die Position der location grundsätzlich objektiv feststellbar sind. So kann ein Film wie Lord of the Rings auf der Produktionsebene als transnationale Koproduktion definiert werden, da die Produktionsfirmen zweier verschiedener Länder (New Line Cinema [USA] und Wingnut Films [Neuseeland]) den Film gemeinsam produziert haben. Allerdings stellt sich auch auf dieser Ebene die Frage, ab wann genau ein Film als transnational eingestuft werden kann und wann als nationale Produktion. Sollte beispielsweise ein Film, der fast vollständig in einem Land produziert wurde, an dem aber ein ausländischer Tontechniker mitgewirkt hat, bereits als „transnational“ eingestuft werden? Meiner Ansicht nach zeigt sich hieran, dass eine binäre Einteilung in „national“ oder „transnational“ zu rigide ist und stattdessen eine graduelle Einteilung wie beispielsweise „stark national“ und „schwach transnational ausgerichtet“ zu bevorzugen ist.

Zusammenfassend könnte eine „Idealdefinition“ des nationalen Kinos folgendermaßen formuliert werden: „Nationales Kino“ ist Kino, das national produziert wurde, mit einem filmi- 
schen Inhalt, der sich in kultureller, historischer und geografischer Hinsicht auf die eigene Nation bezieht. Sowohl durch die nationale Produktion als auch die nationalen Filminhalte wird das Gefühl einer imagined community erzeugt, und aufgrund dieser Eigenschaften wird dieses Kino von einem größtmöglichen Teil der Rezipienten als nationales Kino wahrgenommen. Ausgehend von diesem Idealtypus könnten graduelle Abweichungen dann als „Denationalisierung“ oder „Renationalisierung" interpretiert werden.

Wenn ich mich im Folgenden aus methodischen Gründen auf die Analyse die Produktionsebene beschränke, bedeutet dies keineswegs, dass der Inhalt bei der Beurteilung der „Nationalität" eines Films keine Rolle spielen würde. Der Filminhalt ist zweifellos wichtig bei der Beurteilung eines Films als „national“/“nicht-national“. Letztendlich kann weder durch die isolierte Analyse der Filminhalte, noch durch eine isolierte Analyse der Produktionsebene ein umfassender Gesamtblick auf das neuseeländische Kino geliefert werden. Der somit durch den Fokus auf die Produktionsebene notwendigerweise eingeschränkte Blick auf den Komplex „neuseeländisches Kino“ soll durch einige im Anschluss an die Analyse im Punkt 2.4 folgende Überlegungen zur De-/Renationalisierung von Filminhalten erweitert werden, auch wenn diese keine tiefer gehenden Analysen ersetzen können. In dieser Hinsicht haben unter anderem Conrich (2008) und Leotta (20II) tiefer gehende, selektive Analysen filmischer Inhalte neuseeländischer Filme geleistet, eine übergreifende Analyse der Inhalte sämtlicher neuseeländischer Filme hinsichtlich nationaler Fragestellungen ist bisher aber noch nicht geleistet worden. Hier bestünde durchaus Potenzial für zukünftige Forschungsarbeiten. Das vorliegende Kapitel versteht sich umgekehrt als möglichst umfassende Beleuchtung des bislang zugunsten inhaltlicher Analysen eher vernachlässigten Bereichs der Produktionsebene neuseeländischer Filme.

\subsection{Methodik}

Im Folgenden wird nun ein auf den vorangegangenen Überlegungen basierendes FünfEbenen-Modell erläutert, das folgende Voraussetzungen erfüllen soll:

I. Die Unterebenen sollen möglichst klar voneinander abgrenzbar sein

2. Die einzelnen Parameter sollen quantitativ und wo möglich auch qualitativ bewertet werden 3. Es soll eine möglichst genaue Beurteilung der Veränderungen der Ausrichtung des neuseeländischen Kinos (von „stark national" bis „stark transnational”) im Zeitraum 1977-2008 gewährleistet werden 


\begin{tabular}{|l|}
\hline Produktions e be ne \\
\hline 1. Nationalität der Produktionsfirmen \\
\hline 2. Nationalität des Regisseurs \\
\hline 3. Nationalität des Drehbuchautors \\
\hline 4. Neuseeländische / ausländische location \\
\hline 5. Verhältnis neuseeländische / nicht-neuseeländische \\
Filmproduktionen in neuseeländischen Kinos \\
(100 erfolgreichsten Filme im Jahr)
\end{tabular}

Tabelle 2.I: Fünf-Ebenen-Modell

Für das Modell ist jede Unterebene möglichst eindeutig gewählt worden. Jede Unterebene wird zuerst getrennt untersucht und anschließend den anderen Unterebenen vergleichend gegenübergestellt. Ziel der Analyse ist die Beantwortung folgender Fragestellungen:

I. Bis zu welchem Zeitpunkt kann das Aufkommen transnationaler Produktionen in der neuseeländischen Filmindustrie zurückverfolgt werden? Gab es sie möglicherweise schon immer?

2. Welche Unterebenen haben sich deutlich verändert und welche sind weitgehend konstant geblieben? Welche Trends sind erkennbar?

3. Sind bestimmte Kräfte bzw. Auslöser für eine verstärkte transnationale Ausrichtung oder umgekehrt die Rückbesinnung auf die nationale Sphäre erkennbar?

4. Wie groß ist die Bedeutung der Produktionsebene bei der Konzeption des neuseeländischen Kinos?

5. Haben alle Unterebenen das gleiche Gewicht bei der Zuordnung eines Films zum nationalen neuseeländischen Kino oder sind bestimmte Unterebenen hierbei wichtiger als andere?

Um diese Fragestellungen zu beantworten, werden die nachfolgend erläuterten Unterebenen quantitativ analysiert: Hinsichtlich der Nationalitäten der Filmemacher beschränke ich mich auf diejenige der Produktionsfirmen, des Regisseurs und des Drehbuchautors, da diese in der industriellen Praxis als wichtigste Mitarbeiter bei der Filmproduktion beurteilt werden und den Filminhalt in aller Regel am stärksten beeinflussen. An dieser Stelle sei bemerkt, dass Nationalität und nationale Identität einer Person nicht immer „kongruent" zueinander sein müssen. So unterscheidet sich beispielsweise die subjektive Vorstellung, was es heißt, „ein Neuseeländer" zu sein, deutlich, je nachdem, ob es sich um ein Individuum, das sich der kulturellen Gruppe der Māori zuordnet, um ein seit sechs Generationen in Neuseeland lebendes britischstämmiges Individuum 
oder um einen seit lediglich einem halben Jahr in Neuseeland eingebürgerten Deutschen handelt. Je nach nationalem Hintergrund importiert ein Immigrant mehr oder weniger großen kulturellen „Ballast“ in das Immigrationsland, wobei die Art der Rekonfiguration der eigenen Identität im Rahmen der Austarierung zwischen eigener und fremder Kultur stark vom persönlichen Integrations- bzw. Assimilationswillen abhängt. Hinsichtlich der Filmproduktion bedeutet dies beispielsweise, dass ein neuseeländischer Regisseur nicht zwangsläufig eine nationale Agenda verfolgen muss, sondern eine weniger „idealistische", auf maximalen finanziellen Ertrag ausgerichtete Produktionsweise bevorzugen kann, die meist stärker auf ein transnationales Publikum gerichtet ist.

Des Weiteren sollen die Unterebenen auch hinsichtlich des Anteils an Filmmitarbeitern analysiert werden, die sich kulturellen Minderheiten zuordnen, um beurteilen zu können, inwiefern der Markt von Pākeh $\bar{a}^{-I 4}$ dominiert wird und damit unter Umständen als eine Form kulturellindustrieller Hegemonie gedeutet werden könnte. Damit lässt sich in einer Gesamtschau die ethnische Zusammensetzung der neuseeländischen Filmindustrie und deren Veränderungen im Laufe der Zeit beurteilen.

Die Unterebene „location" ist nicht mit dem Handlungsort gleichzusetzen, da insbesondere im Falle der Simulation eines ausländischen Handlungsortes durch eine neuseeländische location eine filmgeografische Inkongruenz vorliegt. Mit der Bezeichnung „Handlungsort“ ist im Gegensatz zur location, wo der Film gedreht wurde, der Ort bzw. die Welt gemeint, wo sich das narrativische Geschehen des Films ereignet. Handlungsorte können realitätsbasiert, ${ }^{15}$ zeitgenössisch, historisch, futuristisch oder rein fiktiv sein. Die „Nationalität“ des Handlungsortes wird auch deshalb nicht im Rahmen des ersten Kapitels analysiert werden, weil das filmische Geschehen oftmals gar nicht genau verortet ist und das Geschehen damit fast „überall“ sein könnte, wohingegen die locations immer eindeutig bestimmbar und damit quantitativ erfassbar sind. ${ }^{16}$ Vergleichend wird schließlich das Verhältnis zwischen nicht- neuseeländischen und neuseeländischen Filmen am Kinomarkt in Neuseeland betrachtet, wobei der Fokus umsatzorientiert ist und nicht die absolute Filmanzahl verglichen wird.

Generell wird bei der Analyse der Unterebenen der Zeitraum von 1977-2008 betrachtet werden, sofern vollständige Daten dazu vorliegen. Leider ist dies nicht immer möglich, da einige

\footnotetext{
${ }^{14}, P \bar{a} k e h \bar{a}$ “ ist in der Maorisprache die Bezeichnung für Nicht-Maori und meint in erster Linie europäischstämmige Neuseeländer. ${ }^{15}$ Mit „realitätsbasiert“ ist der Bezug zu einem real existierenden Ort oder einem Ort, der früher einmal existiert hat, gemeint. „Realitätsbasiert“ ist somit das Gegenteil von „fiktiv“. Da filmische Handlungsorte immer virtuell sind und sich zwar auf einen realen Ort beziehen, nicht aber dieser Ort selbst sein können, wäre die verkürzte Bezeichnung „realer“ Handlungsorte unpräzise. ${ }^{16}$ Das Spannungsverhältnis zwischen der Geografie des Handlungsorts und der location wird im dritten Kapitel erörtert werden.
} 
Statistiken nicht den gesamten Zeitraum abdecken. Als primäre Quellen und Referenzen werden die in den USA betriebene Filmdatenbank IMDb (imdb.com) und Box Office Mojo (boxofficemojo.com) herangezogen.

Bevor mit der Analyse der Produktionsebene begonnen werden kann, stellt sich eine zentrale methodische Frage: Wie kann aus der oben genannten idealtheoretischen Definition des nationalen Kinos ein konkretes Filmoeuvre als Analysegrundlage für die Produktionsebene gebildet werden, das der Praxisrealität gerecht wird und wirklich aussagekräftig ist, d. h. nicht aufgrund normativer Überlegungen „aus dem theoretischen Vakuum“ heraus gebildet wurde?

Die Idealdefinition wirft in Bezug auf die Analyse der Produktionsebene zwei Problematiken auf: Zum einen lassen sich aus ihr nicht die konkreten Kriterien bzw. Prinzipien ableiten, die in der Praxis angewendet werden, um zu entscheiden, ob ein bestimmter Film, der einen „Grenzfall“ darstellt, noch in das Filmoeuvre neuseeländischer Filme aufgenommen oder als vorwiegend ausländische Produktion aus dem Oeuvre ausgeschlossen werden soll. Die Ermittlung konkreter, graduell ausgerichteter Prinzipien ist aber insofern notwendig, als dass es letztendlich keinen Film gibt, der der Idealdefinition vollständig entspricht. Die strikte Ausfilterung nicht „idealtypischer“ Filme würde die Bildung eines Filmoeuvres daher gar nicht erst ermöglichen.

Zum anderen würde aber auch die normative Festlegung eigener Ein- und Ausschlusskriterien gewissermaßen zu einem „Zirkelbezug“ führen und keine wirkliche Aussagekraft besitzen: Bei einer Festlegung auf relativ strenge Kriterien würden nur Filme aufgenommen, die zu einem großen Teil in und von Neuseeland produziert wurden. Umgekehrt würde eine weite Auslegung von Kriterien zu einem deutlich größeren Filmkorpus führen, da auch Filme aufgelisztet würden, die signifikante ausländische Anteile aufweisen (beispielsweise ein Film, in dem nur der Drehbuchautor neuseeländisch ist, Produktionsfirma, Regisseur und location hingegen ausländisch). Durch eine solche Vorgehensweise würde aber von vornherein feststehen, in welchem Ausmaß das neuseeländische Kino „denationalisiert“, „stabil geblieben“ oder „renationalisiert“ ist: Bei entsprechend strengen Kriterien würde das neuseeländische Kino entsprechend „stabil“ oder auch „renationalisiert“ erscheinen, wohingegen eine weite Auslegung zu dem Ergebnis einer „signifikanten Denationalisierung " führen würde. Somit würde eine normative Festlegung der Kriterien lediglich eine weitere Variante der Konstruktion des nationalen neuseeländischen Kinos hervorbringen und damit selbst Teil des nationalen Konstruktionsprozesses werden. 
Ziel dieser Arbeit ist es aber, sich von dem Konstruktionsprozess selbst zu distanzieren und diesen aus einer möglichst „neutralen“ Perspektive zu analysieren. Wie kann dies bewerkstelligt werden? Eine Möglichkeit wäre, einen bereits bestehenden, konkreten und vor allem „autoritativen“ Kriterienkatalog, der von einer anerkannten „nationalen Autorität“ erstellt worden ist, als Ausgangspunkt zu nehmen. Hierfür böte sich der im New Zealand Film Commission Act 1978 aufgeführte Kriterienkatalog an, wonach die Kriterien für die Auswahl von Filmen gemäß \$18.2 von dem Filminhalt, der location, den Nationalitäten der Filmemacher und -mitarbeiter und der Herkunft finanzieller Mittel abhängen sollen („New Zealand Film Commission Act 1978“: 8-9). Es wird aber nicht präzisiert, wie die vorgenannten Kriterien zu gewichten sind und welche konkreten Konstellationen einen „significant New Zealand content“ (8) darstellen und welche nicht. Letztendlich solle in einer Gesamtbetrachtung ermittelt werden, ob dieser „significant New Zealand content" vorliege, was aber im Ermessen der New Zealand Film Commission selbst liegt und daher eine unabhängige Anwendung der Kriterien unmöglich macht, sofern man nicht wieder auf die eigene Subjektivität zurückfallen möchte.

Da es demnach keine expliziten, konkret anwendbaren Richtlinien gibt, und es weder in der Theorie noch in der Praxis einen Konsens darüber gibt, was „nationales Kino“ genau ist und was nicht, soll hier ein anderer Weg beschritten werden, nämlich eine empirische Analyse aller verfügbaren „Filmkataloge“ ${ }^{17}$ um daraus mittels einer „Konsens-Dissens-Analyse“ diejenigen Filme zu ermitteln, die in mindestens einem Katalog als neuseeländischer Film eingestuft werden. Hierbei stellen die Filmkataloge verschiedene Konstruktionsvarianten des nationalen neuseeländischen Kinos dar, die durch eine Auflistung der betreffenden Filme konkretisiert worden sind, d. h. nicht nur abstrakte Konstruktionen darstellen. Diese Pluralität bestätigt, dass es letztlich nicht das nationale Kino, sondern verschiedene nationale Kinos innerhalb einer Nation gibt (Hayward 1993: I4).

Während es diverse Literatur über einzelne als für das neuseeländische Kino „exemplarisch“ erachtete Filme oder Filmgruppen gibt (d.h. im Endeffekt meist nur die erfolgreichsten und presti-

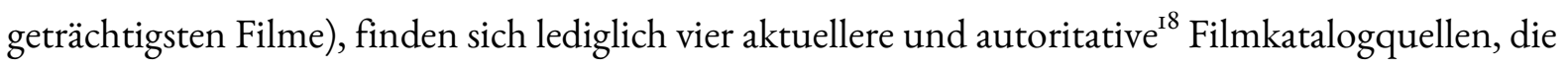

\footnotetext{
${ }^{17}$ Mit „Filmkatalog“ ist hier eine Filmliste sämtlicher als neuseeländisch erachteter oder mit Neuseeland assoziierter Filme gemeint, die sich beispielsweise in einem Buch als „Anhang“ oder auch als Online-Quelle findet.

${ }^{18}$ Auch Wikipedia bietet einen Filmkatalog über neuseeländische Filme an („List of New Zealand films“ unter http://en.wikipedia.org/wiki/List_of_New_Zealand_films). Da Wikipedia-Artikel von jedermann jederzeit revidiert werden können, gelten diese Artikel im wissenschaftlichen Diskurs als nicht zitierfähig. Deshalb wird auch im Rahmen dieser Arbeit der Wikipedia-Filmkatalog aufgrund seiner mangelnden „Autorität“ nicht berücksichtigt werden. Dennoch bietet auch dieser Katalog einen interessanten Überblick über neuseeländische Filme, wobei sich auf der Wikipedia-Diskussionseite („Talk“) zum Artikel unter dem Abschnitt „To Qualify as a New Zealand film“ ein reger Diskurs gebildet hat, welche Filme als neuseeländische Filme in die Liste aufgenommen werden und welche Kriterien hierbei gelten sollen (http://en.wikipedia.org/wiki/Talk:List_of_New_Zealand_films, Zugriff 5.4.20I5).
} 
einen Anspruch auf Vollständigkeit erheben und damit den Versuch, jeden als neuseeländisch erachteten Film sowie auch lediglich mit Neuseeland in Verbindung gebrachte ausländische Filme aufzulisten und damit ein Gesamtbild des neuseeländischen Kinos und seines „Umfeldes“ zu erfassen:

I. Internet Movie Database (imdb.com)

2. „Filmography: New Zealand Feature and Television Films (including related productions)“ in Contemporary New Zealand Cinema (Conrich/Murray 2008: 239-253, Anhang) 3. „Feature Films Made In New Zealand Since 1939“ (New Zealand Film Commission, 2012)

4. New Zealand Film: 1912-1996(Martin/Edwards, 1997; zusätzlich ausführliche Besprechung der einzelnen Filme)

Die zu analysierenden Filmkataloge illustrieren die große Bandbreite an Konstruktionsmöglichkeiten eines nationalen neuseeländischen Kinos: Während die Imdb eine große Filmdatenbank ist, die Filme aus aller Welt relativ „neutral“ nach ihrem „country of origin“ kategorisiert und dabei zumindest vorgibt, allein auf die Nationalität der Produktionsfirmen abzustellen, ${ }^{19}$ vertritt die New Zealand Film Commission (NZFC) einen ausgesprochen „nationalistischen“ Ansatz, wodurch die Filmauswahl interessanterweise deutlich arbiträrer als bei den anderen Filmkatalogen erscheint, da mitunter Filme mit praktisch identischem Produktionshintergrund einmal als „neuseeländisch“, ein anderes Mal als „ausländisch“ eingestuft werden ${ }^{20}$ - und dies, obwohl die NZFCListe durch ihren Verweis auf §I8.2 des New Zealand Film Commission Act (s. o.) eine scheinbar besonders große Eindeutigkeit und Klarheit der Zuordnungskriterien suggeriert. Demgegenüber zeigen sowohl Conrich als auch Martin die größte Selbstreflexivität bezüglich der Schwierigkeit, einzelne Filme als konkret neuseeländisch/nicht-neuseeländisch zu klassifizieren, was bei der NZFC fehlt.

Die Quelle New Zealand Film: 1912-1996 von Martin/Edwards deckt nur den hier relevanten Zeitraum von 1977-1996 ab, soll aber dennoch - aufgrund ihrer Bedeutung - bis zum Jahr 1996 mit berücksichtigt werden. Bevor nun die Konsens-Dissens-Analyse und die Ergebnisse näher erörtert werden, soll noch einmal der Bezug zur eigenen „Idealdefinition“ rekapituliert werden: Wäh-

\footnotetext{
${ }^{19}$ Neben dem Herkunftsland werden die Filme auch nach anderen Kriterien sortiert, wie beispielsweise Genre, Umsatz, etc. ${ }^{2 \circ}$ So wird The Piano von der NZFC als neuseeländischer Film eingestuft, während bei Mesmerized durch den Zusatz „locations“ suggeriert wird, dass der Film lediglich in Neuseeland gedreht worden, aber an sich eine ausländische Produktion sei und folglich nicht als neuseeländischer Film eingestuft werden könne.
} 
rend die Idealdefinition sämtliche Aspekte des Konstrukts des „nationalen neuseeländischen Kinos" aufgreift, soll in diesem Kapitel nur der Teilaspekt der Produktionsebene betrachtet werden, da die in der Definition zusätzlich angeführten inhaltlichen Aspekte wie die Vermittlung kultureller, historischer und geografischer Aspekte ${ }^{21}$ kaum quantifiziert werden können. Insofern soll hier nicht die Re- oder Denationalisierung der filmischen Inhalte analysiert werden, sondern sich auf die Produktionsebene konzentriert werden. Wie erwähnt werden aber im Anschluss an die Produktionsebenenanalyse einige Überlegungen zur De-/Renationalisierung filmischer Inhalte angestellt werden.

Im Folgenden ist die Konsens-Dissens-Analyse tabellarisch dargestellt:

${ }^{21}$ D. h. Handlungsort, aber nicht location. 


\begin{tabular}{|c|c|c|c|}
\hline \multicolumn{4}{|c|}{ Tabelle 2.2: Konsens-Dissens-Analyse } \\
\hline Film & Jahr & 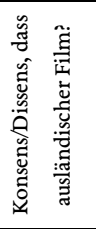 & 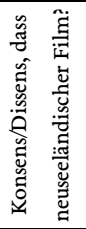 \\
\hline$B \in$ & 2008 & Konsens & \\
\hline $\begin{array}{l}\text { THE GHRONICLES OF NARNIA- } \\
\text { PRINCE CASPIAN }\end{array}$ & 2008 & Konsens & \\
\hline BRIDE FLIGHT & 2008 & Konsens & \\
\hline A SONG OF GOOD & 2008 & & Konsens \\
\hline APRON STRINGS & 2008 & & Konsens \\
\hline DEAN SPANLEY & 2008 & & Konsens \\
\hline JINX SISTER & 2008 & & Konsens \\
\hline LAST OF THE LIVING & 2008 & & Konsens \\
\hline LOVE HAS NO LANGUAGE & 2008 & & Konsens \\
\hline $\begin{array}{l}\text { SECOND-HAND WEDDING / GARAGE } \\
\text { SALE }\end{array}$ & 2008 & & Konsens \\
\hline SHOW OF HANDS & 2008 & & Konsens \\
\hline THE MAP READER & 2008 & & Konsens \\
\hline BRIDGE TOTERABITHIA & 2007 & Konsens & \\
\hline 30 DAYS OF NIGHT & 2007 & Dissens & Dissens \\
\hline $\begin{array}{l}\text { THE WATERHORSE: LEGEND OF THE } \\
\text { DEEP }\end{array}$ & 2007 & Dissens & Dissens \\
\hline DOWN BY THE RIVERSIDE & 2007 & & Konsens \\
\hline EAGLE VS. SHARK & 2007 & & Konsens \\
\hline KISSY KISSY & 2007 & & Konsens \\
\hline MEN SHOULD'NT SING & 2007 & & Konsens \\
\hline THE DEVIL DARED ME TO & 2007 & & Konsens \\
\hline THE FERYMAN & 2007 & & Konsens \\
\hline THE LAST GREAT SNAIL CHASE & 2007 & & Konsens \\
\hline THE LAST MAGIC SHOW & 2007 & & Konsens \\
\hline THE TATTOOIST & 2007 & & Konsens \\
\hline $\begin{array}{l}\text { WE'RE HERE TO HELP / BE VERY } \\
\text { AFRAID }\end{array}$ & 2007 & & Konsens \\
\hline WHEN NIGHT FALLS & 2007 & & Konsens \\
\hline BLACK SHEEP & 2006 & & Konsens \\
\hline EVENT I6 & 2006 & & Konsens \\
\hline NO. 2 & 2006 & & Konsens \\
\hline OUT OF THE BLUE & 2006 & & Konsens \\
\hline PERFECT CREATURE & 2006 & & Konsens \\
\hline SIONE'S WEDDING & 2006 & & Konsens \\
\hline THE WAIMATE CONSPIRACY & 2006 & & Konsens \\
\hline WAIT UP HARRIET & 2006 & & Konsens \\
\hline
\end{tabular}

\begin{tabular}{|c|c|c|c|}
\hline ANTARETHGJOURAAL (NAMGEUKH) & 2005 & Konsens & \\
\hline THE WATER GIANT (MEESHEE) & 2005 & Konsens & \\
\hline WITHOUT A PADDLE & 2005 & Dissens & Dissens \\
\hline $\begin{array}{l}\text { THE CHRONICLES OF NARNIA: THE } \\
\text { LION, THE WITCH \& THE } \\
\text { WARDROBE }\end{array}$ & 2005 & Dissens & Dissens \\
\hline 5O WAYS OF SAYING FABULOUS & 2005 & & Konsens \\
\hline BOOGEYMAN & 2005 & Dissens & Dissens \\
\hline HIDDEN & 2005 & & Konsens \\
\hline KING KONG & 2005 & & Konsens \\
\hline LUELLA MILLER & 2005 & & Konsens \\
\hline MEET ME IN MIAMI & 2005 & Dissens & Dissens \\
\hline MEMORIES OF TOMORROW & 2005 & & Konsens \\
\hline QUIET NIGHT IN & 2005 & & Konsens \\
\hline RIVER QUEEN & 2005 & & Konsens \\
\hline THE WORLD'S FASTEST INDIAN & 2005 & & Konsens \\
\hline TYRANNICAL LOVE & 2005 & & Konsens \\
\hline $\begin{array}{l}\text { THE LAND HAS EYES (PEAR TA MA } \\
\text { 'ON MAF) }\end{array}$ & 2004 & Konsens & \\
\hline INITE & 2004 & & Konsens \\
\hline FRACTURE / CRIME STORY & 2004 & & Konsens \\
\hline IN MY FATHER'S DEN & 2004 & & Konsens \\
\hline MURMURS & 2004 & & Konsens \\
\hline OFFENSIVE BEHAVIOUR & 2004 & & Konsens \\
\hline SPOOKED & 2004 & & Konsens \\
\hline THE LAST SAMURAI & 2003 & Dissens & Dissens \\
\hline CHRISTMAS & 2003 & & Konsens \\
\hline FOR GOOD & 2003 & & Konsens \\
\hline GUPTA VERSUS GORDON & 2003 & & Konsens \\
\hline I THINK I'M GOING & 2003 & & Konsens \\
\hline KOMBI NATION & 2003 & & Konsens \\
\hline $\begin{array}{l}\text { LORD OF THE RINGS: THE RETURN } \\
\text { OF THE KING }\end{array}$ & 2003 & & Konsens \\
\hline $\begin{array}{l}\text { NEMESIS GAME / PAPER, SCISSORS, } \\
\text { STONE }\end{array}$ & 2003 & & Konsens \\
\hline ORPHANS AND ANGELS & 2003 & & Konsens \\
\hline PERFECT STRANGERS & 2003 & & Konsens \\
\hline THE LOCALS & 2003 & & Konsens \\
\hline $\begin{array}{l}\text { WHY I CAN'T STOP THIS } \\
\text { UNCONTROLLABLE DANCING }\end{array}$ & 2003 & & Konsens \\
\hline WOODENHEAD & 2003 & & Konsens \\
\hline BLESSED & 2002 & & Konsens \\
\hline CUPID'S PREY & 2002 & & Konsens \\
\hline
\end{tabular}




\begin{tabular}{|c|c|c|c|}
\hline $\begin{array}{l}\text { LORD OF THE RINGS: THE TWO } \\
\text { TOWERS }\end{array}$ & 2002 & & Konsens \\
\hline OFF. & 2002 & & Konsens \\
\hline THE MĀORI MERCHANT OF VENICE & 2002 & & Konsens \\
\hline THIS IS NOT A LOVE STORY & 2002 & & Konsens \\
\hline TONGAN NINJA & 2002 & & Konsens \\
\hline TOY LOVE & 2002 & & Konsens \\
\hline WHALE RIDER & 2002 & & Konsens \\
\hline CROOKED EARTH & 2001 & & Konsens \\
\hline HER MAJESTY & 2001 & Dissens & Dissens \\
\hline KIDS WORLD & 2001 & & Konsens \\
\hline $\begin{array}{l}\text { LORD OF THE RINGS: FELLOWSHIP } \\
\text { OF THE RING }\end{array}$ & 2001 & & Konsens \\
\hline RAIN & 2001 & & Konsens \\
\hline SNAKESKIN & 2001 & & Konsens \\
\hline STICKMEN & 2001 & & Konsens \\
\hline THE WAITING PLACE & 2001 & & Konsens \\
\hline $\begin{array}{l}\text { WHEN STRANGERS APPEAR / SHEA- } \\
\text { RER'S BREAKFAST }\end{array}$ & 2001 & & Konsens \\
\hline ШERTHGAL LIMIT & 2000 & Konsens & \\
\hline HOPELESS & 2000 & & Konsens \\
\hline JUBILEE & 2000 & & Konsens \\
\hline SAVAGE HONEYMOON & 2000 & & Konsens \\
\hline SHIFTER & 2000 & & Konsens \\
\hline THE FEATHERS OF PEACE & 2000 & & Konsens \\
\hline $\begin{array}{l}\text { THE IRREFUTABLE TRUTH ABOUT } \\
\text { DEMONS }\end{array}$ & 2000 & & Konsens \\
\hline THE PRICE OF MILK & 2000 & & Konsens \\
\hline WILD BLUE & 2000 & & Konsens \\
\hline CHANNELLING BABY & 1999 & & Konsens \\
\hline I'LL MAKE YOU HAPPY & 1999 & & Konsens \\
\hline MAGIK AND ROSE & 1999 & & Konsens \\
\hline SCARFIES / CRIME IOI & 1999 & & Konsens \\
\hline THE LUNATIC'S BALL & 1999 & & Konsens \\
\hline UNCOMFORTABLE COMFORTABLE & 1999 & & Konsens \\
\hline $\begin{array}{l}\text { WHAT BECOMES OF THE BROKEN } \\
\text { HEARTED? }\end{array}$ & 1999 & & Konsens \\
\hline MEMORY AND DESIRE & 1998 & & Konsens \\
\hline THE CLIMB & 1998 & Dissens & Dissens \\
\hline VIA SATELLITE & 1998 & & Konsens \\
\hline WHEN LOVE COMES & 1998 & & Konsens \\
\hline ABERRATION & 1997 & & Konsens \\
\hline SAVING GRACE & 1997 & & Konsens \\
\hline
\end{tabular}

\begin{tabular}{|c|c|c|c|}
\hline THE UGLY & 1997 & & Konsens \\
\hline THE WHOLE OF THE MOON & 1997 & & Konsens \\
\hline $\begin{array}{l}\text { TOPLESS WOMEN TALK ABOUT THEIR } \\
\text { LIVES }\end{array}$ & 1997 & & Konsens \\
\hline BROKEN ENGLISH & 1996 & & Konsens \\
\hline CHICKEN & 1996 & & Konsens \\
\hline THE FRIGHTENERS & 1996 & & Konsens \\
\hline BONJOUR TIMOTHY & 1995 & & Konsens \\
\hline FLIGHT OF THE ALBATROSS & I995 & Dissens & Dissens \\
\hline $\begin{array}{l}\text { THE OFFERING / SHADOW OF THE } \\
\text { PEPPER TREE / ECLIPSE }\end{array}$ & 1995 & & Konsens \\
\hline HEAVENLY CREATURES & 1994 & & Konsens \\
\hline JACK BROWN GENIUS & 1994 & & Konsens \\
\hline LOADED & 1994 & & Konsens \\
\hline ONCE WERE WARRIORS & 1994 & & Konsens \\
\hline THE LAST TATTOO & 1994 & & Konsens \\
\hline DESPERATE REMEDIES & 1993 & & Konsens \\
\hline JACK BE NIMBLE & 1993 & & Konsens \\
\hline THE PIANO & 1993 & & Konsens \\
\hline TRAFFIC ISLAND & 1993 & & Konsens \\
\hline ABSENT WITHOUT LEAVE & 1992 & & Konsens \\
\hline ALEX & 1992 & & Konsens \\
\hline BRAINDEAD & 1992 & & Konsens \\
\hline CHUNUK BAIR & 1992 & & Konsens \\
\hline CRUSH & 1992 & & Konsens \\
\hline $\begin{array}{l}\text { GRAMPIRE / MOONRISE / MY } \\
\text { GRANDPA IS A VAMPIRE }\end{array}$ & 1992 & & Konsens \\
\hline SECRETS / ONE CRAZY NIGHT & 1992 & & Konsens \\
\hline THE FOOTSTEP MAN & 1992 & & Konsens \\
\hline OLD SCORES & 1991 & & Konsens \\
\hline TE RUA / THE STONE HOUSE / THE PIT & I99I & & Konsens \\
\hline THE END OF THE GOLDEN WEATHER & I991 & & Konsens \\
\hline AN ANGEL AT MY TABLE & 1990 & & Konsens \\
\hline MEET THE FEEBLES & 1990 & & Konsens \\
\hline RUBY AND RATA & 1990 & & Konsens \\
\hline THE RETURNING & 1990 & & Konsens \\
\hline USER FRIENDLY & 1990 & & Konsens \\
\hline CHILL FACTOR & 1989 & & Konsens \\
\hline FLYING FOX IN A FREEDOM TREE & 1989 & & Konsens \\
\hline ZILCH! & 1989 & & Konsens \\
\hline $\begin{array}{l}\text { THE RESCUE /SEAL KIDS/OPERATH- } \\
\text { ON PHOENIX }\end{array}$ & 1988 & Konsens & \\
\hline WILLOW & 1988 & Dissens & Dissens \\
\hline
\end{tabular}




\begin{tabular}{|c|c|c|c|}
\hline NEVER SAY DIE & 1988 & & Konsens \\
\hline A SOLDIERS TALE & 1988 & & Konsens \\
\hline MAURI & I988 & & Konsens \\
\hline SEND A GORILLA & 1988 & & Konsens \\
\hline THE NAVIGATOR & 1988 & & Konsens \\
\hline BAD TASTE & 1987 & & Konsens \\
\hline FOOTROT FLATS & 1987 & & Konsens \\
\hline ILLUSTRIOUS ENERGY & 1987 & & Konsens \\
\hline NGATI & 1987 & & Konsens \\
\hline STARLIGHT HOTEL & 1987 & & Konsens \\
\hline $\begin{array}{l}\text { MAD MISSION 4: YOUNEVER DIE } \\
\text { (ZUUE /AGESGOPLAGES/PANDA } \\
\text { (ZUAIAIDANG ZHI QIANLIJIU } \\
\text { GHAIPO) }\end{array}$ & 1986 & Konsens & \\
\hline ARRIVING TUESDAY & 1986 & & Konsens \\
\hline BRIDGE TO NOWHERE & 1986 & & Konsens \\
\hline MESMERIZED / SHOCKED & 1986 & Dissens & Dissens \\
\hline QUEEN CITY ROCKER & 1986 & & Konsens \\
\hline CAME A HOT FRIDAY & 1985 & & Konsens \\
\hline DANGEROUS ORPHANS & 1985 & & Konsens \\
\hline KINGPIN & 1985 & & Konsens \\
\hline LEAVE ALL FAIR & 1985 & & Konsens \\
\hline MR WRONG / DARK OF THE NIGHT & 1985 & & Konsens \\
\hline SHAKER RUN & 1985 & & Konsens \\
\hline SHOULD I BE GOOD? & 1985 & & Konsens \\
\hline SYLVIA & 1985 & & Konsens \\
\hline THE QUIET EARTH & 1985 & & Konsens \\
\hline THE BOUNTY & 1984 & Dissens & Dissens \\
\hline CONSTANCE & 1984 & & Konsens \\
\hline DEATH WARMED UP & 1984 & & Konsens \\
\hline HEART OF THE STAG & 1984 & & Konsens \\
\hline OTHER HALVES & 1984 & & Konsens \\
\hline PALLET ON THE FLOOR & 1984 & & Konsens \\
\hline
\end{tabular}

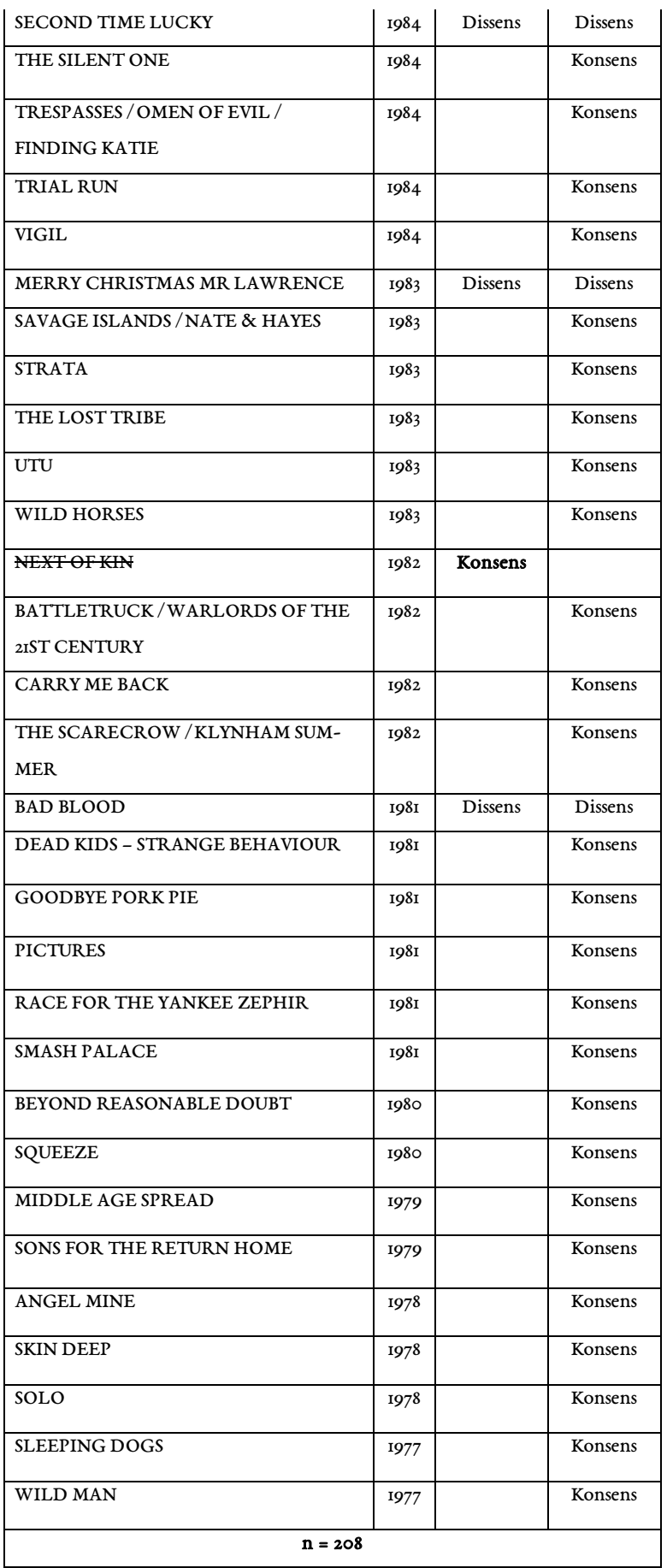

Tabelle 2.2: Konsens-Dissens-Analyse 
In der Tabelle sind in vier Spalten der Filmtitel, das Erscheinungsjahr, in der dritten Spalte das Ergebnis der Analyse, ob es zwischen den vier Filmkatalogen einen Konsens/Dissens darüber gibt, dass ein Film ausländisch ist, in der vierten Spalte das Ergebnis der Analyse, ob es zwischen den vier Katalogen einen Konsens/Dissens gibt, dass der betreffende Film als neuseeländisch einzustufen ist, eingetragen. Sofern sich die Verfasser aller Kataloge einig sind, dass ein Film ausländisch ist, beispielsweise durch den Vermerk „only locations“ oder „only associated with New Zealand“, dann ist dieser Film in der Tabelle durchgestrichen, da dieser Film aus dem zu untersuchenden Filmoeuvre ausgeschlossen wird. Aus logischen Gründen kann nur in einem der beiden Felder ein Konsens stehen, da beispielsweise bei einem Konsens über die „Ausländischkeit“ eines Films weder ein Dissens noch ein Konsens über dessen „Neuseeländischkeit" bestehen kann (ebenso umgekehrt). Sofern über einen Film ein Dissens herrscht, dass beispielsweise ein Filmkatalog jenen Film als ausländisch einstuft, wohingegen derselbe Film in einem anderen Filmkatalog als „Coproduktion“ aufgelistet ist, so besteht weder ein Konsens über die „Ausländischkeit“ noch über die „Neuseeländischkeit“ des Films, sodass in beiden Feldern ein Dissens stehen muss. Ist in einem Katalog ein Film nicht aufgeführt, so wird der Konsens bzw. Dissens aus den restlichen verfügbaren Katalogen ermittelt.

Ein voller Konsens über die „Neuseeländischkeit“ eines Films (dritte Spalte) wird als nicht notwendig erachtet, da bereits durch „neuseeländische“ Einstufung eines Films durch einen Katalog der Film autoritativ als neuseeländisch konstruiert wird und gerade die Ermittlung von Dissensen für die nachfolgende Untersuchung, inwiefern sich das Konzept des neuseeländischen Kinos verändert hat, relevant ist. Zudem kommt es bei Koproduktionen aufgrund ihrer „nationalen Hybridität“ auch auf die subjektive Betrachtungsweise des Filmkatalog-Erstellers an, je nachdem ob der Fokus mehr auf die neuseeländischen oder die ausländischen Anteile gerichtet ist, sodass zumindest in Grenzfällen oft ein Dissens zu erwarten ist, ob der Film noch als Koproduktion oder als ausländische Produktion einzustufen sei. Generell wird die Angabe „Coproduction“ in den Filmkatalogen als Aufnahmekriterium gewertet, da hiermit signalisiert wird, dass die Beteiligung Neuseelands an dem Film signifikant ist, sodass beispielsweise die Angabe „Coproduction“ in einem Filmkatalog und keinerlei Anmerkungen zu demselben Film in einem anderen Katalog (d. h. der Film wird dort als „rein neuseeländisch“ eingestuft) zu dem Eintrag „Konsens“ in der dritten Spalte führt. Umgekehrt wird ein Film, der beispielsweise nur in einem Filmkatalog als „ausländisch“ eingestuft wird, in das Filmoeuvre aufgenommen, da es keinen vollen Konsens gibt, dass der Film als „ausländisch“ 
bzw. „nicht-neuseeländisch“ einzustufen ist. Koproduktionen sind letztlich Hybride, die man einerseits als weder „rein national“, noch „rein ausländisch“, andererseits aber auch als sowohl „national“ als auch „ausländisch“ bewerten kann. In Zeiten verstärkter Globalisierung wäre ein Bestehen auf die „nationale Reinheit“ von Filmen unflexibel und nachteilhaft, da die Anzahl rein national produzierter Filme aufgrund der immer häufiger werdenden transnationalen Produktionsweisen weiter abnehmen dürfte.

Nach der Bereinigung von den Filmen, die im Konsens als „ausländisch“ eingestuft worden sind (II Filme), steht nun mit 197 Filmen das Filmoeuvre fest:

\begin{tabular}{|l|l|}
\hline \multicolumn{2}{|l|}{ Tabelle 2.3: Ermitteltes Filmkorpus } \\
\\
\hline A SONG OF GOOD & 2008 \\
\hline APRON STRINGS & 2008 \\
\hline DEAN SPANLEY & 2008 \\
\hline JINX SISTER & 2008 \\
\hline LAST OF THE LIVING & 2008 \\
\hline LOVE HAS NO LANGUAGE & 2008 \\
\hline SECOND-HAND WEDDING / GARAGE SALE & 2008 \\
\hline SHOW OF HANDS & 2008 \\
\hline THE MAP READER & 2006 \\
\hline 3O DAYS OF NIGHT & 2008 \\
\hline THE WATERHORSE: LEGEND OF THE DEEP & 2007 \\
\hline DOWN BY THE RIVERSIDE & 2007 \\
\hline EAGLE VS. SHARK & 2007 \\
\hline KISSY KISSY & 2006 \\
\hline MEN SHOULD'NT SING & 2007 \\
\hline THE DEVIL DARED ME TO & 2007 \\
\hline THE FERYMAN & 2007 \\
\hline THE LAST GREAT SNAIL CHASE & 2007 \\
\hline THE LAST MAGIC SHOW & 2007 \\
\hline THE TATTOOIST & 2007 \\
\hline WE'RE HERE TO HELP / BE VERY AFRAID & 2007 \\
\hline WHEN NIGHT FALLS & 2007 \\
\hline BLACK SHEEP & 2006 \\
\hline EVENT I6 & 2006 \\
\hline NO. 2 & 2006 \\
\hline OUT OF THE BLUE & \\
\hline PERFECT CREATURE & \\
\hline SIONE'S WEDDING & \\
\hline THE WAIMATE CONSPIRACY & \\
\hline
\end{tabular}

\begin{tabular}{|l|l|} 
WITHOUT A PADDLE & 2005 \\
\hline $\begin{array}{l}\text { THE CHRONICLES OF NARNIA: THE LION, } \\
\text { THE WITCH \& THE WARDROBE }\end{array}$ & 2005 \\
\hline SO WAYS OF SAYING FABULOUS & \\
\hline BOOGEYMAN & 2005 \\
\hline HIDDEN & 2005 \\
\hline KING KONG & 2005 \\
\hline LUELLA MILLER & 2005 \\
\hline MEET ME IN MIAMI & 2005 \\
\hline MEMORIES OF TOMORROW & 2005 \\
\hline QUIET NIGHT IN & 2005 \\
\hline RIVER QUEEN & 2005 \\
\hline THE WORLD'S FASTEST INDIAN & 2005 \\
\hline TYRANNICAL LOVE & 2005 \\
\hline INITE & 2005 \\
\hline FRACTURE /CRIME STORY & 2004 \\
\hline IN MY FATHER'S DEN & 2004 \\
\hline MURMURS & 2004 \\
\hline OFFENSIVE BEHAVIOUR & 2004 \\
\hline SPOOKED & 2004 \\
\hline CHRISTMAS & 2004 \\
\hline FOR GOOD & 2003 \\
\hline GUPTA VERSUS GORDON & 2003 \\
\hline I THINK I'M GOING & 2003 \\
\hline KOMBI NATION & 2003 \\
\hline $\begin{array}{l}\text { LORD OF THE RINGS: THE RETURN OF THE } \\
\text { KING }\end{array}$ & 2003 \\
\hline NEMESIS GAME / PAPER, SCISSORS, STONE & 2003 \\
\hline ORPHANS AND ANGELS & 2003 \\
\hline PERFECT STRANGERS & 2003 \\
\hline THE LAST SAMURAI & \\
\hline THE LOCALS & 2003 \\
\hline WHY CAN'T STOP THIS & \\
\hline UNCONTROLLABLE DANCING & \\
\hline
\end{tabular}




\begin{tabular}{|c|c|}
\hline WOODENHEAD & 2003 \\
\hline BLESSED & 2002 \\
\hline CUPID'S PREY & 2002 \\
\hline LORD OF THE RINGS: THE TWO TOWERS & 2002 \\
\hline OFF. & 2002 \\
\hline THE MĀORI MERCHANT OF VENICE & 2002 \\
\hline THIS IS NOT A LOVE STORY & 2002 \\
\hline TONGAN NINJA & 2002 \\
\hline TOY LOVE & 2002 \\
\hline WHALE RIDER & 2002 \\
\hline CROOKED EARTH & 2001 \\
\hline HER MAJESTY & 2001 \\
\hline KIDS WORLD & 2001 \\
\hline $\begin{array}{l}\text { LORD OF THE RINGS: FELLOWSHIP OF THE } \\
\text { RING }\end{array}$ & 2001 \\
\hline RAIN & $200 \mathrm{I}$ \\
\hline SNAKESKIN & 2001 \\
\hline STICKMEN & 2001 \\
\hline THE WAITING PLACE & 2001 \\
\hline $\begin{array}{l}\text { WHEN STRANGERS APPEAR / SHEARER'S } \\
\text { BREAKFAST }\end{array}$ & 2001 \\
\hline HOPELESS & 2000 \\
\hline JUBILEE & 2000 \\
\hline SAVAGE HONEYMOON & 2000 \\
\hline SHIFTER & 2000 \\
\hline THE FEATHERS OF PEACE & 2000 \\
\hline THE IRREFUTABLE TRUTH ABOUT DEMONS & 2000 \\
\hline THE PRICE OF MILK & 2000 \\
\hline WILD BLUE & 2000 \\
\hline CHANNELLING BABY & 1999 \\
\hline I'LL MAKE YOU HAPPY & 1999 \\
\hline MAGIK AND ROSE & 1999 \\
\hline SCARFIES / CRIME IOI & 1999 \\
\hline THE LUNATIC'S BALL & 1999 \\
\hline UNCOMFORTABLE COMFORTABLE & 1999 \\
\hline $\begin{array}{l}\text { WHAT BECOMES OF THE BROKEN } \\
\text { HEARTED? }\end{array}$ & 1999 \\
\hline MEMORY AND DESIRE & 1998 \\
\hline THE CLIMB & 1998 \\
\hline VIA SATELLITE & 1998 \\
\hline WHEN LOVE COMES & 1998 \\
\hline ABERRATION & 1997 \\
\hline SAVING GRACE & I997 \\
\hline THE UGLY & 1997 \\
\hline THE WHOLE OF THE MOON & 1997 \\
\hline $\begin{array}{l}\text { TOPLESS WOMEN TALK ABOUT THEIR } \\
\text { LIVES }\end{array}$ & 1997 \\
\hline
\end{tabular}

\begin{tabular}{|c|c|}
\hline BROKEN ENGLISH & 1996 \\
\hline CHICKEN & 1996 \\
\hline THE FRIGHTENERS & 1996 \\
\hline BONJOUR TIMOTHY & 1995 \\
\hline FLIGHT OF THE ALBATROSS & I995 \\
\hline $\begin{array}{l}\text { THE OFFERING / SHADOW OF THE PEPPER } \\
\text { TREE / ECLIPSE }\end{array}$ & I995 \\
\hline HEAVENLY CREATURES & 1994 \\
\hline JACK BROWN GENIUS & 1994 \\
\hline LOADED & 1994 \\
\hline ONCE WERE WARRIORS & 1994 \\
\hline THE LAST TATTOO & 1994 \\
\hline DESPERATE REMEDIES & 1993 \\
\hline JACK BE NIMBLE & 1993 \\
\hline THE PIANO & 1993 \\
\hline TRAFFIC ISLAND & 1993 \\
\hline ABSENT WITHOUT LEAVE & 1992 \\
\hline ALEX & 1992 \\
\hline BRAINDEAD & 1992 \\
\hline CHUNUK BAIR & 1992 \\
\hline CRUSH & 1992 \\
\hline $\begin{array}{l}\text { GRAMPIRE / MOONRISE / MY GRANDPA IS A } \\
\text { VAMPIRE }\end{array}$ & 1992 \\
\hline SECRETS / ONE CRAZY NIGHT & 1992 \\
\hline THE FOOTSTEP MAN & 1992 \\
\hline OLD SCORES & I99I \\
\hline TE RUA & 1991 \\
\hline THE END OF THE GOLDEN WEATHER & I991 \\
\hline AN ANGEL AT MY TABLE & 1990 \\
\hline MEET THE FEEBLES & 1990 \\
\hline RUBY AND RATA & 1990 \\
\hline THE RETURNING & 1990 \\
\hline USER FRIENDLY & 1990 \\
\hline CHILL FACTOR & 1989 \\
\hline FLYING FOX IN A FREEDOM TREE & 1989 \\
\hline ZILCH! & 1989 \\
\hline NEVER SAY DIE & I988 \\
\hline A SOLDIERS TALE & 1988 \\
\hline MAURI & 1988 \\
\hline SEND A GORILLA & I988 \\
\hline THE NAVIGATOR & I988 \\
\hline WILLOW & 1988 \\
\hline BAD TASTE & I987 \\
\hline FOOTROT FLATS & 1987 \\
\hline ILLUSTRIOUS ENERGY & 1987 \\
\hline NGATI & 1987 \\
\hline STARLIGHT HOTEL & 1987 \\
\hline ARRIVING TUESDAY & 1986 \\
\hline
\end{tabular}




\begin{tabular}{|l|l|} 
BRIDGE TO NOWHERE & $\mathbf{1 9 8 6}$ \\
\hline MESMERIZED / SHOCKED & $\mathbf{1 9 8 6}$ \\
\hline QUEEN CITY ROCKER & $\mathbf{1 9 8 6}$ \\
\hline CAME A HOT FRIDAY & $\mathbf{1 9 8 5}$ \\
\hline DANGEROUS ORPHANS & $\mathbf{1 9 8 5}$ \\
\hline KINGPIN & $\mathbf{1 9 8 5}$ \\
\hline LEAVE ALL FAIR & $\mathbf{1 9 8 5}$ \\
\hline MR WRONG / DARK OF THE NIGHT & $\mathbf{1 9 8 5}$ \\
\hline SHAKER RUN & $\mathbf{1 9 8 5}$ \\
\hline SHOULD I BE GOOD? & $\mathbf{1 9 8 5}$ \\
\hline SYLVIA & $\mathbf{1 9 8 5}$ \\
\hline THE QUIET EARTH & $\mathbf{1 9 8 5}$ \\
\hline THE BOUNTY & $\mathbf{1 9 8 4}$ \\
\hline CONSTANCE & $\mathbf{1 9 8 4}$ \\
\hline DEATH WARMED UP & $\mathbf{1 9 8 4}$ \\
\hline HEART OF THE STAG & $\mathbf{1 9 8 4}$ \\
\hline OTHER HALVES & $\mathbf{1 9 8 4}$ \\
\hline PALLET ON THE FLOOR & $\mathbf{1 9 8 4}$ \\
\hline SECOND TIME LUCKY & $\mathbf{1 9 8 4}$ \\
\hline THE SILENT ONE & $\mathbf{1 9 8 4}$ \\
\hline TRESPASSES / OMEN OF EVIL /FINDING & $\mathbf{1 9 8 4}$ \\
\hline KATIE & $\mathbf{1 9 8 3}$ \\
\hline TRIAL RUN & $\mathbf{1 9 8 4}$ \\
\hline VIGIL & $\mathbf{1 9 8 4}$ \\
\hline MERRY CHRISTMAS MR LAWRENCE & $\mathbf{1 9 8 3}$ \\
\hline SAVAGE ISLANDS /NATE \& HAYES & \\
\hline STRATA & \\
\hline
\end{tabular}

\begin{tabular}{|c|c|}
\hline THE LOST TRIBE & 1983 \\
\hline UTU & 1983 \\
\hline WILD HORSES & 1983 \\
\hline $\begin{array}{l}\text { BATTLETRUCK / WARLORDS OF THE 2IST } \\
\text { CENTURY }\end{array}$ & 1982 \\
\hline CARRY ME BACK & 1982 \\
\hline THE SCARECROW / KLYNHAM SUMMER & 1982 \\
\hline BAD BLOOD & I98I \\
\hline DEAD KIDS - STRANGE BEHAVIOUR & I98I \\
\hline GOODBYE PORK PIE & 1981 \\
\hline PICTURES & I98I \\
\hline RACE FOR THE YANKEE ZEPHIR & I98I \\
\hline SMASH PALACE & I98I \\
\hline BEYOND REASONABLE DOUBT & 1980 \\
\hline SQUEEZE & 1980 \\
\hline MIDDLE AGE SPREAD & 1979 \\
\hline $\begin{array}{l}\text { SONS FOR THE RETURN HOME (DIE SÖHNE } \\
\text { KEHREN ZURÜCK) }\end{array}$ & 1979 \\
\hline ANGEL MINE & 1978 \\
\hline SKIN DEEP & 1978 \\
\hline SOLO & 1978 \\
\hline SLEEPING DOGS & 1977 \\
\hline WILD MAN & 1977 \\
\hline Gesamtanzahl Filme $n=$ & 197 \\
\hline
\end{tabular}

Das ermittelte Filmoeuvre sowie die Konsens-Dissens-Analyse bilden die Grundlage für alle nachfolgenden Analysen. Wichtig ist hier, dass dieses Filmoeuvre zwar die Analysegrundlage bildet, aber nicht etwa eine „definitivere“, essenzialistische Version des nationalen neuseeländischen Kinos darstellen soll, die aus den zuvor genannten Filmkatalogen „destilliert“ worden ist. Stattdessen soll gerade durch die explizite Einbeziehung von Filmen, über die ein Dissens besteht, klargestellt werden, dass es keinen generellen Konsens über ein nationales neuseeländisches Kino gibt.

\subsection{Analyse}

Anhand einer grafischen Aufbereitung der Ergebnisse der Konsens-Dissens-Analyse soll nun die eingangs erwähnte Frage näher erörtert werden, was für Auswirkungen etwaige Verände- 
rungen der neuseeländischen Filmindustrie auf das Konzept bzw. auf die Konstruktion des „nationalen neuseeländischen Kinos“ gehabt haben könnten:

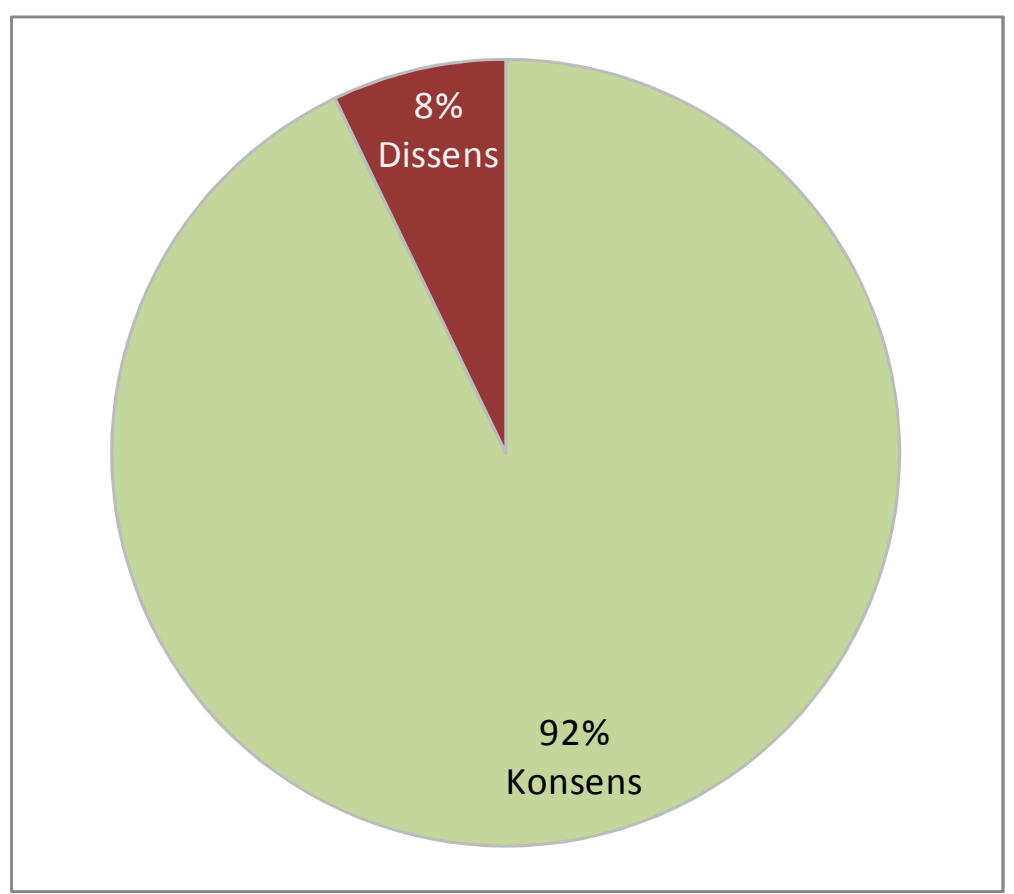

Abbildung 2.I: Konsens/Dissens, ob ein Film neuseeländisch ist

In Abbildung 2.I ist mittels eines Tortendiagramms dargestellt, wie hoch der Konsens unter den vier Filmkatalogen ist, dass die Filme des ermittelten Filmoeuvres neuseeländisch sind (entspricht Spalte 4 in Tabelle 2.2). Der Konsens ist mit $92 \%$ sehr hoch. Allerdings ist ein Dissens von rund $8 \%$ ein deutlicher Wert, ${ }^{22}$ der erkennen lässt, dass sich aufgrund ausländischer Einflüsse auf die neuseeländische Filmindustrie das Konzept des „nationalen neuseeländischen Kinos“ destabilisiert hat und einen vollen Konsens nicht zulässt. Aufgrund des Konstruktcharakters dieses Konzepts kann zwar nicht von „Zuordnungsfehlern“ seitens der Katalog-Ersteller gesprochen werden, da eine subjektive Filmzuordnung letztendlich weder „richtig“ noch „falsch“ sein kann. Es zeigt sich aber, dass über $8 \%$ der Filme keine Einigkeit herrscht, ob sie noch als neuseeländische oder aber bereits als ausländische Produktionen zu werten sind. Dies könnte als „subjektive Denationalisierung“ bezeichnet werden, da der Prozess der Denationalisierung ebenfalls Teil des Konstrukts des neuseeländischen Kinos ist und daher nicht rein objektiv messbar, sondern zumindest in Teilen subjektiver Natur ist und von den normativ gesetzten Rahmenbedingungen abhängig ist. So sind die

\footnotetext{
${ }^{22}$ Wert wurde aufgerundet
} 
Filmkataloge Produkt der von diversen normativen und zum Teil rein subjektiven Kriterien geprägten Filmauswahl der Filmkatalog-Ersteller, was einen direkten Einfluss auf das Analyseergebnis bezüglich „Denationalisierung“ und „Renationalisierung“ hat.

Da Abbildung 2.I nur einen statischen Zustand abbildet, soll nun der zeitliche Verlauf untersucht werden, woraus ersichtlich wird, wann die subjektive Denationalisierung begonnen hat und ob sich ein Trend erkennen lässt:

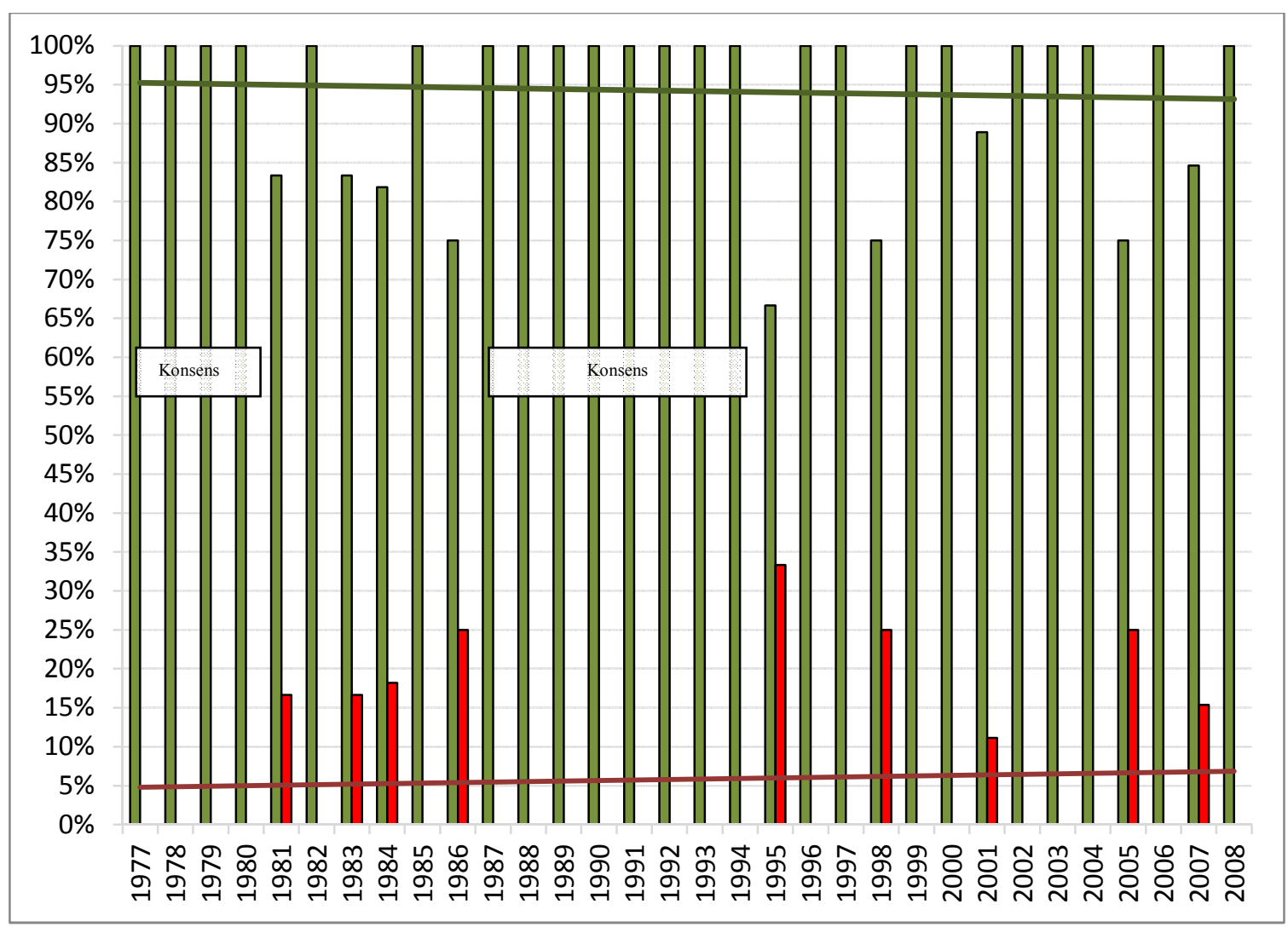

Abbildung 2.2: Konsens/Dissens - zeitlicher Verlauf

Abbildung 2.2 zeigt auf der X-Achse die Jahreszahlen, über der alle Filme, die im entsprechenden Jahr ausgestrahlt wurden, in einem bzw. zwei Balken zusammengefasst dargestellt sind. Die Y-Achse zeigt den relativen Anteil der Filme, über die ein Konsens (grün) oder ein Dissens (rot) herrscht. Sofern in einem Jahr ein grüner und ein roter Balken zu sehen sind, ist die Summe der beiden Balken immer Ioo \%.

Das Diagramm zeigt, dass für die Anfangsjahre des zeitgenössischen neuseeländischen Kinos von 1977 bis 1980 ein $100 \%$ iger Konsens herrscht, d. h. dass Ioo \% der in diesen Jahren im Filmoeuvre angeführten Filme als neuseeländisch eingestuft werden. Für das Jahr I98I gibt es erstmals 
einen Dissens über I6,6\% der Filme. Konkret bedeutet dies, dass fünf der sechs Filme, die in Tabelle 2.I für 198I aufgelistet sind, geschlossen als neuseeländisch eingestuft werden, wohingegen der sechste Film Bad Blood unterschiedlich eingestuft wird: Von der IMDb, von Conrich und von Martin wird der Film als Koproduktion eingestuft, während die NZFC ihn als ausländisch einstuft („only locations“) - und das, obwohl der Film inhaltlich zweifelsfrei ein neuseeländisches Thema behandelt. Die Uneinigkeit lässt sich an dem ausgesprochen „national hybriden“ Charakter des Films erklären: Während die Schauspieler vorwiegend neuseeländisch sind und in Neuseeland gedreht wurde, wurde der Film allein von der britischen Firma Southern Pictures produziert, unter Regie von dem Engländer Mike Newell. Hinsichtlich des Drehbuchautors Andrew Brown kommt es darauf an, ob auf seinen Geburtsort Neuseeland abgestellt wird oder auf seinen späteren Wohnort in Australien. Ähnlich verhält es sich beispielsweise auch im Falle Jane Campions, einer Australierin, die in Neuseeland geboren wurde. So weist Rochelle Simmons in ihrem Aufsatz „The Piano" darauf hin, dass Campion weiterhin sowohl mit Neuseeland als auch mit Australien assoziiert wird (2007: 149), obwohl sie während des Drehs von The Piano bereits lange Zeit die australische Staatsbürgerschaft innehatte und damit Teil der australischen und nicht der neuseeländischen Ökonomie ist. Dies dürfte damit zusammenhängen, dass eine derart renommierte Regisseurin aus Prestigegründen von Neuseeland „vereinnahmt“ wird, um die nationale Filmindustrie in ein positives Licht zu rücken. Dahinterliegend sind sowohl eine generelle nationale Agenda als auch konkrete Marketingstrategien zu erblicken.

Dass es im Zeitraum 198I-1986 relativ viele Filme gibt, die einen Dissens bilden, lässt sich vor allem vor dem Hintergrund der besonderen Steuergesetze zu dieser Zeit erklären. Anfang der 8oer Jahre wurden steuerliche Bestimmungen nach australischem Vorbild in Neuseeland eingeführt, die es Filmemachern erlaubten, teilweise über I50 \% ihrer Investitionen steuerlich abzusetzen, was auch viele ausländische Investoren anlockte (Roddick, 2008: 37). Hierdurch nahm die Anzahl in Neuseeland (ko-)produzierter Filme dramatisch zu, was als Renationalisierung bezeichnet werden kann, da sich die „Stärke“ eines nationalen Kinos auch an der Größe des Filmoeuvres bestimmt, d. h. dass nicht nur qualitative, sondern auch quantitative Aspekte entscheidend sind. Durch die verstärkte Involvierung ausländischer Investoren und Filmmitarbeiter sind diese Filme wie schon Bad Blood „nationale Hybride“, die einen verstärkten Dissens bezüglich ihrer Einordnung in den Katalogen hervorrufen. 
Nachdem die steuerlichen Vorteile immer exzessiver ausgenutzt wurden, sah sich die neuseeländische Regierung veranlasst, das Steuerloch Ende 1984 zu schließen (Roddick, 2008: 37), weshalb es rund ein Jahr später unweigerlich zu einem Einbruch der Filmanzahl kam (Abbildung 2.3).

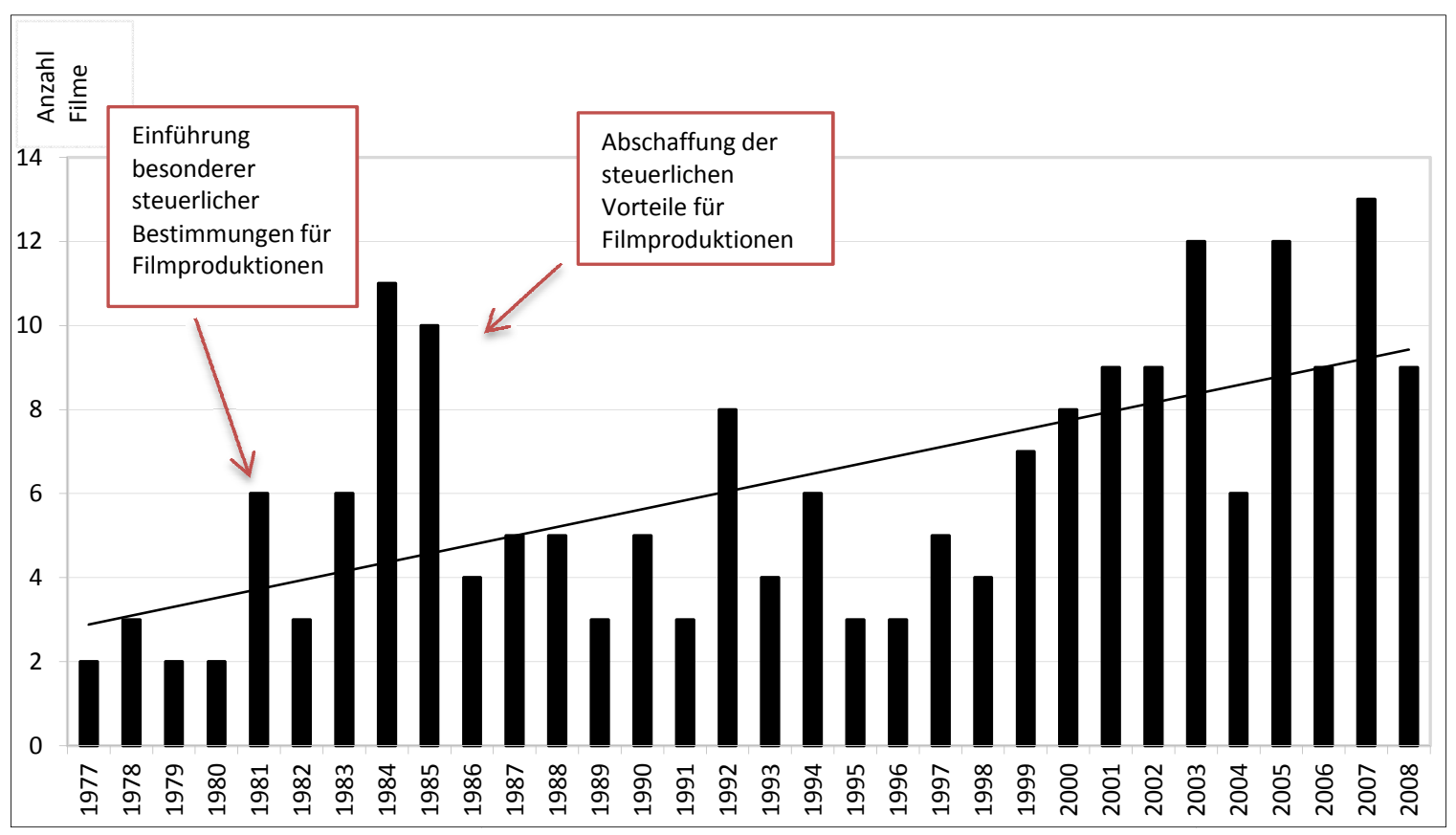

Abbildung 2.3: Entwicklung Anzahl neuseeländische Filme, 1977-2008

Da das ökonomische Klima für Filmemacher nun deutlich rauer wurde, wurde viel ausländisches Kapital abgezogen, was dazu führte, dass die produzierten Filme aus nationaler Sicht wieder „reiner“ wurden - was auch an dem stabilen Konsens im Zeitraum 1987-1994 zu erkennen ist (Abbildung 2.2). Folglich kann das neuseeländische Kino zu dieser Zeit als renationalisiert bezeichnet werden. Während also die einzelnen Filme insofern als renationalisiert angesehen werden können, hat sich in diesem Zeitraum durch die im Mittel gesunkene Filmanzahl das neuseeländische Kino aber auch denationalisiert. Erst im Jahr 1995 kommt es wieder zu einem deutlichen Dissens, um dann in den folgenden Jahren in relativ regelmäßigen Abständen wiederzukehren, allerdings in größeren zeitlichen Abständen als zu Zeiten der „forcierten“ De- bzw. Renationalisierung durch die Steuergesetze 198I-1984 (Abbildung 2.2). Dass es ab 1995 wieder vermehrt zu Dissensen kommt, lässt sich an den Erfolgen von Filmen wie The Piano (1993), Once Were Warriors (1994), Whale Rider (2002) und der Lord of the Rings-Trilogie (200I-2003) erklären, wodurch wieder verstärkt ausländische Investoren angelockt wurden und dadurch die „nationale Reinheit“ der Filme sich wieder verringert hat. 
In Gesamtbetrachtung zeigt die Trendlinie (rot) für den Dissens in Abbildung 2.2 einen sehr leicht ansteigenden Trend von rund $2 \%$ an (was umgekehrt einer Abnahme des Konsenses um $2 \%$ entspricht [grüne Trendlinie]), wohingegen die Trendlinie in Abbildung 2.3 einen deutlichen Anstieg von durchschnittlich 3 auf 9 Filme pro Jahr anzeigt, was einer Steigerung von $300 \%$ entspricht. Somit steht einer sehr leichten subjektiven Denationalisierung auf qualitativer Ebene (Einstufung der Zusammensetzung der einzelnen Filme in den Filmkatalogen) eine sehr starke Renationalisierung auf quantitativer Ebene (Filmanzahl) gegenüber. Es wird auch erkennbar, dass im neuseeländischen Kino die Zunahme der Anzahl der produzierten Filme regelmäßig mit der Verringerung der (subjektiv empfundenen) „nationalen Homogenität“ der einzelnen Filme korreliert. Mit anderen Worten führt aus Sicht der Filmkatalogersteller die quantitative Zunahme des Filmoutputs zu einer gleichzeitigen Abnahme der „nationalen Qualität“. Im Endeffekt bedeutet dies, dass die wirtschaftlichen Interessen, d. h. hoher Filmoutput, gefördert durch diverse „Grants“, wie z. B. dem Large Budget Screen Production Grant, in Konkurrenz zu der nationalen Agenda der New Zealand Film Commission treten, deren explizite Aufgabe es ist, Filme mit „significant New Zealand content“ zu fördern („New Zealand Film Commission Act 1978“: 8), d. h. Filme, die sich durch eine besondere „nationale Spezifizität“ auszeichnen - und dies nicht nur inhaltlich, sondern auch in puncto Produktionsbedingungen.

Nun stellt sich die Frage, wie der qualitative Aspekt der Filme, d. h. deren Zusammensetzung aus Produktionssicht, von den einzelnen Filmkatalogen bewertet wird und welche Kriterien und Kriterienkonstellationen tatsächlich ausschlaggebend sind, damit ein Film als „neuseeländisch“ oder „ausländisch“ eingestuft wird. Zur Ermittlung wurde eine Kriterien-Matrix angelegt, die im Folgenden dargestellt ist: 
Tabelle 2.4: Kriterien-Matrix

\begin{tabular}{|c|c|c|c|c|c|c|c|c|c|c|}
\hline \multicolumn{4}{|c|}{ Kriterienkonstellation } & \multirow[t]{2}{*}{ Film } & \multirow[b]{2}{*}{ 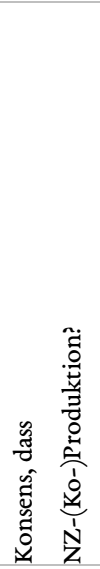 } & \multirow[b]{2}{*}{ 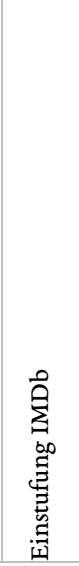 } & \multirow[b]{2}{*}{ 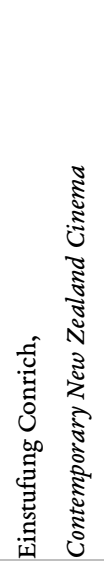 } & \multirow[b]{2}{*}{ 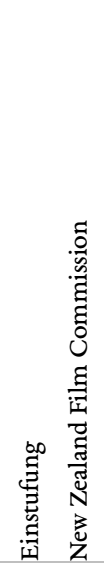 } & \multirow[b]{2}{*}{ 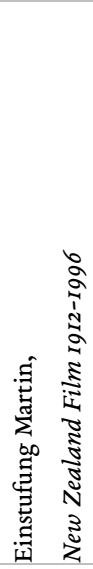 } & \multirow[b]{2}{*}{ 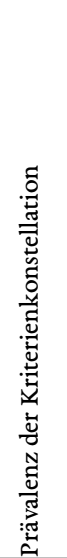 } \\
\hline 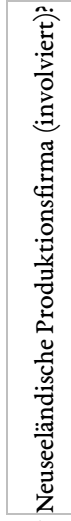 & 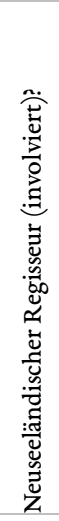 & 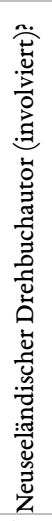 & 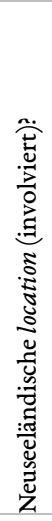 & & & & & & & \\
\hline \multicolumn{11}{|c|}{ Einer-Konstellation } \\
\hline \multirow[t]{8}{*}{-} & - & - & + & 30 DAYS OF NIGHT (2007) & nein & Kp. ${ }^{\prime}$ & Kp. & ausl.* & n. v.* & $4, \mathrm{r} \%$ \\
\hline & & & & WATERHORSE (2007) & nein & Kp. & ausl. & Kp. & n.v. & \\
\hline & & & & WITHOUT A PADDLE (2005) & nein & Kp. & ausl. & ausl. & n.v. & \\
\hline & & & & LAST SAMURAI (2003) & nein & Kp. & ausl. & ausl. & n.v. & \\
\hline & & & & HER MAJESTY (200I) & nein & $\mathrm{NZ}$ & Kp. & ausl. & n.v. & \\
\hline & & & & THE PIANO (1993) & ja & Кр. & Kp. & $\mathrm{NZ}$ & Kp. & \\
\hline & & & & WILLOW (rg89) & nein & Kp. & ausl. & ausl. & ausl. & \\
\hline & & & & MESMERIZED (1986) & nein & Kр. & Kp. & ausl. & Kp. & \\
\hline
\end{tabular}

Ergebnis: Dissens

Zweier-Konstellationen

\begin{tabular}{|c|c|c|c|c|c|c|c|c|c|c|}
\hline+ & - & - & + & $\begin{array}{l}\text { MEET ME IN MIAMI (2005) } \\
\text { WORLD'S FASTEST INDIAN } \\
(2005) \\
\text { HIDDEN (2005) } \\
\text { TOY LOVE (2002) } \\
\text { PRICE OF MILK (2000) } \\
\text { WILD BLUE (2000) } \\
\text { CLIMB (1998) } \\
\text { TOPLESS WOMEN (1997) } \\
\text { LOADED (1994) } \\
\text { SECRETS (1992) } \\
\text { CHILL FACTOR (1989) } \\
\text { MERRY CHRISTMAS LAW- } \\
\text { RENCE (1983) } \\
\text { DEAD KIDS (198I) } \\
\text { RACE FOR THE YANKEE } \\
\text { ZEPHYR (198I) }\end{array}$ & $\begin{array}{c}\text { nein } \\
\text { ja } \\
\text { ja } \\
\text { ja } \\
\text { ja } \\
\text { ja } \\
\text { nein } \\
\text { ja } \\
\text { ja } \\
\text { ja } \\
\text { ja } \\
\text { nein } \\
\text { ja } \\
\text { ja }\end{array}$ & $\begin{array}{l}\text { NZ } \\
\text { Kp. } \\
\text { NZ } \\
\text { NZ } \\
\text { NZ } \\
\text { NZ } \\
\text { Kp. } \\
\text { NZ } \\
\text { Kp. } \\
\text { Kp. } \\
\text { NZ } \\
\text { Kp. } \\
\text { Kp. } \\
\text { Kp. }\end{array}$ & $\begin{array}{l}\text { NZ } \\
\text { NZ } \\
\text { NZ } \\
\text { NZ } \\
\text { Kp. } \\
\text { NZ } \\
\text { Kp. } \\
\text { Kp. } \\
\text { Kp. } \\
\text { Kp. }\end{array}$ & $\begin{array}{c}\text { n. v. } \\
\mathrm{NZ} \\
\mathrm{NZ} \\
\mathrm{NZ} \\
\text { ausl. } \\
\mathrm{NZ} \\
\mathrm{NZ} \\
\mathrm{NZ} \\
\mathrm{NZ} \\
\text { ausl. } \\
\mathrm{NZ} \\
\mathrm{NZ}\end{array}$ & $\begin{array}{l}\text { n. v. } \\
\text { n. v. } \\
\text { n. v. } \\
\text { n. v. } \\
\text { n. v. } \\
\text { n. v. } \\
\text { Kp. } \\
\text { Kp. } \\
\text { Kp. } \\
\text { Kp. }\end{array}$ & $7, \mathrm{I} \%$ \\
\hline \multicolumn{11}{|c|}{ Ergebnis: } \\
\hline - & + & - & + & THE BOUNTY (1984) & nein & Kp. & Kp. & ausl. & ausl. & $0,5 \%$ \\
\hline \multicolumn{11}{|c|}{ Ergebnis: (Dissens) } \\
\hline - & - & + & + & BAD BLOOD (r98I) & nein & Kp. & Kp. & ausl. & Kp. & $0,5 \%$ \\
\hline
\end{tabular}


Dreier-Konstellationen

\begin{tabular}{|c|c|c|c|c|c|c|c|c|c|c|}
\hline - & + & + & + & $\begin{array}{l}\text { THE CHRONICLES OF } \\
\text { NARNIA I (2005) } \\
\text { SQUEEZE (1980) }\end{array}$ & ja & $\begin{array}{l}\text { ausl. } \\
\text { NZ }\end{array}$ & $\begin{array}{l}\text { ausl. } \\
\text { NZ }\end{array}$ & $\mathrm{NZ}$ & $\mathrm{NZ}$ & $1,0 \%$ \\
\hline \multicolumn{11}{|c|}{ Ergebnis: (Dissens) } \\
\hline+ & - & + & + & $\begin{array}{l}\text { CUPIDS PREY (2002) } \\
\text { KIDS WORLD (200I) } \\
\text { ABERRATION (1997) } \\
\text { FLIGHT OF ALBATROSS } \\
\text { (1995) } \\
\text { TRAFFIC ISLAND (1993) } \\
\text { ALEX (1992) } \\
\text { CHUNUK BAIR (1992) } \\
\text { SECOND TIME LUCKY (1984) } \\
\text { SAVAGE ISLANDS (1983) } \\
\text { BATTLETRUCK (1982) }\end{array}$ & $\begin{array}{c}\text { ja } \\
\text { ja } \\
\text { ja } \\
\text { nein } \\
\text { ja } \\
\text { ja } \\
\text { ja } \\
\text { nein } \\
\text { ja } \\
\text { ja }\end{array}$ & $\begin{array}{l}\text { NZ } \\
\text { Kp. } \\
\text { NZ } \\
\text { NZ } \\
\text { Kp. } \\
\text { NZ }\end{array}$ & $\begin{array}{l}\text { NZ } \\
\text { NZ } \\
\text { NZ } \\
\text { Kp. } \\
\text { NZ } \\
\text { NZ } \\
\text { NZ } \\
\text { Kp. } \\
\text { NZ } \\
\text { NZ }\end{array}$ & $\begin{array}{l}\text { n. v. } \\
\text { NZ } \\
\text { NZ } \\
\text { ausl. } \\
\text { NZ } \\
\text { NZ }\end{array}$ & $\begin{array}{l}\text { n. v. } \\
\text { Kp. } \\
\text { NZ } \\
\text { Kp. } \\
\text { Kp. } \\
\text { Kp. }\end{array}$ & $5,1 \%$ \\
\hline \multicolumn{11}{|c|}{ Ergebnis: } \\
\hline+ & + & - & + & $\begin{array}{l}\text { DEAN SPANLEY (2008) } \\
\text { BOOGEYMAN (2005) }\end{array}$ & $\begin{array}{c}\text { ja } \\
\text { nein }\end{array}$ & $\begin{array}{l}\text { Kp. } \\
\text { Kp. }\end{array}$ & $\begin{array}{l}\text { Kp. } \\
\text { ausl. }\end{array}$ & $\begin{array}{l}\text { Kp. } \\
\text { NZ }\end{array}$ & $\begin{array}{l}\text { n. v. } \\
\text { n. v. }\end{array}$ & $1,0 \%$ \\
\hline \multicolumn{11}{|c|}{ Ergebnis: (Dissens) } \\
\hline+ & + & + & - & $\begin{array}{l}\text { THE OFFERING (1995) } \\
\text { FLYING FOX IN FREEDOM } \\
\text { TREE (1989) } \\
\text { A SOLDIERS TALE (1988) } \\
\text { LEAVE ALL FAIR (1985) } \\
\text { THE SILENT ONE (1984) }\end{array}$ & $\begin{array}{l}\text { ja } \\
\text { ja } \\
\text { ja }\end{array}$ & $\begin{array}{l}\text { NZ } \\
\text { Kp. } \\
\text { NZ }\end{array}$ & $\begin{array}{l}\mathrm{NZ} \\
\mathrm{NZ} \\
\mathrm{NZ}\end{array}$ & $\begin{array}{l}\mathrm{NZ} \\
\mathrm{NZ} \\
\mathrm{NZ}\end{array}$ & $\begin{array}{l}\text { NZ } \\
\text { Kp. } \\
\text { NZ }\end{array}$ & $2,5 \%$ \\
\hline
\end{tabular}

Ergebnis: (Konsens)

Vierer-Konstellation

\begin{tabular}{|c|c|c|c|c|c|c|c|c|c|c|}
\hline+ & + & + & + & $\begin{array}{l}\text { A SONG OF GOOD (2008) } \\
\text { APRON STRINGS (2008) } \\
\text { JINX SISTER (2008) } \\
\text { LAST OF THE LIVING (2008) } \\
\text { SECOND-HAND WEDDING } \\
\text { (2008) } \\
\text { SHOW OF HANDS (2008) } \\
\text { THE MAP READER (2008) } \\
\text { LOVE HAS NO LANGUAGE } \\
\text { (2008) } \\
\text { EAGLE VS. SHARK (2007) } \\
\text { KISSY KISSY (2007) } \\
\text { THE DEVIL DARED ME TO } \\
\text { (2007) } \\
\text { THE FERYMAN (2007) } \\
\text { THE LAST MAGIC SHOW } \\
\text { (2007) }\end{array}$ & $\begin{array}{l}\text { ja } \\
\text { ja } \\
\text { ja } \\
\text { ja } \\
\text { ja } \\
\text { ja } \\
\text { ja } \\
\text { ja } \\
\text { ja } \\
\text { ja }\end{array}$ & $\begin{array}{l}\mathrm{NZ} \\
\mathrm{NZ} \\
\mathrm{NZ} \\
\mathrm{NZ} \\
\mathrm{NZ} \\
\mathrm{NZ} \\
\mathrm{NZ} \\
\mathrm{Kp} . \\
\mathrm{NZ} \\
\mathrm{NZ} \\
\mathrm{NZ} \\
\mathrm{Kp} . \\
\mathrm{NZ}\end{array}$ & $\begin{array}{l}\text { NZ } \\
\text { NZ } \\
\text { NZ } \\
\text { NZ } \\
\text { NZ } \\
\text { NZ } \\
\text { n. v. } \\
\text { n. v. } \\
\text { NZ } \\
\text { NZ } \\
\text { NZ } \\
\text { Kp. } \\
\text { NZ }\end{array}$ & $\begin{array}{l}\text { NZ } \\
\text { NZ } \\
\text { NZ } \\
\text { NZ } \\
\text { NZ } \\
\text { NZ } \\
\text { NZ } \\
\text { Kp. } \\
\text { NZ } \\
\text { NZ } \\
\text { NZ } \\
\text { NZ } \\
\text { NZ }\end{array}$ & $\begin{array}{l}\text { n. v. } \\
\text { n. v. } \\
\text { n. v. } \\
\text { n. v. } \\
\text { n. v. }\end{array}$ & $78 \%$ \\
\hline
\end{tabular}




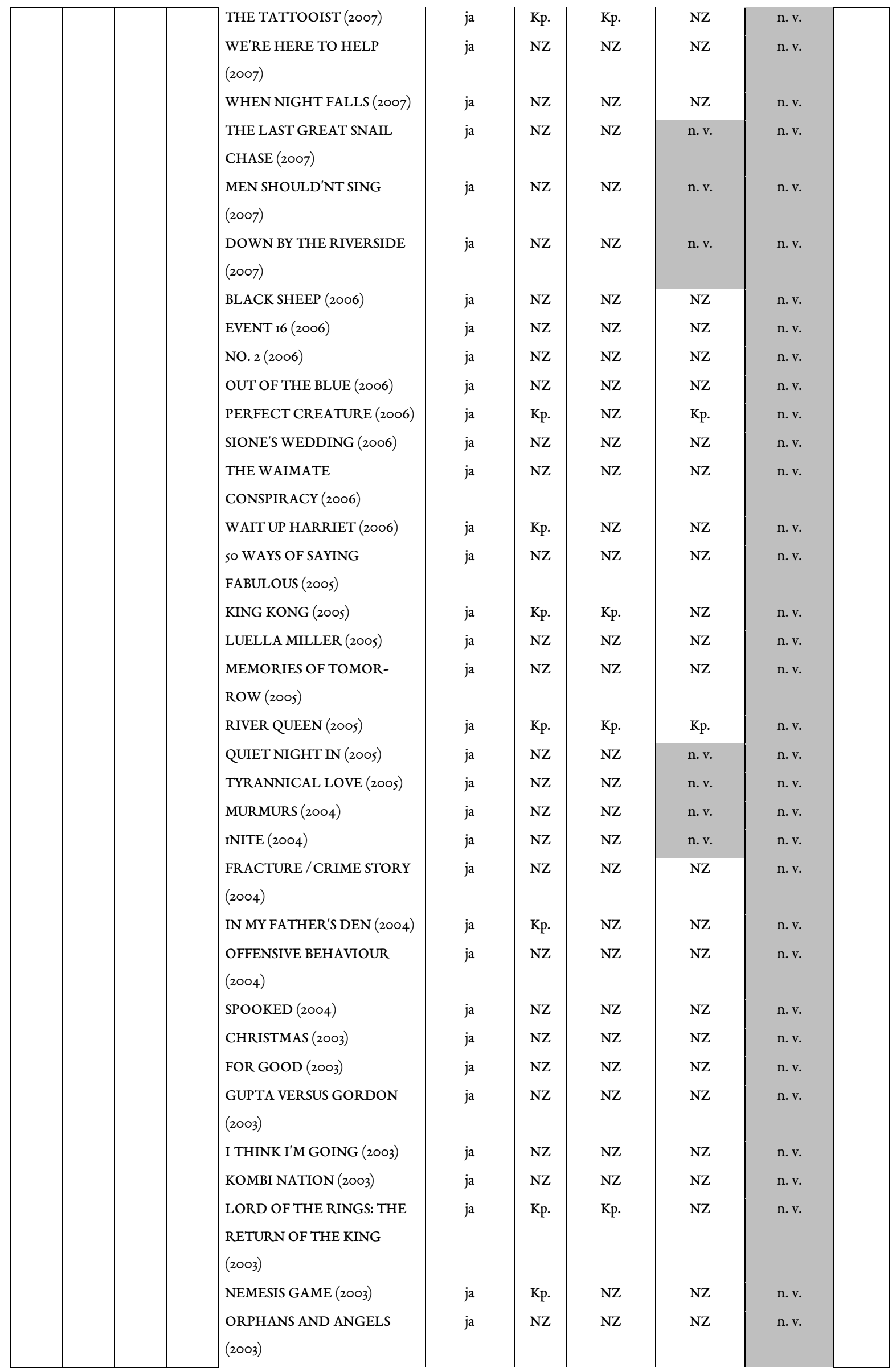




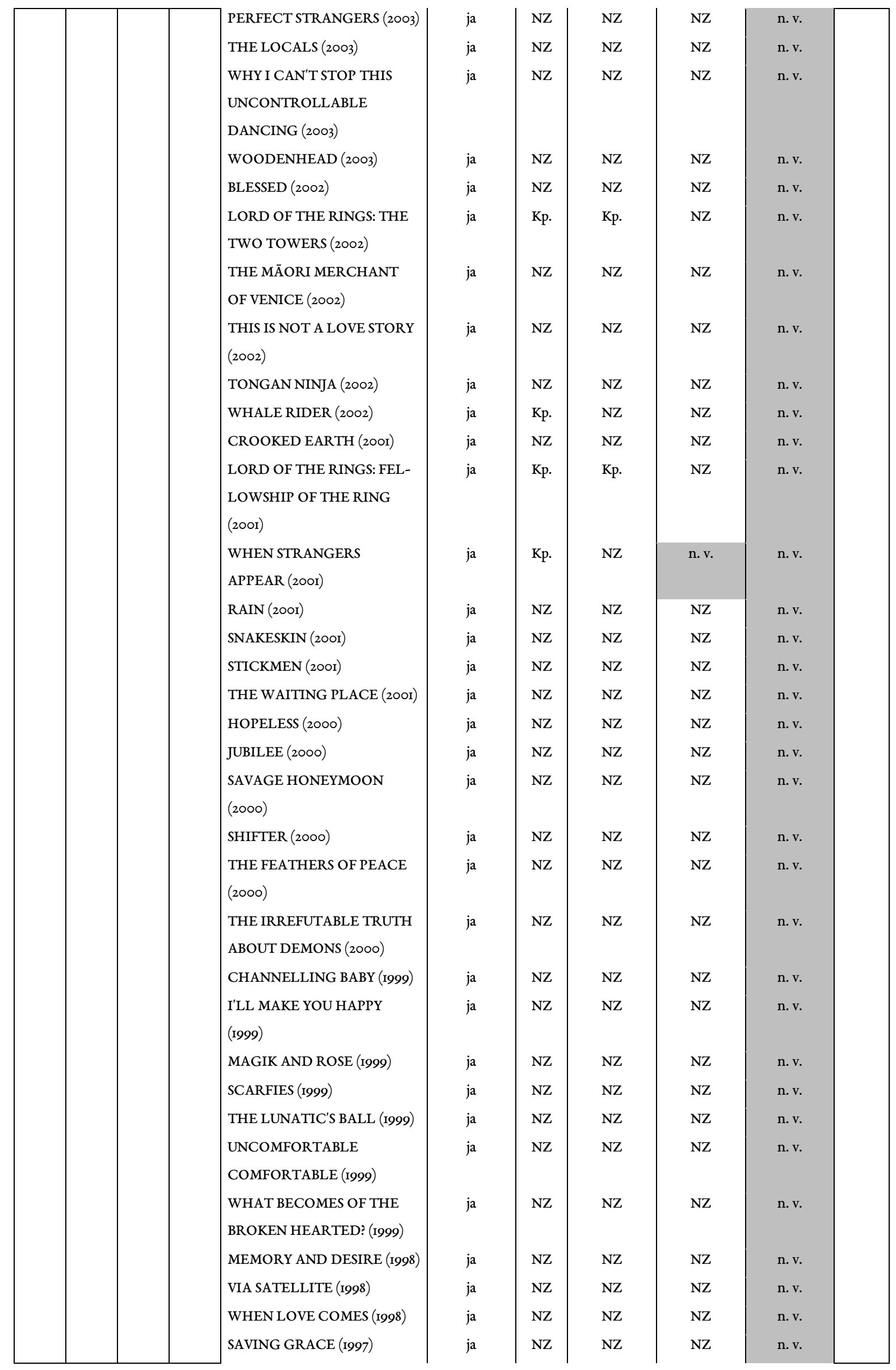




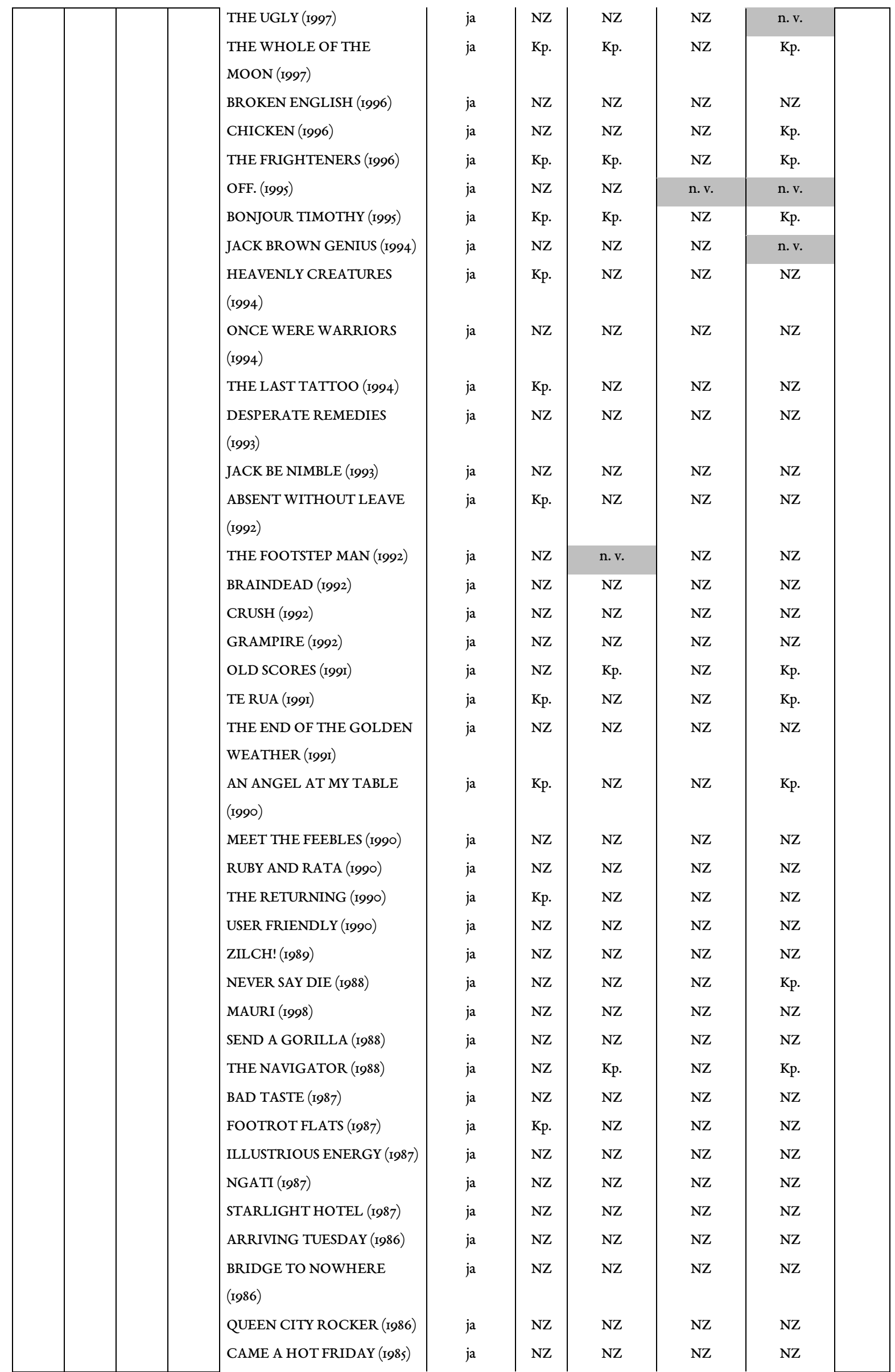




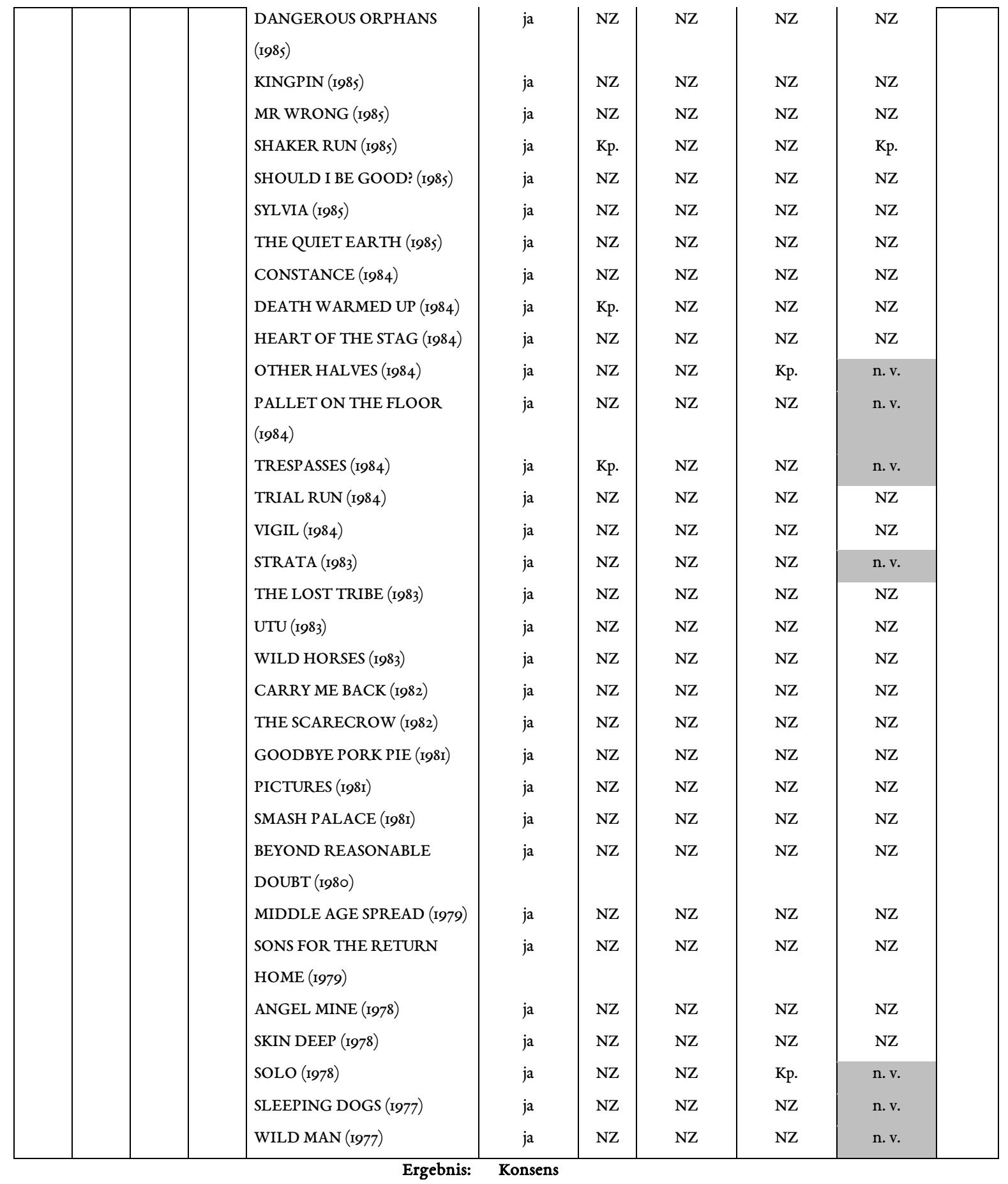

*) Kp. = Koproduktion; ausl. = ausländisch; n. v. = nicht verfügbar (keine Daten); NZ = neuseeländisch

Dargestellt sind in den vier linken Spalten die untersuchten Kriterien bzw.

Kriterienkonstellationen (Nationalitäten der Produktionsfirma, des Regisseurs, des Drehbuchautors und der location). Der Zusatz „involviert“ in der Spaltenbetitelung signalisiert, dass auch Koproduktionen zwischen ausländischen und neuseeländischen Produktionsfirmen sowie die Zusammenarbeit zwischen neuseeländischen und ausländischen Regisseuren und Drehbuchautoren 
als auch die Verwendung neuseeländischer zusammen mit ausländischen locations als „neuseeländisch“ gewertet werden. Ein „+“ signalisiert „neuseeländisch“ / „neuseeländisch-ausländisch“, d.h. eine Koproduktion, wohingegen ein „-“ „,rein ausländisch“ bedeutet. Kriterienkonstellationen, die in der Tabelle nicht angeführt sind, kommen im analysierten Filmoeuvre nicht vor. Neben der Filmtitelspalte ist die Konsens-Dissens-Auswertung bezüglich der verschiedenen Kriterienkonstellationen zu sehen. Sofern dort nicht für jeden Film der entsprechenden Kriterienkonstellation ein ,ja“ für Konsens über die „Neuseeländischkeit“ des Films angegeben ist, wird dies im Ergebnis als Dissens gewertet. Nur bei vollem Konsens über alle Filme, die einer entsprechenden Kriterienkonstellation angehören, ist das Gesamtergebnis ein Konsens. Neben der Konsens-Dissens-Spalte sind die einzelnen Einstufungen der vier Filmkataloge aufgelistet, wobei die horizontale Summierung der Einzelwertungen das (Zwischen-)Ergebnis für die Konsens-Dissens-Feststellung ergibt und außerdem die vertikale Betrachtung Rückschlüsse auf die Konsistenz der Einstufungen innerhalb eines Filmkatalogs zulässt.

Als Ergebnis der Auswertung der Kriterienmatrix lässt sich festhalten, dass weder ein Einzelkriterium, noch eine Zweier-Konstellation, noch die meisten Dreier-Konstellationen ausreichend sind, um einen vollen Konsens bezüglich der „Neuseeländischkeit“ eines Films zu erreichen. Dass beispielsweise mit Her Majesty (200I) ein und derselbe Film von der IMDb als „neuseeländisch“, von Conrich als „Koproduktion“ und von der NZFC als ,ausländisch“ eingestuft wird, illustriert einmal mehr den Konstruktcharakter des nationalen neuseeländischen Kinos.

Trotz dieser mitunter arbiträr erscheinenden Zuordnungspraxis zeigt die Kriterienmatrix auch, dass es insgesamt durchaus wichtigere und weniger wichtige Kriterien bei der Beurteilung eines Films als neuseeländisch/nicht-neuseeländisch gibt. Während die Häufigkeiten einiger Kriterienkonstellationen wie diejenige der Filme The Bounty oder Bad Blood derart selten sind, dass das Ergebnis „Dissens“ kaum als repräsentativ angesehen werden kann (aus diesem Grund nur in Klammern gesetzt), ergibt sich für die Konstellation „neuseeländische Produktionsfirmaneuseeländischer Regisseur-neuseeländischer Drehbuchautor-ausländische location“ ein voller Konsens. Allerdings ist auch diese Konstellation mit einer Prävalenz von lediglich 2,5\% selten und ebenfalls kaum als repräsentativ zu erachten.

Erst die Vierer-Konstellation bewirkt einen vollen Konsens und ist mit einer Häufigkeit von fast $80 \%$ die wichtigste Konstellation. Vor allem erzeugt diese Konstellation (relativ) unabhängig vom Filminhalt einen Konsens darüber, dass die betreffenden Filme als „neuseeländisch“ 
einzustufen sind, da beispielsweise Whale Rider zweifelsohne ein neuseeländisches Filmthema hat, wohingegen die Lord of the Rings-Trilogie ebenso unzweifelhaft keinen neuseeländischen Filminhalt hat. Diese Feststellung steht nicht im Widerspruch zu der weiter oben getroffenen Aussage, dass der Filminhalt kaum quantifizierbar sei, da diese Problematik nur für „inhaltliche Grenzfälle“ gilt. Bei den Filmen Whale Rider und Lord of the Rings ist die Bestimmung, ob der Inhalt neuseeländisch oder ausländisch bzw. Fantasy ist, aber sehr eindeutig zu treffen. Dies bedeutet letztendlich, dass die Produktionsbedingungen wichtiger sind als der Filminhalt, wenn es in der Praxis darum geht, zu bestimmen, ob ein Film neuseeländisch ist oder nicht. Hiermit soll nicht suggeriert werden, dass der Filminhalt bei der nationalen Zuordnung unbedeutend sei, da viele Filme der Viererkonstellation einen eindeutig neuseeländischen Inhalt haben. Im Zweifelsfall ist jedoch die Produktionsebene ausschlaggebender als die inhaltliche Ebene.

Wichtig bei der Zuordnung sind aber auch das Prestige eines Films und dessen finanzieller Erfolg, Kriterien, die in Einzelfällen wichtiger als die vorgenannten Aspekte sein können. So werden sehr erfolgreiche Filme wie Lord of the Rings trotz des nicht-neuseeländischen Filminhalts und The Piano trotz ausländischer Produktionsfirmen und einer ausländischen Regisseurin/ Drehbuchautorin gleichermaßen als „neuseeländisch“ eingestuft. Mit „Prestige“ ist vor allem die positive Bewertung durch professionelle Filmkritiker gemeint, unabhängig vom finanziellen Erfolg, der vor allem bei art house-Filmen meist gering ist. „Finanzieller Erfolg“ bezieht sich hingegen in erster Linie auf Filme, die mit dem Ziel produziert wurden, „Blockbuster“ zu werden. Diese beiden Kriterien gelten allerdings vorwiegend in „positiver“ Hinsicht, d. h. sofern ein Film eines der beiden Kriterien erfüllt, können andere Aspekte in den Hintergrund treten, wohingegen ein negativ bewerteter Film oder ein „Kassenflop“ wie beispielsweise Luella Miller nicht aufgrund seines mangelnden Erfolgs aus dem neuseeländischen Filmoeuvre ausgeschlossen wird. Eine Ausnahme bildet die Einstufung von Mesmerized als ,ausländische“ Produktion im NZFC-Filmkatalog, obwohl dieser Film dieselbe Kriterienkonstellation aufweist wie The Piano, der wiederum von der NZFC als „neuseeländisch“ eingestuft worden ist. Da die Filme sogar inhaltliche Parallelen aufweisen, erscheint die Einstufungspraxis der NZFC auf den ersten Blick arbiträr, lässt sich aber mit oben genannten Prestigeinteressen erklären, da Mesmerized trotz der Mitwirkung des Stars Jodie Foster schlechte Kritiken erhielt und auch kein finanzieller Erfolg war. Da aber nur verhältnismäßig wenige Filme des neuseeländischen Filmoeuvres in den neuseeländischen Kinos (siehe Abbildung 2.5.2) und auf globaler Ebene erfolgreich sind, spielen diese Faktoren bei den meisten Filmen keine Rolle. 
Eines der wichtigsten Ergebnisse der Analyse ist die überragende Bedeutung der neuseeländischen location im Diskurs um ein nationales neuseeländisches Kino. Dass die location einen Sonderstatus innehat, wird bereits daraus ersichtlich, dass in den Filmkatalogen von Conrich, Martin und der NZFC eine Extrakategorie für als ausländisch eingestufte Produktionen mit neuseeländischer location vorhanden ist, was z. B. durch Anmerkungen wie „only locations“ erkennbar ist. Solcherlei Extrakategorien werden jedoch nicht für beispielsweise ausländische Produktionen mit neuseeländischem Regisseur durch Anmerkungen wie „only [New Zealand] director“ eingesetzt.

Aus der Kriterienmatrix lässt sich hinsichtlich der location ableiten, dass mit Ausnahme der vierten Dreier-Konstellation in allen Konstellationen eine neuseeländische location involviert ist, was darauf schließen lässt, dass dieser Faktor isoliert betrachtet der wichtigste ist. Diese Feststellung wird durch zwei weitere Ergebnisse bestätigt: Zum einen sind bei der vierten Dreier-Konstellation, die keinen neuseeländischen Drehort aufweist, alle anderen Faktoren neuseeländisch. Es gibt keine Konstellation, bei der bei einem Film mit ausschließlich ausländischer location auch ein weiterer Faktor - beispielsweise der Regisseur - ausländisch wäre und der Film dennoch als neuseeländisch eingestuft worden wäre. Dies bedeutet, dass nur aufgrund der Neuseeländischkeit aller anderen Faktoren trotz fehlender neuseeländischer locations diese Filme als neuseeländisch eingestuft wurden.

Zum anderen stellt die einzige Einer-Konstellation das genaue Gegenteil der eben genannten Dreier-Konstellation dar: Obwohl in der Einer-Konstellation weder die Produktionsfirma, noch der Regisseur, noch der Drehbuchautor neuseeländisch sind, werden die betreffenden Filme als neuseeländisch eingestuft, wenn auch zumeist als Koproduktionen. Da bei Filmen wie Last $\mathrm{Sa}$ murai und Willow nicht einmal der Handlungsort neuseeländisch ist, scheint allein die location hier der Faktor gewesen zu sein, der in den Augen der Filmkatalogersteller die Einstufung als „neuseeländisch" gerechtfertigt hat.

Die zentrale Bedeutung der location lässt sich damit erklären, dass gerade die einzigartige Landschaft ein zentraler Teil der nationalen Identität Neuseelands ist und außerdem bei den verstärkten nation branding-Bemühungen Neuseelands eine überaus wichtige Rolle spielt. Nation branding stellt eine konzertierte, strategisch gebündelte Werbeaktion dar, die darauf abzielt, eine Nation in ein Produkt zu verwandeln, um damit den kommerziellen Gewinn, z. B. durch Tourismus, zu maximieren, was in einer globalisierten, von kapitalistischen Prinzipien geleiteten Welt letztlich unabdingbar geworden ist (Bell 2005: I3). Hierbei wird gezielt auf die touristischen Erwar- 
tungen eingegangen und auf besonders ansprechende nationale Mythen und Stereotypen rekurriert und diese zumeist in stark vereinfachter Form in die nation brand inkorporiert (Huijibens 20II: 575). Huijibens merkt an, dass gerade Inseln eine Aura des Isolierten, Fernen und Mystischen haben (554), was natürlich auch für Neuseeland gilt und als Unique Selling Proposition propagiert werden kann. Tatsächlich wurde Neuseeland bereits früh touristisch vermarktet als fernes „scenic wonderland“ (Bell 2005: I8), was einen der Grundsteine für das spätere clean EG green-Image und die $100 \%$ Pure New Zealand-Kampagne legte. Wie Leotta anführt, wurde das clean Ef green-Image mit Bildern aus dem Film The Piano erweitert, da der Film sehr erfolgreich war und Filmtourismus generiert hatte (20II: 90-93). Das durch nation branding erzeugte Image einer Nation kann hierbei durchaus von der nationalen Bevölkerung internalisiert und in die bestehende nationale Identität inkorporiert werden (Bell 2005: 16). Obwohl nation branding demnach eine vorwiegend nach außen gerichtete Maßnahme ist, kann diese auch nach innen wirken - was nicht zuletzt an den besonders „griffigen“, verdichteten Bildern liegt, die das nation branding im Zuge der Vereinfachung nationaler Mythen und Stereotypen erzeugt.

Auch in Filmen, wo der Handlungsort ausländisch ist, stellt die neuseeländische location eine im Hintergrund wirkende, ästhetische Instanz dar, die eine ungleich stärkere ZuschauerAssoziation mit Neuseeland ermöglicht (und ggf. zu Filmtourismus führt) als beispielweise die Mitwirkung einer neuseeländischen Produktionsfirma. Eine neuseeländische location stellt einen wichtigen Aspekt dar, wenn es darum geht, einem Film einen „significant New Zealand content“ zuzuschreiben. Aufgrund dieser großen Bedeutung der neuseeländischen location wird im dritten Kapitel die Rolle der location und ihr Bezug zum Handlungsort eingehend analysiert werden.

Abschließend lässt sich folgende Hierarchie der Kriterien in der Kategorisierungspraxis erkennen: Bei der Einstufung eines Films als neuseeländisch oder ausländisch ist das Kriterium „location“ das wichtigste, ggf. gefolgt von den Kriterien „Prestige“ und „finanziellem Erfolg“, die im Zweifelsfall einen Teil der restlichen Kriterien „Regisseur“, „Drehbuchautor“, „Produktionsfirma“ und auch „Handlungsort“" verdrängen können.

Während bis jetzt vorwiegend die Aspekte einer „subjektiven“ De- und Renationalisierung untersucht worden sind, soll nun die „objektive“ De- und Renationalisierung analysiert und vergleichend gegenübergestellt werden. ${ }^{23}$ Dazu werden die einzelnen Unterebenen „Regisseur“, „Pro-

\footnotetext{
${ }^{23}$ Mit „objektiv“ soll in diesem Zusammenhang natürlich nicht suggeriert werden, dass beispielsweise die Nationalitäten der Filmemacher „unumstößliche Naturgesetze“ wären. Allerdings ist die normative Festlegung, dass eine Person mit neuseeländischer
} 
duktionsfirma“, „Drehbuchautor“ und „location“ separat erörtert und daraufhin analysiert, wie viel Prozent davon (ggf.) faktisch neuseeländische Minderheit, neuseeländisch(-europäisch), neuseeländisch-ausländisch sowie ausländisch sind. Anhand der Darstellung des zeitlichen Verlaufs sollen eventuelle Trends aufgezeigt und interpretiert werden. Die grafisch dargestellten Werte sind immer in Prozent angegeben, d. h. sie verhalten sich relativ zu der Gesamtanzahl produzierter Filme in einem Jahr, da absolute Werte einen verzerrten Eindruck bezüglich der De- und Renationalisierung einzelner Sektoren geben würden. Analysegrundlage sind die Daten und Informationen der Internet Movie Database. Im Folgenden wird der Sektor „Produktionsfirma“ analysiert: 


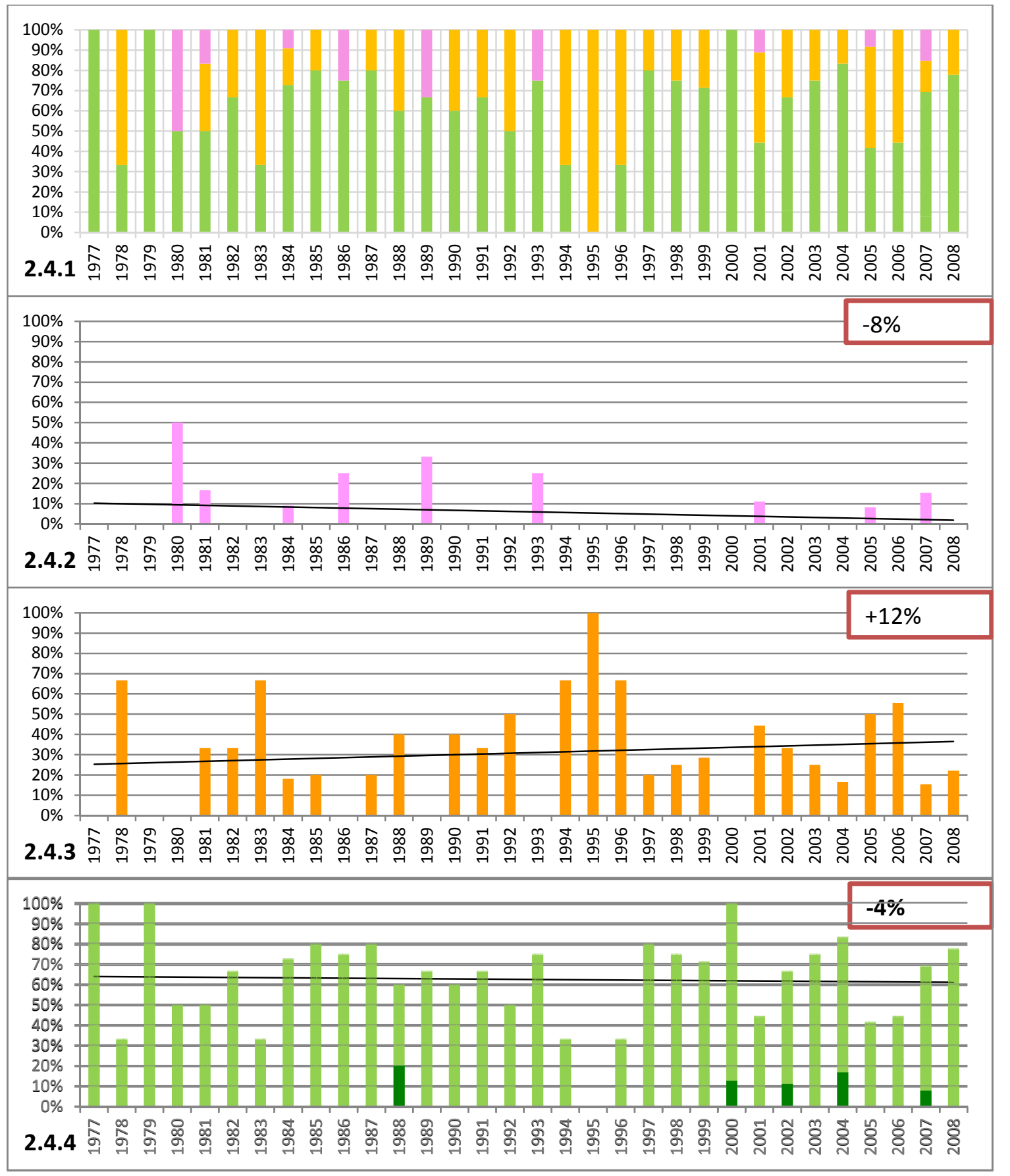

\section{Abbildung 2.4: Entwicklung des Sektors}

"Produktionsfirma“,

\section{Übersicht und getrennt nach Typ}

\section{Legende:}

dunkelgrün $=$ Produktionsfirma einer nationalen Minderheit hellgrün = neuseeländische Produktionsfirma orange $=$ Koproduktion

rosa = ausländische Produktionsfirma

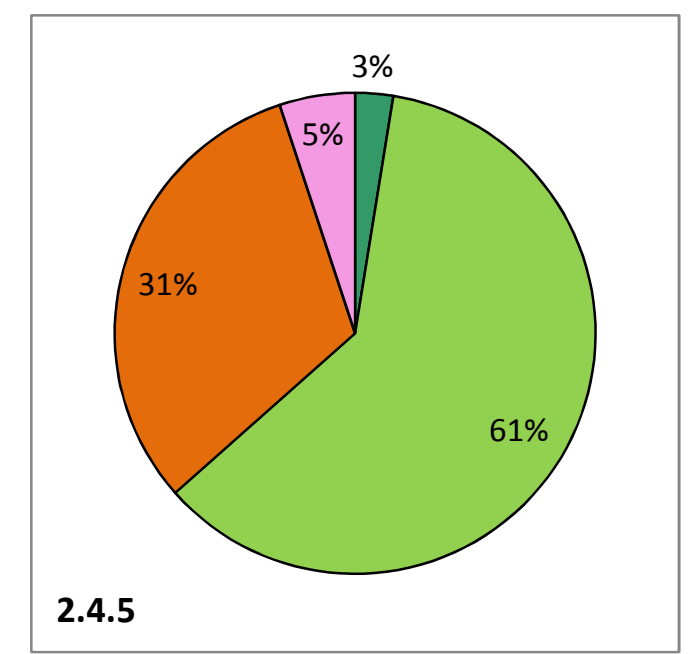


In Abbildung 2.4 ist in Diagramm 2.4.I als Übersicht die relative Anzahl der Filme (Y-Achse) pro Jahr (X-Achse) dargestellt, in denen nur neuseeländische Produktionsfirmen, Koproduktionen oder nur ausländische Produktionsfirmen involviert gewesen sind. So ist beispielsweise zu sehen, dass I0o \% der 1977 produzierten und im Filmoeuvre angeführten Filme ausschließlich von neuseeländischen Produktionsfirmen produziert worden sind, während 1978 der Anteil auf ca. $35 \%$ gesunken ist und von Koproduktionen dominiert wird (ca. 65\%). Bereits 1980 gibt es erste Filme, an denen ausschließlich ausländische Produktionsfirmen mitgewirkt haben, was in etwa mit dem Beginn der zuvor besprochenen subjektiven Denationalisierung korreliert (I98I, s. o.). Insgesamt ist erkennbar, dass Filme mit rein neuseeländischen Produktionsfirmen im gesamten Zeitraum zwar deutlichen Schwankungen unterworfen sind, aber dennoch dominieren. Nur um das Jahr 1995 ist der Anteil an Koproduktionen außerordentlich hoch. Im Trend zeigt sich, dass die Anzahl rein neuseeländischer Produktionen (nur bezogen auf die Produktionsfirmen) mit durchschnittlich $-4 \%$ eher geringfügig abgenommen hat (2.4.4), also weitgehend stabil geblieben ist, während der Anteil rein ausländischer Produktionen mit $-8 \%$ relativ deutlich abgenommen hat (2.4.2), was als faktische Renationalisierung gewertet werden kann. Allerdings wird dieser Trend durch den starken Anstieg an Koproduktionen um I2 \% wieder relativiert (2.4.3), da an Koproduktionen immer auch ausländische Firmen beteiligt sind. Insgesamt hat sich dieser Sektor durch die deutliche Zunahme an Koproduktionen also partiell denationalisiert.

Vor allem zeigt sich, dass es praktisch seit den Anfängen des zeitgenössischen neuseeländischen Kinos Koproduktionen gegeben hat und die Denationalisierung daher kein wirklich neuer Trend ist, was sich auch an der statischen Darstellung in Diagramm 2.4.5 erkennen lässt: Bei rund $36 \%(3 \mathrm{I} \%+5 \%)$ der Filme sind ausländische Produktionsfirmen involviert gewesen. Dass die Denationalisierung in diesem Sektor nicht noch stärker vorangeschritten ist, liegt vor allem an den Renationalisierungsbemühungen der New Zealand Film Commission, die an $37 \%$ der Filmproduktionen beteiligt (Abbildung 2.5.I) und oftmals der Hauptproduzent gewesen ist. Die nahezu durchgängige Denationalisierung lässt sich insbesondere dadurch erklären, dass das zeitgenössische neuseeländische Kino (wie auch die neuseeländische Nation an sich) sehr jung ist und seine Entstehung in eine Zeit fällt, wo sich der Prozess der Globalisierung (selbst in seiner engen Definition) bereits deutlich bemerkbar macht, hier vor allem in der Form einer Vormachtstellung des Hollywoodkinos in Neuseeland (Abbildung 2.5.2): 


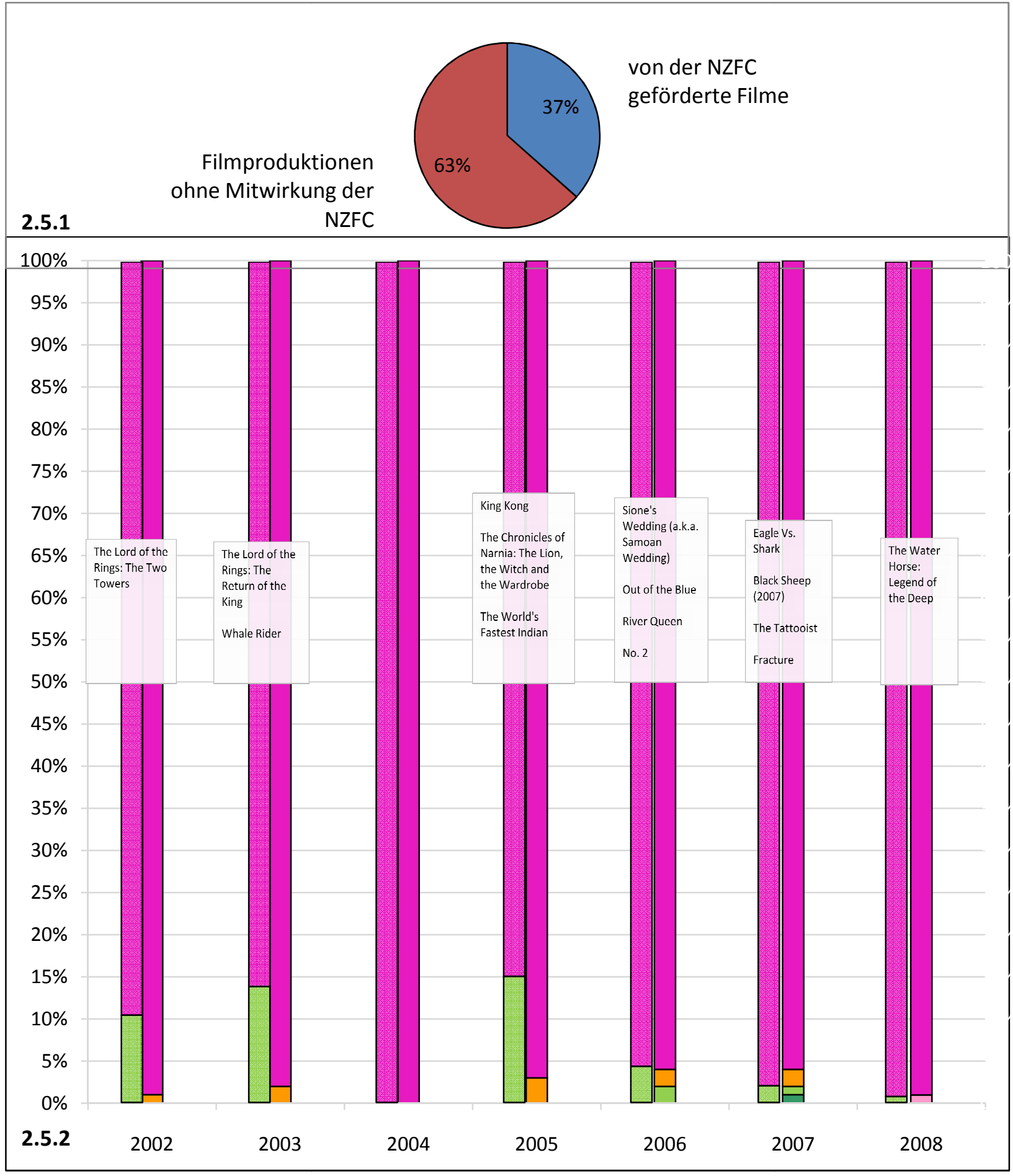

Abbildung 2.5: Förderanteil der New Zealand Film Commission in \% (2.5.I) und Anteil neuseeländischer Filme am neuseeländischen Kinomarkt (Top ıоo) - Umsatzanteile in \% und Filmanzahl in \% (2.5.2)

\section{Legende:}

Jeweils linke Balken:

hellgrün = von neuseeländischen Filmen erwirtschafteter Umsatz auf dem neuseeländischen Kinomarkt rosa $=$ von ausländischen Filmen erwirtschafteter Umsatz auf dem neuseeländischen Kinomarkt

Jeweils rechte Balken:

dunkelgrün = neuseeländischer Film, an der Produktionsfirma einer nationalen Minderheit involviert war hellgrün $=$ neuseeländischer Film unter ausschließlicher Beteiligung neuseeländischer Produktionsfirmen orange $=$ neuseeländische Koproduktion; hellrosa $=$ neuseeländischer Film unter ausschließlicher Beteiligung ausländischer Produktionsfirmen; rosa = ausländischer Film 
In Abbildung 2.5.2 sind für den Zeitraum 2002-2008 der quantitative Anteil neuseeländischer Filme am neuseeländischen Kinomarkt ${ }^{24}$ (Top ıоo) sowie die Umsatzanteile in \% dargestellt. Die Umsatzzahlen für den Zeitraum vor 2002 waren nicht zu ermitteln, da Boxoffice Mojo die Statistik erst seit 2002 führt.

Für jedes Jahr im Zeitraum 2002-2008 sind jeweils zwei gestapelte Säulen dargestellt, wobei die jeweils linke den Umsatzanteil neuseeländischer Filme am Top ıoo-Filme-Gesamtumsatz auf dem neuseeländischen Kinomarkt darstellt. Die rechte Säule zeigt zum Vergleich den prozentualen Anteil neuseeländischer Filme (unter den Ioo finanziell erfolgreichsten Filmen im Jahr). So ist zu entnehmen, dass 2002 die neuseeländisch-amerikanische Koproduktion Lord of the Rings: The Two Towers io \% des Gesamtumsatzes erwirtschaftet hat, wobei dieser Film anzahlmäßig lediglich I \% der Top ıoo Filme ausmacht. 2003 kann der Umsatzanteil sogar auf knapp I4 \% erhöht werden, unter Mitwirkung des Überraschungserfolgs Whale Rider. Während 2004 kein einziger neuseeländischer Film unter den Top Ioo vertreten ist, erzeugen 2005 gleich drei neuseeländische Filme einen Umsatzrekord von knapp is \%. 2006 lässt sich ein deutlicher Umsatzeinbruch verzeichnen, wobei der rein neuseeländisch produzierte Erfolg Sione's Wedding einen großen Anteil am immer noch relevanten Umsatzanteil von knapp $4 \%$ ausmacht. Der immer noch vergleichsweise hohe Anteil neuseeländischer Produktionen von rund 4\% kann einen weiteren Umsatzrückgang auf nurmehr knapp $2 \%$ in 2007 nicht verhindern. Bemerkenswert ist aber, dass mit Eagle vs. Shark ein Film es erstmals in die Top Ioo geschafft hat, der (unter anderem) von einer nationalen Minderheit produziert worden ist (die von Taika Waititi gegründeten Whenua Films). 2008 ist nur noch ein als neuseeländisch eingestufter Film vertreten, der zudem ausschließlich von ausländischen Produktionsfirmen produziert wurde und der den weiteren Umsatzrückgang auf lediglich i \% nicht aufhalten kann.

Insgesamt zeigt sich, dass die anfangs disproportional hohe Umsatzgenerierung neuseeländischer Filme sich umgekehrt hat, seitdem Blockbuster wie die Lord of the Rings-Trilogie, Chronicles of Narnia und King Kong aus dem Kinoprogramm genommen worden sind und daher im finanziellen Sektor seit 2006 von einer Denationalisierung gesprochen werden muss, was auch bedeutet, dass Hollywood den neuseeländischen Kinomarkt wieder stärker dominiert.

Während bis jetzt die „externe“ Entwicklung des nationalen Kinos hinsichtlich ausländischer Produktionseinflüsse analysiert worden ist, soll zum Abschluss der Analyse der Unterebene

\footnotetext{
${ }^{24}$ Der neuseeländische Kinomarkt setzt sich aus sämtlichen in neuseeländischen Kinos ausgestrahlten Filmen zusammen, d. h. sowohl neuseeländischen als auch rein ausländischen Produktionen.
} 
„Produktionsfirma“ die nationsinterne Situation indigener Produzenten, d.h. der Māori sowie weiterer kultureller Minderheiten analysiert werden. Im Rahmen dieser Arbeit wird „nationale Minderheit" als Machtminderheit im postkolonialen Sinne definiert und nicht im rein quantitativen Sinne, d. h. dass beispielsweise Neuseelanddeutsche, -kanadier oder -schweizer usw. hier nicht behandelt werden, da sie als „weiße“ bzw. Pākehā (aus Māori-Sicht) klassifiziert werden können. Stattdessen ist der Fokus auf „Nicht-Weiße“, insbesondere auf Māori, Samoaner, Fidschiianer, Inder und Chinesen gerichtet, da diese Bevölkerungsgruppen meist ebenfalls aus einem Land mit postkolonialer Historie stammen (beispielsweise Indiens Kolonialisierung durch die Briten). Dem Report „New Zealand in Profile“ der Statistics New Zealand zufolge setzt sich die kulturelle Identität Neuseelands im Jahre 2009 aus 67,6 \% Europäern, I4,6 \% Māori, 9,2 \% Asiaten, 6,9\% Pacific Peoples, I2,I \% anderen Ethnien zusammen, was den multikulturellen Charakter Neuseelands widerspiegelt.

Während es mittlerweile eine Vielzahl neuseeländischer Filme gibt, die auf inhaltlicher Ebene die Situation diverser kultureller Minderheiten in Neuseeland darstellen und auch in puncto Umsatzgenerierung besonders Erfolg versprechend sind, illustriert Abbildung 2.4.5, dass Minderheiten im Produktionssektor (zumindest im analysierten Zeitraum) weiterhin unterrepräsentiert sind: Während kulturelle Minderheiten mittlerweile mit rund $30 \%$ fast ein Drittel der neuseeländischen Gesamtbevölkerung ausmachen, sind lediglich 3\% der neuseeländischen Produktionsfirmen in der Hand von Minderheiten. Konkret sind dies: Awatea Films, He Taonga Films, Whenua Films und Rice Digital. Abgesehen von Rice Digital, einer vom indischstämmigen Amabir Singh gegründeten Produktionsfirma, sind die restlichen Firmen Māori-verwaltet. Während der Anteil der Māori an der Gesamtbevölkerung seit rund 2o Jahren nahezu konstant bleibt (bei durchschnittlich I5 \%, Abbildung 2.6), hat die Involvierung von Māori im Produktionssektor zugenommen, wenn auch auf sehr niedrigem Niveau. Abbildung 2.4.4 zeigt in der Entwicklung einen leicht positiven Trend: ${ }^{25}$ Während vor 2000 lediglich der Film Mauri von einer nationalen Minderheit produziert worden ist (mit finanzieller Unterstützung der NZFC), ist die Involvierung von MinderheitenProduktionsfirmen ab 2000 deutlich häufiger geworden, wobei die vom Māori Taika Watiti gegründete Firma Whenua Films mit den überdurchschnittlich erfolgreichen Filmen Whale Rider und Boy (2009) besonders hervorzuheben ist. Dieser Trend ist als Renationalisierung zu werten, da Māori als integraler Bestandteil in der neuseeländischen Kultur verankert sind.

\footnotetext{
${ }^{25}$ Die Angabe von „-4\%“ in Abbildung 2.4.4 bezieht sich hingegen auf die Entwicklung der neuseeländischen Produktionsfirmen an sich, also den neuseeländisch-europäischen und den von Minderheiten geleiteten Produktionsfirmen zusammengenommen.
} 


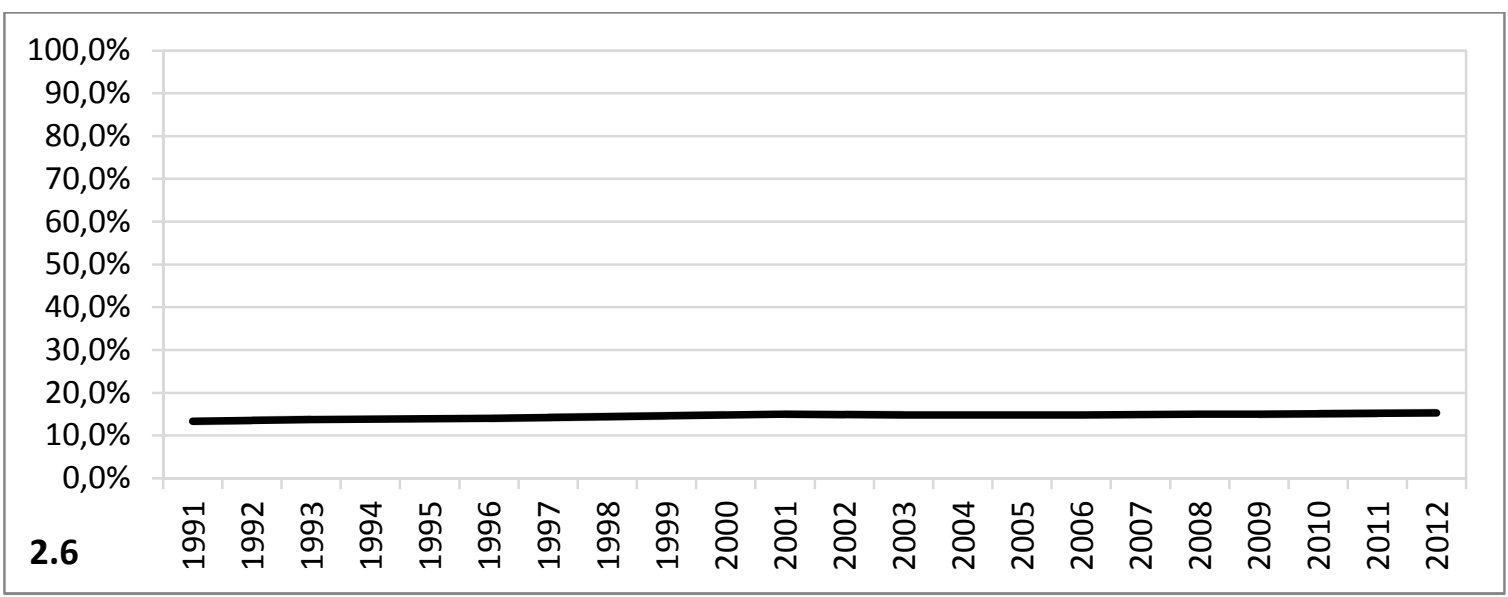

Abbildung 2.6: Entwicklung Bevölkerungsanteil Māori, I99I-20I2

Im Vergleich zur Entwicklung der Unterebene „Produktionsfirma“ illustriert Abbildung 2.7, dass sich die Unterebene „Regisseur“ nur geringfügig verändert hat und daher gegenüber Globalisierungseinflüssen resistenter zu sein scheint: 

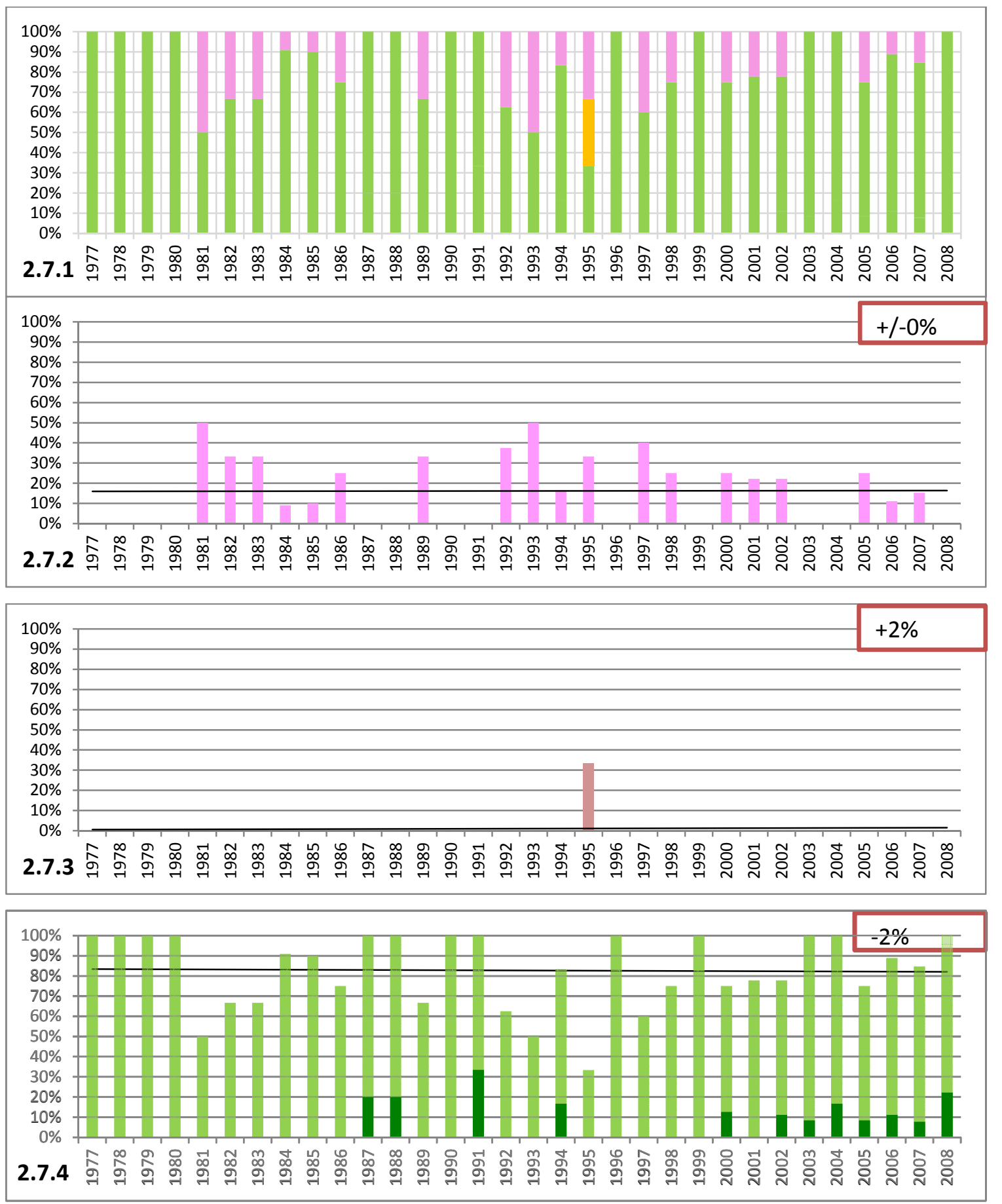

Abbildung 2.7: Entwicklung des Sektors

„Regisseur“,

Übersicht und getrennt nach Typ

Legende:

dunkelgrün $=$ Regisseur, der einer nationalen Minderheit angehört

hellgrün = neuseeländische Nationalität

orange $=$ Zusammenarbeit eines neuseeländischen

Regisseurs mit einem ausländischem Regisseur

rosa $=$ ausländischer Regisseur

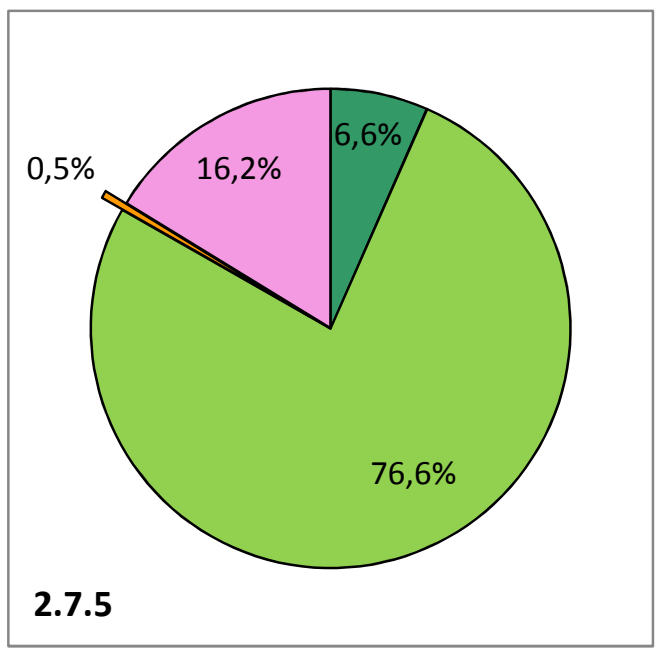


Während die Zusammenarbeit mehrerer Produktionsfirmen oder Drehbuchautoren durchaus üblich ist, ist die Zusammenarbeit mehrerer Regisseure wie bei der Produktion des Films The Offering (1995) (Kollaboration zwischen Francesca Fisher [Mexiko] und Taggart Siegel [Neuseeland]) eine atypische Situation und daher sehr selten. Dies liegt vor allem daran, dass der einzelne Regisseur traditionell eine zentrale Rolle in der künstlerischen Umsetzung eines Films spielt, was sich auch in der früher verbreiteten Akzeptanz der Auteur-Theorie in der filmwissenschaftlichen Forschung widerspiegelt.

Die leichte Abnahme von Filmen mit neuseeländischen Regisseuren liegt vor allem darin begründet, dass aufgrund der besseren Karrieremöglichkeiten einige Regisseure nach Hollywood abgewandert sind und später mit amerikanischer Nationalität temporär nach Neuseeland zurückkehren und dort in neuseeländischen Filmproduktionen mitwirken, diesmal allerdings als ausländische Regisseure (Conrich/Murray 2008: 6-8). So hat beispielsweise Roger Donaldson den Film Sleeping Dogs (1977) als neuseeländischer Regisseur gedreht, während er bei dem Dreh von The World's Fastest Indian (2005) mit Anthony Hopkins bereits die amerikanische Staatsbürgerschaft innehatte. Der beklagte „cinematic brain-drain“ in der neuseeländischen Filmindustrie lässt in den Augen vieler Zuschauer Rückschlüsse auf den Grad der „nationalen Verbundenheit" (ehemals) neuseeländischer Regisseure zu und hat insbesondere unter neuseeländischen Zuschauern mitunter zur Kritik an der eigenen Filmindustrie sowie an den betreffenden Regisseuren geführt. So sind neben Donaldson auch Geoff Murphy (u.a. Goodbye, Pork Pie), Vincent Ward (u.a. Vigil) und Lee Tamahori (u. a. Once Were Warriors) dem Ruf Hollywoods gefolgt.

Vor diesem Hintergrund ist die Zelebrierung Peter Jacksons als Nationalheld kaum verwunderlich, da er Hollywood quasi nach Neuseeland gelockt hat und in den Augen vieler Zuschauer seinem Heimatland „treu“ geblieben ist, obwohl gerade ein Film wie Lord of the Rings auf der Produktionsebene und aufgrund seines national unspezifischen Fantasysettings besonders transnational ausgerichtet ist. Allerdings ist mit dem Produktionsdebakel um den Hobbit auch Peter Jackson in die Kritik geraten und seine früher gelobte „nationale Treue“ infrage gestellt worden. $^{26}$

Im Vergleich zum Sektor „Produktionsfirma“ zeigt sich auch hier, dass ausländische Regisseure über den gesamten Zeitraum ziemlich regelmäßig an neuseeländischen Filmen mitgewirkt

\footnotetext{
${ }^{26}$ Die „Hobbit-Krise“ wird im zweiten Kapitel genauer erörtert werden.
} 
haben und dass dieser Sektor daher ebenfalls von leichter Denationalisierung geprägt worden ist, wenn auch die zeitlichen Veränderungen weniger deutlich sind.

Hinsichtlich der Mitwirkung nationaler Minderheiten ist der Anteil an der Gesamtproduktion mit 6,6 \% (Abbildung 2.7.5) deutlich höher als bei den Produktionsfirmen. Hier sind vor allem Barry Barclay und Merata Mita hervorzuheben, die als Pioniere des indigenen neuseeländischen Kinos, dass von Barclay selbst als Fourth Cinema ${ }^{27}$ bezeichnet wird, betrachtet werden können: Barclays 1987 ausgestrahlter Film Ngati ist einer der ersten Filme überhaupt mit einem Māori als Regisseur. Bereits ein Jahr später folgt mit Mauri der erste Film, der unter alleiniger Regie einer Māori-Frau, Merata Mita, gedreht worden ist (To Love a Māori (1972) entstand unter der Regie von Māori-Frau Ramai Hayward, allerdings in Zusammenarbeit mit ihrem Pākehā-Ehemann Rudall Hayward). Nach dem großen Erfolg von Once Were Warriors (1994), unter der Regie von Māori Lee Tamahori gibt es eine Lücke in den Jahren 1995-1999, in denen kein einziger Film von einer nationalen Minderheit gedreht wurde. Ähnlich wie bei den Produktionsfirmen zeigt sich aber auch hier ein eindeutig positiver Trend erst ab dem Jahr 2000, wobei ab 2002 jedes Jahr mindestens ein Film mit einem Regisseur produziert worden ist, der einer nationalen Minderheit zugeordnet werden kann. Während vor der Jahrtausendwende sämtliche Regisseure, die sich nationalen Minderheiten zurechnen lassen, Māori waren, hat sich die Entwicklung ab 2003 deutlich diversifiziert: Nun spielen indische Regisseure wie die Geschwister Jitendra und Promla Pal (Gupta vs. Gordon), Amabir Singh (Inite) und Amit Tripuranemi (Memories of Tomorrow), fidschistämmige Regisseure wie Toa Fraser (No. 2, Dean Spanley) und seit 2008 auch Samoaner wie Sima Urale (Apron Strings) eine immer bedeutendere Rolle in der neuseeländischen Filmindustrie. Dennoch ist in Gesamtsicht fast jeder zweite Minderheiten-Regisseur ein(e) Māori, was nicht zuletzt aus dem Umstand zu erklären ist, dass Māori-Regisseure seit Beginn des zeitgenössischen neuseeländischen Kinos von der NZFC besonders gefördert wurden.

Im Sektor „Drehbuchautor“ hat es wiederum ähnlich wie im Sektor „Produktionsfirma“ deutliche Veränderungen gegeben, was die Involvierung ausländischer Drehbuchautoren anbetrifft:

\footnotetext{
${ }^{27}$ Barry Barclay. „Exploring Fourth Cinema.“ Summer School lectures. Reimagining Indigenous Cultures: The Pacific Islands. Summer Institute: Hawaii, 23. Juli 2003: I6. zitiert in: Leotta, 20II: I08.
} 


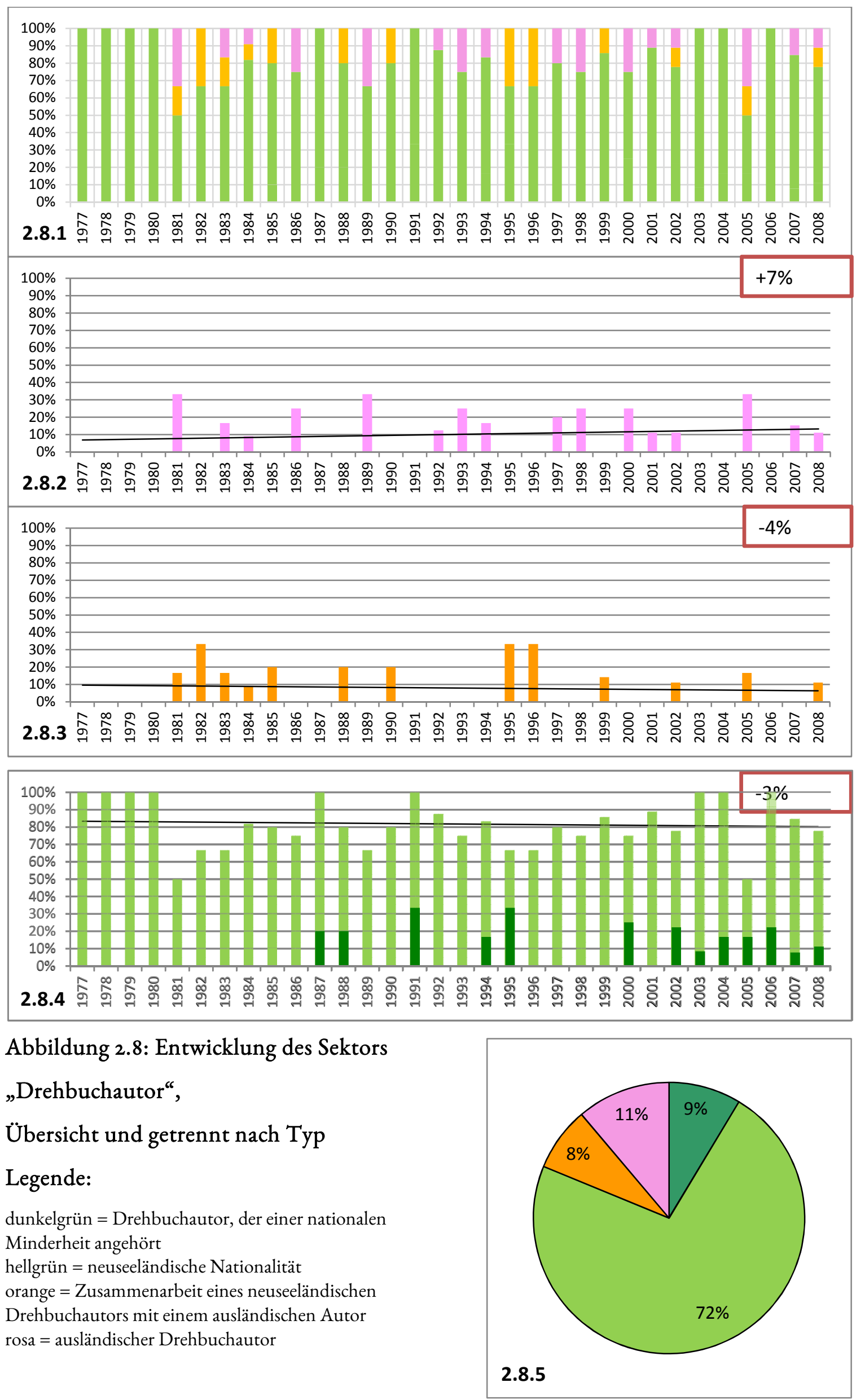


Die Anzahl von Filmen, an denen ausschließlich ausländische Drehbuchautoren mitgewirkt haben, ist im Durchschnitt um $+7 \%$ gestiegen, während Filme, an denen sowohl ausländische als auch neuseeländische Drehbuchautoren mitgewirkt haben, um $4 \%$ abgenommen haben. Im Gegensatz zur Arbeit des Regisseurs ist die Zusammenarbeit mit anderen Drehbuchautoren durchaus üblich, sodass der Anteil an „Drehbuch-Koproduktionen“ im Vergleich deutlich höher ist. Insbesondere, wenn die Produzenten der Ansicht sind, dass das Drehbuch eines unerfahrenen neuseeländischen Autors von „minderer Qualität“ sei, werden oft externe Personen aus dem Ausland hinzugezogen und beauftragt, das Skript zu revidieren, insbesondere, um das Drehbuch an die Erwartungen eines globalen Publikums anzupassen.

An den Diagrammen 2.8.2 und 2.8.3 zeigt sich, dass sich die Zahl ausländischer Drehbuchautoren zulasten neuseeländisch-ausländischer Zusammenarbeit erhöht hat. Die Zunahme ausländischer Drehbuchautoren begründet sich vor allem daraus, dass das Drehbuch die Grundlage für einen Film bildet und dessen Qualität daher entscheidend für den Erfolg eines Films ist. In der Tendenz zeigt sich, dass hierzu immer häufiger das Know-how amerikanischer Skriptwriter angefordert wird, die nicht mehr mit neuseeländischen Autoren zusammenarbeiten, sondern diese gleich ersetzen. Peter Jackson argumentierte in einem Interview auf screendaily.com, dass es in einem Land von der Größe Neuseelands kaum mehr als „25 or 30 truly talented screen writers“ gebe und dass diese nur unzureichend von der NZFC gefördert würden (George, 20Io).

Dass sich auch Minderheiten als Drehbuchautoren durchsetzen können, illustriert ihre mit $9 \%$ relativ hohe Beteiligung an der Gesamtproduktion (Abbildung 2.8.5). Hierbei ist die Konstellation Minderheiten-Drehbuchautor mit Minderheiten-Regisseur sehr häufig anzutreffen. So hat die Māori Tama Poata das Drehbuch zu Barry Barclays Ngati geschrieben, während Merata Mita sowohl das Drehbuch zu Mauri als auch die Regie übernommen hat. Ähnlich wie im Sektor „Regisseur“ zeigt sich auch hier ab 2003 eine deutliche Diversifizierung, da nun auch indische, fidschianische und samoanische Drehbuchautoren an Produktionen wie Gupta vs. Gordon (Pal-Geschwister), River Queen (Toa Fraser) und Apron Strings (Shuchi Kothari) mitwirken. Auch dieser Minderheiten-Sektor ist in der Gesamtsicht von Māori-Autoren (rund $50 \%$ ) dominiert.

Während insbesondere Māori-Thematiken bereits im frühen neuseeländischen Film vor I977 sehr populär gewesen sind, was die Bedeutung der Māori-Kultur als ökonomische wie auch kulturelle Ressource für die neuseeländische Filmindustrie unterstreicht, zeichnet sich immer mehr $\mathrm{ab}$, dass die indigene Bevölkerung in den letzten Jahren häufiger selbst als Produzent dieser Filmin- 
halte in Erscheinung tritt. Dies signalisiert eine Abwendung von der paternalistischen Tendenz, als „außenstehender“ Pākehā über indigene Thematiken zu schreiben und eine zunehmende Anerkennung der Autorität indigener Filmemacher, ihre Geschichten selbst filmisch umzusetzen.

Wie bereits im Rahmen der Analyse der subjektiven Denationalisierung angesprochen, ist der abschließend zu analysierende Sektor „location“ ein besonders wichtiger Aspekt des neuseeländischen Films, was auch aus der folgenden Abbildung ersichtlich wird: 

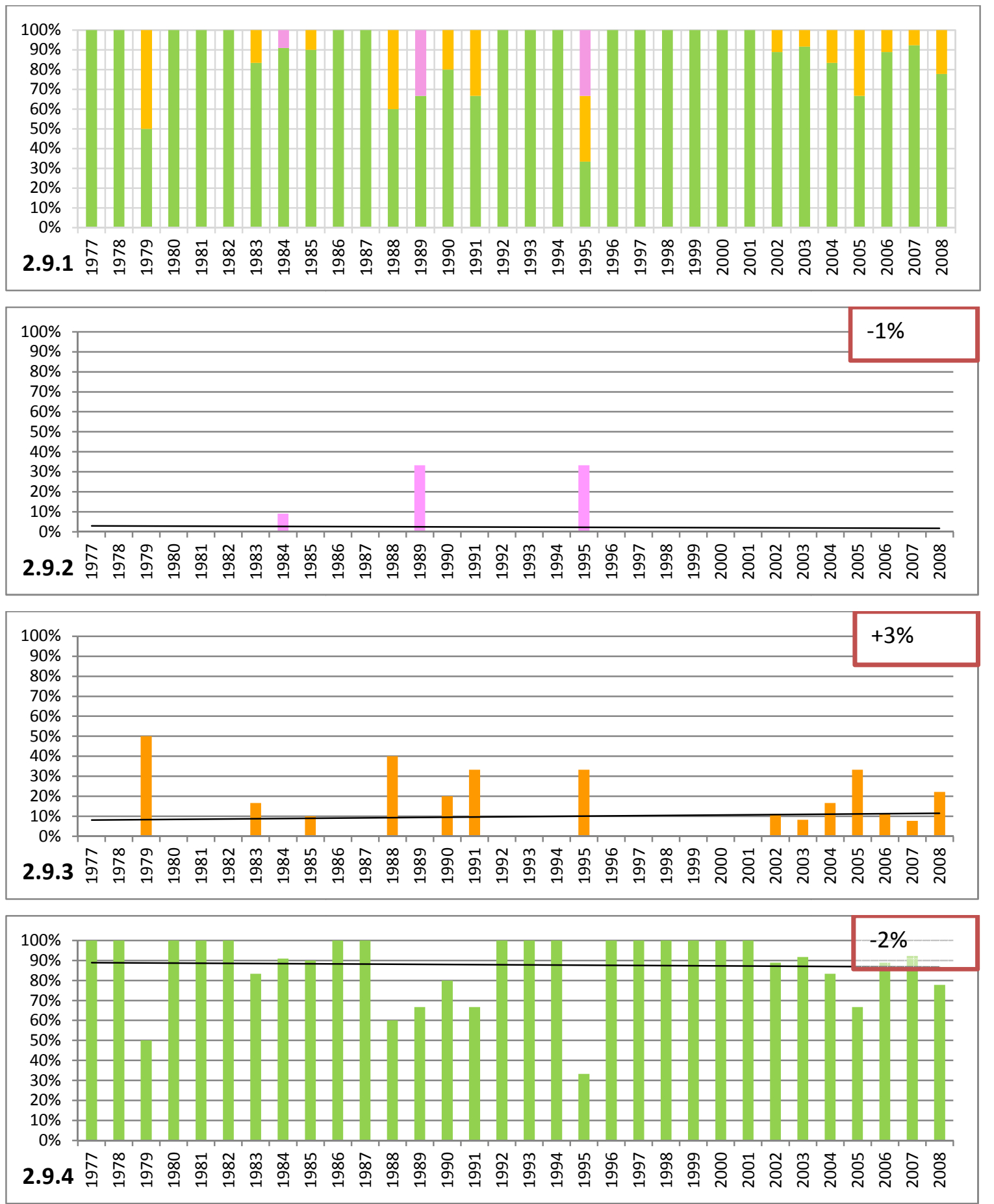

Abbildung 2.9: Entwicklung des Sektors

"location",

\section{Übersicht und getrennt nach Typ}

Legende:

hellgrün = neuseeländische location

orange $=$ ausländische und neuseeländische location

rosa $=$ ausländische location

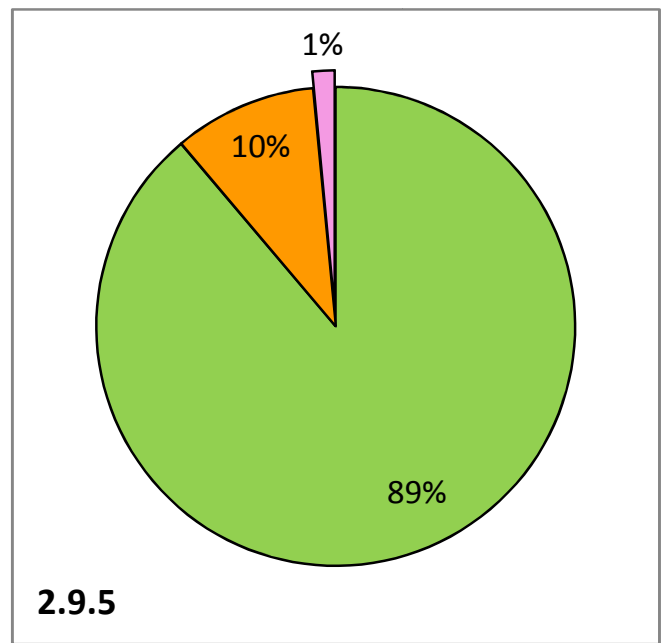


So zeigen die Trendkurve im Diagramm 2.9.4 und das Diagramm 2.9.5, dass der Anteil von Filmen, die eine rein neuseeländische location haben, mit durchschnittlich fast $90 \%$ ausgesprochen hoch ist, während Filme mit neuseeländischen Regisseuren oder Drehbuchautoren mit durchschnittlich rund $83 \%(76,6 \%+6,6 \%)$ bzw. $8 \mathrm{r} \%(72 \%+9 \%)$ (Abbildungen 2.7.5 und 2.8.5) sowie mit rein neuseeländischen Produktionsfirmen (im Mittel nur rund 64\% [6r\% + 3\%]) (Abbildung 2.4.5) deutlich weniger sind. Die Denationalisierung neuseeländischer Filme durch rein ausländische locations ist mit I \% sehr gering und in der Tendenz sehr leicht abnehmend. Dies lässt sich damit erklären, dass - wie die Kriterienmatrix gezeigt hat - der Einsatz neuseeländischer locations einen zentralen Faktor darstellt, wenn es um die Einstufung eines Filmes als „neuseeländisch“ geht. Dennoch ist auch hier ein transnationaler Trend zu erkennen, da es seit 2002 jedes Jahr eine Reihe von Filmen gibt, in denen sowohl auf Neuseeland als auch im Ausland gedreht worden ist (2.9.5). Gerade das Anlocken von runaways nach Neuseeland hat dazu geführt, dass solche Filme zwar in Neuseeland on location gedreht werden, aber immer häufiger ausländische Regisseure, Drehbuchautoren und Produktionsfirmen involviert sind und es dadurch zu Denationalisierung kommt.

\section{4 Überlegungen zur De-/Renationalisierung filmischer Inhalte}

Das vorliegende Kapitel hat sich bis jetzt mit der Frage der De- und Renationalisierung der Produktionsebene neuseeländischer Filme auseinandergesetzt. Was nicht erörtert wurde, ist die Frage nach einer möglichen De- oder Renationalisierung der Inhalte neuseeländischer Filme. Der Inhalt ist zweifellos ein wichtiger Faktor bei der Einstufung eines Films als neuseeländisch / nichtneuseeländisch und wird auch bei der Erstellung der genannten Filmlisten bzw. -kataloge durch die jeweiligen Verfasser eine Rolle gespielt haben. Meines Ermessens sind generalisierbare Aussagen zur De- oder Renationalisierung eines nationalen Kinos in seiner Gesamtheit (statt nur einzelne Filme als "repräsentativ" für das betreffende nationale Kino darzustellen und allein daraus Trends abzuleiten) nur dadurch zu bewerkstelligen, dass sämtliche Filme, die diesem nationalen Kino zugeordnet werden können, auch im Rahmen der Analyse erfasst werden. Dies stellte einen wichtigen Grund für meine Entscheidung dar, mich auf die Produktionsebene zu konzentrieren: Sämtliche, dem neuseeländischen Kino zuordenbare Filme konnten hinsichtlich ihrer Zusammensetzung auf der Produktionsebene relativ unproblematisch empirisch erfasst werden.

Eine ähnlich gelagerte Analyse der Inhaltsebene ist deutlich schwieriger zu bewerkstelligen und meines Wissens bisher auch noch nicht geleistet worden. Martin/Edwards (1997) liefern zwar 
(kurze) inhaltliche Analysen sämtlicher neuseeländischer Filme für den Zeitraum I9I2-1996, es handelt sich hierbei aber in erster Linie um eine Materialsammlung, deren primäres Ziel es nicht ist, übergeordnete Thematiken oder Trends zu ermitteln. Conrich/Murray liefern in ihrem Sammelwerk Contemporary New Zealand Cinema (2008) tiefer gehende Analysen einzelner Aspekte der inhaltlichen Ebene, auch hinsichtlich ihrer Neuseeländischkeit, die jedoch - wohl auch aufgrund der Komplexität und des Umfangs der Analysen - nur eine Auswahl an neuseeländischen Filmen betreffen, viele Filme aber zwangsläufig unbeachtet lassen. Dies dürfte auch einen der Gründe für die Erstellung einer sämtliche Filme umfassenden Filmliste dargestellt haben, die als Anhang geliefert wird, nämlich den Wunsch, dem Leser trotz der Selektivität der Analysen schlussendlich auch einen “Gesamtüberblick” über das neuseeländische Kino bieten zu können und damit auch Filme zu erfassen, die in den Analysen der Verfasser gar nicht behandelt wurden.

Die Frage nach der De- und Renationalisierung der Inhaltsebene des neuseeländischen Kinos kann hier nicht abschließend beantwortet werden. Dennoch sollen zwei grundsätzliche Fragen erörtert werden: Inwiefern könnte man von spezifisch neuseeländischen Filminhalten sprechen? Gibt es Prozesse, die zu einer tendenziellen De- oder Renationalisierung neuseeländischer Filminhalte geführt haben?

Während bei der Analyse der Produktionsebene beispielsweise die Neuseeländischkeit der location zweifelsfrei und quasi-objektiv bestimmbar war, gestaltet sich die Frage nach der Neuseeländischkeit von Filminhalten deutlich schwieriger. Was einen Filminhalt letztlich "neuseeländisch” macht, ist auch von der eigenen Subjektivität abhängig, die notwendigerweise in die Interpretation mit einfließt. Über den Inhalt eines Films, der beispielsweise das historische Ereignis der New Zealand $W$ ars thematisiert, ist ein sehr großer Konsens über die Neuseeländischkeit dieses Filminhalts zu erwarten. Ob aber ein bestimmtes Liebesdrama eine "spezifisch neuseeländische Prägung” hat oder die Filmemacher womöglich sogar bewusst auf einen allzu nationalspezifischen Inhalt verzichtet haben, um ein transnationales Publikum anzusprechen, darüber dürfte es einen deutlich größeren Interpretationsspielraum geben.

Letztendlich aber liefert das neuseeländische Kino eine große Bandbreite inhaltlicher Thematiken, von denen viele relativ unproblematisch als spezifisch neuseeländisch gewertet werden können. Ein inhaltlicher Themenkomplex, der sicherlich als neuseeländisch bezeichnet werden kann, ist die Auseinandersetzung mit der Geschichte der Kolonisation Neuseelands: Filme dieser Kategorie behandeln Themen, die von der frühen Besiedelung Neuseelands (The Piano) über be- 
waffnete Konflikte zwischen Kolonialisten und Māoribevölkerung (Pictures, Utu, River Queen, Tracker) bis hin zur Goldfieberzeit in Neuseeland (Illustrious Energy) reichen. Ebenfalls unproblematisch als inhaltlich „neuseeländisch“ bezeichenbar sind Filme, deren Hauptfokus auf der zeitgenössischen Situation der Māori (Once Were Warriors, What Becomes of the Broken-Hearted?, Whale Rider) und anderer nationaler Minderheiten in Neuseeland (Samoaner: Flying Fox in a Freedom Tree, Siones Wedding, Siones Wedding 2: Unfinished Business; Chinesen: My Wedding and Other Secrets) liegt. Zu dieser Gruppe zählen auch die sogenannten Māori-Filme, d. h. Filme, die nicht nur inhaltlich die Situation der Māori thematisieren, sondern darüber hinaus auch von (und für) Māori produziert wurden und daher eine antihegemoniale Sicht auf die eigene MāoriKultur bieten. Hierzu zählen in erster Linie die Filme von Barry Barclay (Ngati, Te Rua) und Merata Mita (Mauri), aber auch Taika Waititis Boy. Was diese Filme inhaltlich neuseelandspezifisch macht, ist ihre Auseinandersetzung mit der einzigartigen indigenen Kultur der Māori, die sowohl hinsichtlich Neuseelands bikultureller Identität als auch seiner touristischen Vermarktung ein ausgesprochen großes kulturelles und wirtschaftliches Kapital darstellt und ein hohes Differenzierungspotenzial gegenüber anderen Nationen in der globalen Sphäre bietet.

Auch die Thematisierung nationaler Traumata, die aus den Taten einzelner Individuen resultierten, kann als neuseelandspezifischer Inhalt gewertet werden. Gerade in einem Land, dass nicht nur sein auf Umweltschutz bezogenes clean Eீ green-Image vermarktet, sondern sich auch als friedlich und von niedriger Kriminalität geprägt wahrnimmt, haben Amokläufe und grausame Mordfälle besonderes Aufsehen erregt. Die Filme Bad Blood, Heavenly Creatures und Out Of The Blue behandeln derartige Kriminalfälle und weisen explizit darauf hin, dass es sich um reale Geschehnisse in Neuseeland handelt. Jeder dieser Filme präsentiert bewusst zuerst das Image Neuseelands als pastorales Paradies, um es dann anschließend durch die Darstellung irrationaler Gewalttaten zu dekonstruieren. So basiert beispielsweise Out of the Blue auf einem wahren Fall, dem Amoklauf des David Gray, einem arbeitslosen, mental instabilen Waffensammler, der am I4. November 1990 in der auf der Südinsel gelegenen Kleinstadt Aramoana dreizehn Menschen erschoss, bevor er selbst von einer Spezialeinheit erschossen wurde. Während der Anfang des Films die Kleinstadt und die umgebende Küste in betont ruhigen Bildern als verschlafenen, idyllischen Ort zeigt, wechselt die Kameraperspektive während des Amoklaufs in leicht verwackelte Nahaufnahmen, die dem Zuschauer eine klaustrophobische, paranoide Atmosphäre vermitteln sollen. Da der Film auch international rezipiert wurde, wird hier einem globalen Publikum ein Neuseeland ge- 
zeigt, dass den nation branding-Strategien diametral entgegensteht. Trotz der Dekonstruktion eines nationalen Selbstbildes kann diese Art von Dekonstruktion aber nicht wirklich als „Denationalisierung" bezeichnet werden, da der Fokus weiterhin auf nationalen Thematiken verbleibt und zudem das nationale Selbstbild nicht „abgeschafft“, sondern vielmehr „korrigiert“ und durch ein selbstkritischeres ersetzt werden soll.

Eine weitere Gruppe an Filmen, die sowohl in ästhetischer als auch inhaltlicher Hinsicht einen dunklen Gegenentwurf zu Neuseelands vorherrschendem Selbstbild zeichnen, sich dabei aber nicht auf Einzelfälle beschränken, sondern die neuseeländische Gesellschaft an sich als dysfunktional darstellen, sind die Kiwi Gotbic-Filme, wie auch Sam Neill in der Dokumentation Cinema of Unease (1995) anführt. Charakteristische Themen des Kiwi Gotbic sind Conrich zufolge dysfunktionale Familien, psychische Defekte sowie übernatürliche oder monströse Erscheinungen. Conrich zeigt sich erstaunt darüber, dass sich ein Land, das für sein clean EG green-Image bekannt sei, in seiner lokalen Kultur durch eine deutliche Präsenz des Gotbic hervorhebe (Conrich „New Zealand Gothic“, 20I2: 393) und erklärt das Phänomen des Kiwi Gotbic als Reaktion gegen einen „New Zealand way of life, which, for many has appeared so normal, ordered and consummate that it became mundane and predictable““ (40I). Filme wie Bridge to Nowhere, Trial Run, Lost Tribe und Vigil zeigen isolierte Individuen in besonders abgeschiedenen Regionen, die Neuseeland nicht als Paradies, sondern vielmehr als Einöde empfinden und an ihrer Einsamkeit zu zerbrechen drohen. Hierbei wird der Mythos des Man Alone als gewissermaßen spezifisch neuseeländische Variante des amerikanischen Lonesome Cowboy aufgegriffen, wobei ein Film wie Quiet Earth diesen Mythos besonders extrem darstellt und dabei auch die in Neuseeland lange Zeit vorherrschende Angst vor atomaren Konflikten mit den USA thematisiert.

Die Kiwi Gothic-Filme können somit als Ausdruck einer cultural intimacy, einem von Michael Herzfeld geprägten Begriff, interpretiert werden. Mit cultural intimacy bezeichnet Herzfeld Gegendiskurse zu einem offiziellen, von der politischen und kulturellen Elite hochgehaltenen nationalen Selbstbild. Als Beispiel nennt er das „plate smashing“ bei griechischen Hochzeiten, das Ausdruck der persönlichen Freiheit und Unabhängigkeit ist, von der griechischen Regierung aber wiederholt verboten wurde, weil diese Tradition Griechenland gegenüber Ausländern „erniedrige“ und somit in einem schlechten Licht erscheinen lasse (2005: ix-x). Zwar werden Kiwi Gothic-Filme nicht etwa verboten oder zensiert und werden definitiv als Teil des neuseeländischen Kinos anerkannt, dennoch zeichnen diese Filme ein Bild von Neuseeland, das den Bemühungen Neuseelands 
diametral entgegensteht, sich im Rahmen eines nation brandings als paradiesisches, friedliches Land voller glücklicher Menschen (vor allem gegenüber Touristen) zu vermarkten. So spiegelt beispielsweise der Horrorfilm The Lost Tribe die tief liegende Angstfantasie der Pākehā vor der Rache der Māori für vergangenen Landesdiebstahl wider. Der (weiße) Protagonist Edward Scarry bezahlt symbolisch mit seinem Leben für die unzähligen Missachtungen des Tapu-Gebotes. Somit suggeriert der Film ein weiterhin bestehendes, „intimes“ postkoloniales Schuldbewusstsein und zeichnet ein spannungsgeladenes Verhältnis zwischen Pākehā und Māori, das dem offiziellen Bild Neuseelands als einem Land der „racial harmony“ entgegensteht.

Die in Kiwi Gothic-Filmen ebenfalls häufig thematisierte unterschwellige Angst vor einer feindseligen Wildnis steht nicht nur der Zelebrierung der neuseeländischen Wildnis als Ort der Freiheit in Werbefilmen der neuseeländischen Tourismusindustrie entgegen, sondern hat wiederum einen Gegendiskurs provoziert: Die Māori-Filmemacherin Merata Mita bezeichnet die in Kiwi Gothic-Filmen dargestellte Angst der Pākehā vor der neuseeländischen Wildnis als „white neurosis“. Demgegenüber kontrastiert sie Māori-Filme als „driven by identity, resolution and survival“ und einer damit verbundenen deutlich positiveren Landschaftsdarstellung (zit. in Read 2004: 2092Io). ${ }^{28}$ Dies ist in insofern wichtig, als dass die Māori früher aufgrund ihrer religiösen Anschauungen und religiös begründeten Zutrittsverbote (tapu) zu bestimmten Landstrichen von den Kolonialisten als „abergläubisch“ angesehen wurden, die Vorliebe vieler Pākehā-Filmemacher für die Kiwi Gotbic-Ästhetik aber selbst Ausdruck irrationaler Ängste ist. Vor allem aber stellt dies einen Gegendiskurs zu der Kiwi Gotbic-Ästhetik dar, die ja selbst bereits einen Gegendiskurs zum offiziellen nationalen Selbstbild darstellt. Auch die in Māori-Filmen dargestellte Selbstsicht stellt somit eine Form der cultural intimacy dar, da sie nicht mit dem von der Tourismuswerbung vermittelten Bild der Māori übereinstimmt. Dies wird zum Beispiel im Film Ngati dadurch zum Ausdruck gebracht, dass dessen Filmmusik nicht etwa die touristischen Erwartungen an „traditionelle“ Māori-Musik erfüllt, sondern stattdessen moderne E-Gitarrenmusik zum Einsatz kommt und sich auch musikalischer Entlehnungen aus Westernfilmen bedient wird. Durch solche Strategien können MāoriFilme dem Bild einer „zeitlosen“ und starren Māori-Kultur entgegenwirken und diesen Stereotypen das Bild einer sich dynamisch verändernden Kultur entgegensetzen.

Diese Thematiken können mitunter vorherrschende Versionen nationaler Identität infrage stellen und alternative Formen nationaler Identität entgegenstellen - was den grundsätzlichen

\footnotetext{
${ }^{28}$ Merata Mita. „The Soul and the Image.“ Film in Aotearoa New Zealand. Hrsg. Jonathan Denis und Jan Bieringa. Wellington: Victoria UP, 1992. 36-5I (40). Print.
} 
Aushandlungsprozess nationaler Identität illustriert. Inwiefern kann aber nun von einer De- bzw. Renationalisierung der Inhaltsebene des neuseeländischen Kinos gesprochen werden? Conrich und Murray identifizieren verschiedene Wellen des neuseeländischen Kinos: Die new wave startet mit der Gründung der New Zealand Film Commission 1977 und dem Erscheinen von Sleeping Dogs im selben Jahr sowie später folgenden Filmen wie Goodbye Pork Pie, Smash Palace und Utu (Introduction, 2008: 4-6). Mit dem Film Sleeping Dogs sieht ein großer Teil der Neuseeländer zum ersten Mal statt der früher gewohnten „Hollywoodkost“ einen Film mit spezifisch neuseeländischem Inhalt im Kino, was durchaus als Renationalisierung eines bisher kaum existenten neuseeländischen Kinos bezeichnet werden kann - sowohl auf der Produktions- als auch auf der Inhaltsebene. Der von Neuseeländern produzierte Film vermittelt ein selbstkritisches Bild von Neuseeland, in dem der offizielle Nationalismus einer konservativen, repressiven Regierung mit dem freiheitsliebenden, anti-autoritären Patriotismus des Protagonisten Smith kontrastiert wird - einer explizit nationalpolitischen Thematik, die bis dahin noch von keinem neuseeländischen Film thematisiert worden war.

Bei der Einführung sowie Beendigung der steuerlichen Vorteile für Filmproduktionen im Zeitraum 198I-1984 kam es - wie in der Analyse weiter oben bereits erwähnt - zu einer simultanen Re-sowie Denationalisierung des neuseeländischen Kinos durch die zum einen deutlich gestiegene Anzahl jährlich produzierter Filme, zum anderen aber damit zumeist einhergegangenen stärker transnationalen Produktionshintergründe dieser Filme. Hier könnte man auch von einer partiellen Denationalisierung der Filminhalte sprechen, da in diesem Zeitraum erschienene Filme wie Battletruck, Dead Kids, Death Warmed Up, Savage Islands und Merry Christmas, Mr. Lawrence entweder überhaupt keine oder nur schwach erkennbare neuseelandspezifische Thematiken aufgreifen. So können beispielsweise Dead Kids und Death Warmed Up als generische Horrorfilme eingestuft werden, die relativ eindeutig amerikanischen Vorbildern nacheifern, um damit ein größeres (internationales) Publikum anzusprechen und trotz ihrer Horrorthematik keine neuseelandspezifische Kiwi Gotbic-Ästhetik aufweisen. Allerdings sind im selben Zeitraum auch inhaltlich sehr neuseelandspezifische Filme wie Bad Blood, Trial Run und Pallet on the Floor erschienen, sodass nicht von einer generellen, sondern, wie erwähnt, vielmehr partiellen Denationalisierung der Inhaltsebene gesprochen werden sollte.

Während die neuseeländische Filmindustrie Jahre brauchte, um sich von der Steuerreform und der generellen Deregulierung der Wirtschaft in Neuseeland zu erholen, erschienen Anfang der 
I99oer schließlich Filme wie The Piano, Once Were Warriors und Heavenly Creatures, die trotz oder gerade aufgrund - der Neuseeländischkeit ihrer Inhalte ein weit über die nationalen Grenzen hinaus reichendes Filmpublikum erreichten. Diese Phase, die Conrich/Murray als second wave bezeichnen (Introduction, 2008: 7), könnte wiederum als Renationalisierung der Inhaltsebene gewertet werden, da die genannten Filme einen verstärkten Fokus auf neuseelandspezifische Inhalte aufweisen und diese Inhalte auch zum ersten Mal von einem globalen Publikum ernsthaft betrachtet und wertgeschätzt wurden. Hierbei ist allerdings anzumerken, dass diese Filme ein sehr hohes Produktionsniveau aufweisen, das von bisherigen neuseeländischen Filmen nur selten erreicht wurde. Nicht zuletzt durch die hollywoodähnliche Ästhetik dieser Filme konnte ein breites Publikum angesprochen werden. Insofern ist diese inhaltliche Renationalisierung mittels transnationaler Ästhetikstandards erzeugt worden.

Während die New Zealand Film Commission seit ihren Anfängen einen neuseelandspezifischen Inhalt als wichtiges Kriterium zur Förderung von Filmprojekten betrachtete und auch sehr erfolgreiche Filme wie Once Were Warriors mitfinanzierte, musste auch sie letztlich Kompromisse eingehen, weshalb die Förderungspraxis wiederholt kritisiert worden ist. So schreibt Conrich über die Zwiegespaltenheit der neuseeländischen Filmindustrie und der NZFC, die gerade in den letzten Jahren durch zunehmende Globalisierung verschärft wurde: "The hitherto small New Zealand film industry has been looking for ways to position itself in a sustainable 'niche' within the global market, with stories that are local yet appeal to worldwide audiences” (Hardy 2008: 130). Dieser Spagat zwischen kommerziellem Erfolgspotenzial und der Vermittlung nationalspezifischer Thematiken ist nicht immer geglückt. So konstatiert Murray, dass der Zwang zu kommerziellem Erfolg die Sichtweise auf das neuseeländische Kino als primär nationales Projekt der Selbstdefinition weiter geschwächt hat: "The transnational and increasingly globalised nature of filmmaking in New Zealand in the 1990's diluted the sense that a small, national creative community was producing narratives by which it defined itself and its place in the world" (2008: 178).

Insgesamt ist letztendlich eine Doppelstrategie der neuseeländischen Filmindustrie zu erkennen: Sowohl Filme, die neuseelandspezifische Thematiken behandeln, als auch Filme, die bewusst auf eine solche Spezifizität verzichten und damit eine inhaltliche Denationalisierung darstellen, werden produziert. Zum einen kann der Verzicht auf nationalspezifische Aspekte in Filmen wie The Irrefutable Truth About Demons oder Perfect Creature durch besonders einfache Zugänglichkeit ein globales Publikum wirksam ansprechen. Zum anderen kann aber auch gerade die Fo- 
kussierung auf nationalspezifische Aspekte einen Unique Selling Point darstellen, mit dem sich Filme wie The Piano und Whale Rider erfolgreich gegenüber dem Hollywood-Mainstream abheben können und durch ihren exotischen Appeal für Zuschauer interessant werden. Neuseelandspezifische Filminhalte haben somit ein ambivalentes Potenzial: Um ein transnationales Publikum erfolgreich anzusprechen, dürfen sie trotz ihrer Spezifizität auch nicht zu speziell sein, was erklärt, warum ein sehr spezieller Film wie Ngati trotz seiner Thematik, die viele Parallelen zu Whale Rider aufweist, global kaum rezipiert wurde und kommerziell erfolglos war.

\subsection{Ergebnis}

Während sich die De- und Renationalisierungsprozesse auf der inhaltlichen Ebene zwar wie erörtert grundsätzlich feststellen lassen, aber mangels empirischer Messbarkeit keine genauen Trends ermittelt werden können, lässt sich von der De- und Renationalisierung der Produktionsebene nun ein zeitlich akkurateres Bild zeichnen: Obwohl die De- und Renationalisierungsprozesse auf der Produktionsebene nicht linear ablaufen, sondern von Schwankungen geprägt sind, lassen sich Trends und Schwerpunkte in der Entwicklung erkennen. Bereits ab 198I mit dem Erscheinen des Films Bad Blood, d. h. vier Jahre nach den Anfängen des zeitgenössischen neuseeländischen Kinos, hat es Produktionen deutlich transnationalen Charakters gegeben, die aufgrund ihres national hybriden Charakters eine eindeutige Klassifizierung als „neuseeländisch“ oder „ausländisch“ erschwerten und hierdurch den Diskurs um das nationale neuseeländische Kino problematisiert haben. So hat die Konsens-Dissens-Analyse gezeigt, dass es einen relativ hohen Dissens über $8 \%$ der Filme bezüglich ihrer Klassifikation als „neuseeländisch“ oder „ausländisch“ gibt.

Im zeitlichen Verlauf hat sich gezeigt, dass die Zunahme/Abnahme der Anzahl der produzierten Filme mit der Verringerung/Steigerung der (subjektiv empfundenen) „,nationalen Homogenität“ der einzelnen Filme in den Filmkatalogen korreliert, da eine erhöhte Anzahl produzierter Filme in einem Jahr praktisch immer mit einer gesteigerten Anzahl von Koproduktionen einhergeht. Die zwei wichtigsten Kräfte bzw. Auslöser, die das Wechselspiel zwischen de- und renationalisierenden Entwicklungen bestimmen, sind die Steuerpolitik Neuseelands und die Förderpraxis der NZFC. Während das „Steuerloch“ in den 1980ern und der Large Budget Screen Production Grant in quantitativer Hinsicht zeitweise zu einer Renationalisierung geführt haben, da mehr produzierte Filme pro Jahr auch eine stärkere Präsenz des neuseeländischen Kinos bedeutet, hat sich in qualitativer Hinsicht durch die damit einhergehende vermehrte Involvierung ausländischer Pro- 
duktionsfaktoren die „nationale Reinheit“ der einzelnen Filme verringert, was als Denationalisierung gewertet werden kann. Die NZFC hingegen hat allein das Fördern von Filmen mit „specific New Zealand content" zum Ziel gehabt, was eine den genannten denationalisierenden Faktoren entgegensteuernde Renationalisierungsmaßnahme darstellt. Dass trotz verstärkt transnationaler Produktionshintergründe vieler Filme diese dennoch als „,neuseeländisch“ eingestuft werden, illustriert, wie die subjektiven Zuordnungskriterien der Filmkatalogersteller sich „gelockert“ haben bzw. „flexibler“ geworden sind: Eine weniger strenge Auslegung dessen, was einen „specific New Zealand content" ausmacht, ist in Zeiten verstärkter Globalisierung eine Notwendigkeit geworden, um weiterhin überhaupt noch eine nennenswerte Anzahl von Filmen dem Filmkorpus des nationalen neuseeländischen Kinos zuordnen zu können. Diese Zuordnungsstrategie könnte ebenfalls als Renationalisierung bezeichnet werden, da hierdurch versucht wird, die „Legitimität“ des Konstrukts des nationalen Kinos weiterhin aufrechtzuerhalten.

Neben der bis jetzt angeführten subjektiven De- und Renationalisierung lassen sich auf der objektiv messbaren Ebene folgende Ergebnisse zusammenfassen: Hinsichtlich der Involvierung neuseeländischer Produktionsfirmen im neuseeländischen Kino lässt sich seit den Anfängen des zeitgenössischen neuseeländischen Kinos insgesamt eine partielle Denationalisierung beobachten. Die Denationalisierung dieses Bereichs ist daher kein wirklich neuer Trend, da es Koproduktionen ebenfalls bereits früh gegeben hat. Dies lässt sich damit erklären, dass das zeitgenössische neuseeländische Kino ohnehin im Vergleich zum Kino anderer Länder sehr jung ist, da erst 1977 mit Sleeping Dogs ihr Beginn als zeitgenössisches Kino eingeleitet wurde und deshalb die Globalisierung in ihrer modernen Form hier unmittelbar einwirken konnte. Nur bezüglich der Produktionsfirmen nationaler Minderheiten lässt sich ein leicht positiver Trend erkennen.

Bezüglich der Unterebene „Regisseur“ lässt sich hingegen insgesamt nur eine geringfügige Veränderung feststellen, was sich auch darauf zurückführen lässt, dass in der Filmproduktion traditionell nur ein Regisseur mitwirkt und es deshalb praktisch keine neuseeländisch-ausländische $\mathrm{Zu}$ sammenarbeit in dieser Unterebene gibt. Diese Unterebene zeigt sich demnach gegenüber Globalisierungseinflüssen als relativ resistent - die des Öfteren von Neuseeländern hervorgebrachte Klage eines „cinematic brain drain“ erscheint deshalb übertrieben und mehr auf subjektiven Empfindungen als auf Fakten zu beruhen. Die Involvierung nationaler Minderheiten als Regisseure ist signifikant, wobei sich erst $\mathrm{ab} 2000$ ein eindeutig positiver Trend und $\mathrm{ab} 2003$ außerdem eine ethnische 
Diversifizierung (neben Produktionen von Māori nun z. B. auch von Samoanern, Fidschianerin und Indern) verzeichnen lässt.

Dass sich hingegen bei den Drehbuchautoren die Anzahl ausländischer Drehbuchautoren zulasten neuseeländisch-ausländischer Kollaborationen und rein neuseeländischer Skripte erhöht hat, lässt sich unter anderem darauf zurückführen, dass es - zumindest aus der Sicht von Filmproduzenten wie Peter Jackson - in Neuseeland kaum talentierte Drehbuchautoren gibt. Statt das Drehbuch eines neuseeländischen Autors von einem professionellen Skriptwriter aus Hollywood überarbeiten zu lassen, wird stattdessen aus pragmatischen Gründen das Verfassen des Drehbuches immer öfter von vornherein ins Ausland verlagert. Diese Entwicklung lässt sich dadurch erklären, dass erfahrene Skriptwriter aus Hollywood einen Vorsprung gegenüber weniger erfahrenen neuseeländischen Drehbuchautoren haben, wenn es um das Verfassen von Skripten geht, die nicht Ausdruck nationalspezifischer „Befindlichkeiten“ sind, sondern gezielt ein globales Publikum ansprechen sollen - eine Anforderung, die immer wichtiger zu werden scheint. Ähnlich wie bei den Regisseuren lässt sich auch bei den Drehbuchautoren eine steigende Involvierung von Minderheiten verzeichnen. Der im Vergleich zu den Regisseuren höhere Anteil von Minderheiten lässt sich darauf zurückführen, dass nationale Minderheiten bei Filmen über Minderheitsthematiken zuerst bei der Drehbuchproduktion mitgewirkt haben, bevor sie schließlich auch als Regisseure tätig wurden.

Zusammenfassend bedeutet dies, dass der Sektor „location“ in der Gesamtsicht am wenigsten, der Sektor „Produktionsfirma“ hingegen im Schnitt am stärksten von Denationalisierungsprozessen betroffen ist. Zwar ist der Entwicklungstrend in allen Fällen recht gering und liegt bei $-2 \%$ bis $-4 \%$, allerdings gibt es im Ausgangsniveau, wie bereits angemerkt, beträchtliche Unterschiede. Aufgrund der subjektiven Beurteilung des Sektors „Produktionsfirma“ als am wenigsten relevant, gab es also, wie die Konsens-Dissens-Analyse gezeigt hat, am wenigsten Bedenken, einen Film mit signifikanter Beteiligung ausländischer Produktionsfirmen in den Katalog neuseeländischer Filme aufzunehmen, wohingegen eine ausländische location am ehesten zu einem Konsens bezüglich seines Ausschlusses geführt hat. Mit anderen Worten ist der Sektor „Produktionsfirma“ am stärksten transnational ausgerichtet, wohingegen die Sektoren „Regisseur“ und „Drehbuchautor" stark national und der Sektor „location“ am stärksten national ausgerichtet sind.

Hinsichtlich der Beteiligung nationaler Minderheiten an neuseeländischen Filmproduktionen lässt sich abschließend sagen, dass ihre Involvierung in den Produktionsprozessen bis 2002 nur 
vereinzelt gegeben war, seitdem aber deutlich regelmäßiger geworden ist, wenn auch der Gesamttrend nur eine leichte bis mittlere Steigerung andeutet. Ihre Mitwirkung am vorwiegend ökonomischen Sektor „Produktionsfirma“ ist weiterhin als gering einzustufen, wohingegen ihre Mitwirkung als Regisseur und Drehbuchautor, d.h. den „kreativeren“ Sektoren deutlich stärker geworden ist. Während die indigene Māori-Bevölkerung den größten Anteil an den Filmproduktionen hat, ist ebenfalls seit 2002 eine verstärkte Involvierung nichtindigener nationaler Minderheiten wie Indern, Chinesen und Samoanern zu verzeichnen.

In objektiver Hinsicht ist die Verdrängung neuseeländischer locations durch ausländische locations sehr gering, wobei der Anteil neuseeländischer locations sich mit rund $90 \%$ ohnehin bereits auf einem sehr hohen Niveau bewegt. Dies spiegelt zum einen den Umstand wider, dass nahezu alle stark national ausgerichteten Produktionen auch in Neuseeland gedreht werden, und zum anderen, dass auch lediglich partiell in Neuseeland gedrehte stärker transnational ausgerichtete Produktionen mit hoher Regelmäßigkeit wegen des Drehs in Neuseeland bereits als neuseeländische Filme eingestuft werden.

Die Auswertung der Kriterienmatrix hat gezeigt, dass generell die Produktionsebene eine größere Rolle als der Filmtext bei der Bewertung eines Films als „neuseeländisch“ oder „ausländisch“ spielt. Mit anderen Worten werden Filme, die weder einen neuseeländischen Handlungsort noch eine Filmhandlung aufweisen, in der spezifisch neuseeländische Thematiken behandelt werden, dennoch regelmäßig als neuseeländische Filme eingestuft, wenn auf der Produktionsebene genügend Unterebenen neuseeländisch sind. Bezüglich des Verhältnisses der einzelnen Unterebenen zueinander lässt sich folgende Hierarchie feststellen: Weder ein Einzelkriterium, noch eine Zweier-Konstellation, noch die meisten Dreier-Konstellationen sind ausreichend, um einen vollen Konsens bezüglich der „Neuseeländischkeit“ eines Films zu erreichen. Erst die Vierer-Konstellation, bei der auf allen Unterebenen neuseeländische „Elemente“ mitgewirkt haben, bewirkt einen vollen Konsens und ist mit einer Häufigkeit von fast $80 \%$ als wichtigste Konstellation einzustufen. Bei der Einstufung eines Films als neuseeländisch oder ausländisch ist isoliert betrachtet das Kriterium „location“ das ausschlaggebendste, evtl. gefolgt von den Kriterien „Prestige“ und „finanziellem Erfolg“, die im Zweifelsfall einen Teil der restlichen Kriterien „Regisseur“, „Drehbuchautor“, „Produktionsfirma“ und auch „Handlungsort“" verdrängen können.

Erstaunlicherweise spielt somit die location bei der Einstufung eines Filmes eine wesentlich größere Rolle als die Produktionsfirma. Dieser Umstand lässt sich unter anderem damit erklären, 
dass die neuseeländische Landschaft bereits seit den Anfängen der Kolonisation eine wichtige Rolle im nation building-Prozess und in der nationalen Identität Neuseelands gespielt hat und deswegen auch im Diskurs um ein nationales neuseeländisches Kino die Klassifikation eines Films als „neuseeländisch“ ohne eine neuseeländische location (als geografisches „Fundament“ des Films) kaum zulässt. Da die neuseeländische Landschaft von Neuseeländern als einzigartig und als wichtiges Unterscheidungsmerkmal zu anderen Ländern herangezogen wird, kann ein Film gewissermaßen nur „wirklich neuseeländisch“ sein, wenn auch eine neuseeländische location zum Einsatz kommt. Selbst von der globalen Zuschauerschaft wird ein Film dann am stärksten mit Neuseeland assoziiert, wenn er dort gedreht wurde. Dies ist auch dann der Fall, wenn der Handlungsort nichtneuseeländisch ist, wie vor allem die Lord of the Rings- und die Hobbit-Trilogie und der durch sie ausgelöste Filmtourismus nach Neuseeland eindrücklich belegen.

Grundsätzlich betrachtet ist der „nationale Film“ letztlich ebenso wie „nationale Literatur“ und „nationale Kunst“ ein Medium, über das sich eine Nation in ihren multiplen nationalen Identitäten definieren, bestätigen oder auch infrage stellen kann. Bei der Definition dieser Medien als „national“ wird nicht allein auf die Nationalspezifizität des Inhalts und der Form abgestellt, sondern auch auf die Nationalität der Produzenten dieser Filme, Bücher etc. Nationen geben sich also auch als „Produzenten“ dieser Medienformen aus, analog zu Autos „made in Germany“ also auch Filme „made in New Zealand“, was zugleich eine Vermarktungsstrategie und Aushandlung der eigenen Identität darstellt. Wenn auf den Produktionsstandort fokussiert wird, dann wird hierbei in räumlichen Kategorien gedacht, die letztlich noch wichtiger zu sein scheinen als der zeitliche Aspekt: Wichtig ist weniger, wann ein Film produziert wurde, sondern vor allem, wo er produziert wurde. Das „Wo“ (Handlungsort des Films, location, Produktionsstandort) definiert das Nationale eines Films also stärker als das „Wann“.

Was hierbei im Rahmen des ersten Kapitels nicht beantwortet werden konnte, ist, warum überhaupt neuseeländische locations auch in Filmen eingesetzt werden, die inhaltlich keine neuseeländischen Themen behandeln. Da in solchen Filmen folglich der Handlungsort nicht-neuseeländisch ist, wird hier gewissermaßen die „Neuseeländischkeit“ der neuseeländischen location geleugnet und verschleiert - und zwar nicht nur auf inhaltlicher Ebene, sondern auch auf ästhetischer, wobei auch vermehrt digitale Effekte eingesetzt werden und dadurch die Komplexität der Filmgeografie solcher Filme durch Einbeziehung virtueller - und damit gewissermaßen „anationaler“ Artefakte - noch gesteigert wird. Aufgrund der großen Bedeutung der location und deren Wir- 
kung als im Hintergrund wirkende filmgeografische und ästhetische Instanz, soll das Verhältnis zwischen location und Handlungsort nun im nächsten Kapitel thematisiert werden. 


\section{De-/Entlokalisierung der neuseeländischen location und dessen Bedeutung} für die Frage nach filmgeografischer Authentizität und Filmtourismus

\section{I Einleitung}

In Kapitel I wurde die De- und Renationalisierung des nationalen neuseeländischen Kinos erörtert und gezeigt, dass die location eine wichtige Rolle bei der Konstruktion des neuseeländischen Kinos spielt. Hierbei werden neuseeländische locations keineswegs nur bei Filmen eingesetzt, deren Handlung sich in Neuseeland abspielt: Neuseeland hat sich mittlerweile auch einen Namen als beliebte location für Hollywood-runaways gemacht, deren Handlungsorte praktisch immer nicht-neuseeländisch sind. ${ }^{29}$ Die Bandbreite der Handlungsorte, die durch neuseeländische locations simuliert werden, reicht von auf realen Orten/Regionen basierenden Handlungsorten wie Oregon in Without a Paddle bis hin zu rein fiktiven Handlungsorten wie "Mittelerde" in der Lord of the Rings-Trilogie.

Wenn die neuseeländische location derart wichtig bei der Konstruktion eines nationalen neuseeländischen Kinos ist, dann erscheint es interessant, Funktion und ästhetische Wirkung der neuseeländischen location in diesen Filmen zu analysieren. Das primäre Interesse gilt hier den formalästhetischen Aspekten der Filme, wozu auch die Produktionshintergründe und Zuschauerrezensionen beleuchtet werden sollen, wohingegen die narrativische Ebene der Filme eine nur untergeordnete Rolle bei der Analyse spielen wird..$^{30}$ Der Fokus soll hierbei auf die runaways gerichtet werden, da gerade diese Filme aufgrund ihrer grundlegenden filmgeografischen „Inkongruenz" die interessantesten Fragen aufwerfen. Durch die Kombination neuseeländische location/nichtneuseeländischer Handlungsort stellt sich die Frage nach filmgeografischer „Authentizität" und damit in Zusammenhang stehenden filmtouristischen Aspekten: Sehen sich Filmemacher veranlasst, ihre Entscheidung zu rechtfertigen, beispielsweise bei der Produktion von Without a Paddle in Neuseeland statt in Oregon gedreht zu haben, obwohl die Handlung sich nur in Oregon (und nicht in Neuseeland) abspielt (= Authentisierungsstrategien)? Wird das geografische „Auseinanderdriften" von location und Handlungsort von Zuschauern erkannt und beeinträchtigt das in ihren Augen die (geografische) Glaubwürdigkeit des Films (= Authentizitätserwartungen)? Inwie-

\footnotetext{
${ }^{29}$ Mit Ausnahme von Green Dolphin Street (1947) ist mir kein Hollywoodfilm bekannt, dessen Handlungsort Neuseeland gewesen wäre. Während Handlungsorte wie USA, der nahe Osten, verschiedene afrikanische Länder und europäische Länder wie Frankreich sehr häufig dargestellt werden, stellt Neuseeland quasi einen „weißen Fleck“ auf der „Weltkarte“ der Handlungsorte des Hollywood-Kinos dar.

${ }^{30}$ Da die narrativische Ebene von Filmen, deren Handlungsort Neuseeland ist, aber durchaus interessante Fragen aufwirft, werde ich hierzu in Punkt 3.3 einige Überlegungen anstellen.
} 
fern beeinträchtigt ein als unglaubwürdig bewerteter Film dessen filmtouristisches Potenzial? Welche Rolle spielen digitale Effekte bei der Konstruktion „authentischer" Handlungsorte; inwiefern können sie die Glaubwürdigkeit des Handlungsortes steigern oder verringern? Und was passiert, wenn reale locations durch digitale Effekte bzw. sogenannter computer-generated imagery (CGI) immer stärker „maskiert" oder gar vollständig ersetzt werden? Kann ein Film, der nicht on location, sondern komplett im Filmstudio mit digital generierten Landschaften produziert wurde, noch Filmtourismus auslösen und was sind die Voraussetzungen hierfür?

Diesen Fragestellungen soll in diesem Kapitel nachgegangen werden, wobei die grundsätzliche Problematik in diesen Filmen darin zu sehen ist, dass es durch die Darstellung nicht-neuseeländischer Handlungsorte mittels neuseeländischer locations zu einem filmgeografischen „Aneinanderreiben" zweier eigentlich inkompatibler Geografien kommt.

Hierzu soll folgendes Filmkorpus analysiert werden (Auflistung in chronologischer Reihenfolge):

\begin{tabular}{|l|}
\hline 1988 Willow (Handlungsort: nicht näher benannte fiktive Fantasywelt) \\
\hline 1999 Vertical Limit (Handlungsort: Karakorum-Gebirge) \\
\hline $\begin{array}{l}\text { 200I-2003 Lord of the Rings: The Fellowship of the Ring, The Two Towers, The Return of the King (Hand- } \\
\text { lungsort: fiktives Mittelerde) }\end{array}$ \\
\hline 2003 Last Samurai (Handlungsort: historisches Japan) \\
\hline 2004 Without a Paddle (Handlungsort: Oregon) \\
\hline 2005 King Kong (Handlungsort: fiktives Skull Island und New York) \\
\hline 2007 The Waterhorse: Legend of the Deep(Handlungsort: Schottland, insb. Loch Ness) \\
\hline 2009 Avatar (Handlungsort: fiktives Pandora) \\
\hline $\begin{array}{l}\text { 20IO-2013 The Hobbit: An Unexpected Journey, The Desolation of Smang, The Battle of the Five Armies } \\
\text { (Handlungsort: fiktives Mittelerde) }\end{array}$ \\
\hline 20II Good for Nothing (Handlungsort: historische USA [„Wilder Westen“]) \\
\hline
\end{tabular}

Die aufgelisteten Filme können in verschiedene Handlungsort/location-Konstellationen kategorisiert werden, die später im Methodikteil unter Zuhilfenahme von Baudrillards Simulationstheorien als „Simulationsstufen” weiter ausdifferenziert werden sollen und die Grundlage für die zu formulierenden Fragestellungen und Thesen bilden werden: 
I. Konstellation: zeitgenössischer, realitätsbasierter, nicht-neuseeländischer Handlungsort/neuseeländische location

- Vertical Limit

- Without a Paddle

- The Waterhorse: Legend of the Deep

2. Konstellation: historischer, realitätsbasierter, nicht-neuseeländischer Handlungsort/neuseeländische location

- Last Samurai

- Good for Notbing

3. Konstellation: fiktiver Handlungsort/neuseeländische location

- Willow

- Lord of the Rings

- The Hobbit

4. Konstellation: fiktiver Handlungsort/keine location (digital generierte Landschaft)

- King Kong

- Avatar

Die Konstellationsgruppen I und 2 unterscheiden sich in erster Linie durch ihre unterschiedlichen zeitlichen Bezüge. Während sich bei der I. Konstellation primär eine geografische Diskrepanz zwischen Handlungsort und location ergibt, unterscheiden sich bei der 2. Konstellation Handlungsort und location zusätzlich auch in zeitlicher Hinsicht, was den Produktionsaufwand für die Filmemacher erhöht, da hierbei eine historische, also in der Form nicht mehr existente Landschaft rekonstruiert werden muss. So muss beispielsweise bei Last Samurai die neuseeländische location nicht nur ,japanisiert“, sondern zusätzlich auch „historisiert“ werden, um von den $\mathrm{Zu}$ schauern als glaubhafte Darstellung eines historischen Japans akzeptiert zu werden. Die Inkongruenz zwischen Handlungsort und location versuchen Filmemacher in der Regel zu verschleiern, indem sie durch gezieltes Entfernen und Platzieren bestimmter „Landschaftsmarker“ den Eindruck suggerieren, als sei tatsächlich dort gedreht worden, wo der Handlungsort vorgibt zu sein. Diese 
Strategie einer „filmgeografischen Delokalisierung“, die Lukinbeal/Zimmermann als „crimes against geography“ (2006: 319) und Beeton als einen Fall von „mistaken identities“ (2005: I0, I55) bezeichnen, führt bei Zuschauern zu einem regen Diskurs im Internet (z. B. Internet Movie Database) über die filmgeografischen Darstellungsweisen und die Glaubwürdigkeit solcher Filme, worauf in der Analyse genauer eingegangen wird.

Die zentralen Fragestellungen sind:

I. Welche filmgeografischen Konstruktions- und Authentisierungsstrategien setzen Filmemacher ein, um die grundlegende „filmgeografische Inkongruenz“ zu kompensieren und den filmischen Handlungsort (geografisch) glaubwürdig wirken zu lassen?

2. Werden diese Strategien von den Zuschauern akzeptiert und von welchen Bedingungen ist dies abhängig?

3. Inwiefern beeinflusst der Grad filmgeografischer „Authentizität“ das filmtouristische Potenzial dieser Filme?

Während bei den Konstellationsgruppen I und 2 ein doppelter Bezug zu realen Geografien gegeben ist, weil Zuschauer sowohl den Handlungsort als auch die location mit realen Orten assoziieren können, ist bei den Konstellationsgruppen 3 und 4 dieser Realitätsbezug verringert oder ganz verschwunden. Obwohl beispielsweise der Handlungsort „Mittelerde“ fiktiv ist und somit von Filmtouristen nicht besucht werden kann, konnte die Lord of the Rings-Trilogie dennoch aufgrund des Einsatzes neuseeländischer - d. h. realer - locations Filmtourismus erzeugen, da Filmtouristen Neuseeland gewissermaßen als die physische „Realisierung“ Mittelerdes behandelten. Dies ist jedoch bereits eine Einschränkung im Vergleich zu einem Film der I. Konstellation wie beispielsweise The Waterhorse: Legend of the Deep, wo der Zuschauer prinzipiell die Wahl hat, entweder den vom Film dargestellten Handlungsort Schottland zu besuchen oder aber Neuseeland, wo der Film tatsächlich gedreht wurde. Bei Filmen der 4. Konstellation schließlich gibt es grundsätzlich gar keinen konkreten Bezug zu realen Geografien mehr. So hat beispielsweise King Kong keinerlei Filmtourismus ausgelöst, weil der Handlungsort „Skull Island“ fiktiv ist und außerdem nicht etwa auf neuseeländischen locations gedreht, sondern in einem neuseeländischen Filmstudio digital generiert wurde.

Trotz ihres schwachen oder fehlenden Realitätsbezuges werden allerdings auch fiktive Welten von Zuschauern als authentisch oder inauthentisch beurteilt, wobei hier sowohl die neuseeländische location als auch die digitalen Effekte bzw. CGI eine wichtige Rolle spielen. Konkret sollen 
für die Filme der 3. und 4. Konstellationsgruppe zusätzlich zu den bereits für die I. und 2. Konstellationsgruppe formulierten Fragen folgende Fragestellungen erörtert werden:

I. Welche pragmatischen und vor allem ästhetischen Gründe gibt es für den vermehrten Einsatz von CGI in Fantasyfilmen?

2. Ist der Einsatz von CGI aufgrund moderner Sehkonventionen womöglich sogar unabdingbar, damit die dargestellten Fantasywelten von Zuschauern als „authentisch“ und immersiv wahrgenommen werden?

3. Welche Auswirkungen hat die zunehmende digitale Manipulation von locations sowie deren Ersetzung durch digitale Landschaften auf Neuseeland als location, Produktionsstandort und filmtouristisches Reiseziel?

4. Können fiktive digitale Landschaften von Zuschauern noch als in irgendeiner Form referenziell auf reale Geografien verweisend interpretiert werden? Mit anderen Worten, können Fantasyfilme, die auf rein digitalen, d. h. virtuellen „locations“ beruhen, überhaupt noch Filmtourismus generieren?

Im folgenden Methodikteil soll die bis jetzt vorgenommene Einteilung in verschiedene Handlungsort/location-Konstellationen in ein systematisches, nach bildsemiotischen Ansätzen konzipiertes Simulationsstufenmodell überführt werden, in Anlehnung an Baudrillards Theorien zur Simulation. 


\subsection{Methodik}

\subsection{Simulationsstufenmodell}

Im Folgenden soll ein Simulationsstufenmodell entwickelt werden, das Jean Baudrillards Theorien über Simulation, Simulakrum und Hyperrealität als Ausgangspunkt nimmt und die strukturelle Grundlage dieses Kapitels bilden wird. Als Antwort auf die grundlegende Frage, weshalb es Simulation überhaupt gebe und sie gerade in der Postmoderne rasant zugenommen habe, schreibt Baudrillard: „When the real is no longer what it used to be, nostalgia assumes its full meaning“" (198I: 17I). Er konstatiert somit, dass Simulation die Sehnsucht nach alternativen Realitäten ausdrücke und spricht in diesem Zusammenhang von einer „desert of the real“ (198I: 166). Gerade ab der Mitte des 20. Jahrhunderts sieht er eine nie da gewesene Dominanz der Simulakren, der „graue Alltag“ wird mit der nostalgischen Sehnsucht der Zuschauer nach einem Goldenen Zeitalter kontrastiert, die beispielsweise in Disneyland (oder filmspezifisch: vor allem in Fantasyfilmen) ihren Ausdruck findet (1981: 17I-172). Wenn Baudrillard demnach Simulation mit Nostalgie und Utopie in Verbindung bringt, dann impliziert er auch, dass die heutige westliche Gesellschaft von fundamentaler Unzufriedenheit gekennzeichnet ist und deshalb regelrecht in die Simulation flüchtet. Simulationsintensität und Unzufriedenheit scheinen sich hierbei gegenseitig zu verstärken: Je farbenfroher, „hyperrealer“ und larger than life die Simulationen, desto grauer erscheint der Alltag und desto stärker wird die Sehnsucht, diesem Alltag zu entfliehen.

Neben der Flucht in filmische Simulationen wird auch der Massentourismus als ebenfalls relativ modernes Phänomen als Möglichkeit betrachtet, diesem grauen Alltag durch das Erleben von Nicht-Alltäglichem zu entkommen (Urry 1990: I). In dem Erwecken der Sehnsucht nach „fernen, exotischen Ländern“, „Regeneration“ und „Anregung“ als Voraussetzung für tourism inducement spielen Filme eine wichtige Rolle, wie beispielsweise Beetons Film Induced Tourism, Roeschs The Experience of Film Location Tourists und Zimmermanns Wüsten, Palmen und Basare darlegen. Zimmermann merkt an, dass das Betrachten insbesondere landschaftsbetonter Filme für den Zuschauer zudem eine Art des virtuellen Reisens und eine Art Wissensquelle über die Geografie sowie den Charakter noch nie besuchter Länder oder Gebiete darstellt (2007: 25). Zusammen mit Tourismusbroschüren, touristischen Internetseiten, Reisereportagen und ggf. Nachrichten bildet sich der Zuschauer ein Bild von anderen Ländern, das mitunter stark verzerrt sein kann. Ähnlich wie sich der Tourist vom Reisen nicht-alltägliche Erlebnisse verspricht, erwarten auch Zuschauer, dass Filme ihnen nicht-alltägliche Erlebnisse bereiten, beispielsweise in Form spektakulärer Aben- 
teuer und Geschehnisse. Dies wird durch die ungebrochene Popularität actionlastiger Genres wie Adventure-, Science-Fiction- und Fantasyfilmen im Hollywoodkino bestätigt. Das Phänomen des virtuellen Reisens wird mitunter auch als armchair travel bezeichnet, wobei dieser Begriff eine leicht ironische Anspielung auf den eher passiven Charakter dieser „Reisen“ darstellt.

Hierbei zeigt sich auch eine grundsätzliche Assoziation von Simulation mit Inauthentizität, der das tatsächliche Bereisen eines Landes als höherwertiger und authentischer erachtete Aktivität gegenübergestellt wird. Somit kann das Phänomen des film tourism inducement als ein Prozess aufgefasst werden, der Zuschauer, die ein Land lediglich medial vermittelt erleben, in Touristen verwandelt, die das „echte“ Land vor Ort erkunden möchten, selbst wenn das filmisch konstruierte „Land“ fiktiv ist. Dabei wird offensichtlich, dass das Wissen über andere Nationen vor dem eigentlichen Bereisen praktisch ausschließlich medial, also in Form von Simulation, vermittelt wird. Baudrillards Aussage „the map [...] precedes the territory“ (198I: I66) reflektiert daher das Erlebnis der Filmtouristen: Zuerst sehen sie den Film, die virtuelle „Landkarte“, um anschließend das eigentliche „Territorium“ zu besuchen und die Simulation mit der Realität kognitiv und emotional abzugleichen. Lukinbeal betont die Bedeutung dieses Gedankens bei seiner Definition des Feldes der Filmgeografie: „Cinematic geography, or film geography is a growing transdisciplinary subfield that focuses on mapping the Baudrillardian terrain of cinema that precedes the cultural territory“ (2004: 248). Im Hinblick auf den Filmtourismus ist Boorstin bereits 1961 zu der Ansicht gekommen, dass es im Filmtourismus einen Paradigmenwandel gegeben habe, der mit der Vorrangstellung der Simulation vor der Realität einhergeht. Die hyperreale Landschaft in einem Film werde oft als das Ideal betrachtet, an dem die reale location von den Filmtouristen vor Ort gemessen werde, was einer Zelebrierung des „Inauthentischen“ gleichkomme: „We go not to test the image by the reality, but to test reality by the image“ (Boorstin 196I: II6). Dieser „Test“ wird aber durch den Einsatz fotorealistischer CGI sowie durch das geografische und zeitliche Auseinanderdriften von location und Handlungsort in vielen neueren Filmen zunehmend erschwert: Wenn beispielsweise in The Last Samurai sowohl japanische als auch neuseeländische locations den historischen japanischen Handlungsort simulieren und überdies auch CGI zum Einsatz kommt, kann der Zuschauer sich nicht mehr sicher sein, ob das, was er gerade sieht, Neuseeland, Japan, CGI oder auch eine nahtlose Zusammenführung dieser verschiedenen Geografien und Bildebenen zu einem homogenen Raum ist, was mit dem filmtechnischen Terminus des compositing beschrieben wird. Durch diese Pluralisierung der Zeichenbedeutungen taugt ein solcher Film aber immer weniger als geogra- 
fische Informationsquelle und kann von skeptisch gewordenen Zuschauern in filmgeografischer Hinsicht als inauthentisch eingestuft werden.

Baudrillard zufolge hat sich aber nicht nur die Quantität bzw. das Ausmaß der Simulakren verändert, sondern auch die Qualität der Simulation an sich, wobei sich die Zeichen immer stärker von der Realität loslösen. Hierbei sieht er eine chronologische Abfolge vier distinktiver Stadien:

These would be the successive phases of the image:

I It is the reflection of a basic reality.

2 It masks and perverts a basic reality.

3 It masks the absence of a basic reality.

4 It bears no relation to any reality whatever: it is its own pure simulacrum (1981: 170).

Baudrillard kommt zu dem radikalen Fazit, dass es zu einer vollständigen Sinnentleerung der Zeichen und einer völligen Referenzlosigkeit gekommen sei (170). Baudrillards geradezu apokalyptisches Szenario geht meines Ermessens zu weit, da die Vorstellung referenzloser Zeichen deren grundlegenden Verweisungscharakter und damit den Zeichenbegriff selbst obsolet machen würde. Die grundlegende Idee einer sukzessiven Verzerrung und Verringerung der Referenzialität von Zeichen erscheint mir allerdings sehr plausibel und soll als grundlegende Prämisse für das nachfolgend dargestellte Simulationsstufenmodell dienen. 


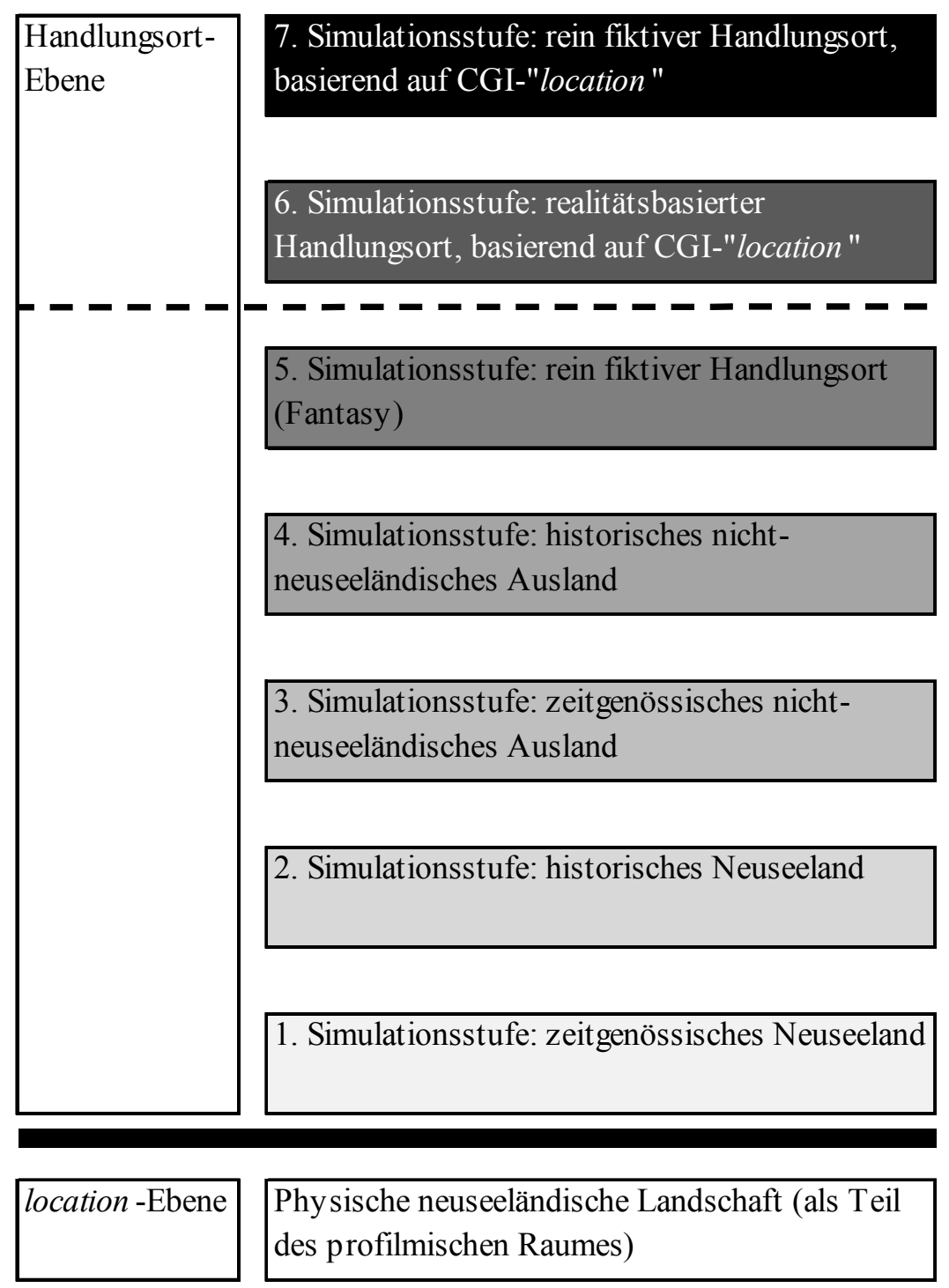

\section{Abbildung 3.I Simulationsstufenmodell}

Abbildung 3.I zeigt ein Simulationsstufenmodell, das verdeutlichen soll, wie sich mit steigendem Simulationsgrad die Simulation des Handlungsortes immer mehr von der physischen location, die die Grundlage und Projektionsfläche für die Simulation darstellt, entfernt - auf zeitlicher und auf geografischer Ebene. Während auf der I. Simulationsstufe das zeitgenössische Neuseeland als Handlungsort dient und daher die neuseeländische location in der Regel ohne großen Manipulationsaufwand „wie beschaffen“ abgefilmt werden kann, erfordert die Simulation eines historischen neuseeländischen Handlungsortes (2. Simulationsstufe) erheblich mehr Aufwand: ${ }^{31}$ Jegliche

\footnotetext{
${ }^{31}$ Ein Beispiel für einen Film der I. Simulationsstufe stellt der Film Whale Rider (2002) dar, wo ein nicht näher spezifiziertes Dorf an der Ostküste Neuseelands durch die geografisch identische neuseeländische location dargestellt wird. Aufgrund dieser hohen Ähnlichkeit können Filmtouristen die im Film gesehenen Landschaftsabschnitte beim Besuchen der location unmittelbar wiedererkennen. Das Piano (1993) stellt eines der bekanntesten Beispiele für einen Film der 2. Simulationsstufe dar, wo eine junge schottische Frau in den I85oern nach Neuseeland auswandert. Die historische Landschaft des Handlungsortes wurde unter anderem durch
} 
Artefakte, die die Jetztheit bzw. Verankerung der location im „Hier und Jetzt“ verraten, müssen entfernt sowie „historische“ Elemente platziert werden, um eine historische Landschaft (glaubwürdig) simulieren zu können. Das Übersehen oder bewusste Ignorieren moderner Artefakte, wie beispielsweise im Hintergrund fahrender Autos in einem Handlungsort, der das Neuseeland des 19. Jahrhunderts simulieren soll, wird als Filmfehler bzw. goof von Kritikern und insbesondere Zuschauern geahndet und in der Folge der Handlungsort als in seiner vorgeblichen Historizität wenig überzeugend bzw. inauthentisch wahrgenommen.

Während bis zu diesem Punkt nur die zeitliche Dimension betroffen ist, da in beiden Simulationsstufen der neuseeländische Handlungsort und die neuseeländische location geografisch grundsätzlich übereinstimmen, driften Handlungsort und location ab der 3. Simulationsstufe auch geografisch auseinander: Während bei einem Film wie Without a Paddle das zeitgenössische Oregon durch eine (zwangsläufig stets zeitgenössische) neuseeländische location simuliert wird und somit nur geografisch aber nicht zeitlich inkongruent ist, driften bei einem Film wie Last Samurai Handlungsort und location mehrfach auseinander (4. Simulationsstufe): Das historische Japan wird hier durch größtenteils neuseeländische locations simuliert, was bedeutet, dass Handlungsort und location sowohl geografisch als auch zeitlich nicht mehr übereinstimmen. Hierdurch wird die „Neuseeländischkeit“ der location geleugnet, was beispielsweise der Forderung der New Zealand Film Commission nach einem „specific New Zealand content“ in neuseeländischen Filmen teilweise widerspricht und als „Delokalisierung“ und auch Denationalisierung der location aufgefasst werden kann. Da in dem Film außerdem auch stellenweise japanische locations eingesetzt wurden und vereinzelt sogar Landschaftselemente neuseeländischer und japanischer locations digital zu einer homogen erscheinenden Filmlandschaft zusammengefügt wurden (compositing), kann sich der Zuschauer letztendlich nicht mehr sicher sein, ob er gerade eine japanische, eine neuseeländische oder ein location-composite im Film sieht. Mit anderen Worten hat sich hier die referenzielle Beziehung zwischen dem Handlungsort und der location pluralisiert und ist gleichzeitig destabilisiert worden. $^{32}$

Ein Film, dessen Setting einen rein fiktiven Handlungsort darstellt - sei er futuristisch, zeitgenössisch oder historisch - ist der 5. Simulationsstufe zuzurechnen: Hier ist der Handlungsort

den relativ unberührt wirkenden Kare Kare-Strand simuliert, wobei die an diesem Strand befindlichen Bungalows und Verkehrsschilder nicht gefilmt wurden, weil deren Modernität keine überzeugende Darstellung des vormodernen Handlungsortes ermöglicht hätte.

${ }^{32}$ Diese verminderte Referenzialität wirft sowohl Fragen der Authentizitätswertung als auch filmtouristische Fragen auf, die im weiteren Verlauf der Methodik (insbesondere Punkte 3.2.3.I und 3.2.3.4) wieder aufgegriffen werden. 
nicht mehr länger realitätsbasiert, sondern von jeglichem (real existierenden) nationalen Kontext gelöst und auf einer fiktiven Insel, in einem fiktiven Land oder in einer fiktiven Welt wie „Mittelerde" (Lord of the Rings-Trilogie) verortet. Dennoch ist auch die 5. Simulationsstufe indirekt in der Realität verankert, da sie eine real existierende location als Projektionsfläche nutzt, dessen Realität durch den „semiotischen Schleier“ des Handlungsortes kognitiv mehr oder weniger sichtbar bleibt. Dies erklärt auch den an sich erstaunlichen Umstand, dass Filmtouristen meinen, einem fiktiven Ort näherkommen zu können, indem sie die location, die als Projektionsfläche für den fiktiven Handlungsort gedient hat, besuchen. Der referenzielle Bezug ist hier in beide Richtungen gleichermaßen gegeben: Genauso wie Filmtouristen in der neuseeländischen location beispielsweise Mittelerde zu erblicken meinen, meinen Zuschauer im Handlungsort Mittelerde mehr oder weniger adäquate Informationen zur Geografie Neuseelands erblicken zu können. ${ }^{33}$ Dies zeigt auch, dass Referenzialität kein objektiver Bezug ist, sondern dass Zuschauer innerhalb eines gewissen Rahmens selbst entscheiden, welche locations sie als Referenten einstufen (und dann ggf. als Filmtouristen besuchen) möchten. Wie in Punkt 3.2.3.4 näher zu erläutern sein wird, verringert sich durch zunehmenden Einsatz von digital generierten Landschaftselementen der Grad der Referenzialität zur location, da diese durch die CGI-Elemente immer stärker maskiert wird und dadurch ein Filmtourist vor Ort mitunter kaum oder gar keine Ähnlichkeit mehr zwischen location und Handlungsort erkennen kann.

Dieser Prozess wird schließlich durch Filme, die praktisch ausschließlich auf digital generierten Landschaften beruhen, auf die Spitze getrieben. Filme wie King Kong, Tintin: Das Geheimnis der Einborn und Avatar benötigen überhaupt keine locations mehr, was hier als „Entlokalisierung" bezeichnet werden soll. Obwohl die Handlungsorte in diesen Filmen aufgrund der fotorealistischen Berechnung (rendering) durchaus „real“ wirken können, wurden sie praktisch komplett in einem Filmstudio gedreht. Trotz der im Vergleich zur 5. Simulationsstufe nochmals deutlich verringerten Referenzialität können allerdings auch hier je nach Art des Films Bezüge zu realen Geografien hergestellt werden. So ist das digitale New York in King Kong der 6. Simulationsstufe zuzurechnen, die semiotisch quasi die Umkehrung der 5. Simulationsstufe darstellt: Während bei der 5. Simulationsstufe der Handlungsort fiktiv, aber die location real ist, ist bei der 6. Simulationsstufe der Handlungsort realitätsbasiert, aber die „location “fiktiv bzw. virtuell: Den in der Anfangs-

\footnotetext{
${ }^{33}$ Dies setzt natürlich voraus, dass der Zuschauer Kenntnis von der neuseeländischen location hat, was auf die Bedeutung von Paraund Metatexten als Informationsquellen zu den in einem Film eingesetzten locations hinweist, die unter Punkt 3.2.3.3 besprochen werden und ebenfalls für Beurteilungen der „Authentizität“ eines Handlungsortes sowie für filmtouristische Handlungen eine wichtige Rolle spielen.
} 
szene von Tintin zu sehenden Place de Jeu de Balle im vollständig digital generierten Belgien kann der Zuschauer immer noch mit dem realen Belgien assoziieren, ebenso wie das digital generierte New York in der neuen King Kong-Verfilmung mit dem realen New York assoziiert werden kann. Daher können auch moderne Animationsfilme und auf digitalen Landschaften beruhende Filme trotz ihrer vollständigen Virtualität Filmtourismus auslösen. Im Falle von Finding Nemo (2003), in dem das Great Barrier Reef und Sydney virtuell nachgebildet wurden, hat die australische Tourismusindustrie sogar direkt mit diversen an den Film angelehnten Merchandisingartikeln und Gewinnspielen (u. a. einer Reise nach Australien) reagiert. Durch den immensen Erfolg ist der Film bereits als attraktivste Tourismuswerbung für Australien seit Crocodile Dundee (1986) bezeichnet worden (Beeton 2005: 65).

Den geringsten Bezug zu realen geografischen Räumen schließlich hat die siebte Simulationsstufe mit Filmen wie Avatar und der „Skull Island“-Sequenz in King Kong, bei denen der Handlungsort fiktiv und die „location“ virtuell ist. Diese Stufe kommt Baudrillards Verständnis von Simulation als einem Zustand, wo nur noch Selbstreferenzialität gegeben ist, am nächsten. Da aber auch hier noch oft genug bei der Generierung der digitalen Landschaften auf fotografische Texturen, d. h. auf „Fragmente“ realer Geografien zurückgegriffen wird, um nach Ansicht der Filmemacher einen höheren „Fotorealismus“ und damit „Authentizität“ der fiktiven Welt zu gewährleisten, kommt es auf den Einzelfall an, ob Zuschauer dennoch einen Bezug zu realen Geografien herstellen oder nicht.

Die Kategorisierung der Filme in die einzelnen Simulationsstufen ist nicht als starr zu betrachten, da es durchaus „Simulationsstufen-Hybride“ geben kann und die Übergänge zwischen den Simulationsstufen fließend sind. So wird in der Analyse von King Kong zu zeigen sein, dass der Film letztendlich ein Hybrid aus 6. und 7. Simulationsstufe darstellt und die Lord of the RingsTrilogie trotz ihrer Einordnung als Film der 5. Simulationsstufe auch Szenen enthält, die eindeutig der 7. Simulationsstufe zuzuordnen sind. Die letztendliche Einordnung eines Films in eine bestimmte Simulationsstufe ist quantitativ zu verstehen, d. h. ein Film, der immer noch in großem Umfang locations einsetzt, wird trotz gelegentlichen Einsatzes rein digitaler Landschaften dennoch der 5. Simulationsstufe zugeordnet, wohingegen ein Film wie King Kong trotz einiger weniger on location-Szenen der 7. Simulationsstufe zugeordnet wird, weil ein Großteil des Films auf digital generierten Landschaften beruht. 
Eine Sonderrolle spielen filmische Sets bzw. physische Modelle, die auf der location platziert werden: Da Sets in der Regel nur temporär sind und nach dem Filmdreh restlos aus der location entfernt werden, sind sie ebenso wie digitale Landschaften als Elemente der 7. Simulationsstufe einzuordnen. Der Unterschied zwischen physischen Modellen und CGI ist, dass die Modelle immerhin kurzzeitig im profilmischen Raum existiert haben, wohingegen CGI-Elemente nie physischer Teil der location gewesen sind und erst nach dem Dreh in der Postproduction-Phase eingefügt werden. Diese Differenzierung ist für den Filmtourismus indes irrelevant, da die Touristen weder die Modelle noch CGI vor Ort zu sehen bekommen werden. Permanente Sets wie „Hobbingen“ (Lord of the Rings) im neuseeländischen Matamata hingegen, die auch nach Abschluss des Filmdrehs erhalten bleiben, stellen eine dauerhafte physische Veränderung der location selbst dar und haben hohen filmtouristischen Wert. Da diese Sets somit Teil der Realität der location geworden sind, sind sie vielmehr der 5 . Simulationsstufe zuzuordnen.

\subsubsection{Authentizitätsbegriff und filmgeografische Authentizität}

Hinter der „Idee des Authentischen“ verbergen sich unter anderem Vorstellungen von Echtheit, Originalität und Wahrhaftigkeit (Daur 2013: I), die heutzutage auf eine Vielzahl von Bereichen angewendet werden und weit über die ursprüngliche Bedeutung des Begriffs hinausgehen: Auf Kunstauktionen ist der zweifelsfreie Nachweis der Urheberschaft von entscheidender Bedeutung - so wird ein als Fälschung und damit als inauthentisch erachteter „Van Gogh“ kaum einen Abnehmer finden und dieser „Kopie“ nur ein geringer Wert beigemessen werden. Auf psychologischer Ebene gelten Individuen als authentisch, wenn ihr Gefühlsleben im Einklang mit ihrem Verhalten steht. Auf medialer Ebene wird beispielsweise der „Echtheit“ von Nachrichten im Zeitalter praktisch beliebiger Manipulierbarkeit von Bildern eine zunehmende Skepsis entgegengebracht. Auch bei Spielfilmen, die geradezu zwangläufig von Inszeniertheit bestimmt sind, kann auf vielen Ebenen nach Authentizität gefragt werden, beispielsweise nach dem Grad der „Werktreue“ bei Literaturverfilmungen, der „Akkuratheit“ von Historienfilmen, aber auch nach der filmgeografischen Authentizität („Originalschauplätze“) sowie dem Grad an „Fotorealismus“ und Immersivität, wie später noch genauer zu erörtern sein wird.

Authentizität kann letztendlich als scheinbare Nicht-Inszeniertheit (Hattendorf 1999: 68) aufgefasst werden, dem negative Begriffe wie „künstlich“, „Fälschung“, „Kopie“ oder eben (offensichtliche) „Inszeniertheit“ gegenübergestellt werden. Während früher auch durchaus die Idee ob- 
jektiver Authentizität vertreten wurde, hat sich heute in der kulturwissenschaftlichen Forschung die Sichtweise durchgesetzt, dass Authentizität stets subjektiv und konstruiert ist. So schreibt Linder:

„Authentizität ist immer im Auge des Betrachters, ,etwas' ist immer für jemanden ,wahr'. Wenn uns ,Natürliches' als ,echt' erscheint, dann geht es nicht um die Frage, ob etwas, was dem Betrachter als ,natürlich' erscheint, auch ,wirklich' natürlich ist, sondern es geht um die Frage, warum dem Betrachter gerade ,Natürliches‘ als ,echt', als ,authentisch“ erscheint, warum ihm ,Natürliches' etwas ,bedeutet', ihm wichtig ist“"(1996: 80).

Aufgrund der Konstruiertheit von Authentizität argumentiert Regina Bendix, die Authentizität als einen grundlegenden Diskurs der Folklore Studies erachtet, dass nicht nach vermeintlich intrinsischen, „authentisierenden“ Qualitäten von Werken, sozialen Praktiken, Darstellungsweisen etc. zu fragen ist, sondern vielmehr nach den Motiven, Strategien und Erwartungen der Akteure, die den jeweiligen Authentizitätsdiskurs bestimmen: „The crucial questions to be answered are not , what is authenticity?' but ,who needs authenticity and why?' and how has authenticity been used?" (Bendix 1997: 2I).

Welche Akteure lassen sich im Diskurs um die Authentizität von Filmen ausmachen und in welchem Verhältnis stehen diese zueinander? Zur Beantwortung dieser Frage erweist sich Manfred Hattendorfs Definition von Authentizitätsdiskursen als Vertragsschluss zwischen verschiedenen Parteien als hilfreich (1999: 76-77). Hierbei stellt Hattendorf vor allem auf das juristische Prinzip von Leistung und Gegenleistung ab: Damit es zu einem Vertragsschluss kommt, muss beispielsweise bei einem Kaufvertrag der Verkäufer sein Verkaufsversprechen erfüllen und der Käufer die Ware abnehmen. Hierbei bedarf es besonderer Verkaufsstrategien, um den Käufer zum Kauf zu bewegen. Analog hierzu muss der von den Filmemachern produzierte Filmtext durch Einsatz bestimmter „Authentisierungsstrategien“ (1999: 77) die Authentizitätserwartungen der Zuschauer erfüllen, damit der Zuschauer das Filmprodukt als authentisch wahrnimmt bzw. die im Film vermittelten „Authentizitätssignale“ (1999: 72, 76) als überzeugend „abkauft“, d. h. als glaubwürdig wahrnimmt (1999: 19). Dieser „Wahrnehmungsvertrag filmischer Authentizität“ (1999: 76) wird nicht bewusst zwischen den Parteien geschlossen, sondern vielmehr konkludent, d.h. in stillschweigendem Einvernehmen (1999: 77). Sofern die Authentisierungsstrategien einen Zuschauer nicht überzeugen können, kann der Filmtext wiederum analog zum Kaufvertrag mit Sanktionen belegt werden: Wenn der Zuschauer das Filmprodukt als inauthentisch wahrnimmt, kann er diesen „Vertrags- 
bruch“ durch verschiedene Maßnahmen (negative Rezension, Boykott, etc.) ahnden. Welche Authentisierungsstrategien zum Erfolg führen, ist von den sich dynamisch verändernden, von soziokulturellen Faktoren beeinflussten Authentizitätserwartungen der Zuschauer abhängig. Die Authentizität eines Films ist also einem ständigen Aushandlungsprozess unterworfen: Ein Film, der vor zwanzig Jahren auf einer bestimmten Ebene als authentisch wahrgenommen wurde, kann heute als inauthentisch wahrgenommen werden.

Da Authentizität keine objektiv messbare, sondern eine subjektive Kategorie ist, kann die „Authentizität“ eines Films somit nur unter Berücksichtigung der Rezipientenseite umfassend erörtert werden, wozu sich eine semiotisch orientierte Herangehensweise empfiehlt. Insbesondere die semiotische Subdisziplin der Pragmatik, der „Erörterung des Zusammenhangs von Zeichen und Zeichenbenutzer sowie Text und Kontext" (Hattendorf 1999: I8) ist hier relevant, da sie der von Verfechtern einer Reader bzw. Viewer Response Theory geforderten Berücksichtigung der Rezipienten gerecht wird, statt sich einseitig auf den Filmtext zu beschränken. Obwohl Hattendorf eine - vorzugsweise empirisch angelegte - Analyse der Filmrezeption für wichtig hält, beschränkt er sich bei seiner Analyse von Authentizitätsdiskursen in Dokumentarfilmen auf den Filmtext und die Authentisierungsstrategien der Filmemacher, behandelt aber kaum die Zuschauerseite - mit der Begründung, dass dies den methodischen Rahmen seiner Arbeit sprengen würde (I999: I8, 68). Zwar kann die vorliegende Arbeit die Authentizitätserwartungen der Zuschauer ebenfalls nicht auf empirische Weise analysieren, da hierzu umfangreiche Zuschauerumfragen mit anschließender quantitativer Auswertung vonnöten wären. Stattdessen soll hier aber zumindest eine exemplarische Analyse von Zuschauerrezensionen (ggf. ergänzt durch die Analyse professioneller Rezensionen) geleistet werden, die bereits hilfreiche Rückschlüsse auf die Authentizitätserwartungen und -konzeptionen der Zuschauer zulässt. Die Rezeptionsanalyse soll die grundlegende formalästhetische Analyse der Filme ergänzen und dadurch eine möglichst umfassende Sicht auf die hier behandelten filmischen Authentizitätsdiskurse bieten.

Während bei Dokumentarfilmen die Authentizitätserwartung der Zuschauer sich darin begründet, dass das, was im Dokufilm zu sehen ist, sich „tatsächlich so ereignet“ hat, dass also „Schein“ und „Sein“ übereinstimmen und weder bewusst inszeniert, noch digital manipuliert worden ist, ist die Authentizitätserwartung bei Spielfilmen, die ja fiktional sind, anders gelagert: Hier geht es weniger darum, dass „Schein“ und „Sein“ übereinstimmen, sondern dass der „Schein“ so überzeugend vermittelt wird, dass er im Rahmen einer suspension of disbelief bereitwillig als mögli- 
ches „Sein“ akzeptiert wird. Mit anderen Worten muss die possible world, einem von Marie LaureRyan geprägten Begriff, überhaupt „possible“ im Rahmen der konventionalisierten internen Logik eines bestimmten Genres erscheinen. So wird im Fantasygenre Magie als „möglich“ eingestuft, wohingegen Zauberei in einem reinen Historienfilm als „unmöglich“ gilt und solch ein Film von einem Großteil der Zuschauer als unglaubwürdig eingestuft würde. Auch Eco kommt in seinen Ausführungen zum „Fiktionsvertrag“ zu dem Ergebnis, dass Leser (bzw. Zuschauer) ihre „Ungläubigkeit“ „nur in Bezug auf einige Dinge und nicht auf andere“ (1994: 106) aussetzen und nicht das Gefühl haben wollen, „belogen“ zu werden (1994: 103). Welche „Dinge“ dies für die hier zu analysierenden Simulationsstufen sind und welche Strategien die Filmemacher dabei einsetzen, soll im Folgenden genauer erörtert werden.

\subsubsection{Authentisierungsstrategien der Filmemacher und Authentizitätserwartungen der}

\section{Zuschauer}

3.2.3.I III. + IV. Simulationsstufe: Filmgeografische Delokalisierung als Authentisierungsstrategie

Die in einem Film dargestellte Geografie stellt einen wichtigen Faktor in der Konstruktion filmischer Realität dar. Escher/Zimmermann bezeichnen die Landschaft in Filmen als „Garant für Authentizität und Glaubwürdigkeit“, indem der Zuschauer den Ort als ihm tatsächlich bekannten oder als glaubwürdig umgesetzten Ort wahrnimmt und ihm dadurch „die Bedeutung eines lebensweltlichen oder zumindest der Geschichte dienlichem lebensweltlichen Ort“" zuweist (200I: 23I-232). Trotz aller Inszenierung hat ein Filmdreh an Originalschauplätzen durch seine als realistisch erachtete Abbildfunktion (Hattendorf 1999: 67) einen Informationswert, der über die eigentliche Inszenierung hinausgeht. ${ }^{34}$ Dies stellt einen der Gründe dar, warum die Blütezeit des Hollywood-Studiosystems mit seinen vorwiegend in Filmstudios (sogenannter Off location) produzierten Filmen schließlich von on location-Drehs, d. h. dem Dreh in offener Landschaft abgelöst wurde, da dies als realistischer und damit authentischer wahrgenommen wurde.

\footnotetext{
${ }^{34}$ Antonionis Film Blow Up (1966) thematisiert anschaulich die realistische Abbildfunktion der (damals noch analogen) Fotografie: Ein Fotograf macht Schnappschüsse von einem Liebespärchen in einem einsamen Londoner Park. Beim späteren Entwickeln und Vergrößern der Bilder meint er im Bildhintergrund eine Leiche zu erkennen. Tatsächlich findet er die Leiche bei einem nächtlichen Besuch des Parks vor. Die Fotos haben somit mehr von der Realität eingefangen als der Fotograf eigentlich inszenieren wollte, da das Ablichten der Leiche nicht Teil seiner künstlerischen Absicht gewesen ist. Auch Spielfilme, die früher gern als eine Form ,animierter Fotografie“ betrachtet wurden, tragen diesen Abbildcharakter in sich, da in einer Sekunde Film (üblicherweise) 24 „Fotografien“ geschossen werden.
} 
Hattendorf zeigt auf, wie bei dem 1946 erschienenen Spielfilm Paisa (1946), der von dem Schicksal verschiedener Personen im Italien zur Zeit des Zweiten Weltkriegs handelt, der Dreh an Originalschauplätzen als „Realitätsplus“ gewertet wurde und damit eine höhere „Authentizität des Ortes“ gewährleistete. Obwohl der Dreh an Originalschauplätzen zuerst eine Notlösung darstellte und eigentlich in Filmstudios gedreht werden sollte, bewertete die damalige Filmkritik den on location-Dreh als eine Erneuerung des filmischen Realismus in Italien, als ricerca dell authenticita. Hierbei gewährleistete die Darstellung der Originalschauplätze eine höhere Wiedererkennbarkeit der Topografie beim Besuchen der Orte durch Touristen. Dieser von den Filmemachern also ursprünglich gar nicht bewusst eingesetzte Authentizitätseffekt erwies sich als erfolgreich, da hierdurch das nach Hattendorf wichtige Kriterium „authentisierender Überprüfbarkeit von Seiten der Rezipienten“ (Hattendorf 1999: 233) gewährleistet werden konnte.

Eine zentrale These dieses Kapitels ist, wie in der Einleitung angeführt, dass filmgeografische Authentizitätsdiskurse auch bei Filmen der dritten und vierten Simulationsstufe eine wichtige Rolle spielen: Wie in der Analyse zu belegen sein wird, macht die grundsätzliche geografische Übereinstimmung von filmischem Handlungsort und location einen wichtigen Teil der Authentizitätserwartung eines signifikanten Teils der Zuschauer aus. Filmgeografische Kongruenz ist vor allem deshalb für Zuschauer wichtig, weil sie zum einen auf kognitiver Ebene Filme als geografische Informationsquellen betrachten, die ihre Vorstellung von dem dargestellten Land deutlich beeinflusst und im Falle eines (film-)touristischen Besuchs ihnen bei der Antizipierung der „Atmosphäre“ des Urlaubslandes hilft. Zum anderen spielt diese Form von Kongruenz auch auf emotionaler Ebene eine bedeutende Rolle: Durch den Einsatz einer realen location wird der Handlungsort, der in seiner spezifischen Form nur auf der virtuellen filmischen Ebene existiert, mit dem lebensweltlichen Raum des Zuschauers verknüpft, sodass dieser einen Bezug zwischen Fiktion und Realität herstellen kann. Hierdurch erscheint ihm der Film bedeutsam für seine eigene Lebenswelt. Mit anderen Worten ragt die filmische Fiktion nicht nur durch einen realitätsbasierten Handlungsort, sondern auch durch den Einsatz einer realen location in die reale Welt des Zuschauers hinein.

Geografische Kongruenz ist aber bei Filmen der dritten Simulationsstufe gerade nicht gegeben, da hier Handlungsort und location geografisch auseinanderdriften, wodurch die potenzielle Gefahr besteht, dass die Geografie des Films und womöglich der gesamte Filmtext von Zuschauern als inauthentisch eingestuft wird und dadurch auch an emotionaler Bedeutsamkeit einbüßt. ${ }^{35}$ Der

\footnotetext{
${ }^{35}$ Während die filmgeografische (In-)Kongruenz objektiv feststellbar ist, ist filmgeografische (In-)Authentizität rezeptionsabhän-
} 
Handlungsort gibt in solchen Filmen aus Zuschauersicht vor, in geografischer Hinsicht etwas zu sein, das er nicht „ist“. Die neuseeländische location ist hierbei das primäre „de-authentisierende“ Element, da sie zwar real ist, aber bezüglich des Handlungsortes eine „falsche“ geografische Realität abbildet. Dieser Umstand kann als „Vertragsbruch“ gewertet und beispielsweise mit irritierten oder enttäuschten Rezensionen sanktioniert werden. Im Falle einer Sanktionierung durch den Zuschauer ist die Authentisierungsstrategie der Filmemacher missglückt. Hierbei wird der Filmtext in filmgeografischer Hinsicht als inauthentisch und damit sowohl hinsichtlich seiner Brauchbarkeit als geografische Informationsquelle als auch in emotionaler Hinsicht im schlimmsten Falle als unbefriedigend bzw. irreführend erachtet.

Hattendorf definiert Authentisierungsstrategien als „filminterne pragmatische Markierungen [...], die den Rezipienten in einem Spektrum impliziter oder expliziter Appelle dazu auffordern, einen, Wahrnehmungsvertrag' mit dem jeweiligen Film zu schließen“ (1999: 311). Welche Authentisierungsstrategien setzen Filmemacher nun bei Filmen der dritten und vierten Simulationsstufe konkret ein, um die Authentizitätserwartungen der Zuschauer nicht zu enttäuschen und sie stattdessen zu einem „Wahrnehmungsvertrag“ zu bewegen? Es gibt meines Ermessens insbesondere zwei Strategien, die hier relevant sind: I. die filmgeografische Delokalisierung auf der Filmtextebene und 2. die Vermarktung des Films auf paratextueller Ebene.

Die grundsätzliche Problematik bei Filmen der dritten/vierten Simulationsstufe ist, dass Handlungsort und location geografisch nicht übereinstimmen. Die Entscheidung, überhaupt an locations zu drehen, die sich geografisch vom Handlungsort unterscheiden, beruht meist entweder auf ökonomischen Erwägungen und/oder stellt eine Verlegenheitslösung dar: Begünstigt durch den allgemeinen Globalisierungsprozess konnte sich Neuseeland als location etablieren, die zum einen preisgünstig ist und zum anderen die notwendige politische Stabilität und vergleichsweise wenig bürokratische Hürden aufweist, um einen reibungslosen Produktionsablauf zu gewährleisten. Zwar hat das neuseeländische Department of Conservation (DOC) bestimmte Umweltschutzauflagen aufgestellt, die bei einem Filmdreh erfüllt werden müssen, ${ }^{36}$ explizite Drehverbote sind jedoch im Vergleich zu anderen Ländern selten. Diese Vorteile erklären die zunehmende Anzahl sogenannter runaway productions in Neuseeland, d. h. Filmen, die für den US-Markt bestimmt sind, aber in einem anderen Land gefilmt wurden.

\footnotetext{
gig, vom Zuschauer konstruiert.

${ }^{36}$ Beispielsweise musste beim Dreh von Lord of the Rings dem neuseeländischen Department of Conservation zugesichert werden, dass die empfindliche Vegetation vor Schäden geschützt wird (Roesch 2009: 42).
} 
Damit die neuseeländische location in Filmen der dritten/vierten Simulationsstufe ein anderes Land simulieren kann, muss die location delokalisiert werden. Der Begriff der „filmgeografischen Delokalisierung“ ist an Jutels Begriff des radical dépaysement angelehnt. Jutel schreibt hierzu: „Literally an ,out-of-nation-ness', dépaysement represents the simultaneous attraction of geographic defamiliarisation, and the separation of the lost homeland. In the case of New Zealand, the dépaysement that is left undefined is captured in the landscape“ (2004: 60). In der Regel wird die neuseeländische location so ausgewählt, dass sie dem vom Handlungsort darzustellenden Land geografisch zumindest in den Grundzügen ähnelt, um den Aufwand einer geografischen „Retusche“ möglichst gering zu halten. Hierbei machen sich die Filmemacher zunutze, dass bei der globalen Rezeption des Films ein großer Teil der Zuschauer weder mit den geografischen Details ${ }^{37}$ der location Neuseeland, noch mit denen des durch den Handlungsort darzustellenden Landes vertraut ist. Allerdings ist die geografische Ähnlichkeit zumeist nicht groß genug, um nicht doch eine Wahrnehmung des Films als geografisch inauthentisch zu riskieren. Deshalb weist Leotta auf den wichtigen Punkt hin, dass zum dépaysement bzw. zur Delokalisierung das Entfernen möglichst vieler neuseelandspezifischer Charakteristika bzw. „Marker“ der location und anschließend das zielgerichtete „Befüllen“ mit möglichst bekannten „Markern“ des durch den Handlungsort darzustellenden Landes gehört (20II: 137). Ziel dieser Authentisierungsstrategie ist es, bei dem Zuschauer den Eindruck zu erwecken, dass tatsächlich dort gedreht wurde, wo der Handlungsort vorgibt zu sein (also nicht in Neuseeland). Wenn diese Strategie glückt, akzeptiert der Zuschauer die Authentizitätssignale und empfindet den Handlungsort als geografisch glaubwürdig.

MacCannell zufolge verhält sich der von ihm geprägte Begriff des „Markers“ zu einer Touristenattraktion analog zu der Beziehung zwischen Signifikant und Referent, d. h. der Marker repräsentiert die Attraktion, wobei die Touristenattraktion sich semiotisch aus der Beziehung zwischen Marker und Attraktion zusammensetzt. Des Weiteren kann zwischen on sight-Markern, die sich an der realen Attraktion befinden und off sight-Markern, die von einem beliebigen Ort aus auf die Attraktion verweisen, differenziert werden, wobei es sich in Filmen immer um off sight-Marker handelt (I999: IIO-III). Der Einsatz von Markern ist eine wichtige Strategie der Filmemacher, um Authentizitätseffekte bei der Darstellung des Handlungsortes auszulösen.

\footnotetext{
${ }^{37}$ Mit „geografischen Details“ sind hier charakteristische Eigenarten einer bestimmten Geografie gemeint, die sie einzigartig und von anderen Geografien unterscheidbar machen, beispielsweise endemischer Flora und Fauna sowie charakteristischer Geormorphologie. So weist beispielweise der neuseeländische Milford Sound eine charakteristische Geomorphologie auf und hat für Neuseeland einen geradezu nationalsymbolischen Charakter. Aber auch stereotypische Bauten und Gebäude als Teil der Kulturlandschaft, beispielsweise „typisch japanische“ Tempel können den Handlungsort als ,japanisch“ markieren.
} 
An dieser Stelle empfiehlt sich die Differenzierung zwischen „nationalspezifischen“ und „nationaltypischen“ Markern: Während die als Wildnis wahrgenommenen Gebiete Neuseelands sich ausschließlich auf Neuseeland beziehen und dessen Einzigartigkeit bezeugen, sind Marker wie beispielsweise die ebenfalls für Neuseeland als typisch erachteten und in der Tourismuswerbung ebenfalls häufig dargestellten pastoralen Weideflächen mitsamt ihren Schafen und Kühen mehrdeutig, da grüne, von sanften Hügeln geprägte Weideflächen auch typisch für viele andere Länder sind (z. B. England, Schottland, Irland). Urry sieht Touristen als „Quasi-Semiotiker“ an, die das besuchte Land nach nationalstereotypischen Zeichen absuchen, die ihre vorher durch Medienexposition gebildeten Erwartungen an das Land bestätigen (1990: I2). So suchen beispielsweise Touristen in Deutschland den typischen Biergarten, in Frankreich das typische Chateau und in England das rurale „Olde England.“ Diese Stereotypen entsprechen dem, was Barthes in seiner beispielhaften Analyse einer Panzani-Werbung als „Italianicity“ bzw. „Italienischkeit“ bezeichnet. Hierbei stellt er klar, dass „Italienischkeit“ nicht mit Italien gleichzusetzen ist, sondern vielmehr die kondensierte Essenz von allem darstellt, das als „typisch italienisch“ gilt (1977: 48).

Aber auch wenn allzu neuseelandspezifische Marker entfernt werden, kann die Simulation als „Täuschung“ entlarvt werden, entweder weil Zuschauer die Inkongruenz des Handlungsortes immer noch erkennen oder weil sie durch Para- oder Metatexte von der tatsächlichen location erfahren. Dies ist, wie später in der Analyse zu erläutern sein wird, beispielsweise bei The Waterhorse: Legend of the Deep der Fall, wo Loch Ness durch einen neuseeländischen See simuliert wird und einige Rezensenten sich irritiert darüber zeigen. Hier haben die Metatexte, die über die tatsächliche location Neuseeland informieren, genau den gegenteiligen Effekt wie der Marker bei MacCannells „Mondstein“-Beispiel: Während für die Museumsbesucher ein absolut unauffälliger Stein durch die Plakette mit der Aufschrift „Moonstone“ eine besondere Bedeutung als „authentischer“ Mondstein erfährt (1999: II3), markiert der Metatext „filmed in New Zealand“ den Handlungsort „Schottland“ als geografisch „inauthentisch“. Dies belegt, dass Landschaften im Film in den Augen der Zuschauer - trotz des relativ sorglosen Umgangs der Filmemacher - nicht beliebig austauschbar sind: Die Vermarktung Neuseelands als „semiotisch flexibles“ landschaftliches „Chamäleon“, mit dem problemlos alle möglichen ausländischen Handlungsorte simuliert werden können, stößt also mitunter an ihre Grenzen.

Gerade bei der Analyse der Markierung der Filmlandschaft bietet sich die Einbeziehung von Zuschauerrezensionen im Rahmen einer Viewer Response Theory an, da Zuschauer in ihren 
Rezensionen erstaunlich oft ein Interesse an der tatsächlichen location eines Films zeigen, was ein klares Indiz für das filmtouristische Potenzial landschaftsfokussierter Filme darstellt. Die Viewer Response Theory stellt eine Modifikation der Reader Response Theory dar, die statt Literatur den Spielfilm als Analysegegenstand hat und den theoretischen Gegenpol zur Auteur-Theorie darstellt. Bei der Viewer Response Theory wird davon ausgegangen, dass der Filmtext nicht eine singuläre objektive Bedeutung hat, sondern dass es immer mehrere Lesarten eines Filmtextes gibt und dass eine bestimmte Bedeutung des Filmtextes erst durch die Interaktion zwischen Zuschauer und Filmtext entsteht (Piturro 2008: 7).

Zur besseren Veranschaulichung soll nun der Prozess der filmgeografischen Delokalisierung noch einmal grafisch dargestellt werden:

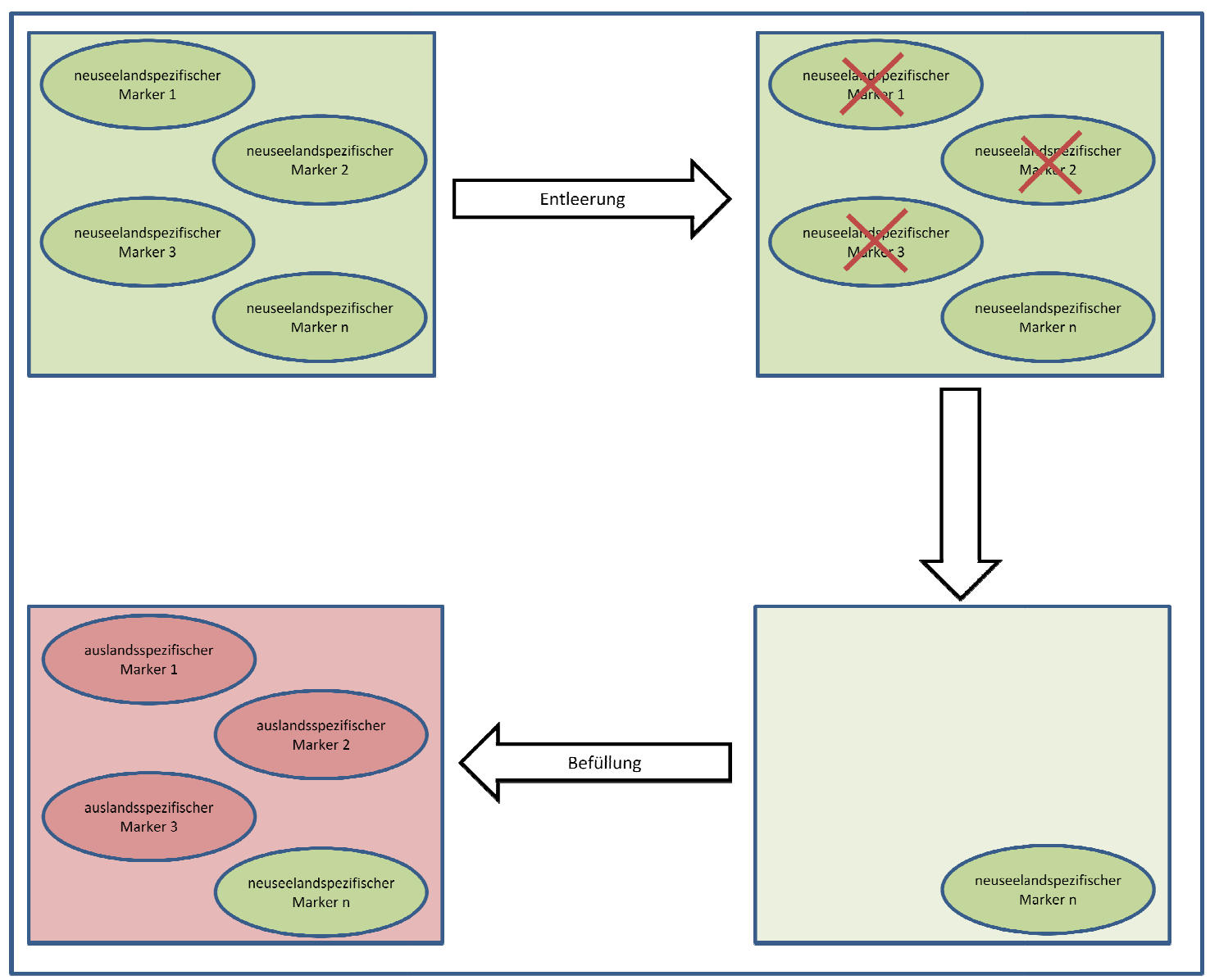

Abbildung 3.2: Delokalisierung und Entleerung von/Befüllung mit Landschaftsmarkern

Die Abbildung illustriert den semiotischen Prozess des Entleerens und Befüllens, in dessen Verlauf die neuseeländische location in einen nicht-neuseeländischen Handlungsort verwandelt wird. Während des Filmdrehs und/oder in der Postproduction werden so viele neuseelandspezifische Land- 
schaftsmarker wie möglich aus der location entfernt (Entleerung), da diese nur bei der Simulation eines ausländischen Handlungsortes stören würden. Die Abbildung verdeutlicht zudem, dass meines Ermessens diese Entleerung nie vollständig sein kann, da eine Entfernung sämtlicher neuseelandspezifischer Landschaftsmarker der Entfernung der neuseeländischen location an sich nahezu gleichkommen würde und zudem zu zeit- und kostenaufwendig für die Filmemacher wäre. Stattdessen muss sich auf diejenigen Marker konzentriert werden, die als zu markant erachtet werden: So würde die Darstellung des bekannten Milford Sound aufgrund seiner hohen Bekanntheit bei der Simulation eines ausländischen Handlungsortes die geografische „Authentizität“ des Films prinzipiell wesentlich stärker beeinträchtigen als die Darstellung einer weniger bekannten neuseeländischen Region, die leichter für einen ausländischen Landschaftsmarker gehalten bzw. damit „verwechselt“ werden kann. Anschließend wird die entleerte Landschaft mit auslandsspezifischen Landschaftsmarkern befüllt, beispielsweise bei Last Samurai durch die digitale Platzierung des als typisch japanisch erachteten Mt. Fuji in der neuseeländischen location, oder US-Flaggen in Without a Paddle, um eine neuseeländische Kleinstadt in eine amerikanische Kleinstadt zu verwandeln. Hierdurch soll die zugrundeliegende neuseeländische location verschleiert bzw. maskiert werden. Durch die zwangsläufig unvollständige Entleerung der location kann es je nach Zuschauertyp zu einem kognitiven und emotionalen Konflikt aufgrund der Pluralisierung der Zeichenbedeutungen kommen. Folgende Grafik zeigt in Anlehnung an Ogden und Richards dreiteiliges semiotisches Modell (1923: 30) die unterschiedlichen semiotischen Konstellationen bei Filmen der ersten und dritten bzw. vierten Simulationsstufe: 


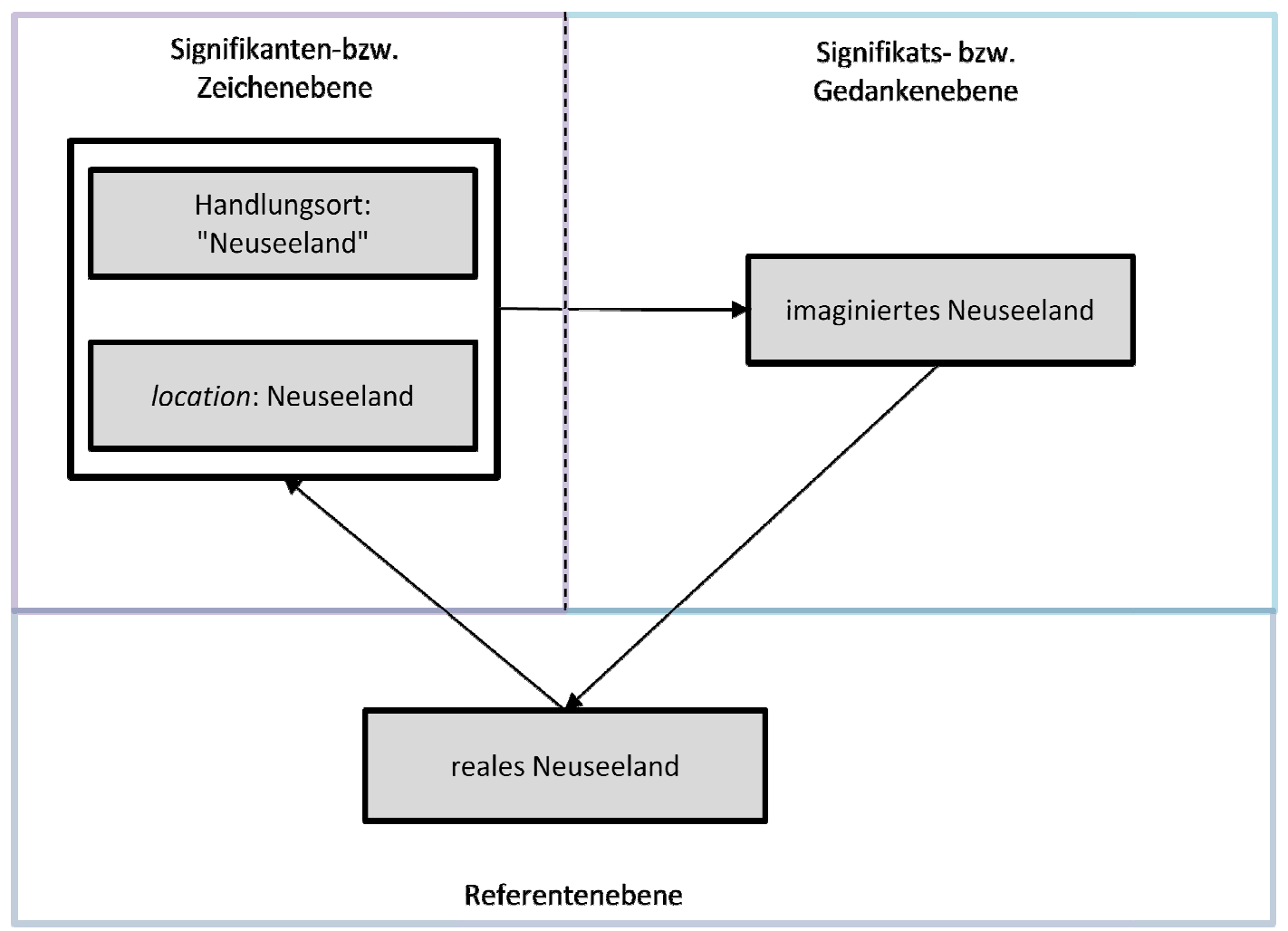

Abbildung 3.3: I. Simulationsstufe

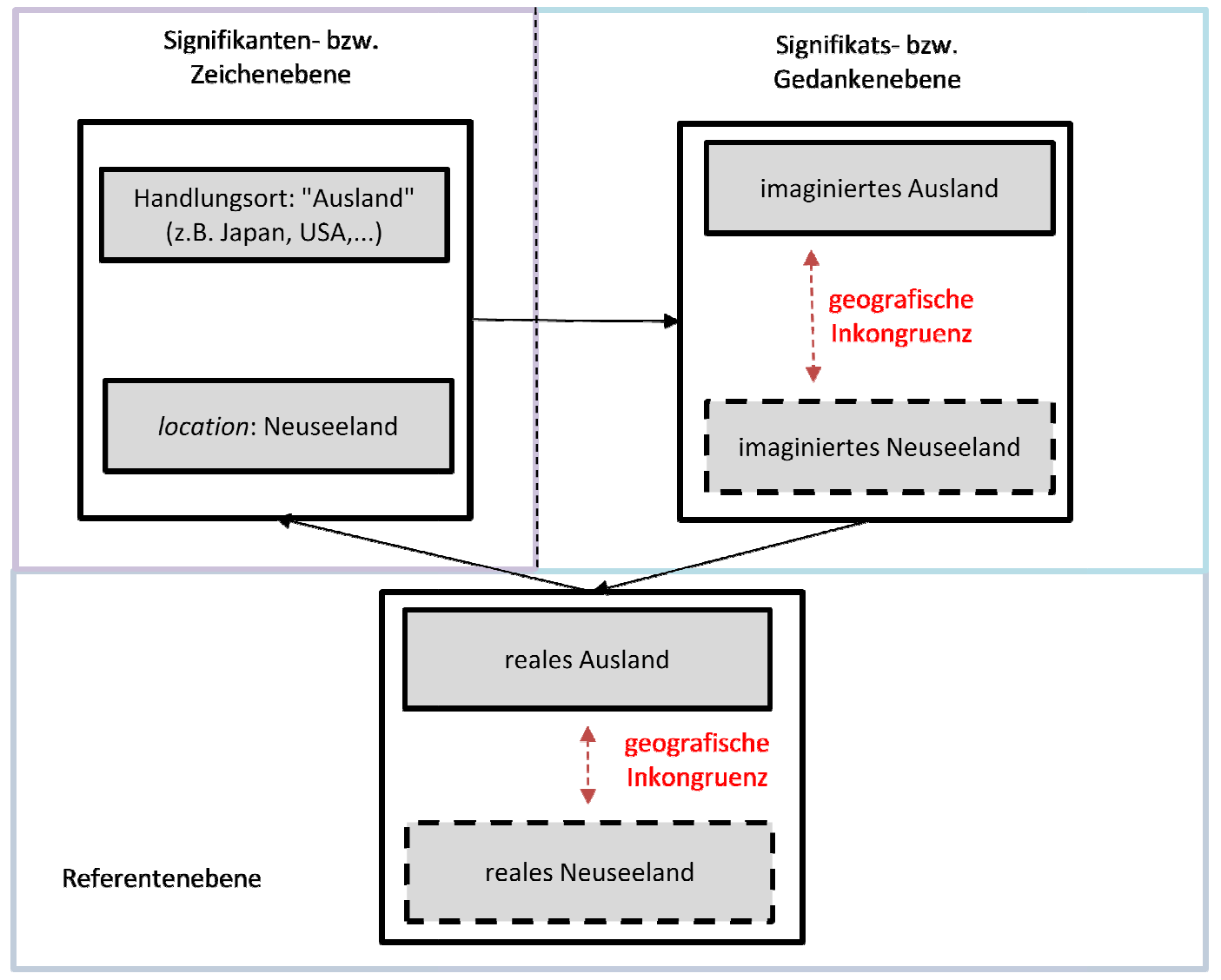

Abbildung 3.4: III. / IV. Simulationsstufe 
Die Abbildungen zeigen, dass ein Film der ersten Simulationsstufe zwei Signifikanten hat, die beide auf der Signifikatsebene die Vorstellung von Neuseeland hervorrufen. Durch den Einsatz einer neuseeländischen location wird dem Zuschauer zugleich signalisiert, dass der Film eine reale geografische Ebene hat, weil die im Film gezeigte location einen Ausschnitt aus der realen Geografie Neuseelands, dem Referenten, darstellt. Die location ist somit zugleich Teil des virtuellen, filmischen Handlungsortes als auch eine Untermenge der (physisch existenten) Geografie Neuseelands. Der Film wird dadurch in der Regel als filmgeografisch authentisch wahrgenommen, da der Zuschauer weiß, dass es sich bei Neuseeland um einen realen, lebensweltlichen Raum handelt. Ein Film der dritten/vierten Simulationsstufe hingegen hat ebenfalls zwei Signifikanten (Handlungsort und location), die auf geografisch unterschiedliche Signifikate verweisen. Wenn der Zuschauer die genaue location nicht kennt, kann ihm der Film wie ein Film der ersten Simulationsstufe erscheinen (s. o.). Wenn der Zuschauer jedoch Kenntnis von der location hat (rote/gestrichelte Linien), kann er die Filmlandschaft simultan mit dem vom Handlungsort dargestellten Land und Neuseeland assoziieren (Zeichenpluralisierung). Dieser semiotische Konflikt kann dazu führen, dass der Film als filmgeografisch inauthentisch wahrgenommen wird, da der erwartete Bezug zum realen Ausland fehlt und stattdessen nur das reale Neuseeland als „falscher“ Referent dient, der mit dem Handlungsort in geografischer Hinsicht nicht in Übereinstimmung zu bringen ist. Zugleich eröffnet dieser Bezug zu Neuseeland aber für den Filmtouristen die Möglichkeit, entweder die location oder aber das vom Handlungsort repräsentierte Land zu besuchen. ${ }^{38}$

Bei einigen der zu analysierenden Filme wurden gleich locations mehrerer Länder eingesetzt. So wurden für The Last Samurai, dessen Handlungsort das Japan des 19. Jahrhunderts ist, neuseeländische, aber auch japanische locations eingesetzt. Hierdurch wird die Situation auf semiotischer Ebene besonders komplex: Da sich nicht auf eine location beschränkt wurde, kann der Zuschauer sich letztlich nicht mehr sicher sein, ob eine bestimmte Szene gerade in Neuseeland oder aber Japan gedreht wurde. Letztlich handelt es sich bei The Last Samurai folglich um ein Hybrid zwischen zweiter und dritter Simulationsstufe, da der Handlungsort „historisches Japan“ stellenweise auch tatsächlich durch eine japanische location simuliert wird. Hierdurch werden die Zeichenbedeutungen nicht nur pluralisiert, es kommt auch zu einer Zeichenindeterminiertheit, d. h. die Zeichen

\footnotetext{
${ }^{38}$ Beispielweise weist Beeton darauf hin, dass Filmtouristen nach Mel Gibsons Braveheart sowohl Schottland (Handlungsort) als auch Irland (location) besucht haben (2005: 58-60).
} 
können nicht mehr eindeutig zugeordnet werden. Folglich kann sich der Zuschauer auch nicht mehr sicher sein, dass er eine für ihn emotional bedeutsame Landschaft, in die er seine Sehnsüchte investiert (beispielsweise eine pastorale Idylle) auch mit dem „richtigen“ realen Raum in Bezug setzt, was in der Folge Verwirrung und emotionale Distanzierung erzeugen kann.

\subsubsection{V. + VII. Simulationsstufe: Archaisch-mythische Ästhetik, (Hyper-)Sublimität, Foto- realismus als Authentisierungsstrategien und Entlokalisierungsprozess}

Auch in (zwangsläufig effektlastigen) Fantasyfilmen, die rein fiktive Handlungsorte und virtuelle Geografien aufweisen, wird Wert auf Authentizität gelegt, allerdings beziehen sich Fantasywelten nicht unmittelbar auf reale Räume und werden daher auch nicht direkt an ihnen gemessen. Woran wird nun in solchen Filmen die Authentizität ihrer fiktiven Geografien bemessen? Die Glaubhaftigkeit einer Fantasywelt bestimmt sich meiner Ansicht nach an verschiedenen Parametern, die sich auf komplexe Weise gegenseitig beeinflussen: Den seit dem Aufkommen von Fantasyliteratur und -filmen etablierten Darstellungskonventionen von Fantasywelten (Intertextualität), den persönlichen kreativen Visionen der Filmemacher und den durch das Lesen von Fantasyliteratur (einschließlich der darin vorkommenden illustrativen Zeichnungen) und Betrachten von Fantasyfilmen evozierten Vorstellungen in den Köpfen der Leser/Zuschauer.

Fantasywelten weisen meines Ermessens generell zwei Konstanten auf, die mittlerweile als konstitutiv für deren Glaubwürdigkeit betrachtet werden können: Archaik und Sublimität. Der erste Aspekt, die archaisch-mythische Atmosphäre, bedient hierbei in seiner, feudale Strukturen idealisierenden Darstellung, gezielt die nostalgische Sehnsucht der Leser/Zuschauer nach einem Goldenen Zeitalter mit einfachen sozialen Ordnungen, klaren moralischen Werten und einer quasi binären Kontrastierung von Gut und Böse. Hierbei versinnbildlicht die „Kulturlandschaft“ mit ihren einfachen agrarischen Siedlungen, Burgen, Schlössern, Festungen und Ruinen diese Ideale und verleiht der fiktiven Welt (pseudo-)historische Tiefe.

Fantasywelten sind zudem von stereotypischen Landschaftästhetiken geprägt, d. h. pastoraler, sublimer und gotbic-Ästhetiken, wobei insbesondere die Sublimität den „fantastischen“ Charakter dieser Welten ausmacht. Das Sublime bzw. das Erhabene wurde von den Philosophen Edmund Burke und Immanuel Kant thematisiert und als ein ästhetisches Phänomen betrachtet, das die empfundene Ohnmacht des Menschen angesichts übermächtig und unendlich erscheinender Naturgewalt zum Ausdruck bringt und sowohl Gefühle der Angst als auch der Euphorie hervorru- 
fen kann. Der Begriff des Sublimen ist auch auf menschliche Architektur ausgeweitet worden (technological sublime [Nye, 1994]), was für die späteren Filmanalysen ebenfalls relevant sein wird, da in Fantasywelten nicht nur die Natur, sondern auch die Schlösser und Festungen oft überdimensional und gewaltig erscheinen. Da die Fantastik von Fantasywelten oftmals natürliche physikalische Gesetzmäßigkeiten überschreitet, möchte ich diese Art von Ästhetik als „Hypersublimität“ bezeichnen, d. h. eine Sublimität, die gewissermaßen „sublimer als sublim“ ist.

Diese (hyper-)sublime Ästhetik wird in modernen Fantasyfilmen besonders betont und dabei bewusst eine sensorische Überwältigung des Zuschauers angestrebt. Hoberg spricht in diesem Zusammenhang unter Rekurs auf Wolfgang Welschs Konzept der „Anästhetisierung“ von einer durch wiederholte sensorische Überreizung erfolgenden Abstumpfung der Sinne des Zuschauers, was wiederum eine Reizsteigerung vonnöten macht (1999: 213, 214). Um die Gunst des Zuschauers zu gewinnen, treten Filmemacher in einen Wettbewerb und versuchen sich in puncto Fantastik gegenseitig zu übertrumpfen, was Hoberg als „Zwang zur Gigantomanie“ bezeichnet (1999: 9, I42, 218). Um diese ständige Reizsteigerung in modernen Fantasyfilmen zu ermöglichen, die immer mehr dem entsprechen, was Tom Gunning als moderne Form des visuellen Spektakels, dem cinema of attractions, bezeichnet (1986), ist der Einsatz von CGI, das nahezu unbegrenzte Darstellungsmöglichkeiten verspricht, bereits zu einer unumgänglichen Notwendigkeit geworden. Zwar ist die Darstellung hypersublimer Welten bereits früher prinzipiell möglich gewesen, beispielsweise durch den Einsatz von matte paintings, ${ }^{39}$ aber die immersiven, vertiginösen Kamerafahrten, die die Sublimität in ihrer Dreidimensionalität voll zur Geltung bringen und für die Peter Jacksons Filme sowie James Camerons Avatar bekannt sind, können nur mit digitaler Technologie verwirklicht werden.

Hier kommt allerdings neben den generellen Aspekten der Archaik und der Sublimität, die auch auf Fantasyliteratur zutrifft, der filmspezifische Aspekt des Fotorealismus als drittes wichtiges Authentizitätskriterium ins Spiel, das umso bedeutsamer wird, desto mehr CGI eingesetzt wird. Warum aber erwarten Zuschauer moderner Fantasyfilme überhaupt Fotorealismus? In der Beantwortung dieser Frage erweist sich Ecos Argument als hilfreich, dass fiktive Welten „Parasiten der wirklichen Welt" seien und dass zum Verständnis selbst fantastischster Welten Zuschauer von ihrer eigenen Welterfahrung ausgingen (1994: II2-II3). Somit müssen auch fiktive Welten Elemente des

\footnotetext{
${ }^{39}$ Bei matte paintings handelt es sich um fotorealistisch gemalte Hintergründe, vor denen die Schauspieler agieren. Diese Technik ist dem Theater entlehnt, hat in Filmen aber den Nachteil, dass die Perspektive starr festgelegt ist und keine dynamischen Kamerafahrten zulässt, da dem Zuschauer sonst sofort auffallen würde, dass es sich nicht um eine location, sondern „nur“ eine gemalte Landschaft handelt.
} 
Realen enthalten, um zugänglich zu bleiben. Durch den Einsatz realer locations in Filmen der fünften Simulationsstufe wird ein Realitätseffekt erzielt, was ebenfalls eine Art „parasitärer“ Entlehnung aus realen Geografien darstellt, um der Fantasywelt dieses Element realer Tiefe zu verleihen. Wenn nun digitale Landschaften mit locations kombiniert werden oder erstere letztere ersetzen, streben Filmemacher den gleichen Realismuseffekt an, indem sie die digitalen Elemente fotorealistisch und damit möglichst ununterscheidbar von den ohnehin immer fotorealistischen locations konstruieren. Auch bei den digital generierten Landschaften von Filmen der siebten Simulationsstufe dient der Fotorealismus als ein Surrogat für die fehlenden realen locations, die bisher eine lebensweltliche Rückbindung für den Zuschauer ermöglichten. Durch den Fotorealismus soll die Digitalität der digitalen Effekte verschleiert werden, sie sollen „natürlich“ erscheinen.

Was also einen wichtigen Reiz für Zuschauer von Fantasyfilmen darstellt, ist, dass trotz aller physikalisch unmöglichen Hypersublimität das Geschehen dennoch „real“ und immersiv wirkt, was North folgendermaßen ausdrückt: „When we see, onscreen, an impossible event happening in ,photorealistic ' CGI, we are invited into the fantasy that it has actually been recorded photographically, and thus that it has taken place in the real world“ (2008: 12). Dies gilt allerdings nur, wenn für den Zuschauer die Digitalität der digitalen Effekte weitgehend unsichtbar bleibt. Wenn die zur Simulation dieser Fantasywelten eingesetzten digitalen Effekte jedoch von den Zuschauern als „schlecht“ eingestuft werden, d. h. dass sie dem Zuschauer unmittelbar als künstliche Effekte ins Auge springen, wird diese virtuelle Welt als inauthentisch eingestuft. Hierbei wird die unbefriedigende Darstellung der Fantasywelt in abwertender Weise z. B. mit der plakativen, nichtnaturalistischen Darstellungsweise klassischer Zeichentrickfilme oder mit „Computerspielgrafik“ verglichen. ${ }^{40}$

Dasselbe gilt für die digitale Generierung historischer Artefakte, um dem Handlungsort historische Tiefe zu verleihen: Ein digital generiertes, aber total „verpixeltes“ Fantasyschloss beispielsweise würde nicht nur den Realismuseffekt zerstören, sondern zugleich auch auf seinen Status als digitales, also hochmodernes Artefakt verweisen, was dem Bestreben der Filmemacher nach der

\footnotetext{
${ }^{40} \mathrm{Mit}$,klassischem Zeichentrickfilm“ sind hier noch von Hand gezeichnete und anschließend animierte Filme gemeint wie es vor allem die Disneyfilme vor der Ära moderner 3D-Animationsfilme wie Toy Story (1995) waren. Klassische Zeichentrickfilme zeichnen sich ungeachtet ihres durchaus hohen künstlerischen Wertes durch eine zweidimensionale Plakativität (Richter 2008: 73) aus, die sich vor allem in der Darstellung der Landschaft bemerkbar macht, der die perspektivische „Tiefe“ fehlt, was aber durchaus auch ihren Charme und Märchencharakter ausmacht. In der Entwicklung von Computerspielen wird zwar wie bei Fantasy/Science Fiction-Filmen nach Fotorealismus gestrebt, jedoch hinkt diese Entwicklung dem Grad des Fotorealismus von Hollywoodfilmen immer einen Schritt hinterher, da heimische PCs stets leistungsschwächer sind als die Hochleistungsrechner von Postproduction-Anlagen wie beispielsweise WETA digital. Sowohl von der Plakativität von Zeichentrickfilmen als auch von der „schlechten“ Grafik von Computerspielen wollen sich Filmemacher von Fantasyfilmen in jedem Fall distanzieren.
} 
Simulation historischer Tiefe bzw. oben erwähnter Archaik geradezu diametral entgegenstehen würde. ${ }^{41}$ Weil der neuseeländischen location aber die historische Tiefe anderer Länder wie England oder Schottland fehlt, müssen Filmemacher zur „Historisierung“ Neuseelands zwangsläufig entweder auf Filmsets oder digitale Effekte zurückgreifen.

Die heutige Möglichkeit, locations sukzessive durch digitale Landschaften zu ergänzen oder auch zu ersetzen, lässt sich semiotisch folgendermaßen darstellen:

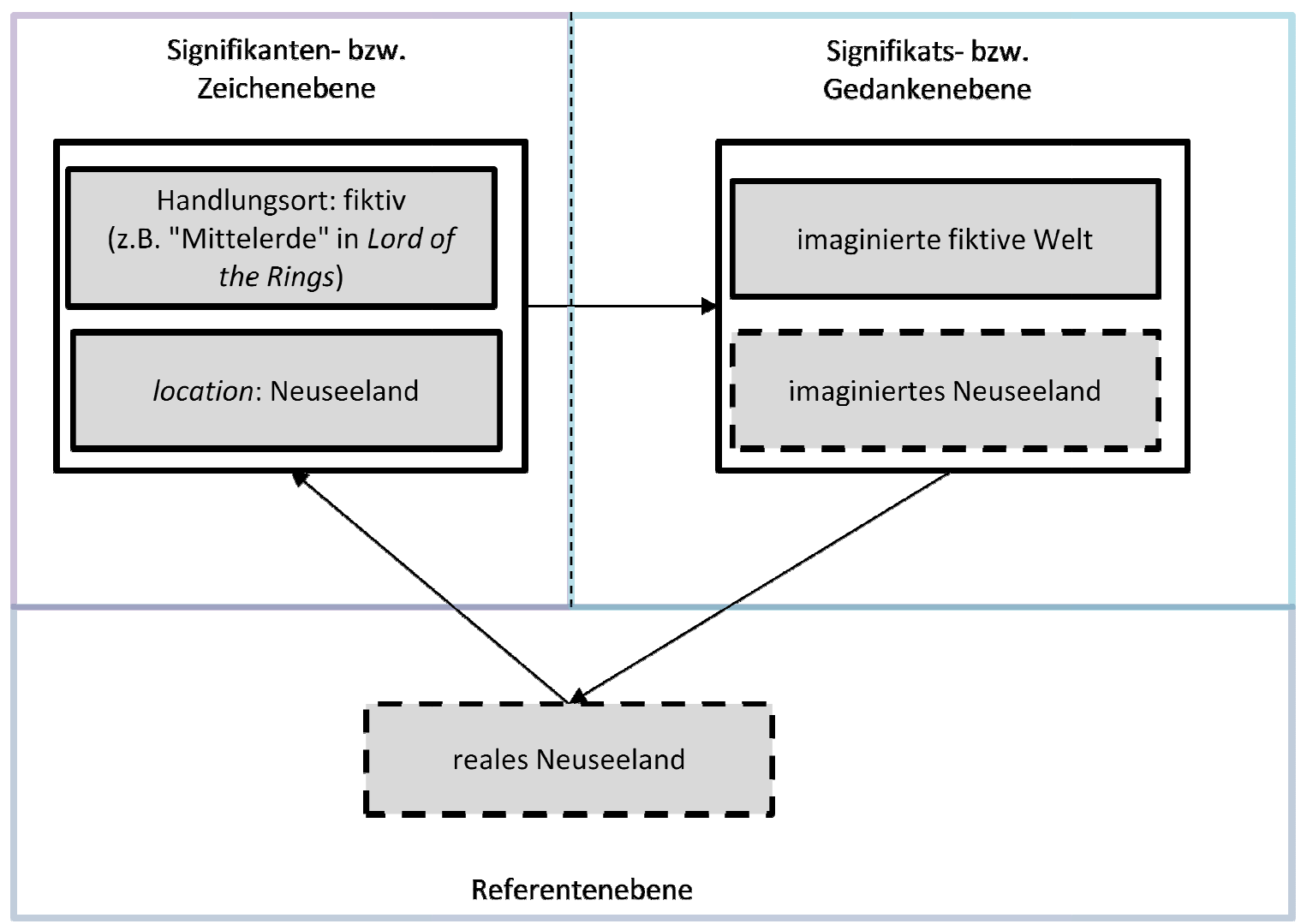

Abbildung 3.5: V. Simulationsstufe

\footnotetext{
${ }^{41}$ Ähnlich argumentiert North, wenn er schreibt: „The production of a special effect denotes the use of a technique specifically designed to alter, enhance or create an element of the diegesis. [...] If the discrepancies between the real and its simulation are too jarringly pronounced, we dismiss them as , bad' special effects, those which have reminded us too forcibly that Superman is not really flying or that the Titanic is just a glorified bath toy bobbing in a pond on the studio backlot“ (2008: 4).
} 


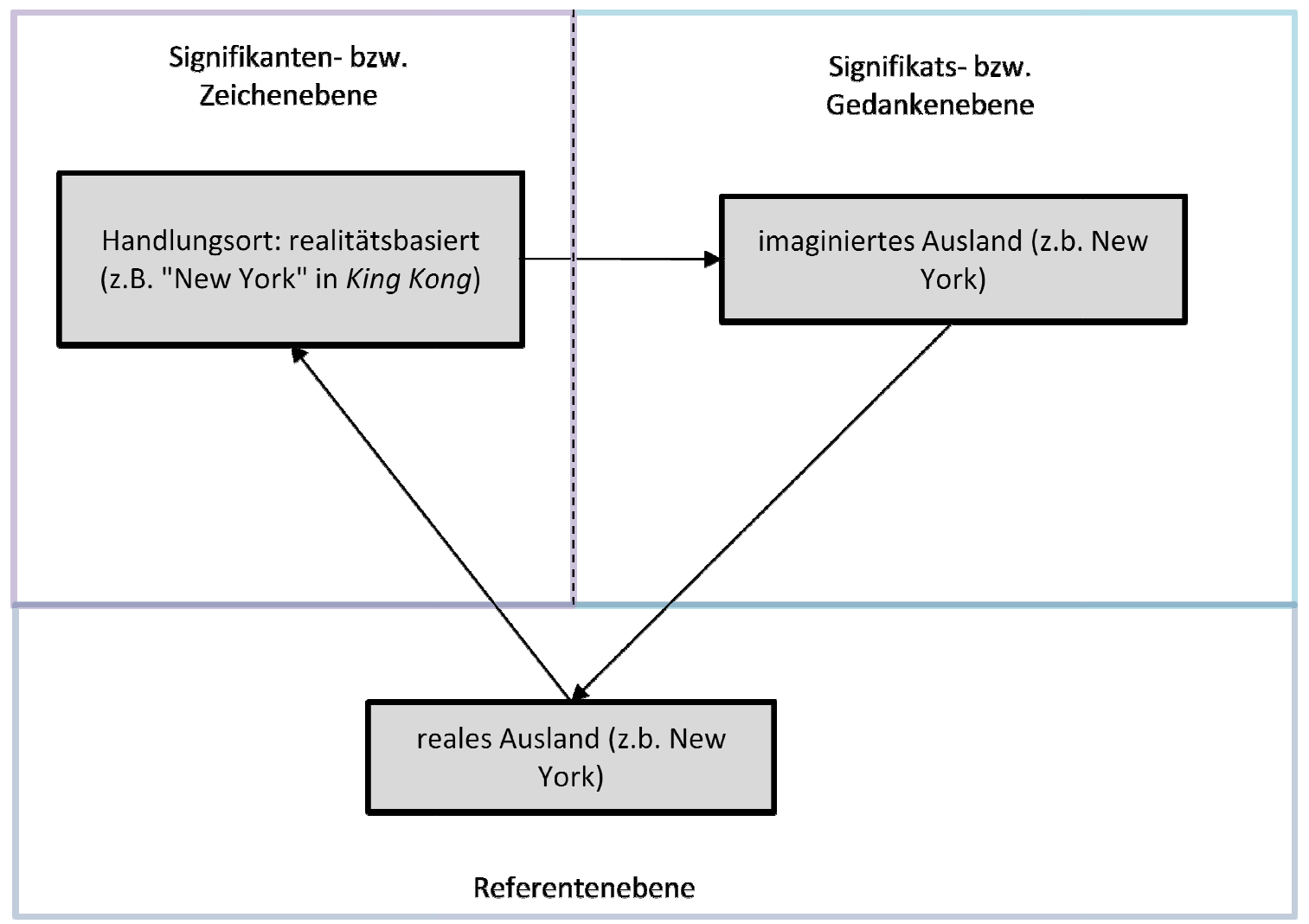

Abbildung 3.6: VI. Simulationsstufe mit realitätsbasiertem Handlungsort

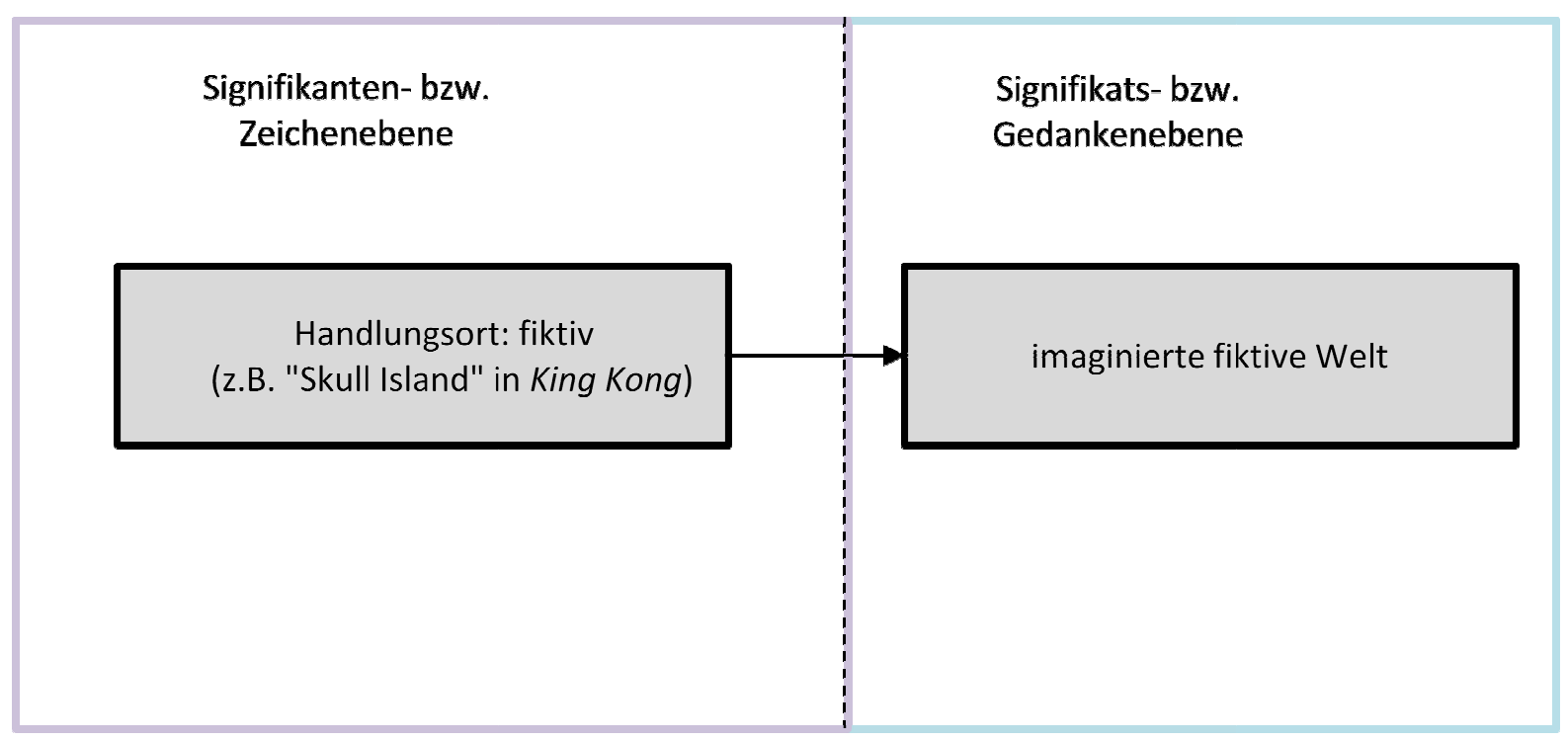

Abbildung 3.7: VII. Simulationsstufe mit fiktivem Handlungsort

Die Abbildungen zeigen, dass bei der fünften Simulationsstufe der fiktive Handlungsort semiotisch nur deswegen auf einen realen Ort, in diesem Fall Neuseeland, verweist, weil neuseeländische locations eingesetzt wurden - und dies auch nur unter der Voraussetzung, dass der Zuschau- 
er aufgrund von Para-/Metatexten weiß, dass reale locations eingesetzt wurden und daher den Handlungsort mit ihnen assoziieren kann. Bei der sechsten Simulationsstufe wird keine location mehr eingesetzt, wobei aber dennoch aufgrund des realitätsbasierten Handlungsortes wie beispielsweise dem digital generierten New York in King Kong ein referenzieller Bezug zum realen New York besteht, in dem Sinne, dass sich das digitale New York und das reale New York „ähneln“. Diese Ähnlichkeit kann mit dem peirceschen Begriff des Ikons (I876: 56) beschrieben werden, während ein indexikalischer, d. h. zwingend kausaler Bezug zwischen digitalem und realem New York fehlt ${ }^{42}$ - ganz im Gegensatz zu einem tatsächlichen Filmdreh in New York, bei dem eine semiotische Doppelreferenzialität vorliegt, nämlich sowohl ein indexikalischer als auch ikonischer Bezug zwischen Handlungsort und location.

Bei Filmen der siebten Simulationsstufe schließlich wird auf reale locations verzichtet, wodurch eine location oder ein vom Handlungsort repräsentiertes Land als Referent entfällt. Im Falle von „Skull Island“ in King Kong, kann der Zuschauer keine reale Geografie mit dem Handlungsort assoziieren und weder „Skull Island“ noch eine location besuchen. Allerdings soll an dieser Stelle bereits darauf hingewiesen werden, dass insbesondere bei Avatar zur Generierung der digitalen Landschaften fotografische Texturen eingesetzt wurden, die durch sogenanntes „Sampling“ chinesischer, hawaiianischer und neuseeländischer Regionen gewonnen wurden. Ob diese Fragmente realer Geografien für Zuschauer doch noch eine Referenzialität begründen, hängt von der Art und dem Umfang der fotografischen Samples ab und wird in der Analyse beantwortet werden.

\subsubsection{Rolle der Para- und Metatexte im Authentizitätsdiskurs}

Zu den Paratexten eines Films gehören insbesondere diejenigen Texte, die den Film vermarkten, d.h. Trailer, offizielle Webseiten-Auftritte, die Filmcredits, das DVD-Cover und Making Ofs. Während Trailer lediglich eine kurze Vorschau auf den Filminhalt bieten, können vor allem Filmcredits, offizielle Webseiten-Auftritte und Making Ofs auch Informationen zu den Produktionshintergründen des Films liefern. Hierbei wird auch oft auf die location hingewiesen. Während dies bei Filmen der fünften Simulationsstufe relativ unproblematisch ist, kann der location-Hinweis bei Filmen der dritten und vierten Simulationsstufe den Zuschauer auf die geografische Inkongruenz zwischen location und Handlungsort hinweisen, was dieser dann unter Umständen als „Inauthentizitätssignal“ werten kann. Obwohl Filmemacher in Making Ofs von Filmen der dritten

\footnotetext{
${ }^{42}$ Auf den Verlust der Indexikalität durch den Einsatz von CGI ist in der Forschungsliteratur mittlerweile vielfach hingewiesen worden, siehe z. B. Hoberg (1999), North (2008), Richter (2008), Prince (2012).
} 
und vierten Simulationsstufe vielfach auf andere Authentisierungsstrategien hinweisen, wie beispielsweise das Bemühen, die historische Landschaft des Handlungsortes an der location akkurat nachzubilden oder die „verblüffende“ Ähnlichkeit zwischen location und Handlungsort betonen, ist es fraglich, ob Zuschauer in jedem Fall diese Kompensationsbemühungen als Ausgleich zur filmgeografischen Inkongruenz akzeptieren oder ob für sie beispielsweise die historische Authentizität untrennbar mit filmgeografischer Authentizität zusammenhängt. Insbesondere in Making Ofs können neben der oben behandelten filmgeografischen Delokalisierung weitere Authentisierungsstrategien eingesetzt werden, unter anderen die der Projektion, der Kompensation und der Autorisierung.

Mit „Projektion“ ist hier gemeint, dass Filmemacher ihre eigene Sichtweise auf die Zuschauer übertragen, wenn sie beispielsweise ihre persönliche Ansicht, dass location und Handlungsort sich „verblüffend ähneln“würden, nicht als persönliche Ansicht, sondern als objektive Tatsache darstellen wollen und damit unterstellen, dass dieser Umstand für Zuschauer ebenso „offensichtlich“ und „unbestreitbar“ sein müsse. Die Strategie der Kompensation hingegen stellt den Versuch der Filmemacher dar, den Nachteil filmgeografischer Inkongruenz durch den Verweis auf andere „authentisierende“ Produktionsaspekte ausgleichen zu wollen. So können die Filmemacher im Making Of eingestehen, dass die neuseeländische location zwar nicht mit dem nichtneuseeländischen Handlungsort (z. B. Japan, USA etc.) übereinstimme, dass aber immerhin nicht komplett im Filmstudio gedreht worden sei, sondern on location. Mit dem Begriff der Autorisierung schließlich ist das Bemühen gemeint, durch Involvierung von Experten in die Filmproduktion den Film glaubwürdiger erscheinen zu lassen - beispielsweise durch das Mitwirken eines Extrembergsteigers in einem Bergfilm wie Vertical Limit.

Wie bei Filmen der dritten und vierten Simulationsstufe wird auch bei der Vermarktung von Filmen der fünften bis siebten Simulationsstufe darauf geachtet, das Filmprodukt als authentisch zu vermarkten. Während bei Lord of the Rings noch die Erwähnung des Drehs in Neuseeland eine wichtige Rolle spielte und der neuseeländischen Tourismusindustrie einen signifikanten $\mathrm{Zu}$ wachs bescherte, wurde auch hier in Making Ofs ein zweiter Schwerpunkt auf die digitale Erschaffung und Authentizität der Welt „Mittelerde“ selbst gelegt. Hierbei kann das Making Of trotz der Offenlegung filmischer „Tricks“ durch den Verweis auf die Originalität, Kreativität und Leidenschaft der Filmemacher und „Weltendesigner“ den Eindruck von Authentizität durchaus noch 
verstärken, weil dem Zuschauer dadurch bewusst werden kann, wie viel Aufwand und Know-how in die Filmproduktion investiert wurde.

Metatexte wie Rezensionen oder Forendiskussionen über einen Film können die Authentisierungsstrategien des Filmtextes noch viel eher unterwandern als Paratexte. Im Unterschied zu Making Ofs, die das Können der Filmemacher betonen sollen, wird bei Metatexten oftmals das Können der Filmemacher infrage gestellt. Seit der Etablierung des Internets ist es für Zuschauer einfacher als je zuvor geworden, nach diversen Informationen zu einem Film zu recherchieren und so dessen Produktionshintergründe - wesentlich kritischer als in Making Ofs - offenzulegen, was aus Sicht der Filmemacher nicht immer wünschenswert ist.

Eine Metatext-Kategorie, die allein dazu dient, die Inszeniertheit von Filmen offenzulegen und damit potenziell deren Authentisierungsstrategien zu zerstören, sind die sogenannten goofs, auf deutsch „Filmfehler“: Auf der IMDb existiert zu jedem Filmeintrag der Menüpunkt „Goofs“, der noch einmal in Subkategorien wie „Anachronisms“, „Plot holes“, „Factual Errors“ und auch „Errors in geography“ unterteilt ist. Gerade die Kategorie „Errors in geography“ belegt eindrücklich, wie wichtig vielen Zuschauern filmgeografische Authentizität ist. Auf YouTube.com ergibt eine Suche nach „film goofs“ Videoergebnisse wie „Io Historical Movie Mistakes“ oder „Top Io Movies mistakes“, die teilweise über sieben Millionen Aufrufe erhalten. Ganze Webseiten widmen sich ausschließlich dem Aufspüren kleinster Filmfehler. Hierbei nennt die Seite dieseher.de ${ }^{43}$ unter anderem folgende Beispiele:

$\$$ leere Gläser werden voll...

\ Tote leben plötzlich wieder...

\ ein Gegenstand oder eine Person vom Set ist zu sehen...

\ Ein Film spielt in England, man sieht jedoch den Eiffelturm...

\Schauspieler werden falsch genannt, Rollen vertauscht...

3 Allgemeine logische Fehler in der Handlung

§ Falsch recherchierte „Fakten“

Neben ihrer Rolle im Authentizitätsdiskurs spielen Para- und Metatexte eine sehr wichtige Rolle bei der Generierung von Filmtourismus, da die allermeisten Zuschauer erst durch diese Texte die

\footnotetext{
${ }^{43}$ Zugriff: 15. September 2014 .
} 
locations in Erfahrung bringen können. ${ }^{44}$ Dies kann durchaus auch im Rahmen eines nation brandings geschehen, wenn sich - womöglich extra dafür bezahlte - Zuschauer in ihren Rezensionen scheinbar aus eigener Überzeugung sehr positiv über die neuseeländische location äußern und damit touristische „Schleichwerbung“ für Neuseeland betreiben. Obwohl somit Zuschauerrezensionen mit einer gewissen Skepsis begegnet werden sollte, ist doch davon auszugehen, dass die meisten Zuschauer ihre Rezensionen aus eigener Überzeugung schreiben, insbesondere wenn es sich um sehr kritische Rezensionen handelt.

\subsubsection{4 „Sehnsuchtsgrad“, location-Vermittlung, „Referenzialitätsgrad“ als Faktoren bei der} Generierung von Filmtourismus

Sowohl der „Authentizitätsgrad“ eines Films als auch die Verdrängung realer locations durch CG-Landschaften haben direkte Auswirkungen auf das Phänomen des Filmtourismus, was daher in den Analysen auch thematisiert werden soll. Die genauen Faktoren, die Filmtourismus erzeugen, sind nach wie vor nicht genau geklärt, was sich vor allem daran, zeigt, dass einige Filme trotz hoher Erwartungen keinen signifikanten Filmtourismus erzeugen, während andere Filme, von denen man es nicht erwartet hätte, überraschenderweise Scharen von Filmtouristen anlocken. Aufgrund dieser Ungewissheit reagiert die Tourismusindustrie nach wie vor „reaktiv“ und kaum „proaktiv": Selbst im Falle von Lord of the Rings waren viele Touristikunternehmen anfangs von den vielen Anfragen zu Lord of the Rings-spezifischen Touren überfordert, da sie die Nachfrage und deren Umfang nicht antizipiert hatten (Huffstutter, 2003) - Neuseeland vermarktete sich erst dann als „Mittelerde“, als schon längst offensichtlich war, dass erhebliches touristisches Interesse durch den Film geweckt worden war.

Entsprechend schwierig gestaltet sich demnach auch das Ermitteln konkreter Faktoren, mit denen mit hoher Sicherheit prognostiziert werden könnte, ob ein Film Filmtourismus generieren wird oder nicht. Folgende Faktoren werden als möglicherweise in kausalem Zusammenhang mit Filmtourismus stehend erachtet:

\$ kommerzieller Erfolg

\$ kritischer Erfolg bei Zuschauern und Kritikern

\footnotetext{
${ }^{44}$ Nur mit der Geografie bestimmter locations besonders vertraute Zuschauer können unter Umständen auch ohne das Heranziehen von Para-/Metatexten allein durch das Betrachten des Films herausfinden, wo der Film gedreht wurde, insbesondere durch das Erkennen nationalspezifischer Marker wie beispielsweise endemischer Flora und Fauna oder charakteristischer Geomorphologie.
} 
$\$$ „mitreißender“ Plot und Charaktere

§ Grad der Vermarktung der location durch Touristikunternehmen

Dieser Aufzählung Filmtourismus generierender Faktoren möchte ich drei weitere hinzufügen, die vor allem auch im Kontext von filmgeografischer „Authentizität“ und „Entlokalisierung“ erörtert werden sollen: „Sehnsuchtsgrad“, location-Vermittlung und „Referenzialitätsgrad“. Mit „Sehnsuchtsgrad“" ist hier der Grad der Begeisterung der Zuschauer für die dargestellte Filmwelt gemeint. Hiervon untrennbar ist die Einstufung dieser Filmwelt als authentisch oder inauthentisch. Nur wenn Zuschauer die Filmwelt als glaubwürdig erachten, d. h. auch als geografisch kongruent und bei Fantasywelten die Anforderungen der Archaik, Sublimität und des Fotorealismus erfüllt sehen, können sie Immersion empfinden und einen emotionalen Bezug zum Film herstellen. Negative Extreme bezüglich des Plots oder der visuellen Darstellung sind für die Immersion gleichermaßen hinderlich: Ein Film mit großartigen Effekten, aber „schlechtem“ Plot und Schauspielern dürfte ebenso wenig Filmtourismus auslösen wie ein Film mit überzeugendem Plot und Schauspielern, dafür aber „unterirdischen“ visuellen Effekten, da in beiden Fällen eine tiefere Immersion kaum möglich ist und die Zuschauer den Film auf vielerlei Ebenen als inauthentisch empfinden werden. Stattdessen ist davon auszugehen, dass sowohl visuelle Effekte als auch Plot und Charaktere überzeugend sein müssen.

Tooke und Baker stellen in puncto Filmtourismusgenerierung bei Filmen verschiedener Handlungsort-location-Konstellationen folgende interessante These auf:

It seems that if the film location is the true setting, the visitor visits the location: if the film location represents a fictional setting, the visitors go to the location; but if the film location represents a different actual setting, the visitors go to the place represented (Tooke and Baker 1996: 93). ${ }^{45}$

Diese These erscheint insofern plausibel, als dass Zuschauer bei Filmen der dritten und vierten Simulationsstufe die location womöglich als „falsche“ location einstufen und aufgrund dieser Assoziation mit Qualitäten der „Inauthentizität“ stattdessen das vom Handlungsort repräsentierte Land besuchen wollen, sofern sie der Film trotz empfundener filmgeografischer Inauthentizität dennoch

\footnotetext{
${ }^{45}$ Tooke und Bakers These ist zwar grundsätzlich zuzustimmen, könnte aber in vielerlei Hinsicht noch differenzierter formuliert werden: So ist, wie dargelegt, zu berücksichtigen, ob und wie viele Zuschauer überhaupt Kenntnis von der eigentlichen, „falschen“ location haben. Wenn dies nicht der Fall ist, besteht überhaupt keine Wahlmöglichkeit zwischen „Handlungsort-Tourismus“ und „location-Tourismus“. Des Weiteren ist auch die Art des Handlungsortes in Betracht zu ziehen. Sofern der Handlungsort ein Krisengebiet darstellt oder extrem abgelegen ist, könnte es für interessierte Zuschauer aus Sicherheitsgründen reizvoller sein, die zumeist ungefährliche location zu besuchen und dennoch die „Atmosphäre“ des Handlungsortes erleben zu können.
} 
auf anderen Ebenen anspricht. Tooke und Bakers These wird vor allem bei der Analyse des Films The Waterhorse: Legend of the Deep relevant sein.

Ein zweiter wichtiger Aspekt ist der der Vermittlung. Hiermit ist weniger der Grad der touristischen Vermarktung der location an sich gemeint, sondern vielmehr die überaus wichtige Rolle von Para-/und Metatexten bei der Generierung von Filmtourismus. Die Sehnsucht der Zuschauer nach einem „realen“ Erleben eines Handlungsortes allein kann keinen Filmtourismus auslösen - die Zuschauer müssen auch wissen, mittels welcher location sie ihre Sehnsucht verwirklichen bzw. „kanalisieren“ können. Nur wenn Zuschauer wissen, dass reale locations eingesetzt wurden und um welche locations es sich genau handelt, können sie einen referenziellen Bezug zwischen Handlungsort und location herstellen. Dieser referenzielle Bezug muss demnach klar kommuniziert werden, beispielweise in Making Ofs oder auf Filmdatenbanken wie der IMDb.

Als dritter Aspekt ist der Grad der Referenzialität zu nennen, der vor allem eine Rolle bei der Zufriedenheit der Filmtouristen beim Besuchen der locations spielt, aber auch bei der Abwägung, ob sich der Besuch einer location überhaupt lohnen würde. Hier ist insbesondere die ikonische Form der Referenzialität, d. h. der Grad der Ähnlichkeit zwischen location und Handlungsort, entscheidend. ${ }^{46}$ Roesch und Carl weisen daraufhin, dass die Zufriedenheit der Filmtouristen sehr häufig von einem Wiedererkennungseffekt (2009: I4I; 2004: 148) abhängt: Je mehr in der location visuelle Fragmente des Handlungsortes wiedererkannt werden, desto „hyperrealer“ ist das Erlebnis. Roesch spricht in diesem Zusammenhang von „mental visions“ der Touristen, einem temporären Abtauchen in die Filmwelt am Ort der location, die umso stärker war, desto mehr die location dem Handlungsort der jeweiligen Filmszene ähnelte und folglich diese „Vision“ auf die location projiziert werden konnte (2009: I4I-I44) - so wie es die Filmemacher in gewisser Hinsicht auch schon getan hatten. Hierbei spielt die durch zunehmenden Einsatz digitaler Landschaftselemente und damit einhergehender Entlokalisierung eine große Rolle: Je mehr Landschaftselemente digital generiert sind, desto geringer ist der ikonische Referenzialitätsgrad und desto geringer der Wiedererkennungseffekt beim Besuchen der location. Insofern ist bei ausbleibendem Wiedererkennungseffekt die Enttäuschung der Filmtouristen kaum überraschend, was wiederum darauf hindeutet, dass sich Zuschauer zwar über den generellen Einsatz von CGI im Klaren sind, das Ausmaß aber unterschätzen und folglich auch den Grad der Ikonizität regelmäßig überschätzen. Während die im Hand-

\footnotetext{
${ }^{46}$ Der indexikalische Bezug weist die Zuschauer darauf hin, dass eine reale location abgefilmt worden sein muss, wohingegen dieser indexikalische Bezug bei digitalen Landschaften ja gerade wegfällt (allerdings kann weiterhin ein ikonischer Bezug zu realen Orten auch bei digitalen Landschaften gegeben sein).
} 
lungsort trotz CGI-Einsatz verbliebenen sichtbaren „Reste“ der location für Filmtourismus relevant sind, so verblassen durch immer stärkere digitale Manipulation allmählich die charakteristischen Züge der location. Umso wichtiger wird, wie Carl konstatiert, die Rolle touristischer Guides, die die Touristen auch auf kleinste Reste von Ikonizität in der location hinweisen, um weiterhin die $\mathrm{Zu}$ friedenheit der Touristen zu garantieren (2004: 148), wobei auch Guides eine zu geringe Ikonizität nicht mehr ausgleichen können. Ob die häufig bei digital generierten Landschaften eingesetzten Fototexturen noch ausreichen, um durch (minimale) Ikonizität noch Filmtourismus auslösen zu können, wird eine wichtige Fragestellung bei der Analyse der Filme der 7. Simulationsstufe sein.

\section{3 Überlegungen zu Authentizität, De- und Entlokalisierung in I. +}

\section{Simulationsstufe}

Im Methodikteil dieses Kapitels habe ich filmgeografische Authentizität als Aushandlungsprozess zwischen Filmemachern und Zuschauern definiert. Die Frage nach der Authentizität filmischer Handlungsorte lässt sich demnach nicht abgekoppelt von der Rezipientensicht adäquat bestimmen. Die Zuschauer äußern sich in ihren Rezensionen und Forenbesprechungen zu neuseeländischen Filmen der I. und 2. Simulationsstufe nicht zu Fragen der filmgeografischen Authentizität dieser Filme. Dies ist vor allem dadurch zu erklären, dass sich ohne eine offensichtliche, d.h. z. B. durch Paratexte ermittelbare Inkongruenz zwischen Handlungsort und location, wie dies bei Filmen der 3. und 4. Simulationsstufe der Fall ist, diese Fragen für den Zuschauer gar nicht erst stellen und somit auch nicht als "Problem" wahrgenommen werden. Gerade auch weil The Piano und Whale Rider den Zuschauern keinen Anlass gaben, die "Authentizität” der Darstellungen der neuseeländischen Landschaft infrage zu stellen, konnten diese Filme Filmtourismus auslösen. ${ }^{47}$

Allerdings besteht aber selbst bei Filmen der I. und 2. Simulationsstufe eine grundlegende geografische Inkongruenz in der Form, als dass es sich bei einer filmischen Darstellung neuseeländischer Geografie immer um eine virtuelle, semiotisch codierte Geografie handelt, die über ein Medium vermittelt wird und somit nie identisch sein kann mit der materiellen, physisch existenten Geografie des realen Neuseelands. Allerdings ist diese Diskrepanz nicht ohne weiteres als "inauthentisch” zu werten, da sich wie erwähnt (In-)Authentizität nicht entkoppelt von der Rezipientensicht bestimmen lässt. Nach dem in dieser Arbeit zugrunde gelegten Verständnis von Authentizität gibt

\footnotetext{
${ }^{47}$ Zweifelsohne ist dies nicht der einzige Grund für ein film tourism inducement dieser Filme gewesen. Leotta weist unter anderem auf den kritischen und kommerziellen Erfolg der Filme sowie auf deren stilisierte Landschaftsdarstellung hin, die auch durch ihren Fokus auf den Strand und das Meer einen virtuellen tourist gaze bedienen (2011: 104-IO5, 132-I33).
} 
es keine intrinsische, objektiv bestimmbare Authentizität. De-/Entlokalisierung hingegen ist als Voraussetzung und nicht etwa als Folge der Wertung als filmgeografisch (in-)authentisch einzustufen. Daher ließe sich an dieser Stelle fragen, inwiefern auch bei Filmen der I. und 2. Simulationsstufe von einer De- oder Entlokalisierung der location gesprochen werden könnte, auch wenn dies bei Zuschauern keine Fragen nach filmgeografischer Authentizität aufwirft.

In Filmen der I. und 2. Simulationsstufe lassen sich zwei Darstellungsstrategien ermitteln, die als besondere Formen der Delokalisierung definiert werden könnten: Zum einen die Simulation einer neuseeländischen Region durch eine andere neuseeländische Region, zum anderen eine bewusste “Anonymisierung” des neuseeländischen Handlungsortes. Ein Beispiel für die erstgenannte, "intranationale" Form der Delokalisierung ist der Film Out of the Blue. Während der Film Bad Blood auch tatsächlich in der Kleinstadt Hokitika, wo Stanley Graham 194I ein Massaker anrichtete, gedreht werden konnte, mussten die Filmemacher von Out of the Blue bei der Darstellung des Settings Kompromisse eingehen, da sich einige Bewohner Aramoanas gegen einen Dreh vor Ort aussprachen. Letztendlich wurde der Großteil des Films nicht in Aramoana, sondern in der sechs Kilometer entfernten Ortschaft Long Beach gedreht. Zudem bestanden die Bewohner darauf, dass der Film nicht den Titel "Aramoana” tragen solle. Dieser Einwand beruht höchstwahrscheinlich auf der Befürchtung, dass der Titel “Aramoana” den konkreten Ort des Traumas, der zum Zeitpunkt des Massakers von den Nachrichtenmedien bereits popularisiert worden war, einmal mehr unmittelbar für den Zuschauer lokalisierbar machen würde und eine pietätlose, sensationalistische Verwertung der tragischen Ereignisse zu kommerziellen Zwecken darstelle. ${ }^{48}$ Out of the Blue ist somit ein Beispiel für eine Delokalisierung, bei der nicht ein ausländischer Handlungsort durch eine neuseeländische location simuliert wird, sondern auf lokaler Ebene eine neuseeländische Region durch eine andere neuseeländische Region delokalisiert wird. Auch bei der Produktion von Illustrious Energy wurde von den Produzenten in Erwägung gezogen, das charakteristische Otago-Hochland durch die sogenannten Bombay Hills nahe Auckland simulieren zu lassen, wogegen sich jedoch der Regisseur Leon Narbey sperrte, da dies seinen Vorstellungen einer authentischen Darstellung des Handlungsortes widersprochen hätte (Narbey, 20II). Illustrious Energy und Out of the Blue illustrieren, dass es trotz der grundsätzlichen Übereinstimmung von Handlungsort

\footnotetext{
${ }^{48}$ Dass diese Bedenken nicht unbegründet sind, beweist das Schicksal der australischen Kleinstadt Snowtown, die nach einer Mordserie aufgrund der Darstellung in den Medien ungewollte Popularität erlangte und einen regelrechten Besucheransturm erlebte. Aufgrund dieses Dark Tourism zog die Stadt in Erwägung, zum Zeitpunkt der Ausstrahlung des Spielfilms The Snowtown Murders (2OII), sich in "Rosetown" umzubenennen, um einem durch den Film ausgelösten Ansturm von Filmtouristen zumindest zeitweise zu entgehen.
} 
und location in Filmen der I. und 2. Simulationsstufe filmgeografische Inkongruenzen geben kann, die den Authentisierungsstrategien der Filmemacher widersprechen. Diese filmgeografischen Inkongruenzen sind allerdings von den Zuschauern nicht weiter problematisiert worden, da diese intranationalen Inkongruenzen kein derart offensichtliches Aneinanderreiben wie das zweier unterschiedlicher nationaler Räume darstellen.

Die zweite Form der Delokalisierung, die Anonymisierung des neuseeländischen Handlungsortes, ist dann gegeben, wenn der Handlungsort von den Filmemachern bewusst derart schwach spezifiziert ist, dass die Zuschauer den Handlungsort weder durch eindeutige Landschaftsmarker noch mittels paratextueller Hintergrundinformationen (z. B. Infotext auf dem DVD-Cover) eindeutig lokalisieren und als „neuseeländisch“ identifizieren können. Im Gegensatz zur Delokalisierung in Filmen der 3. bis 4. Simulationsstufe, wo der Handlungsort explizit als nicht-neuseeländisch, d.h. als Ausland oder Fantasywelt dargestellt wird, handelt es sich auch hier wieder um eine Form der Delokalisierung, die für die Zuschauer nicht zu Fragen bezüglich der Authentizität dieser Filme geführt hat.

Der Handlungsort von Vincent Wards Vigil zeichnet sich durch seinen „Schwellencharakter“ (Liminalität) aus: Die sehr abgelegene Region erscheint wie ein Hybrid aus Weideland/ Kulturlandschaft und Naturlandschaft/Wildnis, zwischen Natur und Zivilisation. Interessanterweise zeichnet sich der Film aber trotz Wards aufwendiger Suche nach einer spezifischen location durch eine „Unspezifizität“ der Landschaft aus. Lynette Read bezeichnet Vigil dennoch als Wards „most specifically New Zealand film“ und begründet dies mit dem Verweis auf die typischen schweigsamen Farmer-Charaktere, die allgegenwärtigen Schafe, den Traktor und das isolierte Setting (2004: 208). Meines Ermessens sind dies jedoch genau die Aspekte, die als nationaltypische aber nicht nationalspezifische Marker einzustufen sind, da die genannten Aspekte ebenso für andere Länder wie beispielsweise Schottland, Irland oder auch Island zutreffen können. Vielmehr ist Duncan Petrie zuzustimmen, wenn er in Shot in New Zealand über Vigils Kameramann Alun Bollinger schreibt: „[...] Bollinger came up with a predominant blue/grey, almost monochromatic look, which, along with the setting and the production and costume design, serves to detach the drama from any specific sense of place or time“ (2007: 93). Ähnlich konstatiert Murray, dass Vigil „spatially and temporally unlocated“ sei (2008: 172). Letztendlich räumt auch Read ein, dass Ward keine spezifisch neuseeländischen Intentionen mit Vigil verfolgte, sondern vielmehr eine höchstpersönliche Vision vermitteln wollte und auch aufgrund seiner starken Beeinflussung durch den 
europäischen Kunstfilm wenig Interesse an der Darstellung einer klar erkennbar neuseeländischen Landschaft hatte. In einem Interview mit Read sagt Ward: „,[I did not] consciously set out to make a New Zealand film. I don't find I easily identify with the style of other films made in New Zealand. [...] I'm looking for pockets of the outside world which match my own interior vision" (2004: 208). Während beispielsweise in The Price of Milk vor allem die Präsenz der Māori die pastorale Landschaft als neuseelandspezifisch markiert, fehlt auch dieser Marker in Vigil. Die ausschließlich weißen Bewohner scheinen sich vielmehr in einem „Niemandsland“ am „Ende der Welt" zu befinden.

Dies steht in Kontrast zu der erkennbar neuseeländischen Landschaftsmarkierung in Filmen wie Race for the Yankee Zephyr, Whale Rider oder auch Wards späterem Film River Queen, wo dem tourist gaze in seiner Suche nach stereotypischer „Neuseeländischkeit“ der Landschaft entgegengekommen wird und sich mit solchen Filmen auch viel eher Filmtourismus generieren lässt. Hiermit soll nicht suggeriert werden, dass es dem Zuschauer nicht möglich sei, das Setting in Vigil als neuseeländisch zu identifizieren. Dies ist zweifelsohne anhand diverser Paratexte (DVD-Cover) oder Metatexte (Rezensionen) möglich. Wichtig ist hier aber die fehlende Markierung der Landschaft an sich, was auf einer bewussten ästhetischen Entscheidung des Regisseurs beruht. Wenn aber der Handlungsort in Vigil sich somit in einer Art semiotischem „Schwebezustand“ befindet, dann kann hier von einer Delokalisierung der neuseeländischen location gesprochen werden, da deren Neuseeländischkeit subtil verschleiert wird.

Ähnlich verhält es sich auch bei dem Horrorfilm The Irrefutable Truth About Demons von Glenn Standring, wobei der Handlungsort hier sogar noch schwächer markiert ist als derjenige in Vigil. Trotz des Drehs in Wellington wurde darauf verzichtet, eindeutig als neuseeländisch erkennbare Marker wie den „Beehive“ (das neuseeländische Parlamentsgebäude) in der filmischen Stadtlandschaft zu positionieren. Da auch die Paratexte wie das DVD-Cover des Films keinerlei Angaben zum Ort des Geschehens machen, ist der Handlungsort für den Zuschauer kaum genau zu lokalisieren. Diese äußerst vage Lokalisierung des Handlungsortes ist allerdings kein „Versehen“, sondern eine bewusste Strategie der Filmemacher gewesen, um durch die bewusste Delokalisierung der location dem Handlungsort ein „internationaleres“ Flair zu verleihen. Dies wird umso deutlicher, wenn man bedenkt, dass Standring generell danach strebt, kommerziell erfolgreiche, ein möglichst breites Publikum ansprechende Filme zu produzieren, die im Gegensatz zu Wards Filmen 
nicht an einem europäischen art house-Stil orientieren, sondern vielmehr einem Hollywoodstil nacheifern.

Diese Orientierung am Hollywoodkino wird auch in Standrings Film Perfect Creature ersichtlich, dem bis heute teuersten lokal in Neuseeland produzierten Film. Mit seiner retrofuturistischen Steampunk-Ästhetik stellt der Handlungsort eine Art alternative Gegenwart dar, in der die Zukunftsszenarien von Schriftstellern wie H. G. Wells oder Jules Verne Realität geworden sind. Die Industrialisierung hat sich nicht in die von moderner Halbleitertechnik und Digitaler Revolution geprägte postmoderne Gesellschaft weiterentwickelt, stattdessen sind die Mechanik und der Dampfantrieb weiterentwickelt worden. Im Film schweben daher Zeppeline in einem „alternativen“ Neuseeland („nuovo zelandia“) des Jahres 1969 durch die Lüfte. Die Gebäudearchitektur stellt eine Mischung aus viktorianischer (Neo-)Gotik und Baustilen der 1940er-Jahre dar. Verkompliziert wird die Lokalisierung des Handlungsortes durch die Bezeichnung der Stadt als „Jamestown Pacific Colony“, was auf die amerikanische Gründungskolonie hinweist und einen postkolonialen Hintergrund des Settings suggeriert.

Obwohl die vom neogotischen Stil geprägten neuseeländischen Städte Dunedin und Oamaru als locations dienten, erscheint das Setting als eine bizarre Mischung aus viktorianischem London, angereichert durch neuseelandspezifische Artefakte Dunedins und Oamarus. Der seit Lord of the Rings deutlich verstärkte Globalisierungseinfluss auf neuseeländische Filme ist hier besonders ersichtlich, da die urban Gothic-Ästhetik in Perfect Creature vielmehr europäischen und amerikanischen Konventionen folgt und somit nicht mehr als nationalspezifischer Ausdruck eines kiwi Gotbic, d. h. einer glokalisierenden Aneignung einer ausländischen Ästhetik, angesehen werden kann. Der intensive Einsatz von CGI verfremdet die neuseeländische location stark und verleiht dem Film eine für viele postmoderne Vampirfilme wie Twilight oder Underworld typische „musikvideoartige“, stark stilisierte Ästhetik. Durch den Einsatz von CGI, der zweifellos durch die Lord of the Rings-Trilogie motiviert ist, kann hier nicht nur von einer Delokalisierung, sondern auch von einer partiellen Entlokalisierung der neuseeländischen location gesprochen werden, allerdings in immer noch weitaus geringerem Ausmaß als in Filmen der 5. oder gar 7. Simulationsstufe.

Dennoch können laut Cameron, der den Handlungsort des Films als heterotypischen Raum bezeichnet, zumindest lokale Bewohner Dunedins und Oamarus durchaus noch einige Gegenden und Gebäude wiedererkennen (2010: 67). Diese geografischen „Spezialkenntnisse“ der lokalen Bevölkerung gehen aber bei einem globalen Publikum unter, wo dieser Wiedererkennungsef- 
fekt nicht gegeben ist. Perfect Creature vermittelt somit aufgrund des futuristischen Settings und des CGI-Einsatzes ein filmgeografisch stark verzerrtes Bild der zugrundeliegenden neuseeländischen locations. Obwohl der Film noch der I. Simulationsstufe zugeordnet werden kann, driften Handlungsort und location recht stark auseinander und nähern sich damit Filmen der 3. Simulationsstufe an, wo Handlungsort und location eindeutig auseinanderdriften. Cameron ist allerdings zuzustimmen, wenn er argumentiert, dass das Nationale in solchen Filmen nicht gänzlich verschwindet und daher noch nicht von einem „post-nationalen“ Film gesprochen werden sollte, da das Neuseelandspezifische immer noch auf einer Ebene vorhanden ist und mit anderen Ebenen wie denen des Englischen und der Fantasy interagiert. Cameron schreibt: „Tampering with the genetic/generic code of New Zealand horror, these films have pushed the well-established national Gothic into uncertain new areas“ (70), d. h. der neuseelandspezifische kiwi Gothic-Stil ist hier deutlich von transnationalen Gothic-Elementen ,verwässert“. Letztendlich kommt Cameron zu dem Schluss, dass Filme wie Perfect Creature „neither New Zealand films nor foreign films“ seien (68), also quasi auch hinsichtlich ihrer Handlungsorte als national-ausländische Hybride eingestuft werden sollten. Gerade dieses „Weder-Noch“ solcher Handlungsorte beschreibt die für die genannten Filme typische Form der Delokalisierung, die aber nicht so weit reicht wie bei Filmen der 3. und 4. Simulationsstufe.

Bei den hier erörterten Filmen der I. und 2. Simulationsstufe kann somit durchaus von Deund Entlokalisierung gesprochen werden, jedoch nicht in dem Ausmaß, wie dies bei Filmen der 3. bis 7. Simulationsstufe der Fall ist. Vor allem aber tritt erst bei den im Folgenden zu analysierenden Filmen das Problem filmgeografischer Inkongruenz derart deutlich zutage, dass dies bei den Rezipienten Fragen nach der Authentizität dieser Filme aufwirft, was wiederum filmtouristische Folgen hat. Die zuerst zu analysierenden Filme der 3. und 4. Simulationsstufe werden zusammen analysiert, da sie sich zwar durch ihren zeitlichen Bezug unterscheiden, durch ihre realitätsbasierten Handlungsorte aber immer noch so viele Gemeinsamkeiten bezüglich der Authentisierungsstrategien der Filmemacher aufweisen, dass eine getrennte Analyse nicht sinnvoll erscheint.

\subsection{Analyse: III. und IV. Simulationsstufe 3.4.I Vertical Limit (2000)}

Bei Vertical Limit handelt es sich um einen am K2 im Karakorum-Gebirge abspielenden, actionlastigen Abenteuerfilm der dritten Simulationsstufe. Der Film reiht sich durch sein Gebirgssetting, die Abenteuernarrativik, die Mensch-gegen-Natur-Thematik und die Betonung sublimer 
Landschaftsaspekte in die Tradition früherer Bergfilme wie denen von Luis Trenker und modernen Hollywood-Produktionen wie Cliffhanger (1993) ein. Trotz des in Neuseeland geborenen Regisseurs Martin Campbell ist Vertical Limit eine insgesamt stark transnationale Produktion, da der Großteil der Filmmitarbeiter ausländisch ist und keine neuseeländische Produktionsfirma involviert gewesen ist. Der Film fällt vom Produktionszeitpunkt her mit dem Jahr 1999 in eine für ausländische Produzenten vergleichsweise „anreizarme Zeit“, da das „Steuerloch“ für Filmproduktionen in Neuseeland bereits 1984/5 - wie im ersten Kapitel erläutert - geschlossen wurde, der neuseeländische Large Budget Screen Production Grant für ausländische Produktionen aber erst seit 2004 wieder stärkere finanzielle Anreize geben würde. Dennoch gab es auch im Zeitraum 19852004 noch genügend Anreize, die einen Filmdreh in Neuseeland lohnenswert erscheinen ließen. In der vom New Zealand Screen Council herausgegebenen Broschüre „Overview of the New Zealand Screen Production Sector, November 2005“ werden die internationale Anerkennung des Talents und zunehmende Bandbreite an Kompetenzen der neuseeländischen Filmindustrie als Gründe genannt, warum sich die Filmemacher von Vertical Limit, Last Samurai und anderer Filme für einen Dreh in Neuseeland entschieden haben und somit als runaway-Produktionen eingestuft werden können (2005: 12).

Obwohl somit primär pragmatische und ökonomische Erwägungen dazu geführt haben dürften, Vertical Limit in den neuseeländischen Südalpen statt im Karakorum zu drehen, ${ }^{49}$ betonen die Filmemacher im Making Of „Surviving the Limit“ immer wieder, wie viel Wert auf Authentizität gelegt worden sei, um ihr Filmprodukt nicht als bloße economic runaway, sondern auch als eine creative runaway production ${ }^{50}$ vermarkten zu können. Der Handlungsort in Vertical Limit wird explizit markiert (u. a. mittels des eingeblendeten Paratextes „Lower Himalaya, Pakistan“ [0o:07:50]) und viel Aufwand investiert, den Handlungsort glaubwürdig zu inszenieren. Zu den eingesetzten Authentisierungsstrategien gehören die der Projektion, der Autorisierung und der Kompensation: Wenn die Filmemacher die visuelle und „charakterliche“ Ähnlichkeit zwischen dem neuseeländischen Mount Cook und dem K2 betonen („Mount Cook, a worthy stand-in for

\footnotetext{
${ }^{49}$ Ein tatsächlicher Filmdreh im Karakorum-Gebirge und das Besteigen des K2 wäre extrem gefährlich gewesen und hätte die Filmemacher aufgrund der Unzugänglichkeit des Geländes vor kaum zu bewältigende logistische Probleme gestellt. Die neuseeländischen Südalpen hingegen sind schon aufgrund ihrer Beliebtheit bei Touristen vergleichsweise gut erschlossen. ${ }^{5}$ Sogenannte creative bzw. artistic runaways stellen eine Unterkategorie der runaways dar, bei denen nicht ökonomische oder pragmatische, sondern primär kreative Gründe für einen Dreh außerhalb der USA sprechen. Ein Beispiel wäre ein Film, der in Paris spielen soll und aus Authentizitätserwägungen tatsächlich in Paris gedreht wird, statt Paris im Filmstudio oder einer US-Stadt nachzubilden. Reine creative runaways nach Neuseeland sind allerdings selten und im vorliegenden, zu analysierenden Filmkorpus nicht gegeben. Hauptgrund dürfte die geringe Nachfrage nach einem explizit neuseeländischen Handlungsort in Hollywoodfilmen sein (im Gegensatz beispielsweise zum beliebten Paris).
} 
the deadly $\mathrm{K}_{2}$ can be just as treacherous for those prepared to take their endeavor to the vertical limit“ („Surviving the Limit“ [oo:18:16-0o:18:29]) stellt dies keine objektive Feststellung, sondern vielmehr eine subjektive Wertung dar. Da der Regisseur nicht darauf verweist, dass die Ähnlichkeit der beiden Berge lediglich seinen persönlichen Eindruck widerspiegelt (beispielsweise durch Zusätze wie „in my opinion“), sondern in der Aussage ein Objektivitätsanspruch zu erkennen ist, stellt dies eine Projektion der eigenen Sichtweise auf die Allgemeinheit und damit auch auf die Zuschauer dar, als eine Art Selbstlegitimation für die nicht zu leugnende filmgeografische Inkongruenz im Film. Mit anderen Worten hofft der Regisseur, dass der Zuschauer die Legitimität der Südalpen als „Double“ für den tausende Kilometer von der location entfernten Karakorum anerkennt und die Delokalisierung überzeugend finden wird.

Der Extrembergsteiger Ed Viesturs, der sowohl als filmischer Berater mitgewirkt als auch in einer Nebenrolle im Film mitgespielt hat, betont ebenfalls die vermeintlich „objektive“ Ähnlichkeit der beiden geografisch eigentlich weit auseinanderliegenden Gebiete, um dadurch den Handlungsort zu authentisieren: „The glaciers are as big, the crevasses are as deep, the terrain is as steep, the weather is as bad and the scenery is as beautiful. So it's a very good location to shoot a film like this" („Surviving the Limit“ [oo:18:29-0o:I8:43]). Die Einbeziehung eines Profibergsteigers, der, ähnlich wie Reinhold Messner, alle Achttausender und somit auch den $\mathrm{K}_{2}$ bestiegen hat, kann als Versuch der Filmproduzenten angesehen werden, sowohl den Handlungsort als auch das Agieren der Charaktere als „professionelle“ Bergsteiger durch einen anerkannten Fachmann als realistisch und glaubwürdig autorisieren zu lassen. Zwar spielt Viesturs nur eine kleine Rolle im Film, aber allein seine Präsenz und seine Interaktion mit den restlichen Charakteren soll insbesondere Zuschauern, die selbst am Bergsteigen interessiert sind und denen Viesturs ein Begriff ist, eine Aura des „Professionellen" vermittelt werden.

Da aber selbst Projektion und Autorisierung nicht ohne weiteres über die grundlegende filmgeografische Inkongruenz hinwegtäuschen können, wird als dritte Strategie die der Kompensation eingesetzt: Statt im Making Of allein auf die dramatischsten Filmszenen zu fokussieren, die allesamt in einem Filmstudio gedreht worden sind, betonen die Filmemacher vor allem, wie viel Aufwand der Dreh der übrigen on location-Szenen erfordert hat:

We chose to film in a real environment. Up on the mountains with our actors going into situations that actors don't usually go. [...] the shots that we have put our actors, put our story firmly in an alpine location and no amounts of computer generated or visual effects can 
ever really capture the same feeling and emotion („Surviving the Limit“ [oo:06:4500:07:18]).

Das Making Of dient somit nicht in erster Linie dazu, dem Zuschauer zu zeigen, mit welchen „Tricks“ gearbeitet worden ist, sondern vielmehr, um zu betonen, wie viel des filmischen Geschehens „echt“ ist, damit sich der Zuschauer mittels dieses paratextuellen Hintergrundwissens erst recht in das filmische Geschehen hineinversetzen kann. Eine Kompensation stellt dies insofern dar, als dass suggeriert werden soll, dass es zwar nicht das „echte“ Karakorum-Gebirge ist, in dem gedreht wurde, dass die „falschen“ neuseeländischen Berge aber immerhin real sind.

Eine zweite Kompensationsstrategie stellt das digitale Einfügen des echten $\mathrm{K}_{2}$ in die neuseeländische Südalpenszenerie dar (Vertical Limit, Audiokommentar [oo:I3:57]), wie Abbildung 3.8 illustriert.

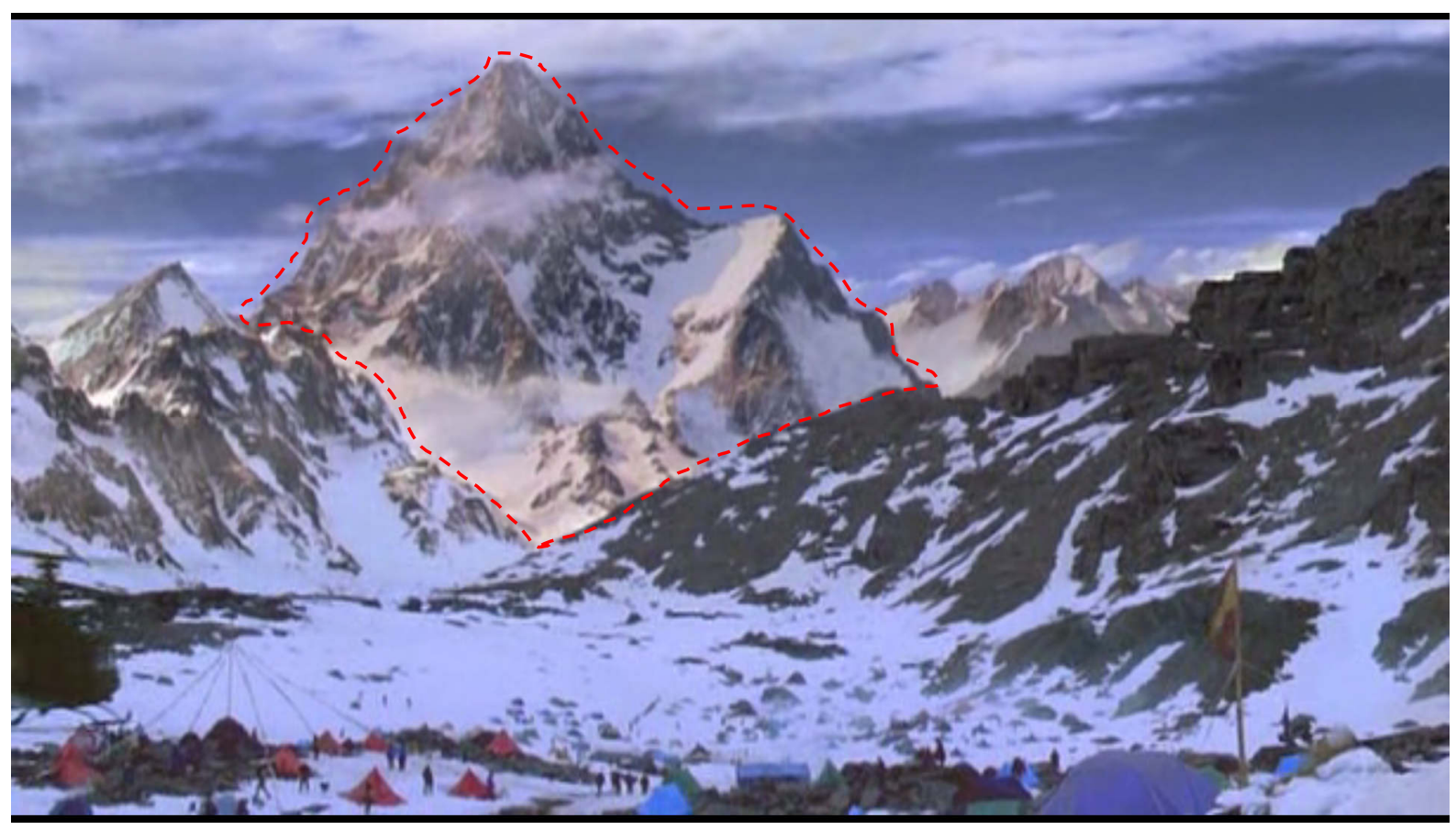

Abbildung 3.8: Südalpenszenerie mit digital eingefügtem K2 (rot eingerahmt) [oo:13:57]

Trotz des Drehs auf realen Bergen und ihrer vorgeblichen Ähnlichkeit mit dem KarakorumGebirge haben es die Filmemacher dennoch als notwendig erachtet, als Zugeständnis an die filmgeografische Authentizität den echten K2 zumindest scheinbar in den Filmdreh miteinzubeziehen. Dieser Prozess stellt eine Befüllung der neuseeländischen location mit einem auslandsspezifischen Marker, in diesem Fall dem „karakorumspezifischen“ K2-Berg dar, dessen charakteristische Form von vielen Zuschauern erkannt werden kann (siehe rote Einrahmung in Abbildung 3.8). Die Funk- 
tion des K2-Berges liegt darin, den Zuschauer dazu zu bewegen, vom Speziellen zum Allgemeinen zu schließen, d. h. bei dem Erblicken des $\mathrm{K}_{2}$ automatisch davon auszugehen, dass es sich auch bei der umgebenden Landschaft tatsächlich um den Karakorum handelt. Es wird auch ersichtlich, dass höchster Wert auf das nahtlose Verschmelzen der verschiedenen, digital zusammengefügten Bildebenen zu einem homogenen Raum gelegt wurde, da kaum zu erkennen ist, dass in dieser Szene zwei weit auseinanderliegende Geografien künstlich miteinander kombiniert worden sind.

Trotz aller eingesetzten Authentisierungsstrategien ist Vertical Limit letztendlich von sehr vielen Zuschauern als in vielerlei Hinsicht extrem inauthentisch eingestuft worden. Neben diversen Kritiken an dem als unrealistisch erachteten Verhalten der Bergsteiger, plot boles und als völlig übertrieben eingestufter Actionszenen wird auch die Gestaltung des Handlungsortes quasi als krönende Form der Inauthentizität gewertet. So schreibt „Rhea“ aus Kalifornien: „The facts were frighteningly inaccurate, and it wasn't even the correct mountain!“ (I. Dezember 2002). Hierbei illustriert das Wort „correct“, dass für viele Zuschauer nur ein Filmen an Originalschauplätzen, in diesem Falle also im Karakorum, als authentisch eingestuft worden wäre, wohingegen der Einsatz anderer locations als „Double“ kategorisch als „incorrect“, also inauthentisch, eingestuft wird. Ein verwandter Kritikpunkt ist der Einsatz von CGI und dem greenscreen-Verfahren. ${ }^{5 \mathrm{I}}$ Hierbei wird nicht der Einsatz von Studioaufnahmen und CGI an sich kritisiert, sondern eine als derart offensichtlich und unrealistisch eingestufte Umsetzung dieser Verfahren, dass digitalisierte und real abgefilmte Raumelemente nicht zu einem homogenen Raum verschmelzen, sondern als getrennte Ebenen wahrgenommen werden und die daraus resultierende „Studioatmosphäre“ ein emotionales Eintauchen in die Bergwelt für viele Zuschauer verhindert.

Die vielen ausgesprochen heftigen und emotionalen Negativ-Kritiken zum Film lassen sich meines Ermessens unter anderen damit erklären, dass Zuschauer gerade bei Filmen über Geschehnisse in kaum zugänglichen Regionen besonderen Wert auf filmgeografische Authentizität Wert legen, weil die meisten diese Regionen nie als Touristen besuchen werden und daher Filme wie Vertical Limit die einzige Möglichkeit darstellen, diese Regionen zumindest im Rahmen eines virtuellen Tourismus bzw. armchair travel zu erleben. ${ }^{52}$ Maximale Authentizität soll quasi als Surro-

\footnotetext{
${ }^{51}$ Mit dem green- bzw. bluescreen-Verfahren kann die Illusion erweckt werden, dass sich eine Person auf einer location befindet, beispielsweise auf einer Alpenwiese, während diese Person tatsächlich nur vor einer grün oder blau leuchtenden Wand in einem Filmstudio agiert, auf die in der Postproduction eine real abgefilmte oder digital generierte Landschaft hineinprojiziert wird und hierbei die Umrisse der Schauspieler ausgespart werden (Richter 2008: 79).

${ }_{52}$ Zwar können unzugängliche Gebiete auch anhand von Dokumentarfilmen „bereist“ werden, allerdings handelt es sich dabei um einen Rezeptionsmodus, der sich deutlich von dem von Spielfilmen unterscheidet und unter anderem meist weniger emotional und „nüchterner“ erlebt wird.
} 
gat für das Versäumnis dienen, diese Gebiete niemals wirklich bereist zu haben. Umso enttäuschender ist es für die Zuschauer, wenn der medial vermittelte Karakorum stark verzerrt dargestellt wird und daher weder als geografische Informationsquelle oder noch als Mittel zur einer „realistischen" geografischen Imagination dienen kann. Insofern können und wollen solche Zuschauer die neuseeländischen Südalpen nicht als „Ersatz-Karakorum“ akzeptieren.

Was zudem der oben genannten Autorisierungsstrategie der Filmemacher geradezu diametral entgegensteht, ist die Reaktion des wohl bekanntesten Bergsteigers Reinhold Messner auf den Film. In der FAZ kritisiert er sowohl den Handlungsort des Films als auch das unrealistische Verhalten der Charaktere:

So wenig die Berge um den Mount Cook aussehen wie der Himalaja, so wenig verhalten sich Menschen im Grenzbereich zwischen Leben und Tod wie in diesem Film. [...] Trotz des großen Aufwands aber ist es nicht gelungen, die Handlung realistisch erscheinen zu lassen. Fast nichts in diesem Film sieht aus wie am wirklichen Berg. Ob mit einer Sprengladung eine Lawine ausgelöst wird oder nach einer Explosion der halbe Berg in die Luft fliegt - es ist zum Lachen. [...] Den Bildern fehlt die Atmosphäre extremer Höhen. [...] Wie Ed Viesturs, ein US-Star-Bergsteiger, der hinter der Kamera als Berater fungierte, so viel Unfug durchgehen lassen konnte, bleibt mir ein Rätsel (Messner, 26. Januar 20oI).

Durch diesen Gegendiskurs, der zudem von einer anerkannten Autorität stammt, wird die Autorisierungsstrategie der Filmemacher nahezu völlig entwertet und dürfte zudem viele Zuschauer in ihrem vernichtenden Urteil über den Film beeinflusst haben. Gleichzeitig tut Messner Bergfilme aber nicht einfach als irrelevante Fiktion ab, sondern misst ihnen durchaus das Potenzial zu, realen (Fehl-)Informationswert zu haben.

Ein wichtiger Grund, warum der Film nicht als authentisch wahrgenommen worden ist, ist der Versuch der Filmemacher, eine Balance zwischen Realismus und Entertainment zu finden. Von dem Bestreben, den Zuschauer einfach zu unterhalten, zeugen die vielen geradezu komödiantischen Einlagen, aber auch Actionszenen, die ganz offensichtlich übertrieben sind und gerade deshalb einen cinema of attractions-Zuschauertyp besonders ansprechen können. Insofern zeigt diese Gruppe auch ein wesentlich entspannteres Verhältnis zu filmischer Authentizität. Trotz seines als unlogisch empfundenen Plots wirkt der Film auf viele Zuschauer allein aufgrund der visuellen Reize als immersiv, während die Frage nach der Authentizität des Handlungsortes in den Hintergrund rückt. User „ichwan_mil“ bezieht sich ganz konkret auf die Authentizitätsdiskurse anderer Rezen- 
senten: „I can't understand some people smack this movie for the sake of 'authenticity'. It is just a movie, it's there for the sake of entertainment. If you absolutely long for authenticity, look for documentary ones instead“ (30. Oktober 20I0). Dies illustriert, dass es Zuschauer gibt, die den Film als durchaus inauthentisch wahrnehmen, aber schlicht kein Bedürfnis an filmgeografischer Authentizität haben und dies für sie keine Voraussetzung für das Eintauchen in die Filmwelt darstellt. Der Hinweis auf Dokumentarfilme zeigt überdies, dass von Rezensenten wie „ichwan_mil“ Dokumentarfilme als besonders authentisches Filmformat und quasi als Gegenentwurf zum als „inhärent inauthentisch" eingestuften Spielfilmformat erachtet wird. Tatsächlich sind die Authentizitätserwartungen an Dokumentarfilme zumindest in filmgeografischer Hinsicht weitaus höher: So würde ein Dokumentarfilm über den K2, der aber wie Vertical Limit aus Sicherheitsgründen am Mount Cook gedreht würde, kaum von den Zuschauern angenommen werden.

Zusammenfassend lässt sich also feststellen, dass ein erheblicher Teil der Zuschauer den Film als inauthentisch wahrgenommen hat und daher die Authentisierungsstrategien der Filmemacher nur eine geringe Wirkung gezeigt haben, wobei sowohl die neuseeländische location als auch die Studioaufnahmen mit CGI als de-authentisierende Elemente maßgeblich zu dieser Wertung beigetragen haben. Trotz dieser „negativen“ Funktion der neuseeländischen location scheint der Film durchaus filmtouristisches Interesse bei einigen neuseeländischen Zuschauern geweckt zu haben. Obwohl keine genauen Daten darüber vorliegen, ob Vertical Limit konkreten Filmtourismus ausgelöst hat, illustrieren Rezensionen wie die von „kid_dynamo“ aus Auckland, dass der Film trotz der lediglich indirekten Vermittlung der neuseeländischen Landschaft das Potenzial dazu besitzt: „I am a New Zealander so yes i am biast. I didn't even know some of this spectacular scenery was in my own back yard. Must go exploring“ (26. Dezember 200o). Die Darstellung der sublimen Südalpen wird vom Rezensenten als Aufforderung verstanden, die eigene Landschaft touristisch zu erforschen. Das neuseeländische Unternehmen Nomad Safaris in Queenstown bietet neben den obligatorischen Touren zu den locations von Lord of the Rings aus diesem Grunde auch eine halbtägige Tour zu locations anderer in Neuseeland gedrehter Filme an, darunter auch zu denen von Vertical Limit („Set jetting“).

\subsubsection{Last Samurai (2003)}

Bei der stark transnationalen Hollywood-Produktion Last Samurai handelt es sich im Gegensatz zu Vertical Limit um einen Film der vierten Simulationsstufe, da sich das Geschehen nicht 
in der Gegenwart, sondern im Japan des 19. Jahrhunderts abspielt und somit auch die Landschaft als eine „historische“ dargestellt werden muss. Tom Cruise spielt hierbei die Rolle des Nathan Algren, der mehrfach die Fronten zwischen „Zivilisation“ (modernisiertes, urbanisiertes Japan) und archaischem Raum (traditionelle Samurai in abgelegener Region) wechselt. Im Vergleich zu dem reinen Bergfilm Vertical Limit wird in Last Samurai aber eine größere Bandbreite an Landschaften und Räumen dargestellt, von urbanen Schauplätzen über Weide-/Steppenlandschaft bis hin zu Wäldern und Bergen. Aber nicht nur der Handlungsort, sondern auch die locations sind in nationaler Hinsicht wesentlich vielfältiger als in Vertical Limit. Statt ausschließlich in Neuseeland zu drehen, wurde auch in Japan und in den USA (dort allerdings primär in Filmstudios) gedreht.

Ähnlich wie bei Vertical Limit wurde auch in Last Samurai ein für den Handlungsort ikonischer Berg extra digital in die neuseeländische Landschaft eingefügt - in diesem Fall der Mount Fuji (Last Samurai, Audiokommentar [oo:30:24]) - um die Landschaft als eindeutig erkennbar „japanisch“ zu markieren und beim Zuschauer den Eindruck entstehen zu lassen, dass auch die gesamte Umgebung tatsächlich japanisch sei. Allerdings räumt Regisseur Edward Zwick ein, dass diese Authentisierungsstrategie bei japanischen Zuschauern, die die Geografie ihres eigenen Landes recht gut kennen dürften, problematisch sein könnte. Bezüglich der Szene, als Algren von den Samurai gefangen genommen wird, kommentiert Zwick: „These are passes in New Zealand which in fact are un-Japanese and I think, may be distressing to some Japanese that they think they don't know where these mountains are in Japan“ (Last Samurai, Audiokommentar [oo:30:27oo:30:37]). Der Regisseur nimmt somit antizipierend in Kauf, dass zumindest japanische Zuschauer den Handlungsort als unglaubwürdig empfinden könnten, da sie die den Mount Fuji umgebenden spezifisch neuseeländischen Bergpässe als „un-japanisch“identifizieren und somit die filmgeografische Inkongruenz erkennen könnten.

Andererseits betont Zwick im Making Of wiederholt, wie wichtig ihm die Authentizität des Films gewesen sei und führt an, wie die Filmemacher versucht haben, aus den diversen locations einen homogenen geografischen Raum zu „weben“: „Throughout the film we’re trying to weave construction in Burbank, real locations in Japan and construction in New Zealand, often within the same scene“ (Last Samurai, Audiokommentar [I2:30-I2:44]). Der Einsatz von „real locations in Japan“, die eine (zeitweilige) Kongruenz zwischen Handlungsort und location sicherstellen, stellt eine Kompensation für den Einsatz nicht-japanischer, d. h. neuseeländischer und amerikanischer 
locations dar. So betont er, wie der Dreh an einem realen japanischen Kloster und dem umliegenden Wald dem Film eine Aura des „heart of old Japan“ verleihe:

This is in a place called Engyoji. It's a 9oo year old monastery that we had to reach by taking a funicular. It's above a small town called Himeji in Japan and we all felt that it was somehow the most fitting and respectful place to begin shooting the film, because it has the heart of old Japan in every space, in every piece of wood [...] (Last Samurai, Making Of [00:00:30-00:00:52]).

Durch den Dreh an einem Kloster, das ein Relikt früherer Zeiten darstellt, soll historische Authentizität gewährleistet werden, die auch für den Zuschauer „,in every piece of wood“ des Handlungsortes in der entsprechenden Filmszene spürbar sein soll.

Eine weitere Authentisierungsstrategie stellt die Argumentation Zwicks dar, dass die neuseeländische Naturlandschaft der Taranaki-Region aufgrund ihres unberührten und vormodernen Erscheinungsbildes adäquater zur Simulation des vormodernen Japans sei als das eigentliche, aber von der Modernisierung stark geprägte Japan, insbesondere in der Fuji-Region. So schreibt Jeff Otto in einem Interview mit Zwick über die Produktionsentscheidungen der Filmemacher:

The physical preparations along with the challenge of accurately recreating I9th century Japan would be a daunting task before film was ever to roll. Modern Japan now bears little resemblance to samurai times so they soon wound up in New Zealand. [...] Zwick wanted authenticity not only in the film's sets, but also in his cast members. There would be no Charlton Heston playing a Mexican here (Zwick, 3. Dezember 2003).

Während das Spielen eines Mexikaners durch einen Amerikaner als inauthentisch erachtet wird, wird hingegen die Tatsache, dass Neuseeland Japan „spielt“, als weniger problematisch erachtet. Durch den Einsatz einer vormodern erscheinenden Landschaft soll letztendlich eine Annäherung an historische Authentizität erreicht werden, um damit die fehlende filmgeografische Authentizität zu kompensieren. Indirekt wird also argumentiert, dass das eigentliche, aber modernisierte Japan (von wenigen Standorten wie dem oben erwähnten Kloster abgesehen) nicht authentisch genug sei, um ein historisches Japan zu simulieren.

Als Vorteil erweist sich hierbei der Umstand, dass an historische Handlungsorte ein etwas anderes Authentizitätsverständnis geknüpft ist als an zeitgenössische Handlungsorte: Während beispielsweise beim zeitgenössischen Handlungsort von Vertical Limit die Originalschauplätze im Karakorum-Gebirge theoretisch hätten abgefilmt werden können, um maximale „Authentizität“ 
zu gewährleisten, ist es bei historischen Handlungsorten in den allermeisten Fällen nicht mit einem schlichten Abfilmen der Originalschauplätze getan, da die historischen Landschaften in ihrer ursprünglichen Form streng genommen gar nicht mehr existieren. So notiert Zwick in seinen Produktionsnotizen: „The Japan we created is one of imagination in that it no longer exists“ (Shin 2010: 1069). Durch diesen zeitlichen Abstand vom „Hier und Jetzt“ ist somit immer auch die Notwendigkeit einer Rekonstruktion geknüpft. Bei Filmen mit zeitgenössischen Handlungsorten besteht stets die Möglichkeit, durch Besuch der Originalschauplätze zu überprüfen, wie „authentisch“ oder „,inauthentisch“ der filmische Handlungsort ist. Im Fall von Vertical Limit konnte beispielsweise Reinhold Messner als jemand, der das Karakorum-Gebirge tatsächlich gesehen hat, beurteilen, inwiefern die filmische Inszenierung des Karakorum akkurat ist oder nicht. Bei historischen Landschaften wie dem Japan zur Zeit der Samurai hingegen ist diese Möglichkeit direkter Überprüfbarkeit nicht mehr gegeben - bestenfalls existieren noch historische Fotos, die einen vagen Anhaltspunkt liefern.

Ebenso wie bei Vertical Limit hat es teils deutliche Kritik an der Authentizität des Handlungsortes von Last Samurai gegeben. Eine wichtige Gruppe, in der viele Rezensenten nicht von der Authentizität des Films überzeugt waren, sind japanische Zuschauer, also diejenige Zielgruppe, bei der Zwick die größten Probleme antizipiert hatte (s. o.). So schreibt der Rezensent „t_man_jp“:

I am a Japanese. In this movie I see many false misunderstandings and errors on Japanese culture $[\mathrm{sic}]$. For example, they try to overlap Samurais and Native Americans, which is very difficult for Japanese to comprehend. In one scene, Samurais were fighting in a jungle. We do not have a jungle in Japan (7. Mai 2005).

Neben vielen anderen Zuschauern stört sich auch dieser Rezensent nicht etwa an der Szene mit dem in die neuseeländische Landschaft einkopierten Mt. Fuji, sondern an den zwar in Neuseeland aber nicht in Japan endemischen Riesenfarnen und Palmgewächsen, die er als ,jungle“ interpretiert. Vor allem an der ersten Schlachtszene in einem Wald, in der die japanischen Kaisertruppen vernichtend von den Samurai geschlagen werden und Algren in Gefangenschaft gerät (Last Samurai [ca. 00:22:30-00:30:00]), wird diese filmgeografische „Inauthentizität“ insbesondere für japanische Zuschauer ersichtlich: 


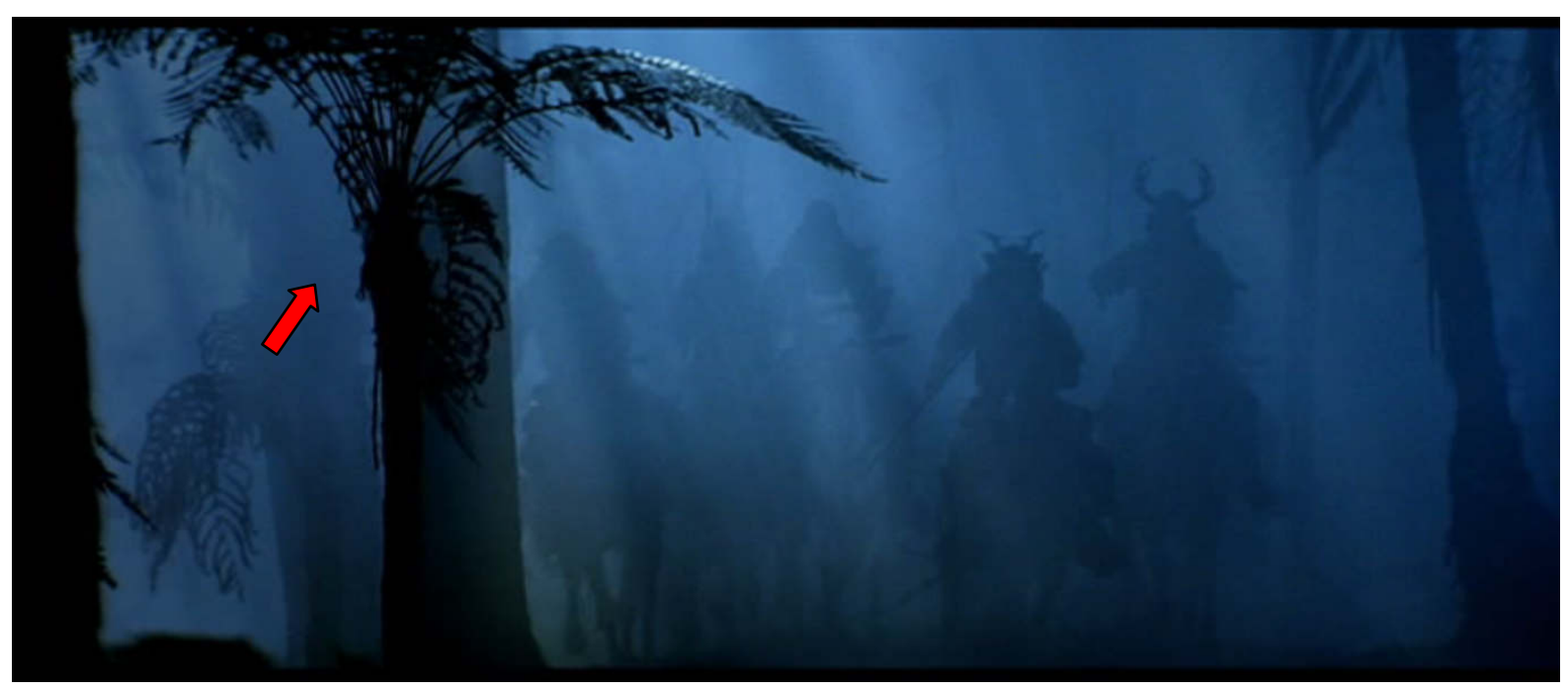

Abbildung 3.9: Angriff der Samurai, mit neuseeländischen Riesenfarnen (roter Pfeil) [00:24:24]

Von den Farngewächsen ist der Silberfarn ähnlich wie der Kiwivogel ein Nationalsymbol Neuseelands, mit der auch die Einzigartigkeit der neuseeländischen Flora und Fauna demonstriert werden soll. Dass die Farngewächse zwecks Filmdrehs nicht aus dem Wald entfernt wurden, um den Wald „japanischer“ erscheinen zu lassen, ist weniger als Nachlässigkeit, sondern vielmehr einem grundlegenden Pragmatismus zuzuschreiben, da, wie im Methodikteil erläutert, die Entleerung der Landschaft von sämtlichen neuseelandspezifischen Landschaftselementen kaum zu bewerkstelligen ist und damit immer auch Indikatoren für eine filmgeografische Inkongruenz erkennbar bleiben. In diesem Fall wurden von vielen Rezensenten ein so kleines geografisches Detail wie die Farne als deutlicher Indikator für die Inauthentizität des Handlungsortes gewertet. Wichtig ist hierbei, dass viele japanische Zuschauer an der Darstellung des Handlungsortes selbst die Inkongruenz erkennen können und dass somit metatextuelle Hintergrundkenntnisse keine unbedingt notwendige Voraussetzung für die filmgeografische Beurteilung eines Filmtextes sind.

Vor allem weil Last Samurai im gleichen Jahr ausgestrahlt wurde wie der letzte Teil der Lord of the Rings-Trilogie (Return of the King), empfanden viele Zuschauer die neuseeländischen locations auch deshalb als inauthentisch, weil diese bereits mit der Fantasywelt „Mittelerde“ aus der Lord of the Rings-Trilogie assoziiert worden sind. So ist die Irritation von „nysalor“ über die gängige Filmpraxis, locations ungeachtet ihrer spezifischen geografischen Charakteristika als nahezu beliebig austauschbar zu behandeln, in seiner Rezension klar ersichtlich: 
The Meiji Restoration is a fascinating period of Japanese history. The Last Samurai examines it with all a finese and insight that can only come of producing it entirely in New zealand. Japan looks a lot like Middle Earth. Come to think of it, the badlands of Little Big Horn look a lot like Middle Earth. No amount of blue tinting can disguise this basic, unsettling visual impairment. In the first battle sequence, you are expecting bands of orcs to come grunting out of the forest. Instead, you get samurai in ${ }^{*}$ medieval ${ }^{*}$ armour. Welcome to I876. Confused? Don't worry, you're not alone („nysalor“, 26. Januar 2004).

An den Rezensionen wird deutlich erkennbar, dass sich die Zuschauer beim Betrachten vieler Szenen in Last Samurai aufgrund bestimmter geografischer Merkmale ständig an Szenen aus Lord of the Rings erinnert fühlen und dies als störend empfinden. Hier ist durch das Wiederverwenden bzw. „Recycling“ bekannter neuseeländischer locations ein semiotischer Konflikt entstanden, bei dem die Zuschauer beispielsweise beim Betrachten grüner neuseeländischer Hügel (=Signifikant) zuerst an „Hobbiton“ (=Signifikat) denken, was die simultane Imagination eines weiteren Signifikats, nämlich „historisches Japan“, erschwert.

Die Wiederverwendbarkeit gleicher oder zumindest sehr ähnlicher locations scheint demnach beschränkt zu sein, insbesondere, wenn sie für Handlungsorte verwendet werden, die zueinander keinerlei Ähnlichkeit aufweisen, wie dies bei Mittelerde und historischem Japan der Fall ist. Dieser Umstand ist insofern wichtig, als dass er auf eine zukünftige Problematik für Neuseeland als location hinweist: Durch Übernutzung von locations für verschiedenste Handlungsorte kann sich ein Abnutzungseffekt einstellen, der Neuseeland mittel- bis langfristig als location uninteressant machen könnte, da Zuschauer immer wieder „neue“, „unverbrauchte“ Geografien sehen wollen.53

Einschränkend muss allerdings angeführt werden, dass es durchaus auch eine kleine Zahl von Rezensenten gibt, für die die vorherige Nutzung Neuseelands als Schauplatz für Mittelerde den japanischen Handlungsort in Last Samurai gerade authentisiert, statt ihn zu de-authentisieren:

Zwick should be praised in the many areas he does succeed in, such as the actual battles. These are close to competing with anything to come out of Middle Earth in the last few years. They are beautifully shot and genuinely exciting. Like Peter Jackson, Zwick uses

\footnotetext{
${ }^{53}$ Dieser Abnutzungseffekt kann auch als eine Erklärung dafür dienen, dass mit jedem neuen Teil der Lord of the Rings- und Hobbit-Trilogie mehr digitale Effekte als im direkten Vorgänger eingesetzt worden sind, um die bereits bekannten Landschaften wieder mit einem Neuigkeitseffekt zu versehen, vor allem, weil bereits im ersten Teil durch die vielen locations, die über ganz Neuseeland verteilt waren, geradezu ein repräsentativer Querschnitt der landschaftlichen Diversität Neuseelands gezeigt wurde und bereits im zweiten Teil der Eindruck entstehen konnte, „alles schon einmal gesehen “ zu haben. Dieser Aspekt wird bei der Analyse der fünften Simulationsstufe näher erörtert werden.
} 
panoramic shots to great effect and even if CGI is being used, you really can't tell. The setting really feels genuine, and therefore it must be commended („psi“, 2I. Januar 2004). Für diesen Zuschauer erscheint der Handlungsort von Last Samurai trotz oder vielmehr gerade aufgrund der vorherigen Nutzung der locations für den als authentisch erachteten Film Lord of the Rings „genuine“. Hierbei sind es vor allem die Kameraführung, die die Sublimität der Landschaft betont sowie der Einsatz fotorealistischer, von abgefilmten realen Landschaftsteilen nicht zu unterscheidender CGI, die beide Filme für den Zuschauer so immersiv machen.

Gerade auch Zuschauer, die sich nicht über die Produktionshintergründe des Film informiert haben, neigen dazu, den Handlungsort von Last Samurai als authentisch einzustufen, weil ihnen die filmgeografische Inkongruenz gar nicht erst auffällt und sie den Film irrtümlicherweise für einen Film der ersten Simulationsstufe halten, bei dem sowohl Handlungsort als auch location japanisch sind. So schreibt Rezensent „Inakaguy“: „(...) worth watching for performances from Ken Watanabe (...) and Japan itself, which turns in a splendid performance of perfect sunsets and verdant mountains“ (22. April 2004). Zwar wurden einige Szenen tatsächlich in Japan gedreht, die Szenen in den Bergen mit den „perfect sunsets“ wurden aber gerade in Neuseeland gedreht. Aufgrund fehlender metatextueller Hintergrundkenntnisse hat dieser Zuschauer somit die Schönheit neuseeländischer Berge irrtümlicherweise mit Japan in Verbindung gebracht. Diese Fehlzuordnung illustriert, dass fehlendes Hintergrundwissen verhindern kann, dass Zuschauer den Film mit Neuseeland (durchaus aber mit Japan) assoziieren und dadurch auch der neuseeländischen Tourismusindustrie ein Nachteil erwächst.

Wie Leotta in seinem Buch Touring the Screen anführt, hat Last Samurai tatsächlich nur kurzzeitig und in geringem Umfang Filmtourismus nach Neuseeland generiert, was unter den Erwartungen der lokalen touristischen Unternehmen lag. Die Erwartung neuseeländischer Tourismusunternehmen, von einem touristischen Spin-Off von Last Samurai zu profitieren, beruhte auf der Ansicht, dass Zuschauer analog zu der Assoziation von Mittelerde mit Neuseeland auch das historische Japan in Last Samurai mit Neuseeland assoziieren würden - dass demnach die neuseeländische location als Projektionsfläche für ein virtuelles historisches Japan anziehender auf Filmtouristen wirken würde, als die reale japanische Landschaft und dass somit Realität an der Simulation gemessen wird und nicht umgekehrt. So wie Lord of the Rings-Filmtouristen die neuseeländischen locations nach Anzeichen von „Mittelerdischkeit“ abgesucht haben, so würden im Falle von 
Last Samurai, die Filmtouristen nach Anzeichen von „Japanischkeit“ in der neuseeländischen Landschaft suchen (20II: I5I).

Als Gründe dafür, dass sich die Touristen anders verhielten, nennt Leotta die mangelnde Bewerbung der locations durch Warner Bros (ganz im Gegensatz zu Lord of the Rings), der Produktionsgesellschaft hinter Last Samurai, den Abbau der meisten Filmsets und ein selbstbehinderndes Verhalten der neuseeländischen Tourismusunternehmen aus Angst vor touristischer „Überflutung“ der locations (20II: 154). Als weiterer Grund kann hier wie bereits erwähnt mangelndes Hintergrundwissen über die genauen locations von Last Samurai angeführt werden, wodurch eine Assoziation mit Neuseeland gar nicht erst zustande kommt.

Um diesen Missstand zu beheben, scheinen viele neuseeländische Rezensenten die nationale Agenda Neuseelands, seine locations sowohl für Filmproduzenten als auch für Filmtouristen auf allen möglichen Distributionskanälen zu vermarkten, verinnerlicht zu haben. So liest sich die ausgesprochen positive Rezension von „(poorlittlerichboy@hotmail.com)“ fast wie eine Schleichwerbung für Neuseeland im Rahmen eines viralen Marketings:

Yet another film to be shot in New zealand with wonderful production values! We're doing quite well, aren't we? Shot at Mt Taranaki, the film's cinematography is nothing short of stunning and the film is very easy on the eyes. The special effects were terrific...in fact; did they even use special effects? It all looked so damn real to me (I5. Januar 2004). Neben dem expliziten Lob für die „stunning cinematography“ lässt sich in der rhetorischen Frage „We're doing quite well, aren't we?"“ auch ein seit dem Erfolg von Lord of the Rings erwachsener Nationalstolz neuseeländischer Zuschauer erblicken.

Insgesamt hat bei Last Samurai nicht nur die filmgeografische Inkongruenz zwischen Neuseeland und Japan, sondern auch die Tatsache, dass kurz zuvor Lord of Rings erschien und deshalb Mittelerde stark mit Neuseeland assoziiert wurde, zu Authentizitätsproblemen in den Augen nicht aller, aber vieler Zuschauer geführt. Außerdem lässt sich an den Rezensionen neuseeländischer Zuschauer eine deutlich erstarkte nationale „Landschaftsbewerbungsagenda“ feststellen, wie dies bei Filmen wie Vertical Limit, die vor Lord of the Rings und damit auch vor nation branding-Kampagnen wie „IO०\% Pure“ erschienen, nur in weitaus geringerem Maße festzustellen war. 


\subsubsection{Without a Paddle (2004)}

Der nur ein Jahr nach Last Samurai erschienene Film Without a Paddle war der erste Film, der von dem Large Budget Screen Production Grant, von der neuseeländischen Regierung finanziert, profitierte. Ebenso wie Last Samurai und Vertical Limit ist auch Without a Paddle, der von einer abenteuerlichen Reise dreier Freunde auf einem wilden Fluss in Oregon, USA, handelt, von einem transnationalen Produktionshintergrund geprägt. Der Entscheidung der amerikanischen Produzenten, in Neuseeland zu drehen, liegen deshalb ebenfalls primär finanzielle Gründe zugrunde: dem Rückerhalt von I2,5\% des Produktionsbudgets Dank des Large Budget Grants. ${ }^{54}$ Im Gegensatz zu Last Samurai und Vertical Limit, die primär von ausländischen Produzenten finanziert wurden, wurde insofern die Delokalisierung und gewissermaßen auch in symbolischen Sinne die Denationalisierung der neuseeländischen Landschaft hier in stärkerem Maße von Neuseeland selbst vorangetrieben - ein Umstand, der bereits von vielen Neuseeländern kritisiert worden ist.

Im Gegensatz zu den Filmemachern von Last Samurai oder Vertical Limit versucht Regisseur Steven Brill bei der Diskussion seines Films Without a Paddle gar nicht erst, ihn als creative runaway zu vermarkten, sondern stellt klar, dass vor allem pragmatische und ökonomische Gründe den Film zu einer economic runaway nach Neuseeland gemacht haben:

The weather (...) you know, was the opposite. Like it was fall here [in den USA] and we shot there and it was summer. It was cheaper. And we could use Peter Jacksons crew. Which had just finished Lord of the Rings. It was a great way to be away (...) from Hollywood (Brill, o. J.: 02:17-02:35 min.).

Dadurch entsteht der Eindruck, dass der Dreh in Neuseeland eine Verlegenheitslösung darstellte, vor allem, weil die Notwendigkeit, den Dreh auf als heilig erachtetem Land durch Māori absegnen zu lassen, als Produktionshindernis betrachtet wurde („We also had to get Māori blessing to shoot on their land. While they were very accommodating, you can imagine how this can delay scenes“ [Brill, Without a Paddle, 2004]).

Zwar betont Brill, dass er anfangs aus Authentizitätsgründen skeptisch gewesen sei, den Film in Neuseeland zu drehen, er aber dann zu der Überzeugung gelangt sei, dass Neuseeland ein „perfektes Double“ für Oregon darstelle (Brill, The Steven Brill Interview, 2004). Allerdings stellt der Regisseur seine eigene geografische Kompetenz infrage, wenn er im Audiokommentar auf der DVD freimütig zugibt, noch nie in Oregon gewesen zu sein. Insofern klingt seine Aussage „I've

\footnotetext{
${ }^{54}$ www.imdb.com/title/tto36475I/trivia?ref_=tt_trv_trv
} 
never been to Oregon. [...] Yet I believe they're a pretty good match" (Without a Paddle, Audiokommentar [00:16:19-0o:16:29]) wenig überzeugend und kann eigentlich kaum als ernsthafte „Authentisierungsstrategie" eingestuft werden.

Während also in paratextueller Hinsicht die filmgeografische Authentizität des Films für den Zuschauer eher untergraben wird, werden im Filmtext selbst durchaus Bemühungen angestellt, die neuseeländischen Whanganui-, Rotorua-, und Taupo-Regionen in ein überzeugendes Oregon-Setting zu verwandeln. Neben dem Einsatz eines stock shots, d. h. einer vorgefertigten Filmszene, die tatsächlich in Pennsylvania gedreht wurde (Audiokommentar, Without a Paddle [oo:07:20]), wurden auch ein US-Verkehrsschild mit der Aufschrift „Portland I94 MI“ (Without a Paddle [oo:I6:Io]) sowie US-Flaggen in der neuseeländischen Kleinstadt Raetihi platziert ([oo:I7:19]), um dem Zuschauer das Oregon-Setting vorzugaukeln. Die folgende Abbildung soll den konkreten Umwandlungsprozess der neuseeländischen Kleinstadt Raetihi in eine namenlose Kleinstadt in Oregon verdeutlichen:

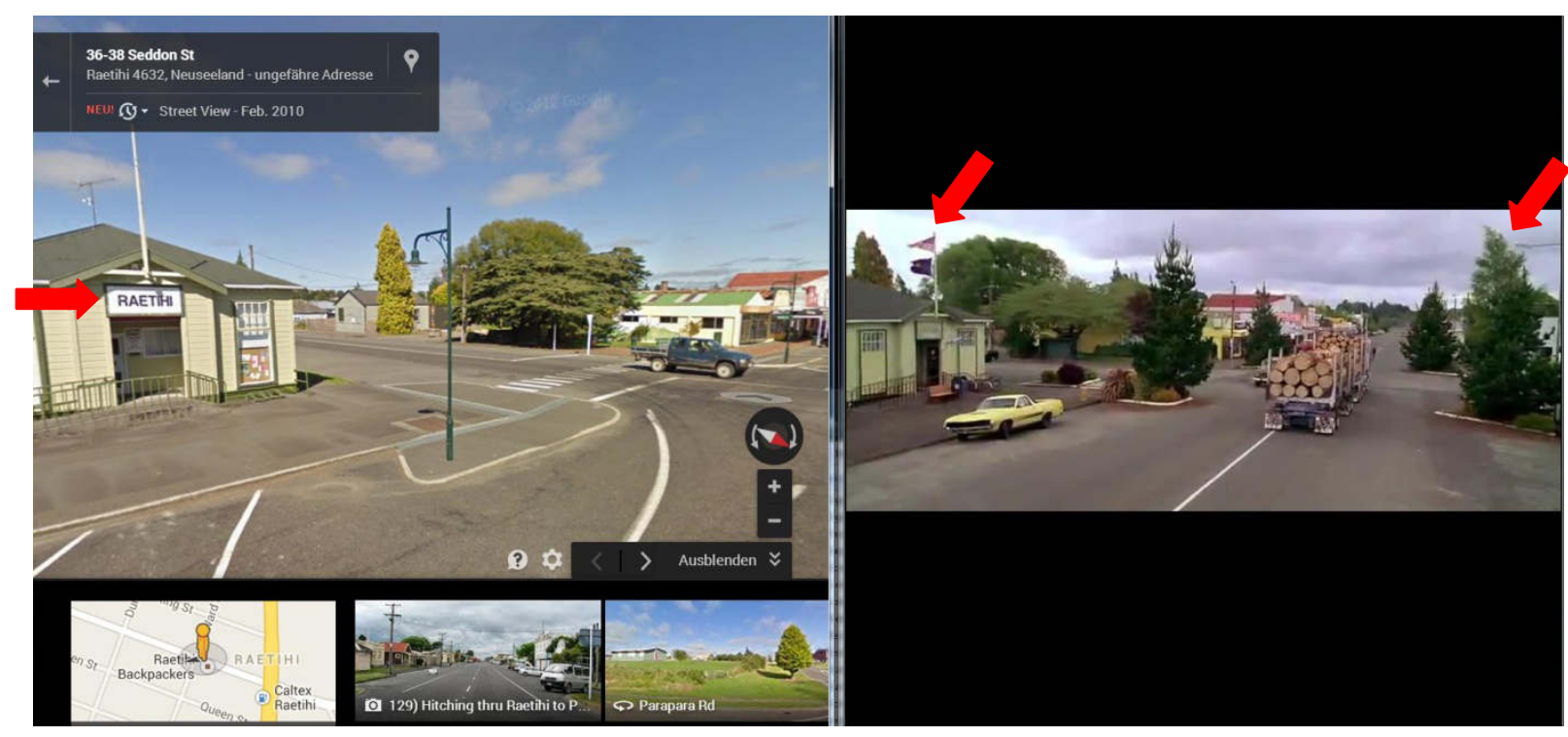

Abbildung 3.Io: Google Streetview-Ausschnitt aus Raetihi [links, „Raetihi“], Filmszene aus Without a Paddle [0o:17:19]

Durch den Vergleich der Filmszene (rechtes Bild), in der die drei Protagonisten einen Zwischenstopp in einer namenlosen Kleinstadt in Oregon machen (Without a Paddle [oo:17:19]), mit einer Google Streetview-Aufnahme von Februar 20 or (linkes Bild, „Raetihi“), lässt sich der semiotische Entleerungs- und Befüllungsprozess im Rahmen der filmgeografischen Delokalisierung anschau- 
lich nachvollziehen: So ist zu erkennen, dass es sich bei der Aufschrift „RAETIHI“ auf einem lokalen Gebäude (linkes Bild) um einen neuseelandspezifischen Marker handelt, der in der Filmszene entfernt wurde (Entleerung), um dann die nunmehr (fast) nationsunspezifische Projektionsfläche mit dem US-spezifischen Marker einer auf demselben Gebäude platzierten US-Flagge (rechtes Bild, Befüllung) zu „amerikanisieren“. Des Weiteren wurden in Neuseeland nicht-endemische, als Oregon-spezifisch erachtete Nadelbäume platziert.

Eine weitere Authentisierungsstrategie, die die Filmemacher einsetzten, war das Filmen in nicht-endemischen neuseeländischen Mammutbaum/Rotholz-Wäldern, die eigentlich „US-typisch“ sind: „Redwood forest is not indigenous to New Zealand. In fact, no trees are indigenous to New Zealand." 55 (Without a Paddle, Audiokommentar [00:52:45-00:52:53]). Diese Strategie stellt einen Rekurs auf das koloniale Erbe Neuseelands dar: Die Sehnsucht der europäischen Kolonialisten, die als befremdlich empfundene neuseeländische Flora und Fauna durch importierte Flora und Fauna „anzureichern“ und „aufzuwerten“ und dadurch der verlassenen Heimat anzugleichen (Phillips, 2012), stellt heutzutage für Neuseeland einen Vorteil in seiner Bewerbung als location für Handlungsorte diverser nicht-neuseeländischer Handlungsorte dar. Denn gerade aufgrund der „Vorarbeit“ der Kolonialisten kann sich Neuseeland heute als landschaftlich äußerst vielfältig darstellen. Ein beträchtlicher Teil dieser Diversität ist somit menschengeschaffen und keineswegs naturgegeben.

Tatsächlich werten einige Zuschauer die Filmgeografie des Films als authentisch. „P. Ryan 'Oregon Girl'“' beschreibt die Wirkung des Film auf sie folgendermaßen: Having lived in Oregon for two years with my daughter we got quite a kick out of the movie. Even though the movie was filmed in New Zealand it made you feel like you really were in Oregon. (I. Oktober 2005). Bemerkenswert ist hier, dass die beiden Zuschauer sowohl aus Oregon selbst stammen als auch das metatextuelle Hintergrundwissen besitzen, um zu wissen, dass der Film eigentlich in Neuseeland gedreht wurde und den Film dennoch als authentisch („,it made you feel like you really were in Oregon“) empfinden. Diese Rezeption lässt sich aber insofern als Sonderfall einstufen, als dass der nostalgische Wunsch, die frühere Heimat Oregon noch einmal in einem Film nacherleben zu können, dabei geholfen hat, die filmgeografische Inkongruenz zu ignorieren und die Authentisierungsstrategien der Filmemacher sehr bereitwillig zu akzeptieren.

\footnotetext{
" Die Aussage des Regisseurs, es gebe überhaupt keine endemischen Bäume in Neuseeland, ist inkorrekt. So sind Kauri- und TotaraBäume gute Beispiele für endemische neuseeländische Bäume, die nicht importiert worden sind.
} 
Trotz der vielfältigen Authentisierungsstrategien ist Without a Paddle von vielen Zuschauern, insbesondere von weniger nostalgischen Zuschauern aus Oregon, als filmgeografisch inauthentisch eingestuft worden. So hat der Rezensent „will 7370 “ auf der IMDb einen ganzen Thread ${ }^{56}$ eröffnet, in dem ausschließlich über die geografischen Inkongruenzen des Films diskutiert wird. Dabei bemängelt der User, dass der Einsatz neuseeländischer locations zur Simulation eines filmischen Oregons andere Zuschauer, die Oregon nicht kennen, in deren geografischen Erwartungen an Oregon fehlleiten würden. Neben den ikonischen „palm trees“, die schon bei Last Samurai zu Irritationen bei vielen Zuschauern geführt hatten und auch von der IMDb als goof in der Kategorie „Errors in Geography“ eingestuft wurden, sieht der Rezensent die neuseeländischen „clear, blue rivers“ als Fehlrepräsentation der Oregon-Landschaft an. Er bringt keinerlei Verständnis für die filmgeografische Inkongruenz auf und schätzt die Produktionsbedingungen falsch ein, wenn er vermutet, dass ein Dreh in Oregon günstiger gewesen wäre als in Neuseeland. Für den Rezensenten wäre nur ein Dreh in Oregon akzeptabel gewesen, einen Dreh in Neuseeland wertet er in puncto Authentizität als unverzeihlichen „Vertragsbruch“ der Filmemacher und „disservice“ gegenüber Oregon (20. Juni 2008 09:I0:35). An dem Kommentar („giving people the wrong idea“) wird auch klar ersichtlich, dass Zuschauer auch heute in Zeiten der Globalisierung, wo Filmdrehs immer häufiger an locations gedreht werden, die mit dem Handlungsort nicht übereinstimmen, dennoch Filme als eine Informationsquelle über die Geografie fremder Länder behandeln. Der User spekuliert gar, dass Zuschauer aufgrund des Films aus den „falschen Gründen“ motiviert sein könnten, nach Oregon zu ziehen: „I'd just hate to have someone watch the movie and say, [...] 'Cool, let's sell the place and move to Oregon where we can buy a house on the banks of one of those pure blue rivers'“ (26. Juli 2008 I4:44:2I).

Dass Without a Paddle tatsächlich zu einer verzerrten Imagination Oregons führen kann, illustrieren Rezensionen wie die von „Hunky Stud“: „At first, I was really impressed by the natural beauty of Oregon, but it was actually shot in New Zealand. The blue river, the green mountain, the fresh air, I wish that I can live in a place like that" (I8. Juli 2008). Während der Rezensent zuerst fälschlicherweise annahm, dass der Film „wirklich“ die Schönheit Oregons zurschaustelle, konnte er allerdings seine geografisch verzerrte Imagination durch meta- oder paratextuelle Informationen nachträglich noch korrigieren, was aber nicht bei jedem Zuschauer der Fall sein wird.

\footnotetext{
${ }^{56}$ Ein „Thread“ ist eine hierarchisch organisierte Diskussion in einem Diskussionsforum.
} 
Das Wissen um die Inauthentizität des Films kann sogar zu einer Steigerung des filmischen Genusses führen, wie die Rezension von „Pookyiscute“ aus Ungarn zeigt: „It was awesome actually being in Oregon and seeing this movie which takes place in Oregon (even though it was filmed in New Zealand), and each time they would mention something about the state or show footage of something that looked like it, the audience responded gleefully“ (I5. Februar 2005). Dass der Zuschauer sich hierbei tatsächlich während des Betrachtens des Films in Oregon befand und gerade die filmgeografische Inkongruenz die Zuschauer zu einer imagined community zusammengeschweißt hat, hat hierbei zu einem fast surrealen Erlebnis geführt.

Ähnlich wie schon bei Last Samurai versuchen auch hier neuseeländische Rezensenten „jimbo_4I35“, die von anderen Zuschauern bemängelte „Inauthentizität“ des Films relativieren und verfolgen eine offensichtliche nationale clean ES green-Image-Agenda (27. Oktober 2008 2I:5O:II). Nicht nur soll die ökologische Reinheit Neuseelands beworben, sondern zudem suggeriert werden, dass Oregon von Umweltverschmutzung geplagt sei, Neuseeland hingegen nicht. Das Eigenlob vieler neuseeländischer Zuschauer über „ihr“ Land ist teilweise derart offensichtlich, dass dies explizites Missfallen über die Popularität Neuseelands als location bei nicht-neuseeländischen Zuschauern ausgelöst hat. So schreibt „JackBluegrass“: „A country that's stealing movie making from all other nations, because of all the talent there“ (30. März 20I4 09:36:36). Aus dieser Äußerung ist zugleich Missfallen aber auch Anerkennung des „Talents“ Neuseelands, seine Landschaft und die Arbeitskräfte erfolgreich zu vermarkten, ersichtlich. Dass sich Neuseeland in der globalen Sphäre als location-Vermarkter in einem Konkurrenzverhältnis zu anderen Ländern befindet und sich eine Vormachtstellung erarbeiten konnte, wird somit von dem Rezensenten zwar anerkannt, aber letztendlich negativ („stealing“) bewertet. Dass diese Konkurrenz zwischen Ländern, die sich als location für transnationale Produktionen anbieten wollen, auch filmtouristische Folgen hat, illustriert der als nächstes zu analysierende Film The Waterhorse: Legend of the Deep.

\subsubsection{The Waterhorse: Legend of the Deep (2007)}

Bei dem Film The Waterhorse: Legend of the Deep handelt es sich um eine vorwiegend ausländische Ko-Produktion, die nicht nur bezüglich des Handlungsortes und der locations, sondern auch in narrativischer Hinsicht deutliche Parallelen zum 2005 erschienenen Film Mee-Shee - The 
Water Giant aufweist. ${ }^{57}$ The Waterhorse: Legend of the Deep erzählt die Geschichte von Angus, einem Jungen, der am Loch Ness in Schottland zur Zeit des Zweiten Weltkriegs aufwächst, sich ebenso wie Protagonist Mac in Mee-Shee mit einer Wasserkreatur anfreundet und sich für ihre Rettung einsetzt. Zwar ist die Legende vom Loch Ness-Monster eine spezifisch schottische Legende, Angus' Reiten und Abtauchen auf dem Wasserdrachen und die Flucht des Wasserdrachen ins Meer kann aber als klare intertextuelle Referenz zum Film Whale Rider gesehen werden: Auch Paikea reitet auf dem Rücken eines Wassertieres und taucht zeitweise in die Tiefe hinab, um schließlich die Wale ins Meer und damit in die Freiheit zu führen. ${ }^{58}$

Um den schottischen Handlungsort des Films zu authentisieren, sind Strategien eingesetzt worden, wie sie bereits bei den vorigen Filmen besprochen worden sind. So wurden ähnlich wie bei Without a Paddle auch hier stock shots mit den schottlandspezifischen Schlössern bzw. Ruinen Eilan Donan und Urquhart eingesetzt und im Making Of ähnlich wie bei Vertical Limit die „objektive“ Ähnlichkeit zwischen neuseeländischer location und ausländischem Handlungsort betont. So kommentiert der Regisseur Jay Russell im Making Of:

At the end of the day [...] it was a matter of having practicality meet creatively what you $[\ldots]$ want to achieve and also to meet authenticity which is $[\ldots]$ important to me. So we managed to find this nice blend [...] of Scotland itself and New Zealand, which, as it turns out, could be sister countries, because they do look alot alike (The Waterhorse: Legend of the Deep, „Featurettes: Setting the Scene“ [oo:or:3800:02:03]).

Auch der punktuelle Dreh an Originalschauplätzen in Schottland stellt wie bei Last Samurai den Versuch dar, dem Handlungsort die nötige historische Tiefe und Aura zu verleihen:

\footnotetext{
${ }^{57}$ Aufgrund sehr ähnlichen Rezeption von Mee-Shee, der ebenfalls am Whakatipu-See gefilmt wurde und von der Begegnung des Jungen Mac mit einer an die Ogopogo-Sage der lokalen Ureinwohner angelehnten Kreatur handelt, soll hier nur The Waterhorse: Legend of the Deep analysiert werden, insbesondere, weil The Waterhorse: Legend of the Deep aufgrund des Making Ofs eine tiefergehende Analyse erlaubt als Mee-Shee, zu dem es kein Making Of gibt. Festzuhalten ist hier allerdings, dass das Abfilmen des Milford Sound - einer der ikonischsten und touristisch bekanntesten Landschaften in Neuseeland - in Mee-Shee die Bemühungen der Filmemacher, ein ansonsten ,authentisches“ British Columbia-Setting zu vermitteln, ad absurdum führt, da der Milford Sound aufgrund seiner Bekanntheit kaum für British Columbia gehalten werden wird und somit auch kaum ein Zuschauer geneigt sein dürfte, die Darstellung von British Columbia im Film als „repräsentativ“ für das reale British Columbia anzusehen.

${ }^{58}$ Whale Rider, Mee-Shee und The Waterhorse: Legend of the Deep illustrieren durch ihre intertextuellen Referenzen die Transkulturalität mythischer Wasserkreaturen, die für die jeweiligen Kulturen eine identitätsstiftende Wirkung haben und geografischen Regionen (See/Ozean) eine magische Aura verleihen. Diese Mythen werden von den „weißen “ Regisseuren oben genannter Filme aufgegriffen und mit der europäischen Vorstellung einer romantischen Kind-Natur-Beziehung verknüpft. Diese Umwandlung und „Verniedlichung“ der ursprünglichen Mythen im Rahmen der Produktion generischer Familienfilme, die auf ein möglichst großes globales Publikum zugeschnitten sind und vor allem durch den hierfür grundlegenden Film Free Willy (1993) populär geworden sind, stellt eine kommerzielle Verwertung dar, die im Falle von Whale Rider von einigen Māori kritisiert worden ist (z. B. Hokowhitu, 2007). Anders verhält es sich bei The Waterhorse: Legend of the Deep, wo es weder während des Filmdrehs noch später in den Rezensionen Beschwerden über eine „Entweihung“ oder „Verkitschung“ der Loch Ness-Legende gegeben hat, sondern die erneute Popularisierung der Legende vielmehr positiv eingestuft worden ist, was auch viele Rezensionen belegen.
} 
„It was always very important to me to shoot here [in Schottland] because it is a story about Scotland and it is a story about a Scottish legend and I mean Scotland is ancient and I think all the mystery and [...] the legend of this piece was born on this ground" (The Waterhorse: Legend of the Deep, „Featurettes: Setting the Scene“ [0o:13:12-0o:13:24]). ${ }^{59}$ Das, was den Filmemachern in Neuseeland fehlte, war die historische Tiefe der schottischen Landschaft mit ihren alten Mauern und Gebäuden, die sie nur in Schottland selbst fanden: „The only thing missing is that in Scotland, there are old walls and buildings everywhere, so it was valuable to actually go to Scotland to get the representation of the age of the landscape“ (Miller, 2007).

Diese Strategien konnten viele Zuschauer dennoch nicht überzeugen, insbesondere, weil die Form und die Umgebung des Whakatipu-Sees deutlich erkennbar von derjenigen des Loch Ness abweicht. Auch hier sind es vor allem diejenigen Zuschauer, die in Schottland leben oder es als Touristen besucht haben, denen die filmgeografische Inkongruenz unmittelbar ins Auge sticht: „I spent about 2 hours around Loch Ness 2 years ago. When I first saw the trailer for this movie last summer. I told my brother, I don't know where this movie was filmed at, but that is NOT Loch Ness!“( „amleeparker“, 2I. Januar 2008 09:15:37). Zwar kann die Rezensentin die location aufgrund fehlenden metatextuellen Hintergrundwissens nicht positiv als Neuseeland idenfizieren, sie kann aber aufgrund ihrer detaillierten Kenntnisse über die Geografie von Loch Ness in negativer Hinsicht erkennen, dass die location nicht Schottland gewesen sein kann. User „godzillageek“ informiert mit seinem Post andere Zuschauer detailliert über die Eigenschaften des realen Loch Ness:

In the movie Loch Ness is actually right up against the ocean! In real life, Loch Ness is a freshwater lake and is connected to the ocean by the I2 kilometer-long River Ness. The River Ness is pretty wide, but very shallow in places, and nothing the size of a dinosaur could swim all the way through to the ocean (5. Januar 2008 09:05:15).

Für den Rezensenten „villard“ macht aber gerade die Tatsache, dass der reale Loch Ness nicht direkt in den Ozean mündet, dessen mystische Aura aus, die er im Film demnach zerstört sieht: „Loch Ness does not directly link to the ocean. This is exactly the mystique about Loch Ness: it is the isolated home of an isolated creature“ (20. April 2008). ${ }^{60}$ Die Enttäuschung über diese filmgeo-

\footnotetext{
${ }^{59}$ Die Encyclopaedia Britannica informiert darüber, dass auch der Whakatipu-See nach einer Māori-Sage ein Wassermonster enthält: „The lake's name is of Māori derivation and may mean 'water springs dug by Rakaihaitu' or, more likely, may refer to a legend of a taniwha (demon) living below the surface whose breathing was believed to cause the unusual 5 -inch (12s-mm) rise and fall in the lake's water level” („Whakatipu Lake“). Diese Parallele zur Loch Ness-Legende dürfte bei der Motivation der Filmemacher, am Whakatipu-See zu drehen, allerdings keinerlei Rolle gespielt haben.

${ }^{60}$ Bemerkenswert ist hier, dass die geografische Charakteristik des Whakatipu-Sees den Filmplot beeinflusst zu haben scheint: Während es im Making Of keine direkten Anhaltspunkte dazu gibt, so scheint dennoch die Nähe des Whakatipu-Sees zum Meer die Filmemacher dazu bewegt zu haben, ähnlich wie bei Free Willy das Ausbrechen des Wasserpferdes ins Freiheit symbolisierende
} 
grafische Inauthentizität ist in vielen anderen Rezensionen deutlich zu vernehmen, wobei „markstuart-I“ deshalb zu dem Urteil kommt, dass der Dreh in Neuseeland der „biggest goof“ des ganzen Films sei und quasi einen „Vertragsbruch“ seitens der Filmemacher darstellt („filming it in new zealand was a mistake“, 4. Juli 2008 I6:52:58). „Williambulmer“ fragt schließlich, ob das reale Schottland nicht mehr authentisch genug sei, um als filmisches Simulakrum seiner selbst fungieren zu können: „Why is it not filmed in Scotland. Was Scotland not Scottish looking enough?“ (II. Januar 2008 07:26:35).

Die Enttäuschung hat dazu geführt, dass einige Zuschauer die neuseeländische Landschaft nicht nur als inauthentische Kopie, sondern auch als ästhetisch dem schottischen „Original“ unterlegen ansehen: „I found it a bit disappointing that they didn't use what they had- personally, the real Loch Ness and surrounding areas are more beautiful to me than the 'fake' loch ness depicted in the film.“ („Jmaren5“, 2I. Januar 2008 07:49:19). Unter dem IMDb-Thread „Not filmed in Scotland ${ }^{\text {“6I }}$ prallen regelrecht zwei konkurrierende nationale Agenden aufeinander, die in folgendem Dialog münden:

I think they chose NZ for one obvious reason - its scenery is breathtaking, and although Scotland is nice, it's nowhere close to the majesty of NZ wilderness. The movie wouldn't look so dramatic if it was shot in Scotland, that's it („contact-282“, I5. April 2008 2I:II:40).

What?? Scotland has amazing scenery. Contact, have you been to Scotland? And if so, where have you been? 'Nowhere close to the majesty of NZ'??? How do you figure? Scotland has some of the most beautiful natural landscapes I've ever seen. I know several Kiwis who would agree with that („JMarens“, 26. November 2008 07:00:23).

Während „contact-282“ kreative Gründe für den Dreh in Neuseeland unterstellt, um die schottische Landschaft durch eine als wesentlich sublimer erachtete neuseeländische Landschaft „aufzuwerten" und zu einem für den Zuschauer noch beeindruckenderen visuellen Spektakel zu machen, stellt „Jmaren ““ in einer emotionalen Antwort die geografische Kompetenz des vorgenannten Re-

Meer als filmisches Finale einzusetzen. Womöglich hätte ein Dreh am originalen Loch Ness aufgrund seiner isolierten Position ein anderes Filmende erfordert.

${ }^{61}$ www.imdb.com/title/tto760329/board/nest/94499719?p=I (Zugriff: 28. März 2015) 
zensenten und dessen Argumentierung infrage. Unter den Usern ist also eine Konkurrenz ersichtlich, bei der darum gewetteifert wird, welche Landschaft die ästhetisch höherwertige sei.

Die von Rezensenten bereitgestellten metatextuellen Informationen führen wiederum bei anderen Rezensenten, die von einer authentischen Vermittlung des Loch Ness ausgegangen waren, zu offensichtlicher Enttäuschung. So zeigt sich „Irishbankslave“ darüber enttäuscht, dass er aufgrund des Films letztendlich eine verzerrte Vorstellung von Schottland hatte: „That's disappointing. I love this film, it made me fall in love with Scotland and now I find out it was actually filmed in NZ. Ah well, I'm sure Scotland is equally as beautiful and I would still like to visit there someday“ (2. Dezember 2012 07:00:42). An dieser Rezension wird wie schon bei Without a Paddle ersichtlich, dass Zuschauer auch heute noch Filme als Informationsquellen über die Geografie fremder Länder nutzen und als eine Form des virtuellen Tourismus betrachten, um das Ziel bereits vorab erleben zu können. Trotz der gängigen Praxis, gerade in Zeiten der intensivierten Globalisierung, Filme an locations zu filmen, die geografisch nicht mit dem Handlungsort übereinstimmen, scheint sich ein Lerneffekt bei den Zuschauern und eine gesteigerte Medienkompetenz nur zögerlich durchzusetzen. Zwar gibt es, wie in den vorigen Analysen angeführt wurde, durchaus Zuschauer, die eine medienreflexive Rezeptionsweise erkennen lassen, die Anzahl an Zuschauern, die sich von der geografischen Inkongruenz vieler Filme überrascht zeigt und kein Verständnis für die pragmatischen Produktionshintergründe zu haben scheint, ist aber weiterhin überraschend hoch.

Die Verdrängung schottischer durch neuseeländische locations auf der Mikroebene des Films The Waterhorse: Legend of the Deep korreliert mit der generellen Konkurrenz zwischen Schottland und Neuseeland auf der globalen Makroebene, um durch möglichst ausgefeilte nation branding-Strategien insbesondere hochbudgetierte Blockbuster-Filme und Touristen anzulocken. So wirbt auch Schottland mit seiner landschaftlichen Diversität, der ausgebauten Infrastruktur, „unberührter Wildnis“ und erfolgreichen Filmen wie Braveheart und Harry Potter. ${ }^{62}$ Letztendlich hat The Waterhorse: Legend of the Deep trotz der Bewerbung der locations sowohl auf schottischen $^{63}$ als auch auf neuseeländischen ${ }^{64}$ Tourismusseiten nur in Schottland signifikanten Filmtourismus ausgelöst, insbesondere bei Amerikanern („NZ lake“, 20o8), was Tooke und Bakers These vom Filmtourismus zum vom filmischen Handlungsort repräsentierten Land bestätigt:

\footnotetext{
${ }^{62}$ z. B. www.scotland.org/creative-scotland/film; Harry Potter kann in vielerlei Hinsicht als schottisches Pendant zu Lord of the Rings betrachtet werden: Wenn auch „Hogwarts “ in Harry Potter noch in Schottland positioniert ist, so handelt es sich doch um eine Parallelwelt, die wie „Mittelerde“ diverse Fantasyelemente aufweist und filmisch ebenso CGI-lastig umgesetzt worden ist. Wie auch bei Lord of the Rings zeigt sich, dass das Schottland in Harry Potter von Folge zu Folge immer stärker digitalisiert wird.

${ }^{63}$ z. B. www.lochness.com/waterhorse.htm (Zugriff: 13. August 20I4)

${ }^{64}$ z. B. www.skydiveparadise.co.nz/glenorchy.html (Zugriff: I3. August 20I4)
} 
It seems that if the film location is the true setting, the visitor visits the location: if the film location represents a fictional setting, the visitors go to the location; but if the film location represents a different actual setting, the visitors go to the place represented (Tooke and Baker, 1996: 93).

Diese Tendenz der Zuschauer, das vom Handlungsort repräsentierte Land (und nicht die location) zu besuchen, lässt sich unter anderem auf die große Anzahl uninformierter Zuschauer zurückführen, die The Waterhorse: Legend of the Deep irrtümlicherweise für einen Film der ersten Simulationsstufe halten: So schreibt „Amy Adler“ aus Toledo, Ohio: „[... the Scottish setting is glorious, making one want to hop on an airplane immediately for a visit, and the cinematography is most wonderful“"(16. Februar 2008). Bezeichnend ist, dass die Filmtouristen nach Schottland, also an den im Film repräsentierten Ort, gereist sind - und nicht zur neuseeländischen location, der dieser filmischen Repräsentation größtenteils zugrunde liegt. Trotz einiger Rezensionen, die Neuseeland als authentisches Double für Schottland betrachten (z. B. „Geoff O'Callaghan“ [4. Mai 20o8]) hat der Film somit primär ein touristisches Interesse an Schottland ausgelöst; das reale Schottland ist diesen Filmtouristen also wichtiger als ein zwar ebenso reales Neuseeland, dass aber im filmischen Kontext nicht als hyperreales, sondern als ein „falsches“ Schottland angesehen wird. Obwohl somit sowohl Schottland als auch Neuseeland in semiotischer Hinsicht als Referenten fungieren (vgl. Abbildung 3.4), wird der dem filmischen Handlungsort zuzuordnende Referent „Schottland“ als wesentlich stärker eingestuft.

\subsubsection{Good for Notbing (2011)}

Good for Notbing ist der vierten Simulationsstufe zuzuordnen und ist der einzige der hier analysierten Filme, bei dem es sich um eine rein lokale Produktion handelt und die somit auch keine runaway-Produktion darstellt. Die Tatsache, dass neuseeländische Filmemacher neuseeländische locations einsetzen, um nicht etwa ihre eigenen nationalen Geschichten zu erzählen, sondern die anderer Länder, stellt einen neuen Trend dar, der in dieser Form bisher nur selten zu beobachten war. Diese Abwendung von nationalen Thematiken kann als eine Form der willed dislocation be- 
schrieben werden, einem von Janet Wilson bei ihrer Besprechung von Elizabeth Knox ${ }^{65}$ Roman The Vintner's Luck verwendeten Begriff. Wilson schreibt:

$\left[\right.$ Knox $\left.^{6}\right]$ story is set in a remote (from New Zealand) region (Burgundy in France) and a distant historical era (just after the Napoleonic Wars). This form of willed dislocation from her country of origin can be interpreted in terms of a controlled re-inscription of the original trauma of the New Zealand settler, catalysed by their distance and dislocation from the homeland; the regional, provincial focus of her story continues the interest in the ethos and teleology of localization which her autobiography suggests (Wilson, 2004: I24).

Wilson betrachtet Knox' Entscheidung, einen Roman mit ausländischem Handlungsort zu schreiben, als eine bewusste, eine willed dislocation, um dadurch das ursprüngliche Trauma der neuseeländischen Kolonialisten auf symbolischer Ebene zu reimaginieren, indem sie sich als Schriftstellerin ebenfalls auf eine gedankliche Reise zu einem ihr geografisch und zeitlich weit entfernten, fremden Ort (historisches Frankreich) begibt. ${ }^{66}$

Auch die Entscheidung von Regisseur Mike Wallis, sich in einen geografisch und zeitlich fernen Handlungsort, den amerikanischen Wilden Westen, hineinzuversetzen, kann als willed dislocation betrachtet werden. Gleichzeitig ist hierin der Wunsch erkennbar, die neuseeländischen locations durch Delokalisierung zu denationalisieren, um sie dann mit der mythischen Aura des Wilden Westens aufzuladen. Denn auch wenn die Motivation, durch die Produktion eines generischen Westernfilms ein möglichst breites globales Publikum anzusprechen, nicht unerheblich gewesen ist, so lässt sich gleichzeitig das Bedürfnis der Filmemacher erkennen, durch „Amerikanisierung“ die neuseeländische Otago- und Mackenzie-Steppenlandschaft als mythischen Wilden Westen, einem Ort ihrer Sehnsucht, zu reimaginieren und „aufzuwerten“, wie folgendes Interview illustriert:

INTERVIEWER: What was the inspiration for making a Western movie in New Zealand? WALLIS: I was inspired by the Spaghetti Westerns, especially the ones done by Sergio Leone. I love the fact that they made them in Italy and Spain but they were set in America

\footnotetext{
${ }^{65}$ Elizabeth Fiona Knox ist eine 1959 in Wellington geborene Schriftstellerin, deren bekanntester Roman The Vintner's Luck 1998 publiziert wurde.

${ }^{66}$ Knox' Roman wurde 2009 von Niki Caro (der Regisseurin von Whale Rider) verfilmt und kann neben Good for Nothing zumindest in Bezug auf die Regisseurin als weiteres Beispiel einer willed dislocation angefuhrt werden. Allerdings handelt es sich bei The Vintner's Luck um eine transnationale Produktion, an der im Gegensatz zu Good for Nothing auch ausländische Produzenten, darunter Frankreich und Belgien, mitgewirkt haben. Vor allem aber wurde der Film primär in Frankreich gedreht, und nur kurze Szenen in Belgien und Neuseeland, sodass der Film der 2. Simulationsstufe zuzuordnen ist und deshalb im Rahmen dieser Analyse nicht behandelt wird.
} 
and they have become iconic Westerns in their own right. And I thought if the Italians could do it, then surely the Kiwis can too!

INTERVIEWER: With such vibrant landscapes and untrammeled ground, New Zealand seems more like the Wild West than the overdeveloped West does now. Are you surprised that there are not more Westerns being done?

WALLIS: I am surprised we are first ones to do it ${ }^{67}$ - and that it's taken this long for one to be made. I've been dreaming about making one since I started going there as a little kid. I've always felt a connection between the landscapes of Central Otago in New Zealand and the Italian/Spanish landscapes and the landscapes I was seeing from the American West on the screen. I think there's a lot of scope for doing more Westerns in New Zealand (Wallis, 200I).

So wie italienische und spanische locations durch ihren Einsatz als geografisches „Double“ für den Wilden Westen selbst ikonisch geworden sind, so erhofft sich also auch Wallis, dass er die neuseeländischen locations in dieser Weise zu ikonischem Status verhelfen kann.

Ähnlich wie es schon Zwick bei Last Samurai tat, bedient sich auch Wallis einer kompensatorischen Strategie, wenn er argumentiert, dass die neuseeländischen locations aufgrund ihrer Unberührtheit historisch authentischere Orte für die Rekonstruktion des Wilden Westens seien als der eigentliche, aber durch Modernisierung seiner Aura des Vormodernen beraubte amerikanische Westen. Hiermit wird quasi suggeriert, dass der heutige amerikanische Südwesten seine Authentizität als location für einen historischen Handlungsort eingebüßt habe und dass das neuseeländische Otago - wie im Interview (s. o.) argumentiert wurde - ohnehin eine nach Ansicht des Filmemachers verblüffende Ähnlichkeit mit dem historischen Westen der USA aufweise, was wiederum eine implizite Projektion der eigenen Sichtweise auf die Zuschauer darstellt.

Aber auch auf nicht-landschaftlicher Ebene sieht Wallis Gemeinsamkeiten zwischen Neuseeland und den USA, die seiner Ansicht nach einen kiwi-Western zusätzlich legitimieren: „I think the culture of the 'Southern Man' in New Zealand is very similar to that of the Western Cowboy“ (Wallis, Directors Statement, o. J.). Hiermit unterstreicht der Regisseur Ähnlichkeiten in der kolonialen Historie der beiden Länder, die seiner Ansicht nach auch zwei ähnliche Männertypen her-

\footnotetext{
${ }^{67}$ Während es sich bei Good for Nothing um den ersten kiwi-Western handelt, so hat es doch in der Geschichte des zeitgenössischen neuseeländischen Kinos etliche Filme gegeben, die auf ästhetischer und narrativischer Ebene bestimmte Tropen des USWesterns bereits früher aufgegriffen haben, darunter Der Kampfkoloss als „Endzeitwestern“, aber auch Filme mit neuseeländischem Handlungsort wie Utu und Starlight Hotel, die respektive die New Zealand Wars und die Zeit der Großen Depression thematisieren.
} 
vorgebracht haben: den neuseeländischen Southern Man, der eng mit dem archetypischen neuseeländischen Man Alone verwandt ist, und dem amerikanischen lonesome cowboy. Insofern erscheint es Wallis adäquat, sowohl auf geografischer als auch auf narrativischer Ebene die USA durch Neuseeland doublen zu lassen.

Trotz der vielen beteuerten landschaftlichen Gemeinsamkeiten zwischen Otago und dem Wilden Westen musste auch bei Good for Nothing die neuseeländische location aktiv delokalisiert werden, um den Wilden Westen nach Ansicht der Filmemacher glaubhaft simulieren zu können. Während ein Großteil des Films on location gedreht wurde, wurde der Showdown in einem Canyon kurz vor Ende des Films in einem Filmstudio, also off location, gedreht.
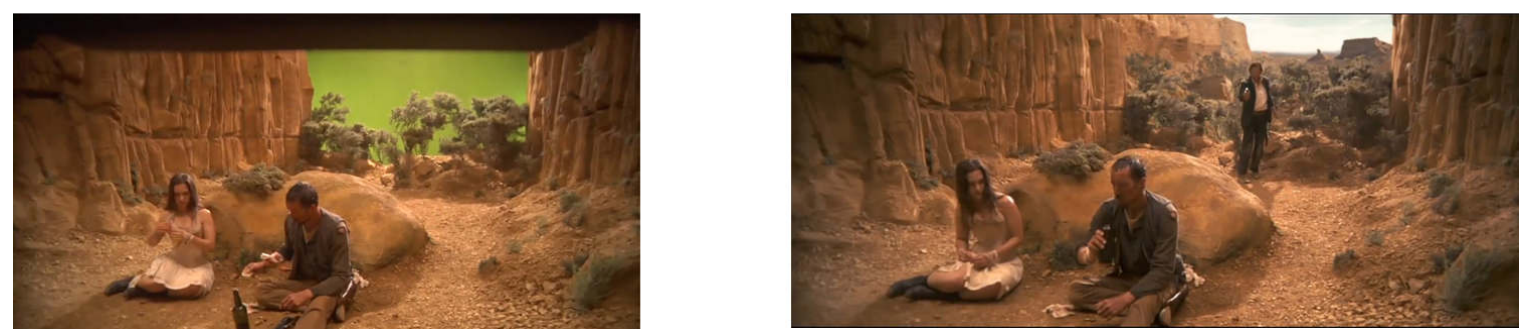

Abbildung 3.II: Showdown-Szene, links Studio-greenscreen, rechts in der Postproduction digital eingefügte neuseeländische location im Hintergrund [oo:Ir:44]

Das Making Of zeigt, dass bei der Darstellung US-typischer Canyons mit Kulissen nachgeholfen werden musste, da es in Neuseeland keine derartigen Canyons gibt (Abbildung 3.II). Hierbei wurden die Canyonwände mittels Polystyrol-Wänden in Fels-Optik nachgebildet und die neuseeländische Landschaft im Hintergrund per greenscreen-Verfahren hineinprojiziert. Dies illustriert, dass die neuseeländische und die amerikanische Geografie hinsichtlich des Vorhandenseins tief eingeschnittener Canyons eine klar erkennbare geografische Inkongruenz aufweisen, über die die Filmemacher aufgrund der Bedeutung von Canyons für generische Western nicht hinwegsehen konnten, sondern aktiv Canyons als US-typische Landschaftsmarker im Film platzieren mussten.

Für die Simulation einer typischen Wildwest-Stadt hingegen erwies es sich für die Filmemacher als Glücksfall, dass es in der Region tatsächlich einen Wildwest-Stadt-Nachbau namens Mellonsfolly als Touristenattraktion gibt. Da es sich bei der Stadt somit bereits um ein Simulakrum ähnlich wie Disneyland handelt, stellt die Filmstadt das Simulakrum eines Simulakrums, quasi eine doppelstufige Simulation, dar, was sich als wertvolle Hilfe für die Filmemacher erwies, die hier- 
durch die Produktionskosten senken konnten und deshalb einen größeren finanziellen Spielraum, insbesondere für den Nachbau des Film-Canyons und der digitalen Effekte, hatten.

Historisch betrachtet konnten die Filmemacher der in den I96oern und 7oern so populären Italowestern, die frühe Formen der Delokalisierung darstellten, nicht auf ausgeklügelte Techniken digitaler Rückprojektion zurückgreifen. Die hierbei eingesetzten italienischen und spanischen locations wiesen wie auch die hier analysierten neuseeländischen locations nur eine bedingte Ähnlichkeit mit dem amerikanischen Wilden Westen auf. Bei teureren Produktionen wie Sergio Leones Once Upon A Time In The West (1968) ${ }^{68}$ wurde aus diesem Grunde zwar hauptsächlich in Spanien (u. a. Almería) gedreht, aber auch auf stock shots des Monument Valley zurückgegriffen; es handelte sich hierbei also um ein Hybrid aus 2. und 4. Simulationsstufe, da zumindest kurze Passagen tatsächlich in den USA gedreht worden waren. Günstigere Produktionen wie die Django-Filmreihe mussten hingegen mit ausschließlich italienischen und/oder spanischen locations auskommen, um eigentlich urtypische amerikanische Landschaften zu imitieren. Zwar lassen sich im Internet zahlreiche Filmrezensionen heutiger Zuschauer zu Italowestern finden, logischerweise aber keine Rezensionen damaliger Zuschauer. Aus diesem Grund ist es schwierig, zu beurteilen, wie damalige Zuschauer die Handlungsorte dieser Filme bewerteten.

Aufgrund des immensen Erfolgs der Italowestern lässt sich aber mutmaßen, dass die Handlungsorte eher nicht als „,inauthentisch“ angesehen wurden. Dies lässt sich vor allem dadurch erklären, dass der Italowestern nur anfangs als „Kopie“ des klassischen Hollywoodwesterns betrachtet wurde, bald aber durch seine neuartige Ästhetik das Westerngenre an sich revolutionierte und sich vom Hollywoodwestern emanzipieren konnte. ${ }^{69}$ Statt der vergleichsweise „sterilen“ Ästhetik klassischer Hollywoodwestern setzte der Italowestern demgegenüber auf betont „schmutzige“, „harte“ Bilder und erzeugte dadurch einen besonderen Realismuseffekt. Durch den Erfolg der Italowestern könnte man sagen, dass die italienischen und spanischen locations selbst Teil der Wildwest-Ikonografie geworden sind und den imaginären Raum des Wilden Westens erweitert - aber keineswegs ersetzt - haben. Obwohl die spanischen und italienischen sowie andere europäische locations somit nicht als „de-authentisierende“ Störfaktoren in Westernfilmen erachtet wurden, ist Monument Valley zweifelsohne die populärste Attraktion für Westernfans, wohingegen beispielsweise die „Rancho Leone“ nahe der spanischen Stadt Almería trotz ihrer so wichtigen Rolle als location für

\footnotetext{
${ }^{68}$ Deutscher Titel: Spiel Mir Das Lied Vom Tod

${ }^{69}$ So hatten in der Folgezeit amerikanische Spätwestern (z. B. von Sam Peckinpah) wiederum die Ästhetik der Italowestern als Vorbild, um auch in den USA produzierte Western auf revisionistische Weise neu zu gestalten und sich von dem klassischen Hollywoodwestern abzuwenden.
} 
die Sweetwater-Farm in Once Upon A Time In The West weniger bekannt ist. Auch hier bestätigt sich demnach die These Tooke und Bakers, dass die filmtouristischen Ströme sehr häufig zum vom Handlungsort repräsentierten Land gerichtet sind, während die location weniger Aufmerksamkeit erfährt. Insofern lässt sich hier ein Kontinuum feststellen, da auch früher der (allerdings deutlich geringere) Filmtourismus primär zum vom Handlungsort repräsentierten Land führte.

Ein bedeutender Unterschied zu den bisher analysierten Filmen und auch zu den Italowestern ist die offensive Bewerbung der neuseeländischen locations, bei der man sich nicht auf die Filmcredits und Making Ofs beschränkt hat, sondern auch auf dem DVD-Cover („exquisitely filmed with stunning New Zealand as the stand-in for the sweeping Old West“ [Good for Notbing, DVD-Cover]) und der offiziellen Webseite direkt auf die neuseeländischen locations, die ja von Zuschauern eventuell als de-authentisierendes Element in einem Western gesehen werden könnten, hinweist. Dies steht in deutlichem Kontrast zu der Vermarktungsstrategie der zuvor analysierten Filme, bei denen weder auf den DVD-Covern noch auf den offiziellen Webseiten-Auftritten die neuseeländischen locations explizit beworben, sondern vielmehr „verschwiegen“ werden.

Dieser Unterschied lässt sich damit erklären, dass es neuseeländische Filmemacher ähnlich wie auch neuseeländische Zuschauer als wichtig empfinden, „ihre“ Landschaft einem globalen Publikum näherzubringen - und zwar unabhängig davon, ob auch der Handlungsort neuseeländisch ist oder nicht. Vielmehr hat Wallis sich auch deshalb für einen Western entschieden, weil gerade bei diesem Genre der Landschaft eine hohe Bedeutung beigemessen wird: „(...) one of the main ideas behind doing a western was that you get a lot of the value out of the landscape (...)“ (Good for Nothing, Making Of [oo:02:3I]). Während demnach der Dreh in Neuseeland für ausländische Filmemacher von Filmen wie beispielsweise Without a Paddle wie erörtert eine Verlegenheitslösung darstellte, sieht ein neuseeländischer Filmemacher wie Mike Wallis die neuseeländischen locations vielmehr als einen bedeutenden Pluspunkt seines Films, woraus sich auch ein gewisser Nationalstolz (insbesondere seit Lord of the Rings) speist. Interessanterweise harmoniert die offensive Bewerbung der neuseeländischen Landschaft auch sehr gut mit der generellen nation branding-Strategie Neuseeland. Zwar wäre es sicherlich eine Übertreibung, zu unterstellen, dass die Filmemacher direkt in Neuseelands nation branding-Projekt eingebunden oder gar dafür „bezahlt“ worden wären. Allerdings scheint die Werbung für die neuseeländische Landschaft auf dem DVDCover mehr auf potenzielle Touristen als auf Zuschauer abzuzielen. 
Tatsächlich sind die Authentisierungsstrategien im Falle von Good for Nothing von den Zuschauern insgesamt sehr gut angenommen worden. Lediglich in der Imdb-Rezension eines amerikanischen Zuschauers wird der Western-Handlungsort des Films aufgrund des Einsatzes nichtamerikansicher locations als explizit inauthentisch kritisiert:

I selected this film on TV completely blind. I had never heard of it, and was aware of none of the actors. Once the movie started, I was instantly aware that we weren't in the USA any more. The mountains didn't look right, and neither did the train. My first guess was Spain, except that the train wasn't right for that either. I finally gave in and looked up the film and learned that it was made in New Zealand. I realized then that it was made as homage to real Westerns, and the writers didn't have a clue („cseon“, 6. Oktober 2oI2).

Bemerkenswert ist hier, dass der Zuschauer bereits vor der Zuhilfenahme von Para- oder Metatexten allein beim Betrachten des Films die filmgeografische Inkongruenz anhand der Geomorphologie (Berge) zu erkennen vermochte. In diesem Fall differenziert der Zuschauer deshalb zwischen „echten“ Western und als „unecht“ beurteilten Western, deren locations offensichtlich nicht in den USA gewesen sind.

Dem steht jedoch eine Vielzahl anderer Rezensenten gegenüber, die den WesternHandlungsort gerade wegen des Einsatzes als „unberührt“ empfundener neuseeländischer locations als besonders authentisch und realistisch empfinden. So schreibt „fouuz-668-459294“ aus SüdFlorida in seiner Rezension: „Just not your ordinary western but a realistic look at some of the trappings of living in the 'old west'. [...] Make sure you see it with some one who enjoys these new realistic look at the west. Not some Hollywood set but out in the open wilderness" (24. April 2013). Der weitgehend on location erfolgte Dreh lässt für diesen Zuschauer die Landschaft als spürbar „real“ erscheinen, was für ihn mehr als ausgleichend für die Tatsache ist, dass nicht in den USA gedreht worden ist, sondern Neuseeland. Gerade mit Hollywood assoziiert dieser Zuschauer durch dessen häufigen Einsatz von Filmstudios Inauthentizität. Hingegen werden die neuseeländischen locations als authentisierender Faktor erachtet, während die Kenntnis über die filmgeografische Inkongruenz des Films als unproblematisch angesehen wird und nicht weiter auffällt, wie die Rezension von „nowego“ aus Australien demonstriert: „The music and cinematography of the film is first rate, up there with the best, I had trouble believing that the movie was shot in New Zealand“ (26. Mai 2012). 
Insgesamt unterscheidet sich Good for Notbing von den vorigen analysierten Filmen insbesondere durch die ungewöhnlich offensive Vermarktung der neuseeländischen locations als explizit hervorzuhebende unique selling proposition (USP) des Films und als Ausdruck der Filmemacher, dass Neuseeland mit der landschaftlichen Grandiosität des amerikanischen Südwestens konkurrieren könne. Durch diese offene Vermarktungsweise sehen die meisten Zuschauer auch keinen Grund, die Diskrepanz zwischen Handlungsort und location als „Vertragsbruch“ aufzufassen, da die geografische Inkongruenz gar nicht erst „verschwiegen“ wird. Einerseits stellt die Delokalisierung in Good for Notbing somit eine willed dislocation und Denationalisierung der eigenen Nationallandschaft dar, andererseits lässt sich an dem Umstand, das diese dislocation bewusst hervorgehoben wird, gleichzeitig auch Stolz auf die eigene Landschaft erblicken.

\subsubsection{Ergebnis}

Die Ausgangsfrage für die Analyse der dritten Simulationsstufe war: Welche filmgeografischen Konstruktions- und Authentisierungsstrategien setzen die Filmemacher ein, inwiefern werden diese Strategien von den Zuschauern akzeptiert und wie verhalten sich die Authentisierungsstrategien der Filmemacher gegenüber den Authentizitätserwartungen der Zuschauer?

Hierbei zeigte die Analyse, dass die Filmemacher vorwiegend folgende Authentisierungsstrategien einsetzten: Filmgeografische Delokalisierung, Projektion, Kompensation und (seltener) Autorisierung. Die Projektion der eigenen Sichtweise, dass die eingesetzten neuseeländischen locations den simulierten ausländischen Handlungsorten der jeweiligen Filme „verblüffend ähneln“ würden, auf die Zuschauer, war bei allen analysierten Filmen festzustellen. Eine weitere beliebte Strategie war die der Kompensation. Hierbei wurde argumentiert, dass die neuseeländische location zwar geografisch nicht mit dem ausländischen Setting übereinstimme, sich aber aufgrund seines vormodernen Aussehens besser zur Inszenierung eines historischen Handlungsortes eigne (und in dieser Hinsicht „authentischer“ sei) als die modernisierte Landschaft des eigentlichen Auslands. So argumentierten die Filmemacher von Last Samurai, dass die neuseeländischen locations aufgrund ihrer „Unberührtheit“ dem Zuschauer einen überzeugenderen Eindruck vom „Old Japan“ liefern würden als das modernisierte Japan selbst. Besonders explizit waren hierbei jedoch die Filmemacher des lokal produzierten Films Good for Notbing, wo nicht nur betont wurde, wie sehr die raue Otago-Landschaft dem Wilden Westen ähnele, sondern auch die neuseeländischen locations als unübersehbarer Teil der Vermarktungsstrategie des Films eingebunden wurden. 
Die Autorisierung durch einen Experten ist als weitere Strategie zu nennen, die bei Vertical Limit eingesetzt wurde: Durch die Involvierung eines Extrembergsteigers in die Filmproduktion sollte dem Zuschauer suggeriert werden, dass der Film in puncto Setting und Plot besonders realistisch sei. Diese Strategie läuft aber Gefahr, durch eine andere Autorität entwertet zu werden, wie dies bei Vertical Limit durch die Kritik Reinhold Messners an dem mangelnden Realismus des Films ersichtlich geworden ist.

Zumindest auf rudimentäre Weise haben die Filmemacher schließlich auch die neuseeländischen locations delokalisiert, um sie als ausländische Handlungsorte inszenieren zu können. Hierbei zeigte sich, dass die Delokalisierung sich bei vielen Filmen auf das gezielte Platzieren weniger auslandsspezifischer Marker wie Nationalflaggen oder das Einfügen eines ausländischen Berges in die neuseeländische Landschaft beschränkte. Dies deutet darauf hin, dass die Filmemacher die Tatsache ausnutzen wollten, dass die meisten Zuschauer ohnehin weder mit den geografischen Details der ausländischen Handlungsorte noch mit denen der neuseeländischen location vertraut sind und daher oft selbst größere geografische Diskrepanzen zwischen location und dem vom Handlungsort zu simulierenden Ausland zumindest auf den ersten Blick nicht erkennen können. Gleichzeitig verblieben durch die lediglich rudimentäre Delokalisierung aber auch immer erkennbare „Reste“ neuseelandspezifischer Artefakte in der Filmlandschaft, die von vielen Zuschauern als Zeichen filmgeografischer Inauthentizität gewertet wurden und bei ihnen sowohl Irritation, Verwirrung als auch Verständnislosigkeit hervorgerufen hat.

Insbesondere unter Zuschauern, die in dem von filmischen Handlungsort „repräsentierten“ Land wohnen, konnten viele auch ohne para-/metatextuelle Hintergrundinformationen die filmgeografische Inkongruenz während des Betrachtens des Films erkennen. Diese Zuschauer brachten insgesamt am wenigsten Verständnis dafür auf, dass nicht an Originalschauplätzen gedreht wurde und konnten sich in der Folge aufgrund der als inauthentisch empfundenen Handlungsorte kaum in das Filmgeschehen hineinversetzen.

Während die vorgenannte Zuschauergruppe demnach die neuseeländische location als deauthentisierenden Störfaktor empfand, waren neuseeländische Zuschauer hingegen oft der Ansicht, dass die neuseeländischen locations den Film aufwerten oder gar authentisieren würden, insbesondere, weil sie durch den hohen Wiedererkennungseffekt einen besonders starken lebensweltlichen Bezug zum Film aufbauen konnten. Des Weiteren zeigte sich vor allem bei der Analyse von Without a Paddle, dass neuseeländische Zuschauer das clean E green-Image ihres Landes reprodu- 
zieren und hiermit dazu beitragen, in Zeiten globaler Umweltprobleme Neuseeland als eine Art ökologisches Paradies zu stilisieren. Hiermit wird die weitverbreitete nostalgische Sehnsucht nach utopischen Räumen bedient - in diesem Falle einer ökologischen Utopie, die als Gegenentwurf zu der gerade durch die Globalisierung selbst hervorgerufenen oder zumindest verschärften Umweltverschmutzung dient.

In vielerlei Hinsicht zeigte sich auch, dass sich zwischen den Authentisierungsstrategien der Filmemacher und den Argumenten der neuseeländischen Zuschauer deutliche Parallelen finden lassen, die mit Neuseelands nation branding-Strategie konform gehen: Beide Gruppen beteuern, wie adäquat die neuseeländischen locations als „Doubles“ für die ausländischen Handlungsorte fungieren würden. Vor allem zeigte sich, dass sowohl die neuseeländischen Filmemacher von Good for Notbing als auch die meisten neuseeländischen Zuschauer stolz darauf sind, dass „ihre“ Landschaft in größeren Hollywoodproduktionen einem globalen Publikum präsentiert wird - wenn auch in mehr oder weniger „verschleierter“ Form.

Die Reaktionen nicht-neuseeländischer Zuschauer, die um den Einsatz neuseeländischer locations und somit um die geografische Inkongruenz wussten, war vielfältig: Während einige sich enttäuscht über die nicht gegebene Kongruenz zwischen location und Handlungsort zeigten und quasi-dokumentarische Ansprüche an die Filmgeografie dieser Filme hatten, empfanden andere den Handlungsort zwar als inauthentisch, waren aber geneigt, diesen Umstand zu akzeptieren, insbesondere wenn sie den Plot und/oder die schauspielerische Leistung als authentisch und ergreifend empfanden. Bei Vertical Limit, Without a Paddle und The Waterhorse: Legend of the Deep zeigte sich auch, dass ein Teil der Zuschauer sich sogar irritiert über das „Authentizitätsbedürfnis“ anderer Zuschauer zeigte und vielmehr der Ansicht war, dass Filme quasi naturgemäß inauthentisch und lediglich reines „Entertainment“ seien.

Bei Zuschauern, die nicht wussten, wo der jeweilige Film gedreht worden war, zeigte sich, dass ein signifikanter Teil hiervon auch heute noch in Zeiten beliebiger digitaler und anderweitiger Manipulierbarkeit der Filmlandschaft weiterhin gewohnheitsmäßig von einer Kongruenz von Handlungsort und location ausgeht. Dieser „leichtgläubige“ Zuschauertyp zeigt sich auch bei relativ aktuellen Filmen wie The Waterborse: Legend of the Deep überrascht, wenn er schließlich durch meta- oder paratextuelle Hintergrundinformationen von der geografischen Inkongruenz des Films erfahren muss. Zwar wird diese „Leichtgläubigkeit“ von einigen Zuschauern ironisch kommentiert, was eine gewisse Medienreflexivität und gestiegene Medienkompetenz erblicken lässt - dennoch ist 
der Anteil des „leichtgläubigen“ Zuschauertyps weiterhin erstaunlich hoch. Wenn dieser Zuschauertyp keine meta-/paratetextuellen Hintergrundinformationen als „Korrektiv“ heranzieht, kann dies eine stark verzerrte geografische Imagination zur Folge haben, bei der eigentlich neuseeländische Landschaftscharakteristika mit den vom filmischen Handlungsort „repräsentierten“ Ländern assoziiert werden. Mit anderen Worten sind solche Zuschauer geneigt, anzunehmen, dass beispielsweise der Loch Ness direkt ins Meer mündet, weil der Loch Ness in The Waterhorse: Legend of the Deep so dargestellt wurde.

Insgesamt hat die Analyse somit gezeigt, dass es einen lebhaften Diskurs über die filmgeografische Authentizität von Filmen der dritten Simulationsstufe gibt und dass die Authentisierungsstrategien der Filmemacher häufig inadäquat sind, um kritischere Zuschauer zu überzeugen. Dass der Authentizitätsdiskurs um die filmgeografische Authentizität der Filme auch Auswirkungen auf den Filmtourismus hat, hat insbesondere der Film The Waterhorse: Legend of the Deep gezeigt, der zwar filmtouristisches Interesse weckte, jedoch nicht für Neuseeland, sondern praktisch ausschließlich für Schottland. Dies bestätigt Tooke und Bakers These, dass Filmtouristen bei Filmen der 3. und 4. Simulationsstufe das Land besuchen wollen, das von den Handlungsorten dieser Filme „repräsentiert“ wurde, nicht jedoch das Land, wo diese Filme tatsächlich gedreht wurden. Ein Grund hierfür ist, dass die location als „inauthentisch“ erachtet wird, wenn das vom Handlungsort dargestellte Land ebenfalls real ist. Mit anderen Worten besuchen Filmtouristen lieber das „echte“ Loch Ness in Schottland als den „fake“-Loch Ness in Neuseeland.

Wenn demnach die filmtouristischen Ströme vorwiegend in das vom Handlungsort repräsentierte Land „gelenkt“ werden, kann man bei Filmen der 3. und 4. Simulationsstufe von einem Primat der Inhaltsebene sprechen, wohingegen die neuseeländische location als Störfaktor empfunden wird und hier kaum ein film tourism inducement nach Neuseeland auslösen kann. Diese Filme weisen demnach eine Doppelreferenzialität auf, bei der die beiden Referenzialitäten von Filmtouristen nicht als gleichwertig erachtet werden, sondern eine Hierarchie begründet wird. Hierin unterscheiden sich die Filme der 3. und 4. Simulationsstufe von den nun zu analysierenden Filmen der 5. Simulationsstufe, die von einer „alternativlosen“, singulären Referenzialität geprägt sind, da nur noch die locations, nicht aber die fiktiven Handlungsorte Referenzialität begründen können. 


\subsection{Analyse: V. und VII. Simulationsstufe}

\subsection{Willow (1988)}

Während mittlerweile fast allseits bekannt ist, dass die Lord of the Rings- und die HobbitTrilogien in Neuseeland gedreht wurden und seitdem Neuseeland als location für Fantasyfilme „entdeckt“ worden ist, handelt es sich hierbei keineswegs um die ersten in Neuseeland gedrehten Fantasyfilme. Vielmehr kann Willow, unter der Regie von Ron Howard und George Lucas als Produzenten, als der erste in Neuseeland gedrehte Fantasyfilm bezeichnet werden. Zwar wurde auch in Wales und den USA gedreht, ein Großteil der Dreharbeiten fand jedoch in Neuseeland statt. Neben der neuseeländischen location weist der Film jedoch auch deutliche Plotparallelen zu Lord of the Rings auf: Auch hier muss ein kleinwüchsiger Bewohner einer friedlichen agrarischen Siedlung gegen seinen Willen eine abenteuerliche Reise antreten und sich schließlich übermächtig erscheinenden Mächten stellen. Diese Entlehnungen, zusammen mit weiteren aus dem Neuen Testament und auch Lucas' eigener Star Wars-Trilogie, stellten damals einen der Hauptkritikpunkte an dem mäßig erfolgreichen Film dar.

Dennoch ist der Film auch bekannt für seine Innovativität hinsichtlich seiner digitalen visuellen Effekte, insbesondere einer sogenannten morphing-Sequenz sowie der ausgiebigen Nutzung des greenscreen-Projektionsverfahrens. ${ }^{70}$ Allerdings beziehen sich diese Effekte sämtlich auf die Charaktere und nicht auf die Landschaft. Die Landschaft wird zum größten Teil durch locations dargestellt, die praktisch ohne digitale Manipulation zu sehen sind. Im Vergleich zur heutigen Produktionsweise äußert sich Visual Effects Supervisor Dennis Muren in einem Interview rückblickend:

It's a completely different job to how it used to be. In some ways it's physically easier because you used to be on location, working with the director on all the shots, but there's less of that than there used to be. There were a lot of limits with what you could do with technology $[\ldots]$ (Muren, 20I3).

Muren weist darauf hin, dass die technischen Möglichkeiten zur Konstruktion einer Fantasywelt Ende der 1980er noch recht eingeschränkt waren, und dass sich solche Filmproduktionen aber mittlerweile von realen locations immer mehr wegbewegen und immer stärker dazu tendieren, zu reinen Studioproduktionen zu werden.

\footnotetext{
${ }^{70}$ Mit der morphing-Technik verwandelt sich ein Objekt scheinbar in ein anderes Objekt. Beispielsweise kann hierdurch das Gesicht eines Mannes in das einer Frau transformiert werden, wobei der Computer viele Zwischenstufen errechnet, z. B. von „90\% Mann:I0\% Frau“ hin zu „IO\% Mann:90\% Frau“ usf.
} 
Eine der wenigen Szenen, die am ehesten eine hypersublime und damit eine einer Fantasywelt „,angemessene“ Landschaftsästhetik aufweist, ist bezeichnenderweise auch eine der wenigen, die nicht on location gedreht wurde, sondern in einem Filmstudio, wobei die im Hintergrund zu sehende Landschaft nicht digital generiert, sondern wie damals üblich noch „analog“ durch das Malen eines fotorealistisch erscheinenden matte paintings bewerkstelligt wurde:

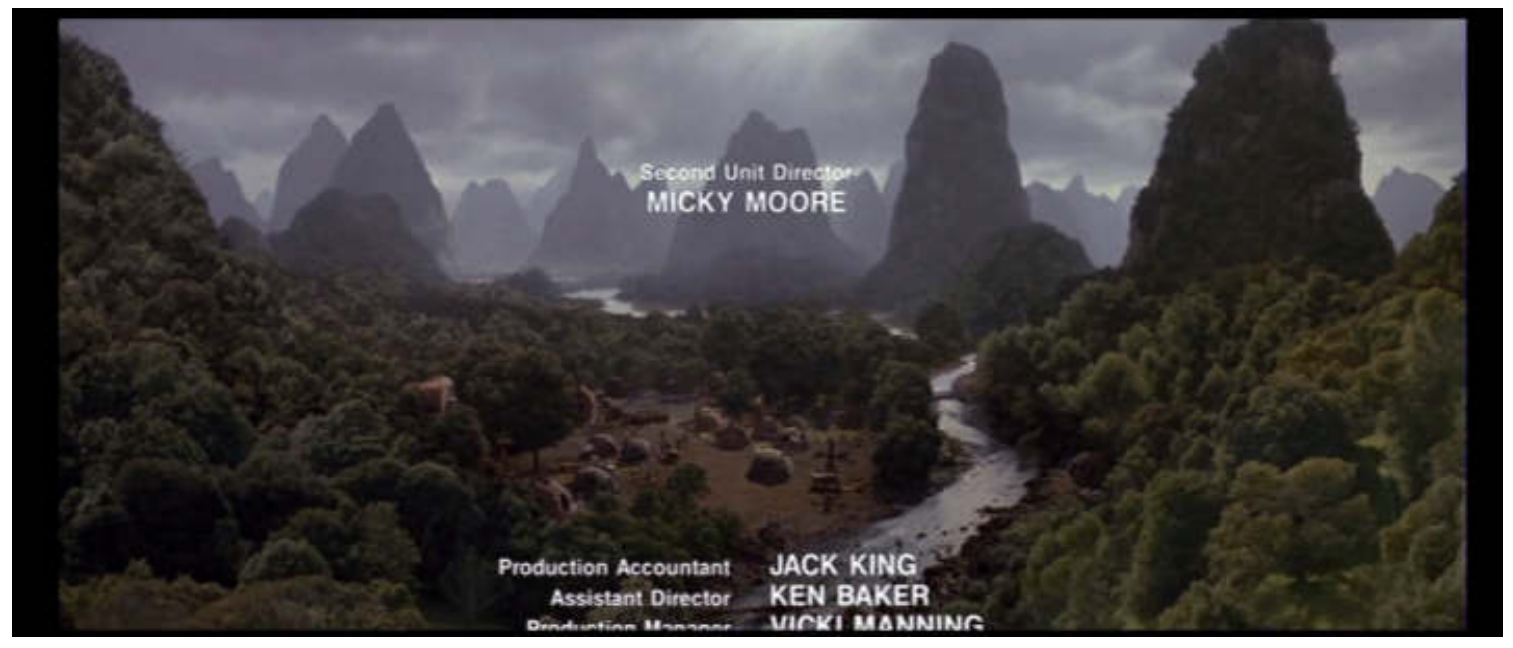

Abbildung 3.12: Endszene des Films Willow (Nelwyn-Tal) [02:08:19]

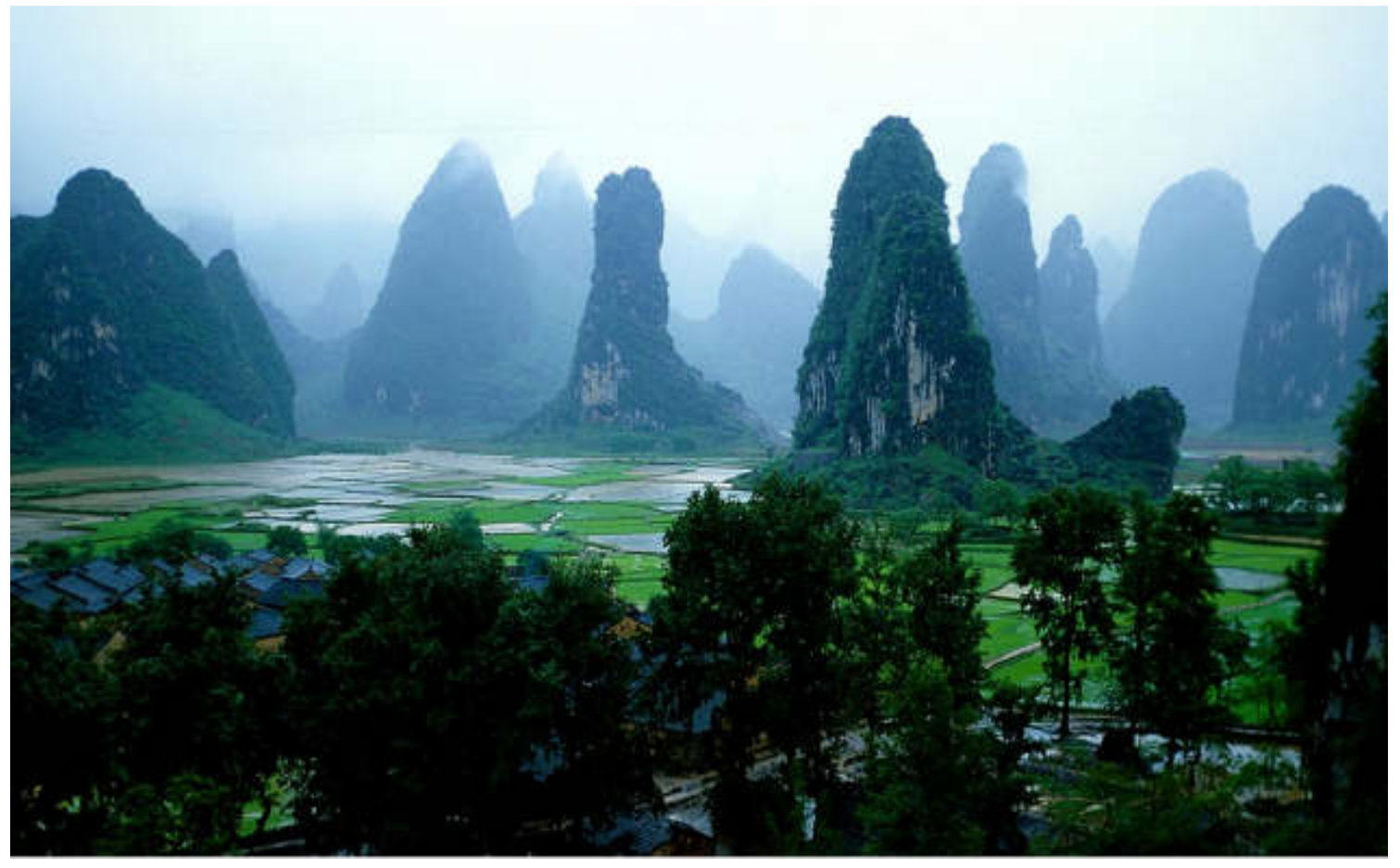

Abbildung 3.13: Guilin-Berge in China ${ }^{71}$

\footnotetext{
${ }^{71}$ Bildquelle: http://i.imgur.com/aQiRN.jpg (Zugriff: Is. Mai 20I5), Copyright: Alyson Strike
} 
Die in der Abbildung 3.12 zu sehende Filmeinstellung zeigt das Ende des Films, nach dem der Protagonist Willow unbeschadet von seinem großen Abenteuer in sein Nelwyn-Dorf heimgekehrt ist. Ein auffälliger Unterschied zu modernen Fantasyfilmen ist die eingeschränkte Beweglichkeit der Kamera: Sie führt in dieser Szene keine dynamischen Flugfahrten durch, sondern verharrt statisch in der gegebenen Perspektive. Die hierdurch für moderne Zuschauer als weniger immersiv empfundene Kameraführung ist bedingt durch das lediglich zweidimensionale matte painting im Hintergrund, was vor allem keine seitlichen Schwenks zulässt, da ansonsten unmittelbar die Zweidimensionalität der lediglich gemalten Landschaft offenbart und für den Zuschauer das Gefühl der Immersion zerstört würde.

Obwohl der Film grundsätzlich der fünften Simulationsstufe zuzuordnen ist, da primär on location gedreht wurde, handelt es sich bei der obigen Sequenz um eine Simulation siebter Stufe, da die Landschaft fiktiv ist und künstlich erzeugt wurde. Dennoch demonstriert Abbildung 3.13, die die chinesischen Guilin-Berge zeigt, dass die Landschaftskonstruktion in dieser Willow-Sequenz eindeutig von einer realen Geografie inspiriert worden ist. Die „Fantastik“ der Landschaft in dieser Sequenz begründet sich demnach auch weniger an der Sprengung physikalischer Gesetze, als vielmehr an der Auswahl besonders bizarrer irdischer Landschaften, die dann als Inspirationsquelle für den Film genutzt wurden. Zwar ist nicht on location in den Guilin-Bergen gedreht worden, die überaus deutliche Ikonizität zwischen der Willow-Sequenz und den Guilin-Bergen hat aber dennoch deutliches (theoretisches) Potenzial für den Filmtourismus, da ohne weiteres ein referenzieller Bezug zwischen Willow und China hergestellt werden kann. Dies illustriert, dass es bei der siebten Simulationsstufe immer auf den Einzelfall ankommt, d. h. wie sehr sich an realen Geografien orientiert wurde, ob daraus eventuell noch Filmtourismus generiert werden kann.

Wirklich charakteristisch für die Filmgeografie von Willow ist jedoch der Einsatz realer locations. Der Vorteil von on location-Drehs ist, wie im Methodikteil angeführt, der sich praktisch automatisch einstellende Realismuseffekt. Reale locations sind immer fotorealistisch, wohingegen bei digitalen Landschaften der Fotorealismus erst aufwendig berechnet werden muss. Zwar können matte paintings, wie sie in der Endszene von Willow eingesetzt wurden, ebenfalls fotorealistisch sein, aber auch dort ist die Erzeugung dieses Realismus aufwendig. Vor allem aber erlauben die on location-Szenen eine freie, dynamische Kameraführung, die die „Fantastik“ bzw. Außergewöhnlichkeit der Landschaft deutlich betonen kann. Wie wenig die locations in Willow nachbearbeitet wurden, illustrieren die folgenden Abbildungen: 

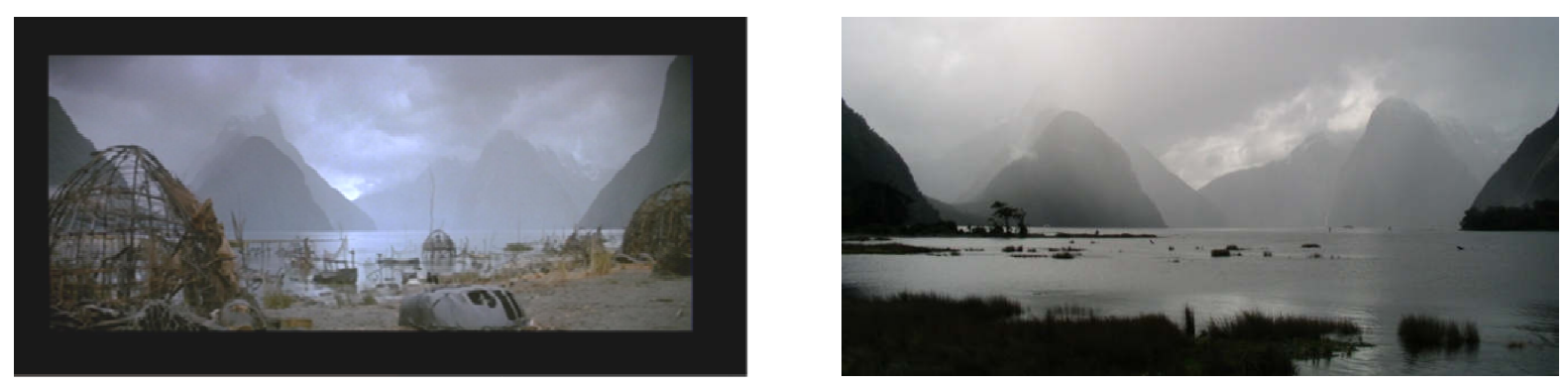

Abbildung 3.14: Vergleich I zwischen Filmszene aus Willow (links) und neuseeländischer location $^{72}$ (rechts)
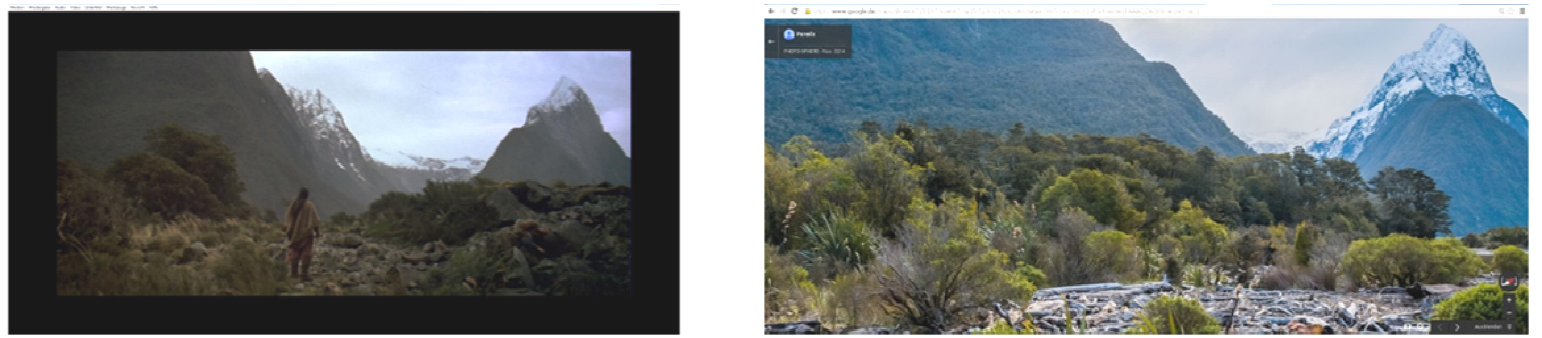

Abbildung 3.I5: Vergleich 2 zwischen Filmszene und neuseeländischer location (Google Streetview-Aufnahme [„Milford“])

Auf den Abbildungen ist links jeweils eine Filmszene zu sehen und rechts zum Vergleich die entsprechende nicht-manipulierte location des neuseeländischen Milford Sound. Abgesehen von einigen hinzugefügten rudimentären Holzbauten (Abbildung 3.I4 links), die dem Handlungsort eine archaische, vormoderne Atmosphäre verleihen sollen, ist offenkundig, dass die location praktisch unverändert zu sehen ist und somit die Ähnlichkeit bzw. Ikonizität zwischen Handlungsort und location außerordentlich hoch ist. Ein Filmtourist dürfte deshalb beim Besuchen der locations keinerlei Schwierigkeiten haben, eine „mental vision“ (Roesch 2009: I4I) der entsprechenden Filmszene zu haben, was den Film an sich für ein befriedigendes filmtouristisches Erlebnis prädestiniert. ${ }^{73}$

Allerdings kann bei derart geringfügiger Manipulation der locations die Problematik entstehen, dass Zuschauern der Handlungsort nicht „fantastisch“ genug erscheint, um als „authentische“ Fantasywelt überzeugen zu können, da diese gerade nicht einfach „irdisch“, sondern vielmehr „überirdisch“ erscheinen soll. Zudem verstärkt der Einsatz des Milford Sound, einer der bekanntes-

\footnotetext{
${ }^{72}$ https://farmi.staticflickr.com/II4/3II744933_af83077527_o.jpg (Zugriff: Is. Mai 20I5), Copyright: „Paul \& Kelly“ ${ }^{73}$ Die location des Milford Sound ist in Willow letztendlich genauso gut zu erkennen wie in Race for the Yankee Zephyr (198I), einem Film der I. Simulationsstufe, und damit einem Film, der gar nicht erst einen fiktiven, sondern einen „realen“ Handlungsort darstellen will.
} 
ten Touristenattraktionen Neuseelands, diesen Effekt der bloßen „Irdischkeit“ noch weiter, da ein besonders großer Teil der Zuschauer diese location beim Betrachten des Films unmittelbar wiedererkennen dürfte. So schreibt der Rezensent „Paul Andrews“:

With a supposed budget of $\$ 35,000$,ooo Willow is technically a very polished film with some terrific scenery, although it never really convinced me that it was set anywhere other than on Earth with it's snow covered mountain peaks, gravel pits \& the unspoilt natural beauty of Welsh \& New zealand forests that these types of fantasy films seem to use as locations (27. August 2005).

Trotz der relativ positiven Rezension hätte sich dieser Rezensent mehr (und nicht etwa weniger) Manipulation der Landschaft gewünscht, da sie ihm so nicht als wirklich überzeugende Fantasylandschaft erscheint. Für solche Zuschauer reicht demnach ein reiner on location-Dreh bei Fantasyfilmen nicht, die Landschaft muss vielmehr manipuliert werden, um als authentisch akzeptiert zu werden.

Für andere Zuschauer hingegen macht gerade die nur geringfügige Manipulation der Landschaften den Reiz des Films aus. User „Benjamin Cox“ schreibt: „This strange land of dwarfs, brownies and fairies feels much more believable because it looks real and it is somewhat alarming how quickly we have all got used to CGI in films“ (20. September 2005). Durch die fehlende digitale Manipulation wird für diesen Zuschauer die Fantasywelt erst authentisiert. Er äußert sich kritisch im Hinblick auf moderne Fantasyfilme wie Lord of the Rings, Chronicles of Narnia und Harry Potter, die ohne digitale Effekte nicht mehr auskommen. Während der Trend zur Digitalisierung sämtlicher Schritte einer Filmproduktion gerade bei Fantasyfilmen kaum rückgängig zu machen ist, sieht dieser Rezensent digitale Effekte nicht als authentisierende Hilfsmittel, sondern schreibt die allgemeine Akzeptanz digitaler Effekte vielmehr einem „Gewöhnungseffekt“ zu. Dass die Meinung der Zuschauer derart auseinandergeht, zeigt, dass kein wirklicher Konsens darüber herrscht, wie stark eine Fantasylandschaft manipuliert sein muss, um als glaubwürdig akzeptiert zu werden - wobei die allgemeine Akzeptanz von CGI in Fantasyfilmen darauf hindeutet, dass die CGIGegner mit ihrem Authentizitätsverständnis immer mehr zu einer Minderheit unter den Zuschauern werden.

Trotzdem die Filmlandschaft von Willow vielen Rezensenten reizvoll erscheint und obwohl die genauen Drehorte im Making Of kommuniziert wurden, hat Willow keinen signifikanten Filmtourismus nach Neuseeland oder Wales ausgelöst. Für den ausbleibenden Filmtourismus 
kommen meines Ermessens vor allem drei Gründe in Betracht: Zum einen wird aus den Rezensionen zwar durchaus ein Interesse an der Fantasywelt in Willow ersichtlich, jedoch keinesfalls die überschwängliche Begeisterung wie für die Lord of the Rings-Fantasywelt - mit anderen Worten war der Sehnsuchtsgrad nicht hoch genug. Gerade dieses Interesse muss aber sehr hoch sein, damit sich ein kleiner Teil der Zuschauerschaft überhaupt dazu entschließt, als Filmtouristen zur location zu reisen - zumal die Kosten für einen Urlaub in Neuseeland Ende der 1980er noch deutlich höher waren als heute.

Des Weiteren stellen die zahlreichen Entlehnungen aus den Tolkien-Romanen einen häufigen Kritikpunkt dar, der den Film in den Augen vieler Zuschauer als eine zweitrangige Kopie des damals noch als „unverfilmbar“ geltenden Lord of the Rings-Epos erscheinen ließ. Als dritter Punkt, der filmtouristische Aktivitäten erschwert hat, kann der Einsatz von locations aus verschiedenen Ländern (Neuseeland, Wales, USA) und Inspirationsquellen (China) angeführt werden, der ein vollständiges Nacherleben der Willow-Welt mit unverhältnismäßigem Aufwand verbunden hätte, da dafür eben nicht nur Neuseeland, sondern auch die anderen, weit voneinander entfernten locations besucht werden müssten. Dass letztendlich ein hoher Sehnsuchtsgrad wichtiger ist als ein hoher Ikonizitätsgrad, beweist die knapp 13 Jahre nach Willow erschienene Lord of the RingsTrilogie, bei der trotz der hochgradigen digitalen Manipulation vieler neuseeländischer locations (im Gegensatz zu Willow) ein Tourismusboom nach Neuseeland ausgelöst wurde, der das Selbstverständnis einer ganzen Nation verändern sollte.

\subsubsection{Lord of the Rings-Trilogie (2001-2003)}

Peter Jacksons Verfilmung der Lord of the Rings-Trilogie, die lange Zeit als „unverfilmbar“ galt, kann als eine der erfolgreichsten Fantasy-Filme in der Geschichte des Fantasy-Genres bezeichnet werden. Sowohl kommerziell als auch kritisch ist die Trilogie äußerst erfolgreich gewesen und hat zusammen mit der Harry Potter-Reihe ein bis dahin fast tot geglaubtes Genre wiederbelebt und einen zuvor nicht da gewesenen Fantasy-Boom mit Filmen wie Chronicles of Narnia (2005, 2008, 2010), Cloud Atlas (2012), Der Goldene Kompass (2007), Underworld(2003, 2006, 2012) und vielen weiteren Filmen ausgelöst. ${ }^{74}$ Gleichzeitig führte Jacksons Trilogie zu dem bislang einzigartigen Phänomen der Selbstvermarktung einer ganzen Nation als Handlungsort eines Films, namentlich „Mittelerde“, dem rein fiktiven Handlungsort der Lord of the Rings-Welt. Die Strategie, sich

\footnotetext{
${ }^{74}$ Lord of the Rings spielte weltweit über über I Mrd. USD ein und erhielt insgesamt I7 Oscars, darunter auch für visuelle Effekte.
} 
im Rahmen eines verstärkten nation brandings als „Home of Middle-Earth“ zu definieren, erwies sich als derart erfolgreich, dass sie sogar das nationale Selbstbild veränderte und eine Flut von Filmtouristen aus aller Welt anlockte. ${ }^{75}$

Während sowohl der kommerzielle und kritische Erfolg als auch die filmtouristischen Folgen der Lord of the Rings-Filme in einigen Forschungsarbeiten erörtert worden sind, ist die Rolle des sogenannten compositings, d. h. der möglichst nahtlosen Verschmelzung von CGI mit realen locations zwecks Erzeugung einer „authentisch“ erscheinenden Fantasywelt bislang nur am Rande erörtert worden. Die Rolle des compositings ist aber insofern wichtig, als dass sie ein zentraler Faktor ist, der dabei hilft, die fiktiven Welten in modernen Fantasyfilmen für Zuschauer zu authentisieren und dadurch die Sehnsucht nach diesen fiktiven Welten mitunter derart stark werden zu lassen, dass überhaupt erst Filmtourismus ausgelöst wird: Erst durch den Einsatz von CGI kann dem Anspruch heutiger Zuschauer nach Fantastik entsprochen werden, während gleichzeitig durch den Einsatz von locations noch ein realer Bezugspunkt für Zuschauer gegeben ist. Deshalb wird von Filmemachern trotz gelegentlich hervorgebrachter Zuschauer-Kritik an einem „Zuviel“ an CGI der CGI-Einsatz mittlerweile als unabdingbare Voraussetzung für den kommerziellen Erfolg moderner Fantasyfilme angesehen, wobei nicht nur der weitreichende Einsatz, sondern auch die „Qualität“ der CGI und des compositings von enormer Bedeutung ist, wie im Folgenden näher erörtert werden soll.

Bezüglich der Geeignetheit der location Neuseeland als Projektionsfläche für Mittelerde wendet Peter Jackson die Strategie der „Regionalanalogie“ (Zimmermann 2007: 34) an: So betont er im Vorwort zu Brodies The Lord of the Rings Location Guidebook, dass Neuseeland aufgrund seiner landschaftlichen Vielfalt und „Fantastik“ wie Mittelerde aussehe (2003: 6-7). ${ }^{76}$ Bei der Auswahl der locations wurde Wert darauf gelegt, die spektakulärsten Regionen auszuwählen, um der Zuschauererwartung nach sublimen Landschaften in Fantasywelten zu entgegenzukommen. Im Vergleich zu Willow wurde in Lord of the Rings aber wesentlich mehr CGI eingesetzt, das sich hier vor allem auch auf die Landschaftsdarstellung erstreckt. Gerade bei modernen Fantasyfilmen soll

\footnotetext{
${ }^{75}$ Hier ist allerdings auch anzumerken, dass Neuseeland zwar relativ schnell und effektiv auf die (unerwartete) Masse an Filmtouristen reagierte, das filmtouristische Potenzial aber keineswegs von vornherein antizipiert hatte, sondern anfangs mit Überraschtheit reagierte. Wie im Methodikteil unter Punkt 3.2.3.4 angeführt, kann das filmtouristische Potenzial von Filmen nicht zuverlässig prognostiziert werden.

${ }^{76}$ Dies stellt eine Parallele zur Authentisierungsstrategie der Filmemacher von Filmen der 3. und 4. Simulationsstufe dar, wo ähnlich argumentiert wird: So wurde beispielsweise wie angefuhrt in The Waterhorse: Legend of the Deep behauptet, dass Neuseeland und Schottland aufgrund ihrer (vermeintlichen) Ähnlichkeit „sisters“ sein könnten. Der Unterschied ist, dass hierbei zwei reale Geografien miteinander verglichen werden, während bei den hier behandelten Filmen der 5 . Simulationsstufe eine Ähnlichkeit zwischen fiktiver und realer Geografie unterstellt wird.
} 
durch die Kombination realer locations mit CGI der Eindruck einer fantastischen aber zugleich auch perzeptiv realistisch wirkenden Landschaft erzeugt werden. Peter Jackson betonte zu dieser Zeit noch, dass reale locations nicht nur für die Zuschauer, sondern auch für die Schauspieler einen „strong sense of the real“, also perzeptiven Realismus und „Authentizität“ gewährleisten würden (Jackson 2003: 7). Obwohl ein großer Teil der locations in Fellowship of the Ring, dem ersten Teil der Lord of the Rings-Trilogie, trotz des Einsatzes von CGI noch nicht derart verfremdet worden ist, dass die locations an sich nicht mehr zu erkennen wären, geht bereits hier der Einsatz von CGI stellenweise so weit, dass bestimmte Szenen auf die 7. Simulationsstufe vorausweisen, wie an der folgenden „Rivendell“-Szene ersichtlich wird:

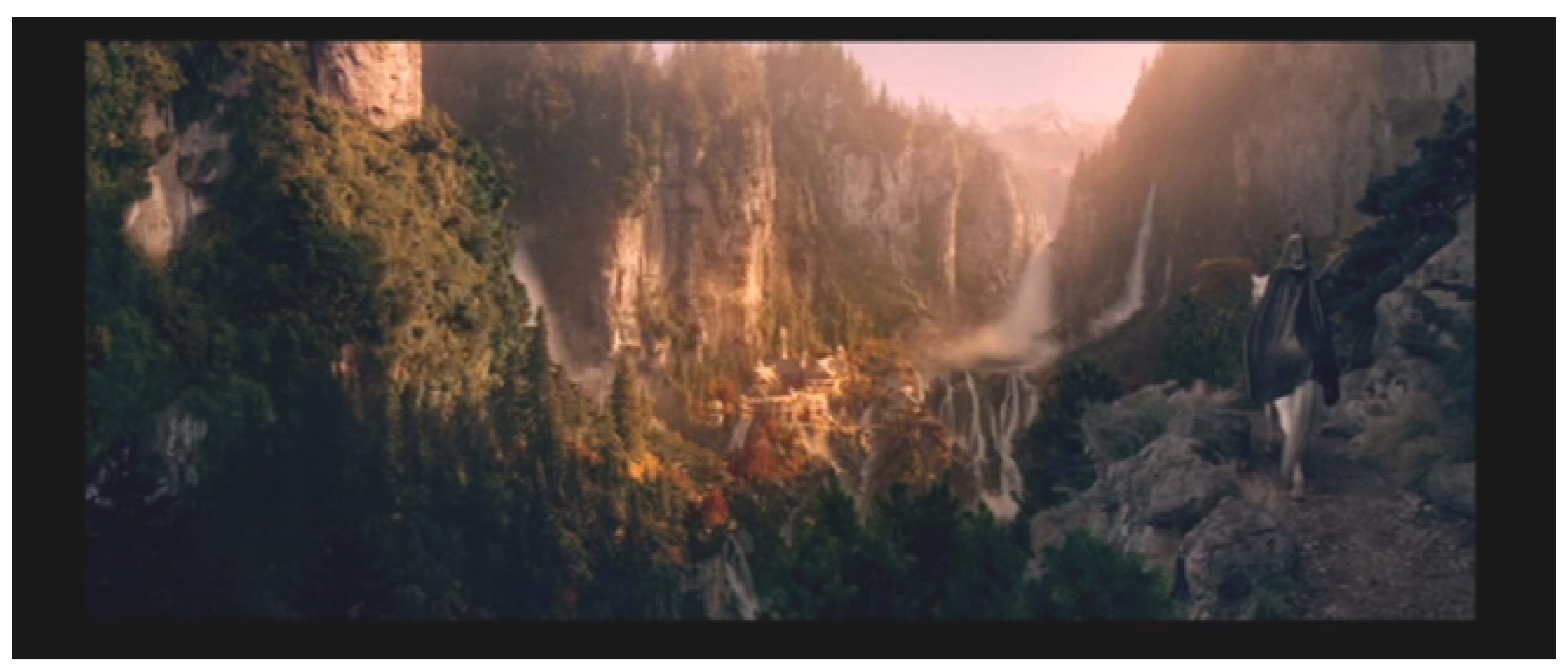

Abbildung 3.16: Das digital generierte Rivendell (Lord of the Rings: Fellowship of the Ring [or:12:49])

$\mathrm{Zu}$ sehen ist Rivendell, der Ort an dem sich sämtliche Protagonisten zum ersten Mal begegnen und die Gemeinschaft bzw. fellowship begründen, um den Ring nach Mordor zu bringen. Trotz der Beteuerung der Filmemacher, wie adäquat und fantastisch Neuseeland sei, konnte offenbar keine neuseeländische location gefunden werden, die den Ansprüchen der Filmemacher an eine zufriedenstellende Darstellung Rivendells gerecht werden konnte. Vielmehr handelt es sich hier um eine komplett digital generierte Landschaft, die als moderne Entsprechung der zuvor besprochenen Szene mit dem Nelwyn-Dorf in Willow mit den darin eingesetzten matte paintings angesehen werden kann, nur dass bei der Rivendell-Szene keine statische, sondern eine völlig dynamische Kameraführung eingesetzt wird, die Rivendell viel immersiver und „realer“ wirken lässt. Hierbei wurde 
auch Wert darauf gelegt, dass sich die Rivendell-Landschaft in ihrem perzeptiven Realismus nicht auffällig von demjenigen anderer Szenen, die unter Einsatz realer locations gedreht wurden, unterscheidet. Bemerkenswert ist hier, dass dies im Widerspruch zu Peter Jacksons oben zitierter Aussage steht, dass reale locations für Fotorealismus wichtig seien, wenn in Szenen wie der oben besprochenen unter Beweis gestellt wird, dass offenbar dieser Realismus auch ohne reale locations erreicht werden kann.

Da die Rivendell-Landschaft keine auch nur entfernte Entsprechung in der neuseeländischen Landschaft findet und folglich eine Entlokalisierung darstellt, kann auch keine entsprechende location von Filmtouristen in Neuseeland aufgesucht werden. Insofern werden Filmtouristen, die das Lord of the Rings Location Guidebook von Brodie zurate ziehen, mit dem Hinweis vertröstet, dass zwar das „eigentliche“ Rivendell in Neuseeland nicht zu finden sei, aber immerhin ein Set für die Szene, in der sich Frodo in einem Zimmer in Rivendell von seiner schweren Verwundung erholt, on location gedreht worden sei (2003: 5I). Weil es sich hierbei aber um eine Innenszene handelt, kann auch diese Szene nicht mit der neuseeländischen Landschaft assoziiert werden. Da auch bei der Darstellung Morias und Mordors - zwei weiteren, visuell besonders spektakulären Schauplätzen - mit einer Kombination aus CGI und Miniaturmodellen gearbeitet wurde, lässt sich daraus schlussfolgern, dass bei Lord of the Rings umso mehr CGI und umso weniger location eingesetzt wurde, desto spektakulärer und sublimer der Handlungsort sein sollte. Je mehr sich die Darstellung von einer irdischen Sublimität in eine „überirdische“ Hypersublimität verlagert, desto unabdingbarer wird der Einsatz von CGI folglich.

Auch bei der Simulation einer historischen Tiefe der Landschaft, die neben der Sublimität ebenfalls einen wichtigen Authentizitätsaspekt in Fantasyfilmen darstellt (wie im Methodikteil unter 3.2.3.2 erörtert), kam CGI im Film zum Einsatz. Die „junge“ neuseeländische Landschaft, der allgemein eine geringere „historische Tiefe“ als beispielsweise Großbritannien mit dessen zahlreichen historischen Relikten (Ruinen, Klöster usf.) zugesprochen wird, musste hierzu besonders stark manipuliert werden. Aber unabhängig davon, ob es sich um die Simulation kleinerer Ruinenfragmente oder um offensichtlich konstruierte, gigantische mittelalterliche Festungen handelte, wurde in jedem Fall ein besonderes Augenmerk darauf gelegt, die Digitalität der historischen Artefakte unsichtbar zu machen, wie die folgende Szene mit den Argonath, zwei riesigen Steinfiguren, die eine Flussmündung des Anduin-Flusses bewachen, illustriert: 


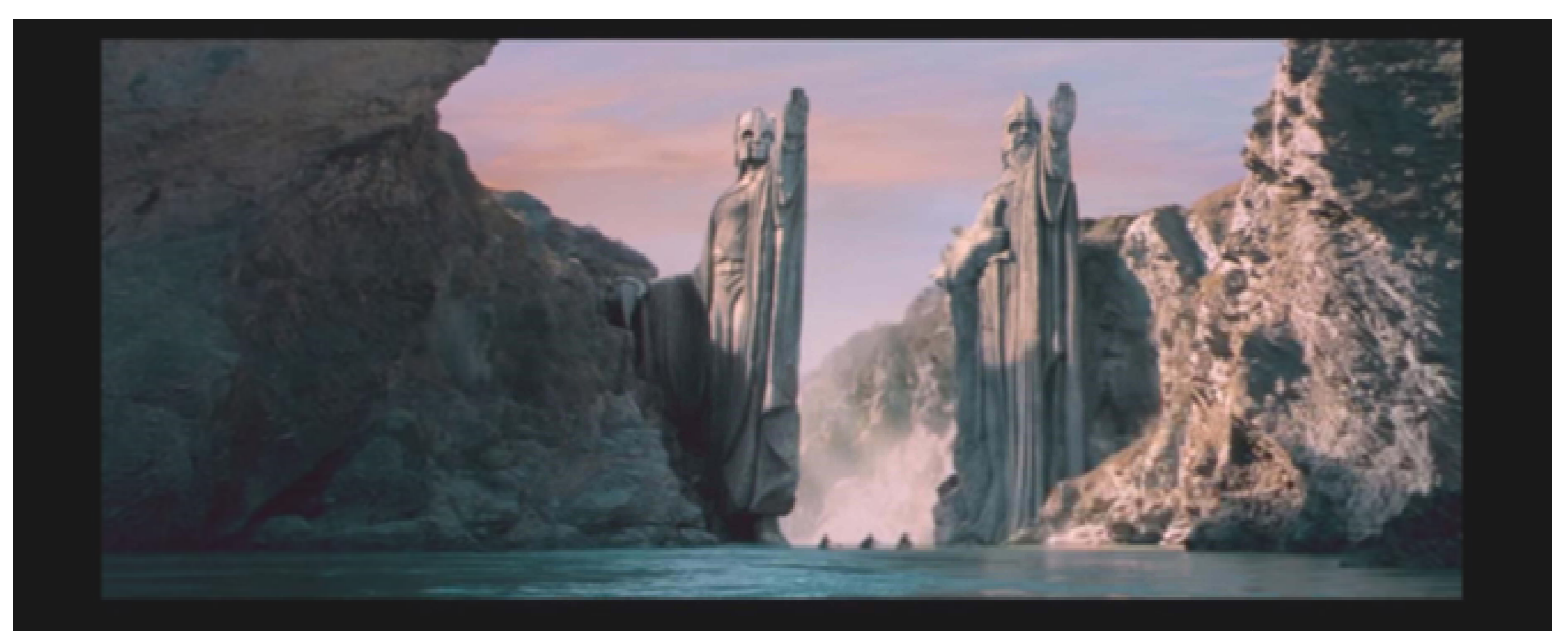

Abbildung 3.17: Argonath-Steinfiguren an Anduin-Flussmündung (Lord of the Rings: Fellowship of the Ring [02:18:32])

Wie deutlich ersichtlich wird, wurde Wert darauf gelegt, dass die Argonath, die eine Kombination aus CGI und Miniaturmodellen darstellen, sich nahtlos in die umgebende neuseeländische location einfügen. Sie fallen zwar durch ihren Gigantismus bzw. ihre Hypersublimität ins Auge, nicht jedoch durch einen wie auch immer gearteten visuellen Bruch zwischen ihnen und der sie umgebenden neuseeländischen location. Um dem Zuschauer zu suggerieren, dass die Argonath „real“ seien, wird vor einer dynamischen Kamerafahrt, die auch kleinste Details in einer Nahaufnahme zeigt, nicht zurückgeschreckt. Während früher digitale Modelle nur aus der Ferne gezeigt wurden, weil ihnen die für reale Objekte typischen Details fehlten, betonen heutige Filmemacher nicht ohne einen gewissen Stolz auch kleinste Details ihrer digital generierten Objekte, da diese mittlerweile derart fotorealistisch berechnet werden können, dass sie auch aus der Nähe perzeptiv realistisch wirken und von realen Objekten kaum zu unterscheiden sind. Hier spielt die hohe „Qualität“ der CGI eine ausgesprochen wichtige Rolle: Eine nicht-fotorealistische oder gar „verpixelte“ Darstellung der Argonath würde unmittelbar deren Digitalität und damit auch ihren (post-)modernen Produktionshintergrund für den Zuschauer klar erkennbar offenlegen und dadurch den beabsichtigten historischen Authentizitätseffekt vollständig unterwandern.

Die Kombination digitaler Bilder mit realen locations wurde von den Zuschauern generell sehr gut aufgenommen, wobei Rezensionen wie die folgenden häufig zu finden sind: 
New Zealand is Middle Earth. I was simply awed by the closesness of the two. The Shire is beautiful; it has that lush, green, homey sense Tolkien has in his books. Rivendell was also neat („,avrilyn“, 22. Dezember 200I).

The scenery $[\ldots]$ created was breathtaking when it needed to be, and foreboding when necessary, but always real, textured, and inspiring: The Shire was quaint and wondrous; Rivendell was majestic and picturesque [...] („Mike Stone“, 19. December 200I).

Aus diesen Rezensionen wird ersichtlich, dass Zuschauer zwar Mittelerde mit Neuseeland assoziieren und somit die von Jackson geäußerte Regionalanalogie akzeptieren, gleichzeitig aber nicht zwischen Handlungsorten, die auf realen locations basieren (5. Simulationsstufe) und digital generierten Handlungsorten (7. Simulationsstufe) differenzieren. In einem Atemzug werden sowohl die in Matamata gedrehten Shire-Szenen (deutsch: „Auenland“) als auch der vollständig digital generierte Schauplatz Rivendell (deutsch: „Bruchtal“) als gleichermaßen beeindruckend beschrieben. Daraus lässt sich schlussfolgern, dass reale locations anscheinend gar keinen so gewichtigen „Realitätsbonus" erzeugen, wie oft behauptet wird, da Zuschauer in diesem Zusammenhang gar nicht zwischen 5. und 7. Simulationsstufe differenzieren. Digitale Landschaften können folglich die Aufgabe realer locations mittlerweile adäquat übernehmen. Dass dennoch von vielen Zuschauern weiterhin auf reale locations Wert gelegt wird, obwohl die digitalen Landschaften kaum von den realen locations zu unterscheiden sind, legt nahe, dass es sich bei der Forderung nach realen locations vielmehr um ein vorwiegend psychologisches Bedürfnis mit einem ggf. daraus resultierenden „Placeboeffekt“ handelt und nicht um einen klar wahrnehmbaren visuellen Qualitätsunterschied, da Zuschauer nicht mehr sicher zwischen realen locations, digitalen Landschaften und diversen Kombinationen von beidem (compositing) differenzieren können.

Während ein großer Teil der Zuschauer das Augenmerk auf die „Qualität“ und den perzeptiven Realismus der digitalen Effekte legt und hierbei wie erwähnt bei Lord of the Rings zu einem vorwiegend positiven Urteil mit nur wenigen Kritikpunken kommt, ist das Urteil bezüglich der Adäquatheit der neuseeländischen location als Projektionsfläche für Mittelerde noch viel einhelliger positiv ausgefallen. Erstaunlicherweise findet sich in den Zuschauerrezensionen praktisch keine Forderung nach einer location, die näher an Tolkiens eigentlicher „Vision“ Mittelerdes gewesen wäre: Tolkiens Inspirationsquelle bei seiner Imaginierung Mittelerdes ist zu keiner Zeit Neuseeland 
gewesen, vielmehr bezog er seine Inspirationen aus europäischen Ländern, insbesondere Großbritannien (z. B. Hobbiton), Schweiz (insb. Rivendell), Skandinavien und Island (insb. Mordor), wie Ed Power in seiner Filmkritik „New Zealand is not Middle-Earth“ anführt. Nach Powers Ansicht nach könne ein so ,junges“ Land wie Neuseeland mit seiner „unblemished freshness“ nicht die „aura of decay“ widerspiegeln, die Tolkien in seinen Romanen beschreibt. Als positive Gegenbeispiele nennt Power die Games of Thrones-Serie, die in verschiedenen osteuropäischen Ländern gefilmt wurde und den in Irland gedrehten Klassiker Excalibur (198I). Über den Lord of the Rings- als auch den Hobbit-Dreh resümiert er: „Jackson ought to have gone to the Old World, to Ireland or Iceland and other places thereabout, where people seldom bungee-jump and the ancient and the modern often appear to live in uneasy alliance“ (Power, 2013).

In diesem Zusammenhang findet sich auch im Lord of the Rings Location Guidebook der Hinweis, dass das Tolkien-Anwesen die Publikation des Location Guidebook zwar genehmigt habe, aber keineswegs gutheiße (,published with the permission but not the approval of the estate of the late J. R. R. Tolkien“, Brodie 2003: 4), was ebenfalls darauf hindeutet, dass die Tolkien-Erben sowohl die kommerziellen Vermarktungsinteressen als auch die Suggerierung, Neuseeland sei das „reale“ Mittelerde, verurteilen. Ob ein Filmdreh in Europa von den Tolkien-Erben besser aufgenommen worden wäre, darüber lässt sich natürlich nur spekulieren. Es erscheint mir jedoch plausibel, dass dies trotz der dann immer noch gegebenen Kritik an kommerzieller „Ausschlachtung“ besser aufgenommen worden wäre, da diese näher an Tolkiens eigentlichen Inspirationsquellen gewesen wäre. Mit der Kritik an Neuseeland als „historisch inauthentischer“ Projektionsfläche gehören sowohl Power als auch die Tolkien-Erben aber definitiv einer Minderheit an, da generell klar ersichtlich ist, dass die Authentisierungsstrategie Peter Jacksons und die Selbstvermarktung Neuseelands als Mittelerde von einer überwältigenden Mehrheit akzeptiert oder gar als besonders adäquat beurteilt worden ist. Dies deutet darauf hin, dass Zuschauer bei der location-Auswahl bei Filmen mit fiktiven Handlungsorten weniger kritisch sind bzw. kaum Authentizitätsprobleme sehen und eine wesentlich breitere Auswahl an locations als Referenten zu akzeptieren bereit sind, als bei den zuvor analysierten Filmen der 3. und 4. Simulationsstufe.

Hinsichtlich eines film tourism inducement ist Lord of the Rings zweifelsohne sehr erfolgreich gewesen. In zahlreichen Rezensionen ist deutlich der hohe Sehnsuchtsgrad nach Mittelerde zu vernehmen. Diese Sehnsucht wird konkret auf Neuseeland projiziert, da Neuseeland durch seine „aggressive“ Marketingkampagne seine locations überdeutlich kommuniziert und dadurch erreicht 
hat, dass Neuseeland und Mittelerde fest miteinander assoziiert werden. ${ }^{77}$ Ein Werbeslogan der neuseeländischen Tourismusindustrie lautet „New Zealand, Home of Middle-earth“ und suggeriert damit eine Ähnlichkeit zwischen einem realen und einem fiktiven Ort. Hier stellt sich aber die Frage, wie (visuell) ähnlich Neuseeland und das filmisch konstruierte Mittelerde überhaupt sind, d. h. wie hoch der Grad an Ikonizität ist, da eine zu geringe Ähnlichkeit zwischen Handlungsort und location das filmtouristische Erlebnis beeinträchtigen kann. Dazu sollen im Folgenden drei Szenen exemplarisch analysiert werden.
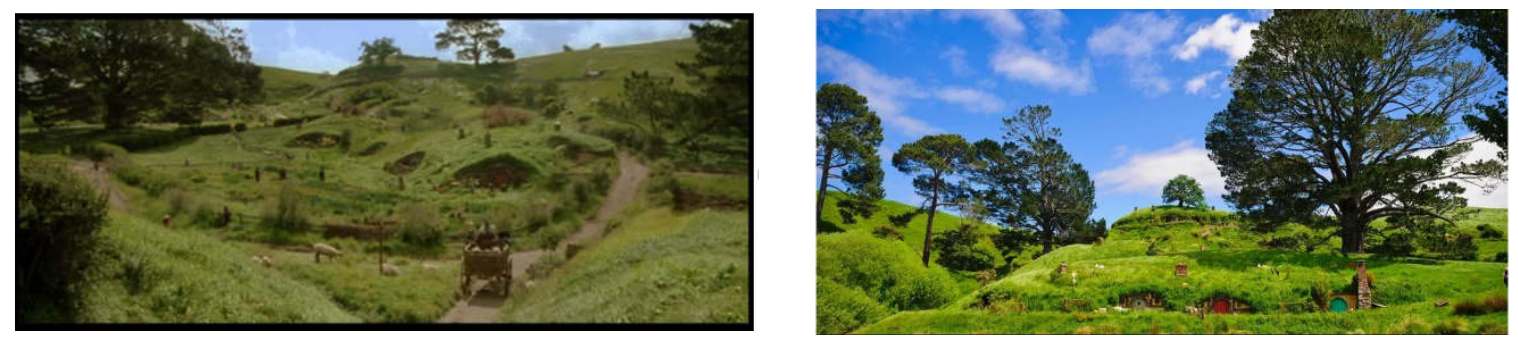

Abbildung 3.18: Szene I: Hobbiton (Lord of the Rings: Fellowship of the Ring [00:09:32]) und Matamata-location ${ }^{78}$

Die location Matamata, die zur Simulation des Handlungsortes Hobbingen dient, ist die bekannteste und bestbesuchte location, stellt aber gleichzeitig einen für den Film einmaligen Sonderfall dar: Die Landschaft wurde hier nicht etwa digital manipuliert, sondern allein durch den Bau eines Filmsets verändert. Im Gegensatz zu allen anderen im Film eingesetzten Filmsets ist das Hobbingen-Set nicht wieder abgebaut worden und hat damit die neuseeländische location dauerhaft physisch verändert. ${ }^{79}$ Hierdurch ist für Filmtouristen ein außergewöhnlich hoher Grad an Ähnlichkeit bzw. Ikonizität zwischen location und Handlungsort gegeben. Wie Abbildung 3.I8 zeigt, können Touristen ohne weiteres die „Hobbit-Höhlen“ in der Matamata-location vorfinden, was ein besonders „hyperreales“ Erlebnis gewährleistet, da ein vormals rein fiktiver Ort gewissermaßen physisch „realisiert“ worden ist. Die Touristen können demnach ähnlich wie in Disneyland

\footnotetext{
${ }^{77}$ Leotta führt an, dass durch das 9/II-Trauma die Sehnsucht nach utopischen Orten besonders groß geworden sei und Neuseeland aufgrund seiner Abgeschiedenheit als utopisch idealisiert werden konnte - Leotta spricht hierbei von einem „heterotopian impulse“ - wobei die Trilogie Lord of the Rings quasi der „Trigger“ gewesen sei (20Ir: 164). Ein Einwand gegen diese grundsätzlich plausible Argumentation ist meines Ermessens darin zu sehen, dass weder Mittelerde noch Neuseeland als rein utopisch dargestellt werden, sondern durchaus gewichtige, eher dystopische gothic-Elemente zu finden sind.

${ }^{78} \mathrm{http}: /$ www.newzealand.com/de/plan/business/hobbiton-movie-set-tours/(Zugriff: 16. Juli 20I5), Copyright: New Zealand Tourism

${ }^{79}$ Ein Grund, weshalb das Set nicht wieder abgebaut wurde, war, dass das Set auf einem Privatgelände errichtet worden ist, wo die strengen Umweltschutzbestimmungen des Department of Conversation - unter anderem das Abbauen von Sets und Wiederherstellen des „Ursprungszustandes“ der Landschaft - nicht eingehalten werden mussten.
} 
einen eigentlich fiktiven und daher immateriellen Ort „anfassen“. In gewissem Sinne könnte hier von einem „Tod des Realen“ gesprochen werden, da Teile des „realen“ Matamatas durch eine dauerhaft vorhandene „Simulation“ ersetzt worden sind, die für die Filmtouristen bedeutungsvoller ist als das „reale“ Matamata.

Während die Matamata-location einen außergewöhnlich hohen Grad an Ikonizität aufweist, handelt es sich hierbei wie erwähnt um einen Einzelfall; viel häufiger sind insbesondere im ersten Teil der Trilogie (Fellowship of the Ring) Szenen wie folgende anzutreffen:
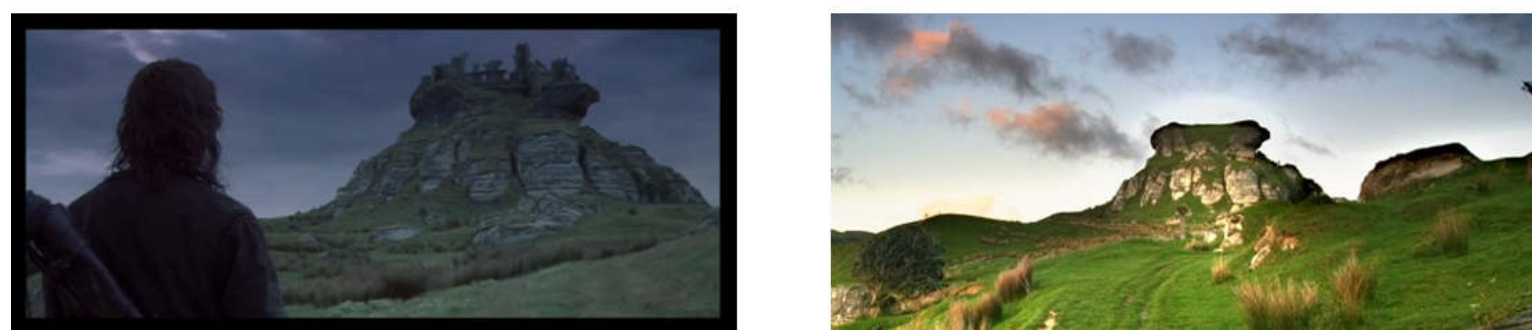

Abbildung 3.19: Szene 2: Weathertop (Lord of the Rings: Fellowship of the Ring [00:58:04]) / Port Waikato-location ${ }^{80}$

$\mathrm{Zu}$ sehen ist links die Filmszene, in der Aragorn auf die Wetterspitze blickt und in der er und die Hobbits Zuflucht vor Saurons Schergen suchen, nachdem sie Hobbingen und Bree verlassen haben. Rechts ist die eigentliche location in Neuseeland zu sehen. Auch hier ist der Ikonizitätsgrad recht hoch, wobei durch das abgebaute Filmset dem Filmtouristen bereits mehr Fantasie zum Nacherleben der Filmszene abverlangt wird. Für den zweiten und insbesondere dritten Teil der Trilogie (Two Towers, Rückkebr des Königs) allerdings werden auch solche Szenen immer seltener und stattdessen Szenen des folgenden Typs immer häufiger:

\footnotetext{
${ }^{80}$ Bildquelle: www.weltwunderer.de/teil-3-der-herr-der-ringe-location-tour-rund-um-den-tongariro-national-park-mordor-co/ (Zugriff: 16. Juni 20Is), Copyright: „Weltwunderfrau“
} 

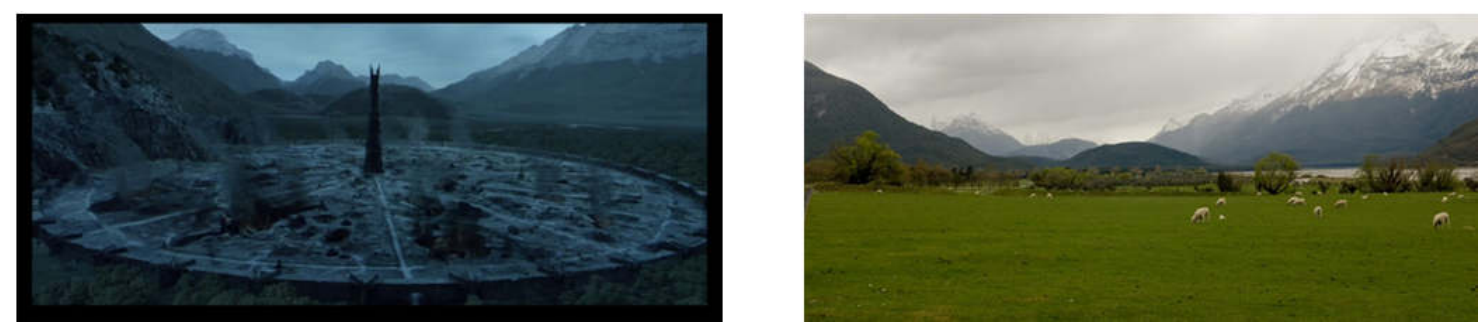

Abbildung 3.20: Szene 3: Isengart (Lord of the Rings: Fellowship of the Ring [02:40:00]) / Glenorchy-location ${ }^{8 I}$

In Abbildung 3.20 ist links die Filmszene mit dem in die Dunkelheit verfallenen Isengart zu sehen und rechts die eigentliche location Glenorchy. Im Gegensatz zu den zuvor behandelten Szenen ist hier der Ikonizitätsgrad derart gering, dass es für Filmtouristen kaum mehr möglich ist, die Filmszene beim Betrachten der location wirklich nacherleben zu können. Während das filmische Isengart von einer ausgesprochen dunklen gotbic-Ästhetik geprägt ist und der Isengart-Turm sowie die kreisrunde Umgebung eine Mischung aus CGI und Miniaturmodell darstellen, ist davon in der eher pastoral anmutenden Glenorchy-location nichts mehr zu sehen. Nur anhand der ähnlichen Geomorphologie im Hintergrund lässt sich noch ein relativ vager Zusammenhang zwischen Filmszene und location herstellen.

In Gesamtbetrachtung zeigt sich, dass durch den intensiven Einsatz visueller Effekte ein Großteil der locations in der Lord of the Rings-Trilogie derart verändert worden ist, dass die eigentliche Charakteristik der locations hinter den filmischen Handlungsorten stark verblasst ist. Wichtige Handlungsorte wie Rivendell, Isengart und Mordor haben aufgrund ihrer starken CGI-Lastigkeit kaum oder keine Ähnlichkeit mit Neuseeland mehr. Durch den intensiven Einsatz digitaler visueller Effekte ist der Ikonizitätsgrad im Vergleich zum zuvor analysierten Film Willow ungleich geringer. Mittelerde hat somit visuell wesentlich weniger mit Neuseeland gemeinsam als der nicht benannte Handlungsort von Willow. Daraus ergeben sich zwei Konsequenzen: Zum einen deutet es darauf hin, dass der Ikonizitätsgrad erstaunlich gering sein und dennoch Filmtourismus auslösen kann (zumindest bei Fantasyfilmen). Zum anderen weist Roesch darauf hin, dass verringerte Ikonizität das filmtouristische Erlebnis erheblich beeinträchtigen kann. So berichtet Roesch von einem Jugendlichen, der darüber enttäuscht war, dass die Argonath-Statuen nur digital eingefügt worden

\footnotetext{
${ }^{81}$ http://neuseeland.your-photo.net/home/index.php?id=I2\#menu (Zugriff: I4. Juni 2oIs), Copyright: „Sebastian“
} 
waren. Aber diese Überraschtheit und Enttäuschung beschränkte sich keineswegs auf „naive“ Kinder und Jugendliche; auch Erwachsene wussten zwar um die grundsätzliche CGI-Manipulation, hatten aber den Grad der Manipulation unter- und den Grad der Ikonizität überschätzt. So zitiert Roesch eine erwachsene Filmtouristin, die an einer Lord of the Rings-Tour teilnahm: „I thought that was a bit of a pity. I was probably wishing for purer landscapes, less digital enhancement because it was not really necessary“ (2009: 152). Ein durch geringe oder fehlende Ikonizität verhinderter Wiedererkennungseffekt machte daher ein mentales Nacherleben der Filmszene bzw. eine „mental vision“, wie Roesch es bezeichnet, für diese Filmtouristen nur schwer möglich und damit das Erlebnis wenig befriedigend. Daraus kann man schlussfolgern, dass Zuschauer bei der filmischen Darstellung von Fantasywelten meist einen erheblichen Einsatz von CGI voraussetzen, um diese Fantasywelt als authentisch akzeptieren zu können, dass sie sich vor Ort als Filmtouristen aber wünschten, es wäre gerade nicht so viel CGI eingesetzt worden, weil ihnen durch den geringen Ikonizitätsgrad nun die reale location weniger authentisch vorkommt - und dass, obwohl es genau der intensive Einsatz von CGI war, der ihr filmtouristisches Interesse weckte.

In diesem Zusammenhang weist Carl auf die wichtige Rolle der touristischen Guides, d.h. Personen, die die Touristen auf die exakten locations hinweisen, hin, und stellt eine direkte Korrelation zwischen der Zufriedenheit der Touristen und der „Qualität“ der Guides fest: Desto weniger vom Handlungsort in der location wiederzuerkennen ist, desto wichtiger sind die Guides, die die Filmtouristen auch auf kleinste Ähnlichkeiten zwischen location und Handlungsort hinweisen, die diese ohne die Guides nicht erkannt hätten (2004: 148). Dass die Guides explizit auf „Ikonizitätspuren“ hinweisen müssen, illustriert, wie stark die digitale Manipulation der Handlungsorte gewesen ist und wie wenig noch von der eigentlichen location für die Filmtouristen erkennbar bleibt. Dies wiederum legt den Schluss nahe, dass Filmtourismus trotz des geringen Ikonizitätgrades oftmals nicht so sehr deshalb ausgelöst wird, weil den Filmtouristen die tatsächliche visuelle Übereinstimmung von location und Handlungsort gleichgültig wäre, sondern vielmehr weil sie im Film eben nicht mehr sicher zwischen location, CGI und Modellen/Sets differenzieren können und aufgrund des perzeptiven Realismus des Films dazu neigen, mehr reale locations bzw. geografische Realität im Film zu vermuten, als dort wirklich gegeben ist. CGI erzeugt demnach bei Zuschauern - trotz deren genereller Kenntnis, dass CGI eingesetzt wurde - unrealistische und überhöhte Erwartungen an reale Geografien. 


\subsubsection{Hobbit-Trilogie (2010-2012)}

Fünf Jahre nach King Kong und ein Jahr nach Avatar, beides Filmen der 7. Simulationsstufe, erschien der erste Hobbit-Teil An Unexpected Journey als Prequel zur Lord of the RingsTrilogie. Während bereits bei Lord of the Rings eine Zunahme von CGI von Fellowship of the Ring zu Return of the Ring festzustellen war, hat sich der CGI-Anteil in der Hobbit-Trilogie noch einmal deutlich erhöht und von Folge zu Folge weiter gesteigert. Da noch reale locations eingesetzt wurden, wird der Film hier noch der 5 . Simulationsstufe zugeordnet, er tendiert insgesamt aber bereits deutlich zur 7. Simulationsstufe, was unter anderem auf den Einfluss von King Kong und Avatar zurückzuführen ist. Während in der Lord of the Rings-Trilogie bereits die weitgehend digitalen Landschaften Rivendells, Morias und Mordors einen nicht unerheblichen Teil der Spielzeit des Films ausmachten, machen die digitalen Handlungsorte Goblin Caves, Esgaroth, City of Dale, Dol Guldur und Gundabad in der Hobbit-Trilogie einen noch deutlich größeren Teil der Filmspielzeit aus. Insbesondere in Desolation of Smang, dem zweiten Teil der Hobbit-Trilogie, alterniert das Geschehen in der zweiten Filmhälfte praktisch nur noch zwischen der Seestadt Esgaroth und der City of Dale, deren Darstellungen ohne reale locations auskommen. ${ }^{82}$

Parallel zur Zunahme der CGI und Abnahme der locations verfolgt Peter Jackson beim Hobbit eine Authentisierungsstrategie, die sich von derjenigen bei Lord of the Rings deutlich unterscheidet: Das Ziel, die Fantasywelt immersiver und authentischer wirken zu lassen und zudem beim Zuschauer das Gefühl zu wecken, etwas Neues zu sehen, soll nun durch den verstärkten Einsatz von CGI in Kombination mit moderner 3 D- und sogenannter HFR-Technologie ${ }^{83}$ erreicht werden, während reale locations in ihrer Authentisierungsfunktion nur mehr eine Nebenrolle spielen. Außerdem werden aufgrund der 3 D-Technologie keine Miniaturmodelle mehr zur Simulation landschaftlicher Historizität eingesetzt, da diese hierbei zu „modellhaft“ erscheinen würden. Hier kann also erst recht von einer „digitalen Historizität“ gesprochen werden, die im Gegensatz zu Lord of the Rings (beispielsweise die besprochenen Argonath-Statuen) keinerlei physische Realität mehr aufweist.

\footnotetext{
${ }^{82}$ Zwar ist in der Hobbit-Trilogie für das Hobbiton-Filmset auch diesmal wieder ein physisches Set konstruiert worden, wobei es diesmal sogar aus echtem Holz gebaut wurde; dies geschah jedoch nicht aus Gründen des Realismus, sondern ergab sich aus einer Verhandlung zwischen der Alexander Farm und dem Filmproduzenten Warner Bros. und stellt damit vielmehr ein touristisches Zugeständnis dar.

${ }^{83}$ HFR bedeutet High Frame Rate und bezeichnet Filme, die statt der üblichen 24 Bilder pro Sekunde mit 48 Bildern pro Sekunde gefilmt werden und dadurch auch schnelle Bewegungen ohne Bewegungsunschärfe oder „Schlieren“zeigen können, wodurch mehr Details zu sehen sind.
} 
Statt wie bei der Lord of the Rings-Produktion die Außenszenen größtenteils on location zu drehen, ist die Hobbit-Trilogie folglich eine viel studiolastigere Produktion, wo nur noch dort on location gedreht wurde, wo es „notwendig“ erschien. Dies ist eine Strategie, die sich deutlich von derjenigen beim Dreh von Lord of the Rings unterscheidet, wo noch vom immensen Aufwand bei den zahlreichen on location-Drehs berichtet wurde und diese als prägend für die Authentizität der Fantasywelt dargestellt wurden. Der Einsatz von on location-Drehs beim Hobbit lässt auf den ersten Blick zwar vermuten, dass deren Notwendigkeit wie schon bei Lord of the Rings in erster Linie im perzeptiven Realismus begründet liegt. Hierbei stellt sich aber die Frage, weshalb der fünf Jahre vor dem Hobbit erfolgte Dreh von Peter Jacksons King Kong überhaupt keine locations mehr erforderte und dennoch ja auch dort perzeptiver Realismus angestrebt wurde? Dies legt den Verdacht nahe, dass beim Hobbit weniger aus Gründen des perzeptiven Realismus, sondern vielmehr aus anderen Gründen neuseeländische locations eingesetzt wurden: Zum einen, um das filmtouristische Potenzial des Films und damit die nation branding-Strategie „Home of Middle-Earth“ nicht zu gefährden und zum anderen, um die neuseeländische Nation nicht zu konsternieren.

Dies wird vor allem vor dem Hintergrund der sogenannten Hobbit-Krise ersichtlich, wo Neuseeland nicht nur Arbeitsplätze in seiner Filmindustrie, sondern auch seinen Filmtourismus und sein Image als „Mittelerde“ gefährdet sah: Der von Jonathan Handel in seinem Buch The New Zealand Hobbit Crisis eingehend erörterte Konflikt im Jahre 2010 zwischen neuseeländischen Schauspielern und Warner Bros. hatte folgenden Ablauf: Als neuseeländische Schauspieler der Aufforderung der $\mathrm{MEAA}^{84}$ nachkommen, die Mitarbeit am ersten Hobbit-Film zu boykottieren, um damit bessere Arbeitsbedingungen einzufordern, sorgt dies für sichtliche Verärgerung beim Produzenten/Regisseur Peter Jackson und bei der Produktionsfirma Warner Bros. Während Jackson aufgrund seiner national/transnationalen „Doppelagenda“ eher subtile Andeutungen macht, die Produktion notfalls ins Ausland zu verlagern, aber klarstellt, dass dies für ihn nur eine Notlösung sei, droht Warner Bros. unverblümt, die Produktion nach Europa, vorzugsweise nach England, zu verlagern. Das Einlenken der neuseeländischen Regierung mit einer Gesetzesänderung quasi über Nacht, worin der Status der Schauspieler als „freiberuflich“ deklariert wird (2012: 5-7). Einer der Effekte der Globalisierung ist die Verschlechterung der Arbeitsbedingungen vieler Menschen, was sich hier an der faktischen Beschneidung der Rechte neuseeländischer Schauspieler aufgrund des transnationalen Agierens von Warner Bros. deutlich zeigt. Dass die neuseeländische Re-

\footnotetext{
${ }^{84}$ Australia's Media, Entertainment and Arts Alliance
} 
gierung sich dem Druck eines transnationalen Unternehmens gebeugt hat, zeigt, wie existenziell Neuseeland die Bedrohung empfand, weil sich mittlerweile herauskristallisiert hatte, dass der durch Lord of the Rings erzeugte Tourismusboom nicht langfristig über Jahrzehnte anhalten würde, sondern durch weitere Blockbuster wie The Hobbit das globale Interesse an Neuseeland als Filmtouristenziel regelmäßig erneuert werden muss. Hier hat Neuseeland demnach nur wenig Resistenz gegenüber den Globalisierungseinflüssen gezeigt und ein Stück seiner nationalen Souveränität eingebüßt.

Dass die Hobbit-Krise derart eskalieren konnte, liegt auch an den technologischen Fortschritten, die die Filmtechnologie mittlerweile gemacht hat: Durch die CGI-Technologie schreitet die Virtualisierung der locations immer weiter voran und entkoppelt damit zusehends den Handlungsort von einer physisch existenten location. Dies ermöglicht Filmemachern eine maximale Kontrolle über die Produktionsbedingungen beim on location-Dreh und eine zunehmende Emanzipierung von den Unwägbarkeiten einer physischen location (z. B. Wetterverhältnisse, störende Landschaftsartefakte, Drehgenehmigungen). Allen Beteuerungen der neuseeländischen Tourismusindustrie zum Trotz, dass Mittelerde und Neuseeland „untrennbar“ miteinander verschmolzen seien, hat die Entschlossenheit Warner Bros., die Produktion ggf. ins Ausland zu verlagern, demonstriert, dass Neuseeland letztendlich nur eine austauschbare Projektionsfläche bleibt, und die „Charakteristika“ Mittelerdes heutzutage bei Bedarf mittels CGI auch an einer beliebigen anderen location digital nachgebildet werden können. ${ }^{85}$ Aus diesem Grund stellt sich auch der neuseeländische Schauspieler Ian Mune, der in Lord of the Rings bereits als Hobbitdarsteller mitgewirkt hatte, gegen das seiner Ansicht nach übertriebene nationale Pathos Neuseelands („I don't think it is as substantial a piece of the New Zealand heart as some people would like to make out") und kommt zu dem Schluss, dass die Hobbit-Trilogie letztendlich überall gedreht werden könne: „[...] they could shoot it in South Africa, they could shoot it in [the Czech Republic], Romania, or somewhere deep in the heart of Kazakhstan, or if they weren't having a war they could probably shoot the thing in bloody Afghanistan“" (Masters, 2010).

Nach den außerordentlichen und letztendlich erfolgreichen Anstrengungen Neuseelands, die Hobbit-Produktion im Lande zu belassen, wäre die Entscheidung Peter Jacksons, die Trilogie

\footnotetext{
${ }^{8 s}$ Es wäre interessant gewesen zu sehen, ob eine Verlagerung des Drehs nach Europa Zuschauerkritik hervorgerufen hätte. Dies hätte Indizien dazu geliefert, wie sehr die Botschaft „Neuseeland ist Mittelerde“ von Zuschauern tatsächlich internalisiert worden ist. Da aber, wie in der Analyse zu Lord of the Rings angefuhrt, keine Kritik seitens der Zuschauer über die Wahl einer location, die nicht Tolkiens Vision bzw. Inspirationsquellen entsprochen haben, zu vernehmen war, hätte die globale Zuschauerschaft vermutlich trotz aller Marketingbemühungen seitens Neuseelands kein Problem mit einer nicht-neuseeländischen location gehabt.
} 
nun ähnlich wie King Kong oder Avatar vollständig digital und ohne den Einsatz neuseeländischer locations zu drehen, geradezu wie eine Verhöhnung erschienen. Es wurde ja nicht allein ein Vorteil für die neuseeländische Filmindustrie erhofft (was auch durch einen rein digitalen Dreh durchaus der Fall gewesen wäre), sondern auch für den Filmtourismus und nicht zuletzt das Selbstbewusstsein einer Nation. Ohne den Einsatz neuseeländischer locations wäre der Hobbit aber nicht mehr länger wie noch Lord of the Rings als eine Werbung für die landschaftliche Schönheit Neuseelands betrachtet worden und hätte womöglich ähnlich wie King Kong - wie in der nächsten Analyse erörtert werden wird - keinerlei Filmtourismus ausgelöst. Insofern erscheint die Notwendigkeit des Einsatzes realer (neuseeländischer!) locations aus Sicht der Filmemacher weniger eine des perzeptiven Realismus, sondern eine vor allem eine „moralische“ gewesen zu sein, da aus filmtouristischen Gesichtspunkten ein Filmdreh mit rein digitalen Landschaften ebenso ungünstig für Neuseeland gewesen wäre wie eine Verlagerung des Filmdrehs nach Osteuropa.

Der stark gestiegene Anteil digitaler Landschaften und Charaktere hat bei den Zuschauern gemischte Reaktionen hervorgerufen, wobei der Ton der negativen Rezensionen deutlicher schärfer ist als bei Lord of the Rings. Viele Zuschauer beklagen ein „Zuviel“ an CGI und bezeichnen die Hobbit-Welt als „cartoonhaft“ oder auch „computerspielähnlich“, was definitiv abwertend gemeint ist. Aber wie bei der Analyse von Lord of the Rings angeführt wurde, können Zuschauer meist gar nicht mehr sicher zwischen digitalen und „realen“ Objekten differenzieren, weshalb auch hier weniger von einem tatsächlichen „Erkennen“ digitaler Objekte als vielmehr von einer generellen Skepsis gegenüber der filmischen Darstellung gesprochen werden muss. Wenn der Hobbit-Darsteller Graham McTavish in dem touristischen Werbeclip „New Zealand, Home of Middle-earth“ davon spricht, dass Zuschauer davon ausgingen, die Landschaften im Hobbit seien „photoshopped“ („New Zealand, Home“ [oo:03:36-oo:03:44]), gleichzeitig aber darauf hinweist, dass diese Berge tatsächliche neuseeländische Berge seien, dann zeigt sich hieran nicht nur, dass viele Zuschauer mittlerweile mit einer präventiven Skepsis bezüglich des Einsatzes von CGI an Filme herangehen, sondern auch in „beide Richtungen“ CGI und reale locations miteinander verwechseln: So wie fotorealistische CGI-Landschaften für reale locations gehalten werden können, werden auch umgekehrt reale Landschaften für CGI gehalten. Diese perzeptivische Verwirrung wird durch „erklärende“ Meta- und Paratexte wie Makings Ofs nicht unbedingt verringert, sondern kann diese sogar verstärken, denn die Tatsache, dass viele Zuschauer die genannten (realen) Berge für „pure CGI“ 
hielten, lässt sich gerade auf das von Para-/Metatexten geprägte Vorwissen um den immensen Einsatz von CGI in diesen Filmen zurückführen.

Aber auch wenn Zuschauer tatsächlich die CGI-Landschaften als solche „entlarven“, so beruht eine Kritik daran in erster Linie auf einer in den Augen der Zuschauer mangelhaften Umsetzung der digitalen Effekte und nicht auf einem „Zuviel“ an digitalen Effekten. Gerade die Erkennbarkeit der Digitalität wird kritisiert und nicht der „exzessive“ Einsatz von CGI. Es wird demnach die Qualität und weniger die Quantität der CGI kritisiert.

Trotz des „Zugeständnisses“ Peter Jacksons an die neuseeländische Nation, auch im Hobbit wieder neuseeländische locations einzusetzen, ist dennoch klar erkennbar, dass sich in seinen Filmen der CGI-Anteil immer weiter erhöht und der Anteil realer locations immer weiter abnimmt. Ironischerweise treibt damit gerade der oft als neuseeländischer „Nationalheld“ bezeichnete Sir Peter Jackson die Entlokalisierung der neuseeländischen location entscheidend voran, was ihm bereits mehrfach Kritik bei Zuschauern und Kritikern eingebracht hat. Jacksons Fokus hat sich somit von Filmen mit (oftmals humoristischer) Gesellschaftskritik (Bad Taste, Braindead, Heavenly Creatures) über landschaftsfokussierte Filme (Lord of the Rings-Trilogie), hin zu „technikdominierten“ Filmen (Hobbit-Trilogie, King Kong) entwickelt. Den vorläufigen Höhepunkt dieser Entwicklung hat Peter Jackson mit dem als nächstes zu analysierenden Film King Kong erreicht, einem Film, bei dem praktisch keine realen locations mehr eingesetzt worden sind und der damit der 7. Simulationsstufe zuzuordnen ist.

\subsubsection{King Kong (2005)}

Bei Peter Jacksons King Kong handelt es sich im Gegensatz zu den bisher analysierten Filmen um einen Film der 7. Simulationsstufe. Während die Hobbit-Trilogie bereits einen hohen Anteil digitaler Landschaften aufbot, kommt King Kong quasi ohne locations aus, wie Production Designer Grant Major anführt: „Everything shot on this movie, bar one or two minor instances, has been studio-based [...] there are no locations, other than the Opera House and Civic Theater" (Wake 20IO: 3I). Diese Rückkehr zu alten Hollywood-Studio-Traditionen geht einher mit einem gleichzeitigen Sprung nach vorne in puncto CGI: mit über 2500 effect shots und damit sowohl die eigenen Lord of the Rings-Filme als auch Star Wars Episode III (2005) übertreffend, wird King Kong 
als Meilenstein in der Geschichte der Visual Effects angesehen. ${ }^{86}$ Letztlich handelt es sich bei King Kong um einen Hybriden aus 6. und 7. Simulationsstufe: Zum einen ist der digital generierte Handlungsort New York zwar nicht im realen New York gedreht worden, aber dennoch mit dem realen New York assoziierbar (6. Simulationsstufe), zum anderen ist Skull Island ebenfalls digital generiert, darüber hinaus aber auch fiktiv und somit nicht ohne weiteres mit einer realen Geografie assoziierbar (7. Simulationsstufe) ${ }^{87}$

Wieso aber kamen für die Darstellung Skull Islands keine neuseeländischen Landschaften mehr zum Einsatz? Immerhin hatte Neuseeland seine Tauglichkeit zur Simulation vormoderner Welten in der Vergangenheit vielfach unter Beweis gestellt? Nicht nur Fantasywelten, sondern auch archaische Regionen, wo sich die Aufzucht von Dinosauriern „anbot“ (The Lost World: Jurassic Park [1997]) wurden (auch) auf Neuseeland gedreht. Auf Skull Island treiben neben dem Riesengorilla ebenfalls Dinosaurier ihr Unwesen, sodass die vielerorts prähistorisch wirkende Landschaft Neuseelands damit gut harmoniert hätte. Der Grund, warum dennoch weder neuseeländische noch irgendwelche anderen realen locations mehr zum Einsatz kamen, lässt sich in den mittlerweile weit fortgeschrittenen Möglichkeiten digitaler Landschaftsgenerierung finden. Offenbar genügten neuseeländische locations Peter Jacksons Anforderungen an die Hypersublimität und Fantastik von Skull Island nicht, sodass er auf die nahezu unbegrenzten Darstellungsmöglichkeiten von CGI (in Kombination mit Miniaturmodellen) zurückgriff. So äußert sich Jackson im Hinblick auf die Unterschiede zwischen dem Original von 1933 und seinem Remake: „What I want to do is see what would happen if Willis O’Brien, the guy who did this stuff in 1933 , had at that time the gadgets that we have today. And he also said, ,I'd like people to say, ,I knew New Zealand had some fantastic locations, but, wow, where did you find Skull Island?" (Wake 20I0: 3I). Jackson wollte folglich durch CGI eine „Steigerung“ zu den ohnehin schon „fantastischen“ neuseeländischen locations erreichen. Diese Steigerungsabsicht ist ein Beleg für den im Methodikteil besprochenen „Zwang zur Gigantomanie“, wie Hoberg es nennt (1999: 218), wodurch ein „anästhetisiertes“ Publikum immer von Neuem sensorisch überwältigt und zum Staunen angeregt werden soll, was wiederum den kommerziellen Erfolg solcher Filme garantieren soll.

Die Filmemacher strebten bei der Konstruktion von Skull Island perzeptiven Realismus an, gleichzeitig sollte der Film aber auch eine ästhetische Hommage an das Original darstellen. Durch

\footnotetext{
${ }^{86}$ So z. B. auf www.filmsite.org/visualeffects2o.html, wo King Kong unter die „Greatest Visual and Special Effects (F/X) - Milestones in Film 2003-2005" eingeordnet wird.

${ }^{87}$ Es gibt aber durchaus Sonderfälle, in denen selbst eine digital generierte, fiktive Landschaft mit einer realen Geografie assoziiert werden kann, wie bei der Analyse von Avatar demonstriert werden wird.
} 
diesen intertextuellen Bezug hat die Landschaft einen leicht „malerischen“ Charakter, den Peter Jackson an Gustave Dorés expressionistischen Stil angelehnt hat. Jackson argumentiert, dass der Einsatz realer locations hierbei für seine kreative Vision sogar hinderlich gewesen wäre:

$[\ldots]$ the 1933 jungles are very stylized, very much based on Gustave Doré's artwork. Things close to you are silhouetted and dark, and as they go further away they get progressively flatter and brighter and foggier. I just couldn't imagine doing King Kong without somehow doing that, and it would be impossible to do it on location. You couldn't control the elements. That look is artificial, there's a certain art-directed style to it, and it has to be done in a very technically controllable way (Wake 20Io: 3I).

Deshalb wurde nicht nur aus pragmatischen, sondern auch aus kreativen Gründen CGI eingesetzt, das nach Ansicht der Filmemacher im Gegensatz zu locations kein Kreativitätshindernis darstellt. Trotz der Stilisierung sollte die Welt aber dennoch „real“ wirken und maximal immersiv sein. Um dies zu bewerkstelligen behandelte Jackson Skull Island nicht als eine Fiktion, sondern als real existierenden Ort, wie Director of Photography Alex Funke anmerkt: „[...] this is no fantasy. As far as Peter is concerned, Skull Island is a real place [...]“ (Wake 20IO: I40). Somit verfolgt Jackson eine ähnliche Authentisierungsstrategie wie schon bei Lord of the Rings, wo er der Filmcrew gegenüber ebenfalls die Anweisung gab, Mittelerde wie eine reale, historische Welt (ähnlich wie Troja) zu behandeln. Dieser Authentizitätseffekt sollte durch Paratexte wie dem pseudohistorischen Buch The World of Kong. A Natural History of Skull Island weiter verstärkt werden. Hierbei wird für King Kong-Fans, die noch tiefer in die Welt von Skull Island eintauchen wollen, eine „Historie“ der Insel konstruiert.

Ironischerweise ist das filmische Skull Island aber letztendlich außerordentlich irreal, da im Gegensatz zu Lord of the Rings eben keine realen locations mehr eingesetzt wurden, die ein Filmtourist besuchen könnte. Insofern ist es wenig verwunderlich, dass King Kong keinen Filmtourismus nach Neuseeland ausgelöst hat, ${ }^{88}$ obwohl aus vielen Rezensionen eine starke Faszination für Skull Island ersichtlich wird und die Filmwelt trotz ihrer Digitalität als authentisch erachtet wird, wie die Rezension von „dunmore_ego“ illustriert:

Once upon Skull Island, the movie ratchets into the stratosphere, in sequences where we realize that, due to his pedigree, Jackson is the only person who could have remade *King Kong* with any semblance of majestic authenticity. In fact, the whole Skull Island interlude

\footnotetext{
${ }^{88}$ Ob King Kong Filmtourismus nach New York ausgelöst hat, konnte im Rahmen dieser Arbeit nicht festgestellt werden.
} 
is so rife with arterially-bursting CGI that "breathtaking" or "awe-inspiring" are not only

understatements, they are sins against humanity (27. December 2005).

Dem pathetischen Sprachstil des Rezensenten nach zu urteilen ist sowohl die Authentisierungsstrategie als auch das Ziel der Filmemacher, Zuschauer durch hypersublime CGI sensorisch zu überwältigen, zumindest für einen Teil der Zuschauerschaft gelungen und belegt eindrücklich, dass ein großer Teil der Zuschauer den Einsatz realer locations nicht als unbedingt notwendig erachtet. Zweifelsohne gibt es auch negative Rezensionen wie die von „jholmstrom-I“, wo eine „orgy of CGI-effects with no end in sight“ (25. Dezember 2005) beklagt wird und eine suspension of disbelief als nicht möglich erachtet wird. Allerdings zeigt der kommerzielle Erfolg des Films und die gute Bewertung in Internetforen wie IMDb und Rottentomatoes, dass die große Mehrheit der Zuschauer CGI durchaus als authentisch akzeptieren kann.

Aufgrund der fehlenden locations hat King Kong trotz der Begeisterung vieler Zuschauer für Skull Island keinen Filmtourismus ausgelöst, wobei Jackson im Jahr 20 Io in den Universal Studios eine „King Kong 360 3-D“-Tour einrichten ließ, nachdem die frühere King Kong-Attraktion 2008 bei einem Brand zerstört wurde (Goldberg, 2010). Hierbei handelt es sich aber um Filmstudio-Tourismus, der beinahe ebenso virtuell ist wie das Filmerlebnis selbst, da die Besucher nicht mehr wie noch bei der abgebrannten Attraktion eine physische King Kong-Figur zu sehen bekommen, sondern stattdessen einen knapp 3minütigen 3 D-Film sehen, während sie in einem Gefährt sitzen. Hierbei handelt es sich folglich um eine ganz andere Art von Filmtourismus als beim Lord of the Rings-Tourismus in Neuseeland. In besonderen Fällen kann jedoch auch ein Film der 7. Simulationsstufe Filmtourismus auslösen, wie der als nächstes zu analysierende Film Avatar zeigen wird.

\subsubsection{Avatar (2009)}

Im Gegensatz zu King Kong, wo durch die Darstellung eines digitalen New Yorks immer noch ein Bezug zu einem realen Ort gegeben ist, spielt sich das Geschehen in Avatar gänzlich in einer fiktiven, digital generierten Welt ab. Somit ist der von James Cameron gedrehte und unter Mitarbeit von Jacksons WETA-Studios entstandene Film Avatar nicht mehr wie King Kong ein Hybride, sondern ein „reiner“ Film der 7. Simulationsstufe. Ebenso wie King Kong gilt Avatar als Meilenstein in puncto CGI und erhielt hierfür auch den Oscar für beste visuelle Effekte. 
Tatsächlich hat gerade Peter Jacksons King Kong James Cameron davon überzeugt, dass die CGI-Technologie mittlerweile so weit fortgeschritten ist, dass es an der Zeit sei, das lange geplante Filmprojekt Avatar in die Tat umsetzen (Cameron, 2009). Ähnlich wie bei King Kong kamen bei Avatar keine realen locations mehr zum Einsatz, weil den Zuschauern etwas nie zuvor Gesehenes geboten werden sollte und sich dies am besten bewerkstelligen ließ, indem Pandora, die Welt von Avatar, von Grund auf neu konzipiert wurde, statt sich durch den Einsatz von locations in den kreativen Möglichkeiten von vornherein einzuschränken. Trotz der Fantastik der Landschaft sollte diese aber dennoch so real wie möglich auf die Zuschauer wirken, wozu größtmöglicher Fotorealismus - quasi als Surrogat für die fehlenden locations - angestrebt und zusätzlich in $3 \mathrm{D}$ gedreht wurde, um die Immersivität weiter zu steigern. Hierdurch sollten die Zuschauer den Film letztendlich als „window into the world of Pandora“ betrachten können (Duncan/Fitzpatrick 20IO: 223, 25I). Insbesondere die im Film als „Hallelujah Mountains“ bezeichneten fliegenden Berge illustrieren diese Kombination von Hypersublimität und gleichzeitigem perzeptiven Realismus eindrücklich:

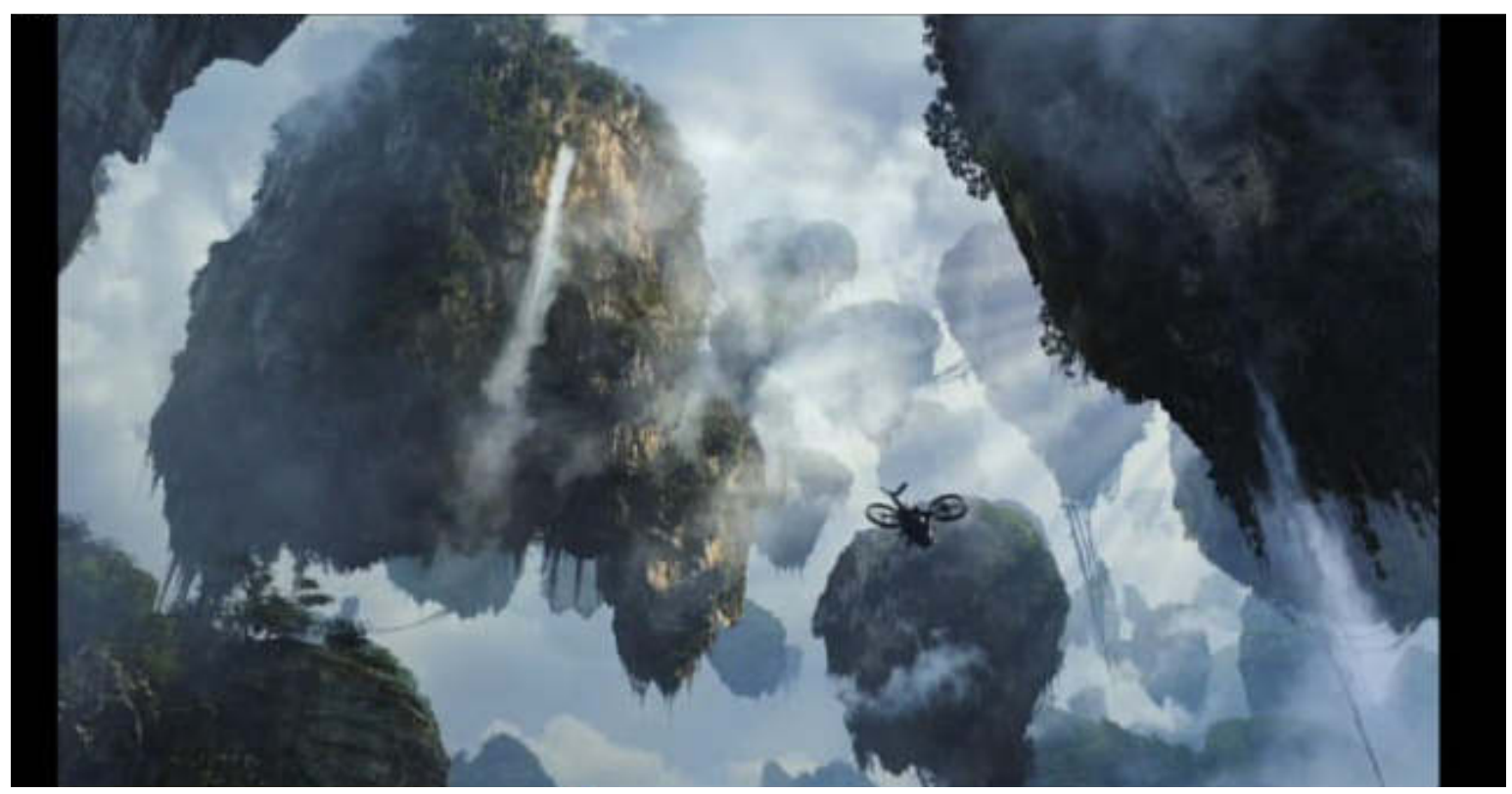

Abbildung 3.21: Hallelujah Mountains [0o:53:44]

Auch wenn die fliegenden Berge (siehe Abbildung) offensichtlich irdische physikalische Gesetzmäßigkeiten sprengen, soll durch möglichst realistische Darstellung der Lichtreflexionen, einer natürlich wirkenden Irregularität der Gesteinsformationen und die freien, die Dreidimensionalität beto- 
nenden Kamerafahrten die Landschaft von den Zuschauern als potenziell „real“, d. h. als possible world akzeptiert werden.

Die vollständig digitale, „authentische“ Simulation einer Welt, die sich in vielerlei Hinsicht physikalisch wie die Welt verhält, die wir kennen, erforderte einen immensen Rechenaufwand, da selbst kleinste Details der „analogen“ Welt in das digitale Format transferiert werden mussten, wie Cameron anführt: „So many things that the eye and brain take for granted in real-world experience must be minutely studied, understood and deconstructed, and ultimately reverse-engineered in the sterile, mathematical pseudo-reality of the computer" (Duncan/Fitzpatrick 2010: 252). Das Streben nach maximaler Glaubwürdigkeit ging letztlich so weit, dass mittels der Software Massive ein digitales, selbstregulierendes „Ökosystem“ erschaffen wurde: „When artists planted seeds on a terrain, Massive would simulate a forest growing and competing for light and space. Bigger trees grew quickly, smaller plants died, and shade-loving ferns grew around the base of the large trees" (Robertson, 2009).

Zwar gab es wie schon bei King Kong zweifelsohne auch negative Zuschauerrezensionen, allerdings zeigt sich auch hier wieder, dass die Kritik oftmals auf einem Vorwissen um den Einsatz von CGI herrührt und mehr der CGI-Einsatz an sich bemängelt wird und weniger von einem tatsächlichen „Erkennen“ der „unrealistischen“ CGI herrührt. Beispielsweise schreibt der User „uncreativeperson7“ in einer Forendiskussion über die visuellen Effekte in Avatar: „That's the key to successful CGI: There needs to be something real in the frame to tie everything together" (I2. Februar 20I5). Kritikwürdig ist für diesen User der Einsatz „purer“ CGI ohne den Einsatz realer locations oder Objekte. Fakt ist aber, dass durch den Einsatz sogenannter Fototexturen, ${ }^{89}$ d. h. digital fotografierten Fragmenten realer Landschaften diese „Spur des Realen“, die der User fordert, auch in Avatar vorhanden ist und daher von „reiner“ CGI nicht die Rede sein kann. Insofern zeigt sich auch hier eine pauschale Ablehnung von CGI als „unrealistisch“, ohne aber sicher zwischen CGI und real abgefilmten Objekten differenzieren zu können..$^{90}$

In Gesamtbetrachtung scheint sich der Aufwand der Filmemacher aber gelohnt zu haben, da ein Großteil der Zuschauer keineswegs mangelnden perzeptiven Realismus beklagt, sondern

\footnotetext{
${ }^{89}$ Auf die Fototexturen wird im Verlauf der Analyse noch genauer eingegangen werden.

${ }^{9 \circ}$ Es ist davon auszugehen, dass ein großer Teil dieser Zuschauer den Film als wesentlich „realistischer“ einstufen würde, wenn ihnen in Making Ofs und anderen Paratexten die (falsche) Information, dass reale locations eingesetzt worden wären, vermittelt worden wäre.
} 
Avatar im Gegenteil als äußerst immersiv empfindet. ${ }^{9 \mathrm{I}}$ Mitunter wird sogar die CGI-Landschaft in Avatar im Vergleich zu den im compositing-Verfahren konstruierten Landschaften in Lord of the Rings als die authentischere Fantasy-Landschaft betrachtet. So schreibt „blackmage439“: „Peter Jackson did a fantastic job of bringing those thoughts and words to life. The epic adventure of ,Lord of the Rings' was great, but it was always lacking in the world it created. It never felt far, far away or different, especially if one already knew most exterior shots were performed in New zealand. Cameron took this idea of a fantastic, alien world and literally brought it to the stars“ (II. Februar 20I0). Hier wird demnach CGI-generierte Landschaft im Vergleich zu location-Drehs als die „authentischere“ Form eingestuft, wenn es um die Darstellung fantastischer Welten geht, da location-Drehs trotz des Einsatzes von CGI noch zuviele Charakteristika des „Irdischen“ enthalten, die aber nach Ansicht solcher Zuschauer die Glaubwürdigkeit fantastischer Welten verringert. Dies illustriert, wie weit Zuschauervorstellungen von einer ,authentischen“ Fantasywelt auseinandergehen können: Für einen Teil der Zuschauer ist der Einsatz realer locations wichtig, für andere stellt gerade der location-Einsatz ein Manko in der Darstellung fiktiver Welten dar.

Viele Zuschauer beteuern überdies, dass Pandora wie eine reale location aussehe, was einmal mehr illustriert, dass Zuschauer CGI-Landschaften mittlerweile durchaus als adäquaten Ersatz für reale locations betrachten. Auch gehen etliche Zuschauer irrtümlich davon aus, dass der Film nicht nur in Neuseeland produziert, sondern auch dort on location gedreht worden sei, da ihnen aufgrund der perzeptiv realistischen Darstellung nicht in den Sinn kommt, dass die Landschaft komplett virtuell erzeugt worden sein könnte. Hierzu kommentiert James Cameron in einem Interview: „Viele Leute glaubten, dass wir in Neuseeland gedreht haben, weil es dort Regenwald gibt. Klar waren wir im Regenwald - zwischendurch, zur Erholung. Gedreht haben wir im Studio. Sicher liegt eine gewisse Ironie darin, dass wir die Natur in "Avatar" feiern, indem wir eine ganz und gar künstliche Natur am Computer erschaffen“ (Cameron, 2009). Die Ironie, die Cameron darin sieht, dass Natürlichkeit in Avatar auf vollständig künstliche Weise dargestellt wird, stellt eine Parallele zur digitalen Simulation „historischer“ Landschaften in Lord of the Rings und The Hobbit dar. In beiden Fällen liegt das Paradoxon darin, dass etwas Vormodernes (Natur, historische Artefakte) durch den Einsatz postmoderner Technologie fast adäquater dargestellt werden kann als durch die entsprechenden realen Objekte selbst.

\footnotetext{
${ }^{91}$ Ein großer Teil der negativen Rezensionen bemängelt außerdem in erster Linie den „unoriginellen“ Plot, lobt aber gleichzeitig das visuelle Spektakel.
} 
Neben den Forendiskussionen über den perzeptiven Realismus des Films bringen viele Zuschauer in ihren Rezensionen ihre Begeisterung und Sehnsucht nach der fiktiven Welt Pandora zum Ausdruck, was eine wichtige Voraussetzung für film tourism inducement darstellt. Trotz der Darstellung einer fiktiven Welt, die ohne den Einsatz realer locations auskommt, hat Avatar erstaulicherweise Filmtourismus ausgelöst - allerdings nicht nach Neuseeland, sondern nach China. Wie aber kam es dazu, was veranlasste Zuschauer, China als einen „Referenten“ von Pandora zu behandeln, so wie zuvor Zuschauer Neuseeland als „Referenten“ von Mittelerde behandelt hatten? Im Gegensatz zu Lord of the Rings wurde Avatar ja nicht on location (also auch nicht in China) gedreht?

Die Antwort hierauf lässt sich in dem Bestreben der Filmemacher nach maximalem Fotorealismus finden: Zwar wurde auf on location-Drehs verzichtet, es kamen aber sogenannte Fototexturen von chinesischen, südamerikanischen, hawaiianischen und neuseeländischen Landschaften zum Einsatz (Duncan/Fitzpatrick 2010: 57, II3). Hierbei wurden die digital generierten Landschaften gewissermaßen mit digitalen Fotografien real existierender Landschaftsobjekte aus den genannten Ländern (perspektivisch korrekt) „,beklebt“.92 So wurden beispielsweise die erwähnten Hallelujah Mountains unter Zuhilfenahme von Fototexturen aus der chinesischen Guilin-Region konstruiert. Denn trotz der immensen Fortschritte in der CGI-Technologie sind Filmemacher der Ansicht, dass für maximalen Fotorealismus immer noch Referenzpunkte aus der Realität in der Landschafts- und Figurendarstellung vonnöten sind. Insofern ist also auch bei Filmen der 7. Simulationsstufe meist noch ein letzter Rest an Indexikalität und - abhängig von Art und Umfang der Fototexturen - auch Ikonizität gegeben. Duncan betont in seinem Buch The Making of Avatar, dass diese Texturen für Cameron nur als Ausgangspunkt gedient hätten, um etwas Neues zu schaffen und nicht etwa gleichbedeutend seien mit locations als „Designvorlagen“: „[...] although these natural settings and others served as a touchstone, Cameron encouraged the artists to create utterly original environments, to make Pandora a world unlike any seen before (Duncan/ Fitzpatrick 20I0: 57).

Wohlgemerkt hat Avatar nur Filmtourismus nach China ausgelöst, nicht aber nach Neuseeland, Südamerika oder Hawaii, was zum einen auf den unterschiedlichen Umfang der eingesetzten Fototexturen zurückzuführen ist, zum anderen auf die explizite Selbstvermarktung der chinesischen Zhangjiajie- und Huangshan-Nationalparks als das „reale Pandora“. Wie die Daily Mail

\footnotetext{
${ }^{92}$ Hierzu schreibt Manovich: ,[...] instead of defining something from scratch through the algorithms, they can simply sample it from existing reality and incorporate these samples in the construction process (2001: 30).
} 
berichtet, haben beide Nationalparks einen Besucherzuwachs von rund 8o \% seit dem Erscheinen von Avatar verbuchen können und konkurrieren seitdem miteinander, wer nun die primäre Inspirations- und „Fototexturenquelle“ für Pandora gewesen sei („Prove Another Park Inspired Avatar“, 20I2). Auch wenn China die Selbstvermarktungsstrategie Neuseelands zu kopieren versucht, gibt es einige wichtige Unterschiede: Während sich mit Neuseeland eine ganze Nation als „Mittelerde“ ausgibt, ist „Pandora“ auf zwei verhältnismäßig kleine Regionen in China begrenzt und dürfte daher nur einen relativ begrenzten Einfluss auf das nationale Selbstbild Chinas haben. Die größere Distanz und „Nüchternheit“ in der Selbstvermarktung lässt sich auch an dem Slogan „Pandora is far but Zhangjiajie is near" erkennen, was weniger extrem anmutet als Neuseelands Slogan „Welcome to Middle-Earth“, wo quasi suggeriert werden soll, dass Neuseeland tatsächlich die physische Realisierung einer fiktiven Welt sei.

In der von Zhangjiajietourism angebotenen I5-tägigen Tour „Fairyland Jiuzhaigou \& Avatar Mountains ${ }^{\text {(993 }}$ ist in der Reisebeschreibung kein einziges Mal von konkreten Avatarlocations die Rede, was verständlich ist, weil es ja gar keine „locations“ für Avatar gibt. Stattdessen werden Aktivitäten wie Panda-Besichtigungen, Opernshows und Klosterbesuche angeboten, die keinerlei Bezug zum Film haben. Tatsächlich ist es fraglich, ob solch eine Tour „Hardcore“-AvatarFans zufriedenstellen kann, da neben einer „abstrakten“ Ähnlichkeit zwischen Zhangjiajie und Pandora letztlich nur nicht-filmrelevante Aktivitäten angeboten werden, was in deutlichem Kontrast zu neuseeländischen Lord of the Rings-Tour-Anbietern steht, wo nahezu ausschließlich Lord of the Rings-locations besucht und filmrelevante Aktivitäten wie das Nachstellen von Filmszenen und dem Genießen eines Frühstücks mit „Lembas-Brot“ (dem Brot der Elben) angeboten werden (Leotta 20II: 168). ${ }^{94}$

Wenn man das in Abbildung 3.22 zu sehende Karstgebirge des Zhangjiajie-Nationalparks mit den filmischen Hallelujah-Mountains (Abbildung 3.21, siehe oben) vergleicht, lässt sich nur eine geringe Ähnlichkeit zwischen beiden Landschaften feststellen.

\footnotetext{
${ }^{93}$ www.zhangjiajietourism.us/packages-chinaTour.html

${ }^{94}$ Leider liegen keine Daten zur Zufriedenheit der Avatar-Filmtouristen vor. Eine Feldstudie und ein daran anschließender Vergleich mit den Daten, die beispielsweise Roesch und Carl zum Lord of the Rings-Filmtourismus gesammelt haben, wäre in jedem Fall interessant und aufschlussreich.
} 


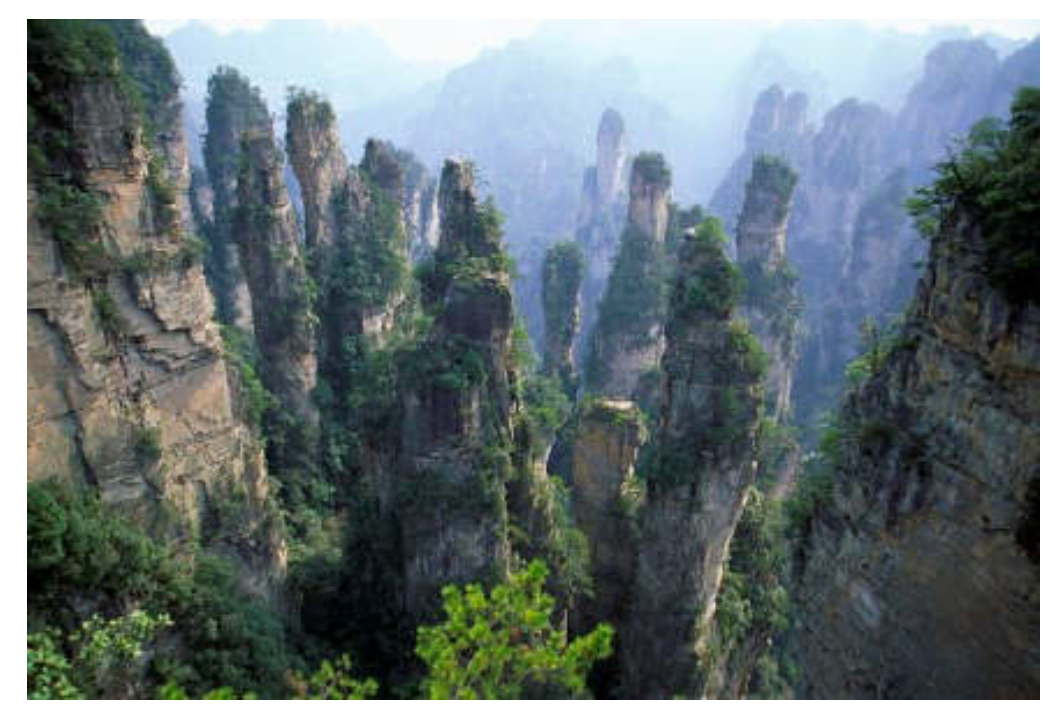

Abbildung 3.22: Karstgebirge des Zhangjiajie-Nationalparks ${ }^{95}$

Während bei Lord of the Rings und The Hobbit aufgrund der eingesetzten locations immer noch häufig die Möglichkeit bestand, anhand relativ deutlich zu erkennender Ähnlichkeit in der Geomorphologie zwischen Handlungsort und location bei Filmtouristen einen Wiedererkennungseffekt auszulösen, ist die Ähnlichkeit zwischen den Hallelujah Mountains und Zhangiajie doch recht abstrakt, da Zhangjiajie eben nur als Quelle für Fototexturen diente, die aber in einem völlig anderen landschaftlichen Kontext (schwebende Berge usw.) eingesetzt wurden. Dieser geringe Grad an Ikonizität hat dennoch ausgereicht, um Filmtourismus auszulösen, was illustriert, dass die Intensität der Zuschauersehnsucht, eine fiktive Welt „in der Realität“ zu erleben, wichtiger ist als die tatsächliche Ähnlichkeit zwischen fiktiver Welt und realer location bzw. „Fototexturenquelle“.

Ein Mindestmaß an Ikonizität muss aber in jedem Fall gegeben sein: Wenn in puncto Ikonizität eine kritische Schwelle unterschritten wird, entsteht trotz starker Zuschauersehnsucht dennoch kein Filmtourismus, weil das entsprechende Land, dass als „Fototexturenquelle“ gedient hat, keine plausible Grundlage mehr für eine filmtouristische Selbstvermarktung hat. So ist trotz der eingesetzten Fototexturen und der Hinweise auf der Tourism New Zealand-Website, dass Avatar in Neuseeland gedreht worden sei, Neuseeland von Filmtouristen im Gegensatz zu China nicht als Teil der physischen „Realisierung“ Pandoras akzeptiert worden. Dass Neuseeland nicht von einem Avatar-Filmtourismus profitiert hat, liegt daran, dass die Ähnlichkeit zwischen Neuseeland und Pandora zu gering und der Ikonizitätsgrad somit nicht ausreichend ist. So zeigt folgende

\footnotetext{
${ }^{95}$ Bildquelle: www.dailymail.co.uk/news/article-2182690/Chinese-national-park-offers-IO-০oo-challenge-disprove-claiminspiration-Avatar.html (Zugriff: I. Juli 20I5), Copyright: Daily Mail
} 
Abbildung den Einsatz neuseeländischer Fototexturen in Form des koru, dem für neuseeländische Wälder charakteristischen Silberfarns in seinem eingerolltem Frühstadium.
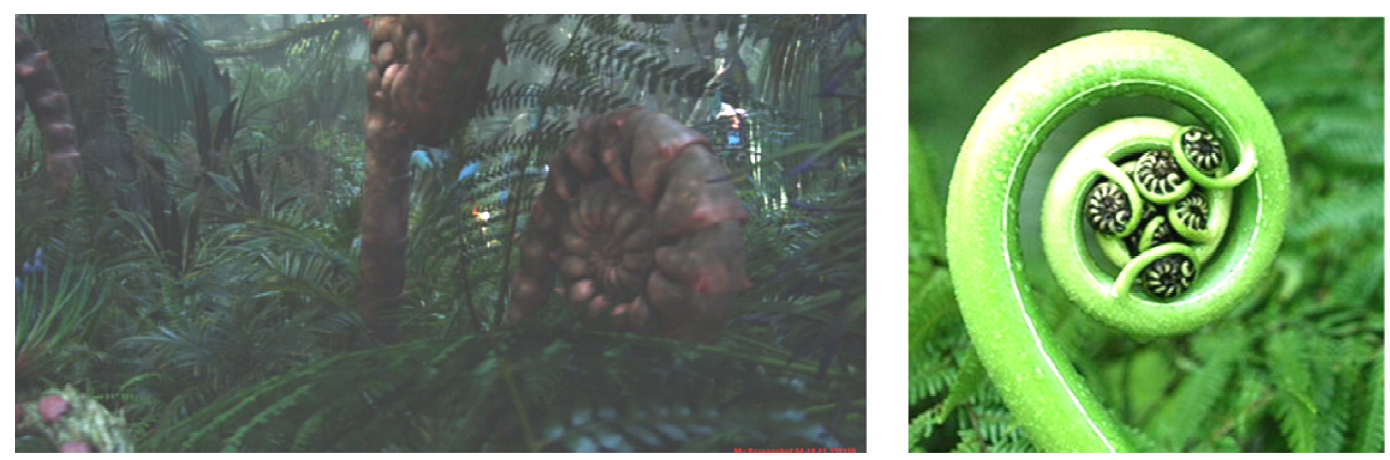

Abbildung 3.23: Links gigantische und rot gefärbte koru in Avatar [00:24:45], rechts der neuseeländische koru ${ }^{96}$

Obwohl der Silberfarn ein Nationalsymbol Neuseelands ist und somit auch Touristen familiär vorkommen dürfte, reicht dieses landschaftliche Detail der Avatar-Welt nicht aus, um als Zuschauer Neuseeland mit Pandora assoziieren zu können.

Auffällig ist, dass die Begeisterung für die Welt von Avatar trotz - oder gerade wegen - des sehr geringen Bezuges zur realen Welt ausgesprochen hoch ist. So schreibt Rezensent „Daniel Pall Jonasson“: „I feel like I became part of this world and now, at home, I kind of miss the scenery, the animals, the people and the nature of the planet“ (20. Dezember 2009). Während einige Zuschauer ihre starke Sehnsucht nach Pandora durch Besuchen der chinesischen Nationalparks befriedigen können, scheint dies bei anderen Zuschauern nicht der Fall zu sein. So schreibt der User Ivar Hill: „One can say my depression was twofold: I was depressed because I really wanted to live in Pandora, which seemed like such a perfect place, but I was also depressed and disgusted with the sight of our world, what we have done to Earth. I so much wanted to escape reality“ (Piazza, 20Io). Da diese Sehnsucht bei einigen Zuschauern sogar derart stark ist, dass sie in Foren suizidale Absichten äußern, weil sie ihre Sehnsucht nach der rein virtuellen Welt Pandoras nicht realisieren können (Thomas, 20Io), wird dieses Phänomen als „Post Avatar Depression-Syndrome“ (PADS) bezeichnet, was bei den bisher analysierten Filmen in dem Ausmaß nicht zu beobachten war. So ist beispielsweise trotz der vielen Fans kaum von einem „Post-Lord of the Rings-Syndrome“ die Rede. Zwar ist PADS kein wissenschaftlicher Terminus und hat zudem einen leicht ironischen Unterton,

\footnotetext{
${ }^{96}$ Bildquelle (gespiegelt): https://www.korucomputing.com/(Zugriff: 3. Juli 20I5), Copyright: Korucomputing
} 
dennoch werden die in den Foren geäußerten psychischen Probleme einiger User von Psychologen durchaus ernstgenommen (Mette, 2010).

Zwar lässt sich ein Teil der PADS-Problematik durch die von Psychologen hervorgebrachte Vermutung erklären, dass diejenigen Zuschauer, die depressiv werden, bereits vor dem Betrachten des Films psychische Probleme hatten (Mette, 20I0). Ein Teil der Problematik ist aber meines Ermessens auch auf der überindividuellen gesellschaftlichen Ebene zu suchen. Die Filmemacher Avatars scheinen in ihrem „Zwang zur Gigantomanie“ (Hoberg 1999: 218) eine von vielen Zuschauern als derart immersiv und fantastisch erachtete Welt geschaffen zu haben, dass sie als perfekte Simulation erachtet wird und ein geradezu pathologisches Verlangen, die Simulation zur Realität werden zu lassen, auslöst. Der Zwang zur Gigantomanie rührt aber daher, dass Zuschauer eben diese ständige Reizsteigerung aktiv einfordern, was auch daran ersichtlich wird, dass sich eine deutliche Korrelation zwischen CGI-Lastigkeit und Umsatz feststellen lässt: Avatar, Lord of the Rings und Harry Potter gehören zu den weltweit (kommerziell) erfolgreichsten Filmen überhaupt und zeichnen sich allesamt durch innovative digitale Effekte aus.

Wenn Baudrillard eine Art „Tod des Realen“ konstatiert, so scheinen viele AvatarZuschauer tatsächlich das Verlangen zu haben, ihre Realität zu „töten“ und in die Simulation zu flüchten. In einer „Gesellschaft der Simulation“ spielt CGI eine Schlüsselrolle, da erst durch digitale Effekte eine derart immersive und zugleich hypersublime Welt konstruiert werden kann, dass diese mit keiner in der realen Welt vorzufindenden Landschaft oder Region mehr vergleichbar ist. Ähnlich einem Drogenrausch scheint diesen Zuschauern durch das Betrachten fantastischer Scheinwelten der Alltag erst recht unerträglich grau zu sein, und es wird ein unrealistisches, unerfüllbares Landschaftsideal geschaffen. Hinzu kommt, dass es sich bei Avatar um einen Film der 7. Simulationsstufe handelt, sodass für diese Zuschauer trotz des Einsatzes von Fototexturen der Bezug zur realen Welt nicht mehr ausreichend ist, um Pandora noch in der realen Welt verorten zu können, so wie es die Lord of the Rings-Touristen noch konnten. Abschließend lässt sich mit Hoberg feststellen: „In der ,Gesellschaft des Spektakels‘ (Debord) füllen Bilder scheinhaft reale Defizite aus und avancieren zu der idealen Ware schlechthin" (1999: 215).

\subsubsection{Ergebnis}

Die Analyse der Filme der 5. und 7. Simulationsstufe hat demonstriert, dass bei Fantasyfilmen neuseeländische locations zunehmend durch digitale Landschaftselemente maskiert und 
schließlich vollständig ersetzt werden, was bedeutet, dass der Trend dahin geht, dass die 7. Simulationsstufe die 5. Simulationsstufe verdrängt. Es hat sich gezeigt, dass die Filmemacher der hier analysierten Filme der Ansicht sind, dass mit CGI mittlerweile Fantasywelten ebenso überzeugend oder sogar noch überzeugender in ihrer (Hyper-)Sublimität, Archaik und ihrem Fotorealismus dargestellt werden können als mit locations. Es sind somit nicht nur pragmatische Gründe wie maximale Produktions- und Kostenkontrolle (kein störendes Wetter, Drehverbote, störende Landschaftsartefakte), sondern auch kreative und ästhetische Gründe, die den Umstieg auf immer mehr Digitalität begründen - wobei locations teilweise sogar als explizites Kreativitätshindernis erachtet werden, während digitale Landschaften in den Augen der Filmemacher ihrer kreativen Vision deutlich näher kommen können. Das bisherige Manko von CGI, nämlich mangelnder perzeptiver Realismus, scheint weitgehend überwunden zu sein. Damit büßen locations aber ihren Hauptvorzug des „naturgemäßen Fotorealismus“ gegenüber der CGI ein, was auch für neuseeländische locations gilt, die als besonders „authentisierende“ Projektionsflächen für Fantasywelten bekannt geworden sind.

Die ständige Reizsteigerung, die Zuschauer gerade bei Fantasyfilmen erwarten, d.h. die Darstellung immer fantastischer und irrealer werdender Fantasywelten kann nur mittels CGI realisiert werden. Es gilt letztendlich: Je hypersublimer die Fantasywelt erscheinen soll, desto mehr CGI und desto weniger location wird eingesetzt. Parallel zu der Reizsteigerung erwarten Zuschauer aber weiterhin perzeptiven Realismus; für einen Großteil der Zuschauer macht gerade die Suggerierung einer „Realität des Irrealen“ den Reiz dieser Filme aus. Bei aller Fantastik soll die Filmwelt „echt“ wirken, da ansonsten eine suspension of disbelief erschwert wird und beispielsweise eine „verpixelte“, d.h. perzeptiv völlig unrealistische Fantasywelt von Zuschauern nicht als possible world akzeptiert wird. Hierbei ist ein „irdisches“ Aussehen der Fantasylandschaft keine Voraussetzung für deren Glaubwürdigkeit, vielmehr wird gerade das zu „irdische“ Aussehen von Fantasywelten, die unter Zuhilfenahme von locations konstruiert wurden, oft als nicht „fantastisch genug“ kritisiert.

$\mathrm{Da}$ „Authentizität“ eine subjektive Kategorie ist, gibt es zweifelsohne auch Zuschauer, die locations ohne CGI als einzig authentische Projektionsfläche für Fantasyfilme akzeptieren und dies in ihren Rezensionen auch zum Ausdruck bringen. Da aufgrund des Fotorealismus der CGI eine Differenzierung zwischen digitalen Landschaften und locations aber gar nicht mehr ohne weiteres möglich ist, scheint die Forderung nach on location-Drehs mehr ein nostalgisches Bedürfnis dieser 
Zuschauer zu sein und weniger ein tatsächliches „Erkennen“ „inauthentischer“ digitaler Landschaften.

Dass sowohl die Filmemacher als auch ein großer Teil der Zuschauer digitale Landschaften als adäquaten Ersatz für reale locations erachten, hat auch Auswirkungen auf den Filmtourismus: Je mehr CGI eingesetzt wird, desto weniger Ähnlichkeit besteht zwischen dem Handlungsort und der location. Während dies (bis jetzt) nur ein sekundärer Faktor für ein film tourism inducement ist hier ist vor allem der „Sehnsuchtsgrad“ der Zuschauer ausschlaggebend - verringert sich dadurch die Zufriedenheit der Filmtouristen vor Ort mitunter erheblich: Ein zu geringer Ikonizitätsgrad verhindert einen Wiedererkennungseffekt und macht damit auch eine „mental vision“, d. h. ein imaginäres Nacherleben einer Filmszene, unmöglich. Dass Filmtouristen derart überrascht und enttäuscht reagieren können, illustriert auch, dass sie beim Betrachten des Films aufgrund des Fotorealismus der digitalen Landschaften mehr reale Geografie, d. h. mehr location, im Handlungsort vermutet hatten, als in der Filmproduktion wirklich eingesetzt wurde.

Die Enttäuschung der Filmtouristen weist überdies auf ein weiteres zukünftiges filmtouristisches Problem hin: Ähnlich wie seit längerem aufgrund der digitalen Nachbearbeitung bzw. des „photoshoppings“ von Covermodeln auf Modemagazinen ein unrealistisches und unerreichbares Schönheitsideal propagiert wird, so könnte auch durch ständige digitale Landschaftsmanipulation ein unrealistisches „Landschaftsideal“ entstehen. ${ }^{97}$ Wenn unrealistische filmtouristische Erwartungen an die eingesetzten locations gestellt werden, könnte dies zukünftig dazu führen, dass das generelle filmtouristische Interesse aufgrund ständiger Enttäuschungen beträchtlich abnimmt und nur noch Filme Filmtourismus erzeugen können, bei denen der Zuschauer weiß, das sie ohne oder mit nur wenig CGI produziert wurden.

Auch ein Film der 7. Simulationsstufe kann in besonderen Fällen noch Filmtourismus auslösen, wenn zum einen die Begeisterung der Zuschauer für die dargestellte Welt sehr ausgeprägt ist und zum anderen Art und Umfang der eingesetzten Fototexturen eine Assoziierung mit einer realen Geografie noch zulassen, wie dies bei Avatar der Fall gewesen ist. Die filmtouristischen Folgen für Neuseeland sind aber bereits jetzt ersichtlich: Während Filme der 5. Simulationsstufe wie Lord of the Rings und The Hobbit vor allem aufgrund ihrer realen locations signifikanten Filmtourismus erzeugen konnten, haben weder King Kong noch Avatar Filmtourismus nach Neuseeland ausge-

\footnotetext{
${ }^{97}$ Auch die bei Hobbyfotografen in Mode gekommene HDR-Fotografie (HDR = High Dynamic Range) dient dazu, mittels digitaler Nachbearbeitung selbst wenig spektakuläre Orte auf Fotos dennoch malerisch und surreal wirken zu lassen, was beim Betrachter zu unrealistische Erwartungen an diese Orte führen kann.
} 
löst, obwohl diese Filme in Neuseeland produziert wurden und insbesondere bei Avatar auch Fototexturen aus der neuseeländischen Landschaft eingesetzt wurden. Dies könnte sich insofern für Neuseeland als problematisch erweisen, als dass einige Jahre nach Erscheinen von Lord of the Rings ein Nachlassen des filmtouristischen Interesses an Neuseeland spürbar wurde, dem nur durch den Dreh des Hobbits entgegengewirkt werden konnte. Bei den Fortsetzungen von Avatar ist kaum zu erwarten, dass zu einer mittlerweile bei Fantasyfilmen fast schon als „klassisch“ zu bezeichnenden Produktionsweise mit on location-Drehs in Neuseeland zurückgekehrt wird, wobei ausgerechnet Peter Jacksons WETA-Studios sich als ein treibender Motor hinter der Landschaftsdigitalisierung - und somit als direkter Konkurrent zu den neuseeländischen locations - erwiesen hat.

Im Vergleich zu den Filmen der 3. und 4. Simulationsstufe, wo eine reale Geografie durch eine andere reale Geografie ersetzt wird und viele Zuschauer ein erhebliches Problem mit der filmgeografischen Authentizität dieser Filme sehen, können Zuschauer bei Fantasyfilmen folglich die Ersetzung einer realen Geografie, die ohnehin eine fiktive Geografie simulieren soll, durch eine ebenso fiktive bzw. virtuelle digitale Geografie viel bereitwilliger akzeptieren. Eine geografische Realität durch eine andere geografische Realität zu ersetzen, stellt für Zuschauer folglich ein größeres Authentizitätsproblem dar als die Simulation einer fiktiven Geografie durch eine virtuelle Geografie. In filmtouristischer Hinsicht scheinen Filme der 3. und 4. Simulationsstufe aufgrund ihrer Doppelreferenzialität ein grundsätzlich geringeres Potenzial für Filmtourismus zu haben als Filme der 5. Simulationsstufe, da bei letzteren nur ein Referent in Form der realen location existiert und die Referenzialität somit „unidirektional“ ist.

Wie die Arbeit zeigen konnte, ist der Bezug zwischen filmischen und nationalen Räumen komplex, mehrschichtig und von der jeweiligen Handlungsort-location-Konstellation bzw. Simulationsstufe abhängig. Um auf eine in der Einleitung formulierte Feststellung zurückzukommen, konstruieren Filme Räume, die es ohne diese Filme nicht gäbe (Zimmerman 200ı: 229). Diese filmischen Räume werden zwar immer häufiger primär selbstreferenziell, sie beziehen sich aber noch häufig genug auf bestehende Diskurse um Raum, Nation und imaginierte nationale Räume, die sich während des nation building-Prozesses gebildet haben und/oder im Rahmen des nation branding produziert werden. Diese Diskurse werden durch die filmischen Raumkonstruktionen reproduziert, erweitert oder auch dekonstruiert. Dies geschieht, wie dargelegt, keinesfalls nur auf der Handlungsort-Ebene, sondern auch auf der location-Ebene, die als im Hintergrund wirkende ästhetische Instanz auch in Filmen Relevanz für die nationale Wertschöpfung hat, bei denen der Hand- 
lungsort sich von der location unterscheidet. Denn auch die location als materielle Basis einer Filmproduktion ist als Teil der Geografie eines Landes letztlich ebenso mit symbolischer Bedeutung aufgeladen, wie es Handlungsorte sind, und kann somit weiterverwertet werden.

Aus diesem Grund konnte die fiktive Geografie des filmischen „Mittelerdes“ trotz ihres fehlenden direkten Bezugs zur neuseeländischen Geografie aufgrund der eingesetzten neuseeländischen locations im Rahmen der $100 \%$ pure-Marketing-Kampagne verwertet werden. Wenn allerdings in Filmen mit fiktivem (und damit anationalem) Handlungsort die location durch vollständige Digitalisierung verschwindet, ist dieser filmisch konstruierte Raum letztlich von jeglichen Bezügen zu realen Nationen und materiellen Räumen entkoppelt: Dieser filmische Raum ist semiotisch „frei flottierend“ und rein selbstreferenziell geworden. Ein derartiger Raum kann nicht mehr genutzt werden, um im Rahmen der kulturellen und wirtschaftlichen Wertschöpfung den eigenen nationalen Raum mit neuen Konnotationen zu bereichern; solch ein filmischer Raum kann durch seine völlige Anationalität nicht mehr in Diskurse um nationale Räume einbezogen werden. Am oberen Ende des vom Simulationsstufenmodell nachgezeichneten Kontinuums befinden sich also Filme, die zwar noch Räumlichkeit simulieren, durch ihre fehlenden lebensweltlichen Bezüge aber eine digitale, „verflachte“ Räumlichkeit aufweisen, der die räumliche Dimension des Nationalen fehlt. Filme mit rein digitalen Räumen sind somit nicht nur entlokalisiert, sondern auch denationalisiert. Wenn aber ein als neuseeländisch identifizierbarer filmischer Raum - sei es der Handlungsort oder die location - einen wichtigen Faktor bei der Eingliederung eines Films in das neuseeländische Kino darstellt (wie in Kapitel 2 gezeigt), so entzieht sich ein fiktiver, digitaler und damit anationaler Raum dieser Zuordnungspraxis vollständig. Solche Filme können zwar noch über andere Faktoren der Produktionsebene (bspw. neuseeländische Filmemacher) in das neuseeländische Kinooeuvre inkorporiert werden - die räumliche Ebene dieser Filme ist der nationalen Wertschöpfung aber verlorengegangen. Mit der durch Globalisierungsprozesse vorangetriebenen Denationalisierung des filmischen Raums ist das Konstrukt des nationalen Kinos wieder ein Stück weit destabilisiert worden. 


\section{Ausblick und offene Forschungsfragen/Anwendungsbereiche}

Auf die Thematiken, die in dieser Arbeit behandelt worden sind, können keine abschließenden Antworten geliefert werden; es soll jedoch im Folgenden auf weitere Anwendungsbereiche der konzipierten Modelle und ein Ausblick auf mögliche zukünftige Entwicklungen gegeben werden. Die im Titel dieser Arbeit gestellte Frage nach einem Ende des nationalen Kinos kann im Falle Neuseelands, wie das erste Kapitel gezeigt hat, trotz durchaus zu beobachtender Destablisierungstendenzen grundsätzlich verneint werden. Es ist davon auszugehen, dass trotz der sich weiter intensivierenden Globalisierungsprozesse zumindest mittelfristig nationale Gefüge nicht einfach auseinanderbrechen und sich auflösen werden, da gerade in Krisenzeiten immer wieder protektionistische Maßnahmen ergriffen werden, um den eigenen Nationalstaat (vermeintlich) zu schützen. Aufgrund dieser Resilienz der Nationalstaaten werden höchstwahrscheinlich auch in Zukunft Filme - selbst diejenigen mit ausgesprochen transnationalem Produktionshintergrund - von Nationen im Rahmen politischer und kultureller Agenden immer wieder in die eigenen Konstruktionen nationaler Kinos eingebunden werden.

Während „große“ Kinos wie Hollywood und Bollywood für eine empirische Analyse, wie sie im zweiten Kapitel durchgeführt wurde, aufgrund ihrer Vielschichtigkeit und immensen Anzahl von Filmen kaum zu bewältigen wäre, könnte diese Analyseform gut auf kleinere nationale Kinos wie diejenigen Australiens, Islands oder Schwedens angewendet werden. Aufgrund des empirischen Ansatzes könnten die hierbei erhobenen Daten gut mit denen Neuseelands verglichen werden. Hierbei wäre hierbei interessant zu sehen, ob die Konsens-Dissens-Analyse einen wesentlich stärkeren oder schwächeren Konsens offenbart als im Falle des neuseeländischen Kinos, woraus sich Rückschlüsse über die „subjektive“ De- oder auch Renationalisierung der entsprechenden Kinos ziehen ließen. Eine im Anschluss folgende Konstellationsmatrix könnte in einem zweiten Schritt dann aufzeigen, ob auch in anderen Ländern die location einen derart wichtigen Faktor bei der Konstruktion eines nationalen Kinos darstellt und dadurch ebenfalls eine explizit filmgeografische Analyse der Filme angebracht erscheinen lässt. In einem letzten Schritt könnte dann die „objektive“ De- und Renationalisierung ermittelt und mit derjenigen im neuseeländischen Kino verglichen werden. Ist beispielsweise das isländische Kino resilienter gegenüber transnationalen Produktionspraktiken als das neuseeländische Kino oder ist es aufgrund seiner geografischen und ökonomischen Eingebundenheit in den europäischen Raum umso stärker auf ausländischen „Input" angewiesen? 
Der von Higson geforderten Berücksichtigung der Zuschauerschaft bei der Konstruktion eines nationalen Kinos ist in dieser Arbeit nur rudimentär in Form der Analyse des Anteils neuseeländischer Filme an den Top ıoo-Filmen am neuseeländischen Kinomarkt (siehe Abbildung 2.5.2) nachgekommen worden und könnte weiter ausgebaut werden. Durch zusätzliche Umfragen könnte der Frage nachgegangen werden, was in den Augen neuseeländischer Zuschauer einen „neuseeländischen Film“ ausmacht und was nicht. Allerdings ist hierbei zu berücksichtigen, dass, wie in der Einleitung erwähnt, nationales Kino in erster Linie top down konstruiert ist und nicht etwa demokratisch durch eine „Volksbefragung“ erfolgt. Dennoch könnte eine bottom up-Version des nationalen neuseeländischen Kinos auf interessante Weise mit den top down konstruierten Versionen kontrastiert werden.

Das im zweiten Kapitel entwickelte Simulationsstufenmodell ließe sich gut auf „landschaftsdominierte“ runaways, die die locations anderer Länder einsetzen, anwenden. Hierbei ließe sich der Frage nachgehen, ob beispielsweise australische locations einen höheren Aufwand bei der Delokalisierung erfordern als neuseeländische locations. Mit anderen Worten: Lässt sich ein Handlungsort wie Schottland einfacher mit einer neuseeländischen oder mit einer australischen location simulieren? Obwohl Hollywood-Filmemacher locations oftmals als beliebig austauschbar erachten, dürften doch bestimmte locations sich aufgrund ihrer geografischen Eigenschaften (Geomorphologie, Vegetation etc.) besser zur Simulation bestimmter Handlungsorte eignen als andere. ${ }^{98}$

Eine weitere Fragestellung wäre die, ob die im Zuge der Digitalen Revolution eingesetzte Virtualisierung und folglich Entlokalisierung von locations in gleichem Maße auch andere Länder betrifft, die sich als locations für runaway-Produktionen zur Verfügung stellen. Aufgrund der immensen praktischen und - wie diese Arbeit dargelegt hat - durchaus auch ästhetischen Vorzüge digital generierter Landschaften und Welten erscheint es mir sehr wahrscheinlich, dass auch andere Länder von dieser location-Virtualisierung betroffen sind oder bald sein werden und es sich folglich um ein globales Phänomen handelt. Dies gilt umso mehr, wenn man bedenkt, dass es durch die immer freier fließenden transnationalen Produktionsströme längst kein Problem mehr darstellt, beispielsweise einen in Schottland produzierten Film anschließend in weit entfernte „state of the

\footnotetext{
${ }^{98}$ So wäre es wohl prinzipiell möglich - insbesondere unter massiver Zuhilfenahme digitaler Effekte - mittels einer kongolesischen location den Handlungsort „Antarktis“ zu simulieren. Aufgrund der extremen geografischen Diskrepanz zwischen Kongo und Antarktis dürfte diese location/Handlungsort-Konstellation in der Praxis jedoch nicht vorkommen. Aus praktischen Gründen würde solch ein Film deshalb eher gleich vollständig in einem Filmstudio oder „kompatibleren“ Drehorten wie der Arktis gedreht werden.
} 
art“-Postproduction-Einrichtungen wie WETA digital oder Industrial Light and Magic auf digitalem Wege zu transferieren.

Des Weiteren bleibt abzuwarten, ob nicht in Zukunft sogenannte prozedurale Texturen, die tatsächlich ex nibilo im Computer generiert werden und somit überhaupt keine Spur von Indexikalität oder Ikonizität mehr aufweisen, die Fototexturen mit ihrem letzten Rest an Referenzialität ablösen werden und dann im Falle von Filmen der 7. Simulationsstufe tatsächlich von einer „desert of the geographic real“ gesprochen werden kann bzw. muss. In Anbetracht der Tatsache, dass im Zuge der Digitalen Revolution bereits die Trägermedien von Musik (mp3), Filmen (streaming) und mittlerweile auch Büchern (e-Books) komplett digitalisierbar geworden sind, erscheint es plausibel, auch von einer sukzessive zunehmenden Digitalisierung der einzelnen Produktionsschritte, die zur Produktion dieser Medien vonnöten sind, auszugehen.

Selbst auf die Digitalisierung und damit Virtualisierung des zentralsten „Elements“ einer Filmproduktion, nämlich der Schauspieler, scheint das Digitale überzugreifen: In einer ganzen Reihe von Arbeiten wird bereits über die Möglichkeit „synthetischer Darsteller“, die nicht mehr von menschlichen Schauspielern unterscheidbar sind, diskutiert (siehe z. B. Hoberg 1999: 33-34, North 2008: 2, Illiasch 2009: 85-88). Auch in den von mir behandelten Filmen, so z. B. bei Lord of the Rings und The Hobbit, haben bereits rein digitale Stuntdoubles agiert, ohne dass es den Zuschauern aufgefallen wäre. Wird also der Spielfilm durch das Digitale schließlich auch noch „entmenschlicht" werden? Meines Ermessens wird es viel eher zu einem flächendeckenden Einsatz rein digitaler Landschaften und Welten und damit Entlokalisierung kommen als zu einer derartigen „Entmenschlichung“ filmischer Produktionen - bevor der reale Mensch aus dem Film verschwindet, verschwindet erst der reale Raum. Denn aufgrund der sogenannten uncanny valley-Problematik ${ }^{99}$ dürfte die überzeugende digitale Nachbildung menschlicher Körpersprache und vor allem Mimik, ganz zu schweigen von der digitalen Nachbildung der menschlichen Stimme, noch bis auf weiteres Zukunftsmusik bleiben. Bis jetzt ist immer noch der Einsatz von motion capturing notwendig, um diese Aspekte des Menschseins glaubwürdig nachbilden zu können. Für motion capturing sind aber immer noch echte Schauspieler vonnöten, die mittels ihrer Bewegungen der digitalen Figur „authentische Lebendigkeit“ verleihen.

\footnotetext{
${ }^{99}$ Der von dem Roboteringenieur Masahiro Mori geprägte Begriff des uncanny valley beschreibt, wie ein immer menschenähnlicher gestalteter Roboter parallel dazu auch immer familiärer wirkt, sich diese Familiarität aber in Abneigung und Entfremdung umkehrt, wenn der Roboter zwar einem Menschen schließlich zum Verwechseln ähnlich sieht, aber doch weiterhin anhand subtiler, „unheimlicher“ Details von einem Menschen unterschieden werden kann. Wenn ein Roboter hingegen derart menschenähnlich ist, dass er überhaupt nicht mehr von einem Menschen unterschieden werden kann, also gewissermaßen die perfekte Simulation eines Menschen darstellt, ist die uncanny valley überwunden, da das Fremde dann als das Eigene akzeptiert wird (Mori, 1970).
} 
Im Vergleich zu der Frage nach dem Ende des neuseeländischen Kinos muss die Frage nach dem Ende der neuseeländischen location - und damit einem wichtigen Teil des nationalen Kinos - zumindest im Hinblick auf effektlastige Fantasyfilme folglich deutlich „pessimistischer“ ausfallen. Filme wie King Kong und Avatar beweisen, dass keine locations mehr für die Produktion solcher Filme benötigt werden und der Einsatz neuseeländischer locations im Hobbit vor allem ein $\mathrm{Zu}$ geständnis an die neuseeländische Tourismusindustrie darstellte, nicht aber eine unabdingbare Notwendigkeit gewesen ist. Insofern erscheint jetzt, im Jahre 20I5, das 201 von Leotta - auch im Hinblick auf die damals noch ausstehende Ausstrahlung der Hobbit-Filme - gezeichnete Bild vom zukünftig zu erwartenden Filmtourismus in Neuseeland zu optimistisch, wenn er schreibt: „[...] while local films will continue to shape New Zealand's virtual geography, global audiences will still undertake both imaginary and physical filmic tours of the country“ (20II: 204). Denn mittlerweile werden zunehmend sogar in Genres wie Kriminalfilmen (z. B. David Finchers Zodiac [2007] und Clint Eastwoods Changeling [2008]) digitale Landschaften eingesetzt, was darauf hindeutet, dass die CGI in Zukunft auch geografisch „realistischere“ Filmgenres erobern wird und somit nicht nur die 5. durch die 7. Simulationsstufe, sondern zunehmend auch Filme der 3. und 4. Simulationsstufe durch die 6. Simulationsstufe ersetzt werden könnten. Es ist abzusehen, dass zumindest mittelfristig statt auf Filme mit neuseeländischer location aber ausländischem, realitätsbasiertem Handlungsort auch hier vermehrt gleich auf digitale Landschaften zurückgegriffen werden wird, sodass auch hier die neuseeländische location obsolet werden könnte.

Ist somit zu erwarten, dass der Filmtourismus nach Neuseeland stark einbrechen wird? Wenn man die Digitalisierung verschiedener Bereiche betrachtet, fällt auf, dass zwar beispielsweise $\mathrm{mp} 3$ sich zu einem Standard in puncto Musik entwickelt hat, dass aber weiterhin immer noch $\mathrm{Mu}$ sik-CDs verkauft werden. Sogar ein Retro-Trend ist hierbei zu beobachten, wo neuerdings nicht nur das Sammeln, sondern auch das Abspielen (mit den dafür notwendigen Geräten) alter VinylSchallplatten wieder „in“ geworden ist. Bemerkenswert ist hier, dass Schallplatten somit nicht einfach als „Relikte der Vergangenheit“ in Schaukästen gestellt werden, sondern auch in ihrer ursprünglichen Funktionalität „reaktiviert“ werden - sie sind somit nicht einfach (disfunktionales) „Heritage“ im Sinne Kirshenblatt-Gimbletts (1995: 370), sondern wieder zu Gebrauchsgegenständen geworden. Vielleicht liegt in dieser in vielen Lebensbereichen zu beobachtenden nostalgischen Rückbesinnung auf „Altes“ auch eine Hoffnung für die neuseeländische location. So wie neuerdings Heimatfilme (z. B. die Sebnsuchtsland-Reihe im ZDF) und Heritagefilme wieder an Beliebt- 
heit gewonnen haben, könnte vielleicht auch Neuseeland aufgrund seines clean É green-Images sich als location für Filme vermarkten, die „alte Werte“ vermitteln und der Sehnsucht vieler Menschen nach pastoraler Idylle in einer „heilen Welt“ Ausdruck verleihen. Wenn langfristig landschaftliche Digitalität der Standard geworden ist, könnte sich Neuseeland womöglich eine Marktnische als location für Filme, die nicht nur inhaltlich nostalgische Themen behandeln, sondern auch im Rahmen ihrer Vermarktung mit dem Einsatz „traditioneller“ Produktionsmethoden unter Einsatz „echter“ locations werben und damit an die Sehnsucht des Menschen nach dem Realem, dem Echten, dem Authentischen, in Zeiten der allgegenwärtigen Simulation und Digitalität appellieren. 


\section{Literaturverzeichnis}

\section{Sekundärliteratur}

Anderson, Benedict R. Imagined Communities: Reflections on the Origin and Spread of Nationalism. 2. Auflage. London: Verso, 1991. Print.

Barthes, Roland. „Rhetoric of the Image.“ 1964. Image Music Text: Roland Barthes. Übers. und Hrsg. Stephen Heath. London: Fontana Press, 1977. 32-51. Print.

Baudrillard, Jean. „Simulacra and Simulations.“ 1981. Übers. Paul Voss; Paul Patton; Philip Beitchman. Jean Baudrillard: Selected Writings. Hrsg. Mark Poster. Stanford: Stanford UP, 1988. 166-184. Print.

Beeton, Sue. Film-Induced Tourism. Clevedon: Channel View Publications, 2005. Print.

Bell, Claudia. „Branding New Zealand: the National Green-wash.“ British Review of New Zealand Studies 15.6 (2005). 13-28. Print.

Bell, Claudia; Steve Matthewman, Hrsg. Cultural Studies in Aotearoa New Zealand: Identity, Space and Place. Victoria: Oxford, 2004. Print.

Bendix, Regina. In Search of Authenticity: The Formation of Folklore Studies. Madison: Wisconsin UP, 1997. Print.

Billig, Michael. Banal Nationalism. 2. Auflage. London: SAGE, 1997. Print.

Boorstin, Daniel J. The Image: A Guide to Pseudo-Events in America. 196r. New York: Vintage Books, 2012. Print.

Box Office Mojo. Web. is. November 2012. <www.boxofficemojo.com $>$.

Brodie, Ian. Lord of the Rings Location Guide Book. 2. Auflage. Vorwort von Peter Jackson. Auckland: Harper Collins, 2003. Print.

Carl, Daniela Susann. Cultural Representation of New Zealand's Landscapes in the Films of The Lord of the Rings and its Implications for Tourism. Masterarbeit Univ. Wellington, 2004. Web. 16. Oktober 2013. <http://hdl.handle.net/10o63/394>.

Cameron, Allan. "The Locals and the Global: Transnational Currents in Contemporary New Zealand Horror." Studies in Australasian Cinema 4.I (2010). 55-72. Web. 30. April 2014. $<$ http://dx.doi.org/10.1386/sac.4.I.55 I $>$.

Cobb, Russell, Hrsg. The Paradox of Authenticity in a Globalized World. New York: Palgrave Macmillan, 20I4. Print. 
Conrich, Ian. „New Zealand Gothic.“ A New Companion to the Gothic. Hrsg. David Punter. 393408. Print.

Conrich, Ian; Stuart Murray, Hrsg. Contemporary New Zealand Cinema: From New Wave to Blockbuster. London: I.B. Tauris Pub., 20o8. Print.

Conrich, Ian; Stuart Murray. Introduction. Contemporary New Zealand Cinema: From New Wave to Blockbuster. London: I.B. Tauris Pub., 2008. Print. I-I4.

Crofts, Stephen. „Concepts of National Cinema.“ The Oxford Guide to Film Studies. Hrsg.John Hill; Pamela Church Gibson. Oxford: Oxford UP, 1998. 385-394. Print.

Daur, Ute. Authentizität und Wiederholung: künstlerische und kulturelle Manifestationen eines Paradoxes. Bielefeld: transcript-Verlag, 20I3. Print.

Davis, Geoffrey V., Hrsg. Towards a Transcultural Future: Literature and Society in a 'Post'-Colonial World. Band I. Amsterdam: Rodopi, 2004. Print.

Desai, Jigna. „Bombay Boys and Girls. The Gender and Sexual Politics of Transnationality in the New Indian Cinema in English." Transnational Cinema: The Film Reader. Hrsg. Elizabeth Ezra; Terry Rowden. Abingdon: Routledge, 2006. 57-70. Print.

Drechsel, Paul; Bernhard Gölz; Bettina Schmidt. Kultur im Zeitalter der Globalisierung: Von Identität zu Differenzen. Frankfurt am Main: IKO-Verlag, 20oo. Print.

Duff, Alan. „Jacksons Traumwelt, eine Denunziation der Wirklichkeit.“ Süddeutsche Zeitung. 24. Dezember 20I2. Web. 4. August 20I5. <www.sueddeutsche.de/kultur/hobbit-film-inneuseeland-jacksons-traumwelt-eine-denunziation-der-wirklichkeit-I.I558209>.

Duncan, Jody; Lisa Fitzpatrick. The Making of Avatar. New York: Abrams, 2oro. Print.

Durovicova, Natasa; Kathleen Newman, Hrsg. World Cinemas, Transnational Perspectives. New York: Routledge, 2oIo. Print.

Eco, Umberto. Im Wald der Fiktionen: Sechs Streifzüge durch die Literatur. 1994. Übers. Burkhart Kroeber. Wien: Carl Hanser, 1994. Print.

„Errors in geography.“ IMDB. o. J. Web. 28. November 20I4. < www.imdb.com/title/ tto36475I/goofs?item $=$ gfo88I231 $>$.

Escher, Anton; Stefan Zimmermann. „Geography meets Hollywood. Die Rolle der Landschaft im Spielfilm." Geographische Zeitschrift 89.4 (200I): 227-236. Web. Io. April 20I4. < www.staff.uni-mainz.de/escher/pub/Neuer\%2oOrdner/ Geography\%2omeets\%2oHollywood.pdf>. 
Ezra, Elizabeth; Terry Rowden, Hrsg. Transnational Cinema: The Film Reader. Abingdon: Routledge, 2006. Print.

„Feature Films since 1939 by year to Nov 2012.“ Web. 21. November 2012. $<$ www.nzfilm.co.nz/sites/nzfc/files/Feature\%2oFilms\%2osince\%201939\%2 oby\%2oyear\%2oas\%20at\%2O2I\%2oNov\%2O2OI2.pdf>.

Genette, Gérard. Das Buch vom Beiwerk des Buches. 1987. Übers. Dieter Honig. Frankfurt a. M.: Campus, 1989. Print.

George, Sandy. „Jackson Report Critical of New Zealand Film Commission.“

Screendaily.com. I. Juli 20I0. Web. 27. November 2012.<www.screendaily.com/news/ asia-pacific-news/jackson-report-critical-of-new-zealand-film-commission/sors635.article > .

Goldberg, Matt. „King Kong Returns to Universal Studios Hollywood for New 3 D Ride Created by Peter Jackson.“ I. Februar 20I0. Web. 19. Juni 2015. <http://collider.com/kingkong-returns-to-universal-studios-hollywood-for-new-3d-ride-created-by-peter-jackson/>. Gunning, Tom. „The Cinema of Attraction: Early Film, Its Spectator and the Avant-Garde.“ Wide Angle 8.3-4 (1986): 63-70. Web. I2. Januar 2014.<www.columbia.edu/itc/ film/gaines/historiography/Gunning.pdf >.

Handel, Jonathan. The New Zealand Hobbit Crisis: How Warner Bros. Bent a Government to Its Will and Crushed an Attempt to Unionize The Hobbit. Los Angeles: Hollywood Analytics, 20I2. Print.

Hattendorf, Manfred. Dokumentarfilm und Authentizität: Ästhetik und Pragmatik einer Gattung. 2. Auflage. Konstanz: UVK Medien, 1999. Print.

Hardy, Ann. „Beyond Materialism? Spirituality and Neo-Utopian Sensibility in Recent New Zealand Film" Contemporary New Zealand Cinema: From New Wave to Blockbuster. Hrsg. Ian Conrich und Stuart Murray. London: I.B. Tauris Pub., 2008. Print. II9-I34. Hayward, Susan. French National Cinema. London: Routledge, 1993. Print. Heath, Stephen, Hrsg. und übers. Image Music Text: Roland Barthes. London: Fontana Press, 1977. Print.

Herzfeld, Michael. Cultural intimacy : social poetics in the nation-state. New York/London: Routledge, 2005. Print.

Higson, Andrew. „The Concept of a National Cinema.“"1989. Film and Nationalism. Hrsg. Alan Williams. New Brunswick: Rutgers UP, 2002. 52-67. Print. 
Higson, Andrew. Waving the Flag: Constructing a National Cinema in Britain. Oxford:

Clarendon Press, 1995. Print.

Hill, John. „The Issue of National Cinema and British Film Production.“ New Questions of British Cinema. Hrsg. Duncan Petrie. London: BFI Publishing, 1992. IO-2I. Print.

Hill, John; Pamela Church Gibson, Hrsg. The Oxford Guide to Film Studies. Oxford: Oxford UP, 1998. 385-394. Print.

Hjort, Mette; Scott MacKenzie, Hrsg. Cinema and Nation. 2. Auflage. London: Routledge, 2002. Print.

Hoberg, Almuth. Film und Computer: Wie digitale Bilder den Spielfilm verändern.

Diss. Univ. Hamburg, 1988. Frankfurt a. M.: Campus, 1999. Print.

Hokowhitu, Brendan. „Understanding Whangara: Whale Rider as Simulacrum.“ New

Zealand Journal of Media Studies 10:2 (2007). Web. 2. Februar 2013.

<www.nzmediastudies.org.nz/articles/osIoo2 hokowhitu.pdf $>$.

Houser, Nathan, Hrsg. Writings of Charles S. Peirce: A Chronological Edition. Band 2

(I867-1871). Bloomington: Indiana UP, 1984. Print.

Huffstutter, P. J. „Not Just a Tolkien Amount.“ L. A. Times. 24. Oktober 2003. Web. I. August 2015. <http://articles.latimes.com/2003/oct/24/business/fi-frodoecon24>.

Huijibens, Edward H. „Nation-Branding: A Critical Evaluation. Assessing the Image Building of Iceland." Iceland and Images of the North. Hrsg. Sumarliði Ísleifson. Québec: Presses de l’Université du Québec, 20II. 553-582. Print.

IMDb. Web. I5. November 2012. < www.imdb.com>.

Illiasch, Therese. Aus alt mach neu - King Kong einst und jetzt: Was sich in 70 Jabren

Filmgeschichte getan hat, anhand der Filme King Kong und die weiße Frau (I933) und King Kong. Ein Blick in die Technik von Hollywood. Diplomarbeit Univ. Wien, 2009. Web.

I5. Oktober 2014. < http://othes.univie.ac.at/7070/I/2009-IO-20 0420122.pdf >.

Ísleifson, Sumarliði, Hrsg. Iceland and Images of the North. Québec: Presses de l'Universite du Québec, 20Ir. Print.

Jackson, Peter. „Foreword“. Vorwort zu Lord of the Rings Location Guide Book. Ian Brodie. Auckland: Harper Collins, 2003. 6-7. Print.

Jutel, Thierry. „Lord of the Rings: Landscape, Transformation, and the Geography of the Virtual.“ Cultural Studies in Aotearoa New Zealand: Identity, Space and Place. Hrsg. Claudia Bell; 
Steve Matthewman. Victoria: Oxford, 2004. 54-65. Print.

Kaschuba, Wolfgang; Thomas Scholze; Leonore Scholze-Irrlitz, Hrsg. Alltagskultur im Umbruch.

Weimar: Böhlau, 1996. Print.

Kirshenblatt-Gimblett, Barbara. Ethnomusicology.39.3 (Herbst 1995):367-380. Print.

Laure-Ryan, Marie. Possible Worlds, Artificial Intelligence, and Narrative Theory. Bloomington: Indiana UP, 199I. Print.

Leotta, Alfio. Touring the Screen. Bristol: Intellect, 2oII. Print.

Linder, Rolf. „Arbeiterkultur und Authentizität.“ Alltagskultur im Umbruch. Hrsg. Wolfgang Kaschuba; Thomas Scholze; Leonore Scholze-Irrlitz. Weimar: Böhlau, 1996: 7I-80.

Lukinbeal, Chris. „The map precedes the territory: An introduction to essays in cinematic geography." GeoJournal 59 (2004): 247-25I. Print.

Lukinbeal, Chris; Stefan Zimmermann. „Film Geography: A New Subfield.“ Erdkunde 6o (2006). 315-325. Web. 2. Januar 2015. <www.erdkunde.uni-bonn.de/ archive/2006/film-geographya-new-subfield/at download/attachment>.

MacCannell, Dean. The Tourist: A New Theory of the Leisure Class. Neuauflage. Los Angeles: California UP, 1999. Print.

Manovich, Lev. The Language of New Media. Cambridge: MIT Press, 20oI. Print.

Martin, Helen; Sam Edwards. New Zealand Film: 1912 - 1996. Auckland: Oxford UP, 1997. Print.

Martin, Josh. „Lobbyist Slams Scientists Over NZ's Environmental Record.“ New Zealand Herald. 22. November 20I2. Web. II. November 20I4. <www.nzherald.co.nz/

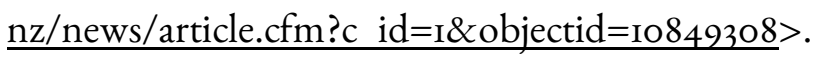

Masters, Catherine. „Battling Actors Have a Hobbit Onside.“ New Zealand Herald. 2. Oktober 20I0. Web. 17. Juni 20I5. <www.nzherald.co.nz/nz/news/ article.cfm?c id=I\&objectid $=10677637>$.

Mayer, Geoff; Keith Beattie, Hrsg. The Cinema of Australia and New Zealand. London: Wallflower Press, 2007. Print.

Mette, Jean-Luc. „Depressionswelle nach 'Avatar'“ $W A Z$. 29. Januar 20Io. Web. 20. Juni 2015. $<$ www.derwesten.de/kultur/depressionswelle-nach-avatar-id3310586.html $>$.

Metz, Christian. Semiologie des Films. München: Fink, 1972. Print.

Metz, Christian. Sprache und Film. Frankfurt a. M.: Athenäum Verlag, 1973. Print. „Milford.“ Google Maps Streetview-Darstellung. Web. I4. Juni 20I4. < www.google.de/maps/ 
@-44.6720196,167.9287747,3a,42.7y,294.83h,105.06t/data=!3m6!IeI!3m4!IsTUAawsJcYb7x XsanaXJvwA!2eo! 7 i13312!8i6656>.

Miller, Gerri. „Inside ,The Water Horse’.“ HowStuffWorks. 2I. Dezember 2007. Web.

2I. August 20I4. <http://entertainment.howstuffworks.com/water-horsez.htm $>$.

Mittell, Jason. „A Cultural Approach to Television Genre Theory.“ Cinema Journal 40.3 (200I): 3-23. Print.

Mori, Masahiro. The Uncanny Valley. 1970. Übers. Karl F. MacDorman and Takashi Minato. 2005. Web. I5. August 2015. <www.androidscience.com/theuncannyvalley/ proceedingszoos/uncannyvalley.html >.

Murray, Stuart. „Precarious Adulthood': Communal Anxieties in 1980s Film. “ Contemporary New Zealand Cinema: From New Wave to Blockbuster. Hrsg. Ian Conrich und Stuart Murray. London: I.B. Tauris Pub., 2008. Print. 169-I80.

„New Zealand Film Commission Act 1978.“ Web. I. November 2012. <www.legislation.govt.nz/act/public/1978/oo6I/latest/DLM22640.html>.

„New Zealand, Home of Middle-earth.“ Web. I4. März 20I5. $<$ www.youtube.com/watch?v=RRA eVAyH 5 c $>$. Videoclip (6:37 min.).

„New Zealand in Profile: 2013“. Web. I2. Juni 2013.

$<$ www.stats.govt.nz/browse for stats/snapshots-of-nz/nz-in-profile-2013/ about-new-zealand.aspx $>$.

New Zealand Screen Council. Overview of the New Zealand Screen Production Sector, November 2005. Web. I2. Juni 2013. $<$ www.sfu.ca/cmns/courses/230/Body\%2otext.section/New\%2oZealand/ NZSC production\%2osector\%2ooverview.pdf $>$.

North, Dan. Performing Illusions: Cinema, Special Effects and the Virtual Actor. London: Wallflower Press, 2008. Print.

Nye, David E. American Technological Sublime. Cambridge: MIT Press, 1994. Print. „NZ lake in Nessie film boosts Scottish tourism.“ The Dominion Post. 2. November 2008.

Web. I4. August 20I4. < www.stuff.co.nz/entertainment/263550/

NZ-lake-in-Nessie-film-boosts-Scottish-tourism $>$. 
Ogden, Charles; Ivor Armstrong Richards. The Meaning of Meaning: A Study of the Influence of Language Upon Thought and the Science of Symbolism. 1923. London: Routledge, 20oI. Print.

Peirce, Charles Sanders. „On a New List of Categories.“ 1867 . Writings of Charles S. Peirce: A Chronological Edition. Band 2 (1867-1871). Hrsg. Nathan Houser. Bloomington: Indiana UP, 1984. 49-59. Print.

Petrie, Duncan, Hrsg. New Questions of British Cinema. London: BFI Publishing, 1992. Print.

Petrie, Duncan. Shot in New Zealand: The Art and Craft of the Kiwi Cinematographer.

Auckland: Random House, 2007. Print.

Phillips, Jock. „Perceptions of the landscape.“ Te Ara - the Encyclopedia of New Zealand.

2. November 20I2. Web. I. August 20I5. <www.TeAra.govt.nz/en/ perceptions-of-the-landscape $>$.

Piatti, Barbara. Die Geographie der Literatur : Schauplätze, Handlungsräume, Raumphantasien. Göttingen : Wallstein, 2008. Print.

Piazza, Jo. „Audiences experience 'Avatar' blues.“ CNN.com. II. Januar 20Io. Web. 20. Juni 2015. < http://edition.cnn.com/2oro/SHOWBIZ/Movies/oI/II/ avatar.movie.blues/index.html>.

Piturro, Vincent. The Audience and the Film: A Reader-response Analysis of Italian

Neorealism. Diss. Univ. Colorado, 2008. Web. 2. Februar 2015.

$<$ http://dissexpress.umi.com/dxweb/doc/

304620908.html?FMT $=$ AI\&desc $=$ The + audience + and + the + film\%3 $\mathrm{A}+\mathrm{A}+$ reader response+analysis+of+Italian+Neorealism $>$. Microfiche: UMI 330388I.

Poster, Mark, Hrsg. Jean Baudrillard: Selected Writings. Stanford: Stanford UP, 1988. Print.

Power, Ed. „'This Is Not Middle-Earth': New Zealand is exactly the wrong backdrop for the works of J.R.R.Tolkien.” Slate.com. I2. Dezember 2013. Web. I5. Juni 2015. $<$ www.slate.com/articles/arts/culturebox/2013/12/the hobbit and lord of the rings movies new zealand was the wrong filming.html>.

Prince, Stephen. Digital Visual Effects in Cinema: The Seduction of Reality. New Brunswick: Rutgers UP, 20I2. Print.

„'Prove another park inspired Avatar and we'll give you £Io,ooo': Chinese national forest issues challenge as rival province claims it was true source of film." Daily Mail. 
03. August 2012. Web. 20. Juni 2015. <www.dailymail.co.uk/news/ article-2182690/Chinese-national-park-offers-IO-ooo-challenge-disprove-claiminspiration-Avatar.html\#ixzzzeooouGYc $>$.

Punter, David, Hrsg. A New Companion to the Gothic. West Sussex: Blackwell Publishing, 20I2. Print.

Read, Lynette. Vincent Ward: The Emergence of an Aesthetic. Diss. U of Auckland, 2004. Web.

I. Feb 20I4. < https://researchspace.auckland.ac.nz/bitstream/handle/2292/1509/ o2whole.pdf?sequence $=$ IO $>$.

„Raetihi.“ Google Maps Streetview-Darstellung. Web. II. Juni 2014. $<$ www.google.de/maps/@-39.426985,175.279463,3a,75y,240.25h,78.88t/

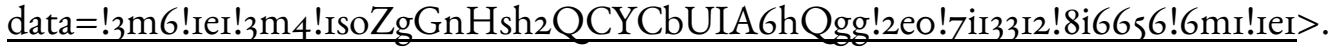

Richter, Sebastian. Digitaler Realismus: Zwischen Computeranimation und Live-Action. Die neue Bildästhetik in Spielfilmen. Bielefeld: transcript, 2008. Print.

Robertson, Barbara. „CG In Another World.“ Computer Graphics World 32.12 (Dez. 2009). Web. I2. Dezember 20I4. <www.cgw.com/Publications/CGW/2009/ Volume-32-Issue-I2-Dec-2009-/CG-In-Another-World.aspx $>$.

Roddick, Nick. „Boom and Bust: Tax-driven Film Production in New Zealand in the 1980s.“ Hrsg. Ian Conrich; Stuart Murray, Contemporary New Zealand Cinema: From New Wave to Blockbuster. London: I.B. Tauris Publishing, 2008. 37-48. Print.

Roesch, Stefan. The Experience of Film Location Tourists. Bristol: Channel View, 2009. Print.

Rössner, Michael; Uhl, Heidemarie, Hrsg. Renaissance der Authentizität? Über die neue Sebnsucht nach dem Ursprünglichen. Bielefeld: transcript-Verlag, 2012. Print

Rotten Tomatoes. Web. I5. November 2012. < www.Rottentomatoes.com $>$.

„Set jetting around New Zealand locations.“ New Zealand Tourism. o. J. Web. 22. April 20I5. $<$ http://media.newzealand.com/en/story-ideas/set-jetting-around-new-zealandlocations/>.

Shin, Mina. „Making a Samurai Western: Japan and the White Samurai Fantasy in The Last Samurai."Journal of Popular Culture 43.5 (2010). Web. I4. Juli 2014.

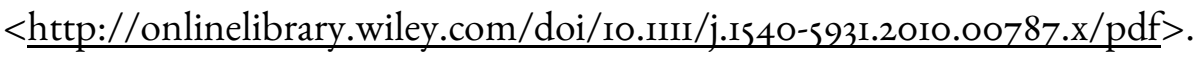

Simmons, Rochelle. „The Piano.“ The Cinema of Australia and New Zealand. Hrsg. 
Geoff Mayer; Keith Beattie. London: Wallflower Press, 2007. I49-155. Print.

Thomas, Liz. „The Avatar Effect: Movie-goers Feel Depressed and Even Suicidal at not Being Able to Visit Utopian Alien Planet.“ Daily Mail. I2. Januar 2oro. Web. 2o. Juni 2015. $<$ www.dailymail.co.uk/news/article-I242409/

The-Avatar-effect-Movie-goers-feel-depressed-suicidal-able-visit-utopian-alienplanet.html>.

Tooke, Nichola; Michael Baker. „Seeing Is Believing: The Effect of Film on Visitor Numbers to Screened Locations." Tourism Management 17.2 (1996). 87-94. Web.

I3. April 2015. <www.sciencedirect.com.33II.han.sub.uni-goettingen.de/science/ journal/02615177/17/2>.

Urry, John. The Tourist Gaze: Leisure and Travel in Contemporary Societies. London: SAGE, 1990. Print.

Vieira, Joao Luiz. „The Transnational Other: Street Kids in Contemporary Brazilian Cinema". World Cinemas, Transnational Perspectives. Hrsg. Natasa Durovicova; Kathleen Newman. New York: Routledge, 20I0. 226-244. Print.

„Wakatipu Lake.“ Encyclopaedie Britannica. Web. 3. März 20I5.

$<$ www.britannica.com/EBchecked/topic/63425I/Wakatipu-Lake $>$.

Wake, Jenny. The Making of King Kong: The Official Guide to the Motion Picture. New York: Pocket Books, 2005. Print.

Wallis, Mike. Directors Statement. o. J. Web. 3. März 20I5. $<$ www.goodfornothingmovie.com/directors-statement/ $>$.

Williams, Alan, Hrsg. Film and Nationalism. New Brunswick: Rutgers UP, 2002. Print.

Wilson, Janet. „New Zealand Literary Nationalism and the Transcultural Future. Or: Will the Centre Hold?" Hrsg. Geoffrey V. Davis. Towards a Transcultural Future: Literature and Society in a 'Post'-Colonial World. Band I. Amsterdam: Rodopi, 2004. II9-I34. Print.

Zimmermann, Stefan; Anton Escher. „Geography meets Hollywood: Die Rolle der Landschaft im Spielfilm." Geographische Zeitschrift 89.4 (2001): 227-236. Web. Io. April 20I4. < www.staff.unimainz.de/escher/pub/Neuer\%2oOrdner/ Geography\%2omeets\%2 oHollywood.pdf>. 
Zimmermann, Stefan. Wüsten, Palmen und Basare: Die cineastische Geographie des imaginierten Orients. Diss. Univ. Mainz, 2007. Web. 17. Oktober 2014. $<$ http://ubm.opus.hbz-nrw.de/volltexte/20II/2728/pdf/doc.pdf $>$.

\section{Filme}

Avatar. Reg. James Cameron. 2oth Century Fox Home Entertainment, 2009. Film. Good for Nothing. Reg. Mike Wallis. Screen Media Films, 2oIr. Film.

-.-. DVD-Cover. Good for Nothing.

---. Making Of. Good for Nothing.

Lord of the Rings: Fellowship of the Ring. Reg. Peter Jackson. Warner Video Home, 2001.

Film.

Lord of the Rings: Two Towers. Reg. Peter Jackson. Warner Video Home, 2002. Film.

Lord of the Rings: Return of the Ring. Reg. Peter Jackson. Warner Video Home, 2003. Film.

King Kong. Reg. Peter Jackson. Universal Studios Home Entertainment, 2005. Film.

Last Samurai. Reg. Edward Zwick. Warner Home Video, 2003. Film.

---. Audiokommentar. Last Samurai.

--. Making Of. Last Samurai.

The Waterhorse: Legend of the Deep. Reg. Jay Russell. Sony Pictures Home Entertainment, 2007.

Film.

---. „Featurettes: Setting the Scene.“ Making Of. Legend of the Waterhorse.

The Hobbit: An Unexpected Journey. Reg. Peter Jackson. Warner Home Video, 20II. Film.

The Hobbit: Desolation of Smang. Reg. Peter Jackson. Warner Home Video, 2012. Film.

The Hobbit: The Battle of the Five Armies. Reg. Peter Jackson. Warner Home Video, 20I3. Film.

Without a Paddle. Reg. Steven Brill. Paramount Home Entertainment, 2004. Film.

---. Audiokommentar. Without a Paddle.

Vertical Limit. Reg. Martin Campbell. Columbia Tristar, 20oo. Film.

---. Audiokommentar. Vertical Limit.

---. „Surviving the Limit“. Making Of. Vertical Limit

Willow. Reg. Ron Howard. 2oth Century Fox Home Entertainment, 1988. Film. 


\section{Interviews}

Brill, Steven. „Steven Brill - Without a Paddle Interview.“ Interview. Tribute. o. J. Web.

I4. April 20I5. < www.tribute.ca/interviews/steven-brill-without-a-paddle/ director/7774/>. Videoclip (3:40 min.).

Brill, Steven. The Steven Brill Interview by Mike Smith. Interview von Mike Smith. 2004. Web. I4. April 2015.<www.crazedfanboy.com/spotlight/stevenbrill.html>.

Brill, Steven. Without a Paddle. Interview von Gareth von Kallenbach. II. August 2004.

Web. I4. April 20I5. <www.leesmovieinfo.net/article.php?a=\$27>.

Cameron, James. „Star-Regisseur Cameron: ,Wir entwickeln uns zur Avatar-Gesellschaft‘.“ Interview von Lars-Olav Beier. Spiegel Online. 21. Dezember 2009. Web. 14. Juni 2015. $<$ www.spiegel.de/kultur/kino/star-regisseur-cameron-wir-entwickeln-uns-zuravatar-gesellschaft-a-667576.html > .

Muren, Dennis. „Dennis Muren interview: How Willow changed the movies.“ Interview von Daisy Bowie-Sell. The Telegraph. I8. März 2013. Web. 28. Juni 2015. <www.telegraph.co.uk/culture/film/starsandstories/9937074/ Dennis-Muren-interview-How-Willow-changed-the-movies.html>.

Narbey, Leon. „ScreenTalk Interview with Leon Narbey.“ Interview von Andrew Whiteside. Nzonscreen.com. 20II. Web. 23. April 2014. <http://screentalk.nzonscreen.com/interviews/ leon-narbey-illustrious-cinematographer; https://www.youtube.com/watch?v=ZezeXNHehUg>. Videoclip (9:53 min.).

Wallis, Mike. „Good for Nothing: An Interview with Mike Wallis.“ Interview von Matt Kettmann. Santa Barbara Independent. 28. Januar 20oI. Web. I4. April 2015. <www.independent.com/news/20II/jan/28/emgood-nothingem/>.

Zwick, Ed.“An Interview With The Director And Cast Of The Last Samurai.“ Interview von Jeff Otto.“ IGN3. Dezember 2003. Web. I4. April 20I5. <www.ign.com/articles/ 2003/12/03/an-interview-with-the-director-and-cast-of-the-last-samurai $>$.

\section{Rezensionen und Foreneinträge}

„(poorlittlerichboy@hotmail.com)“. „A superb epic.“ Rez. von Last Samurai, Reg. Edward Zwick. I5. Januar 2004. Web. 19. Juni 20I5. <www.imdb.com/title/tto325710/ reviews? count $=917 \&$ start $=19>$. 
„235SCOPE“. „Not a Top Campbell but a Worthy Second-Placer.“ Rez. von Vertical Limit, Reg. Martin Campbell. Io. Dezember 2000. Web. I4. April 2015. <www.imdb.com/title/ ttolgo $865 /$ reviews? count $=373 \&$ start $=139>$.

„amleeparker“. „Not filmed in Scotland.“ Foreneintrag zu The Waterhorse: Legend of the Deep,

Reg. Jay Russell. 2I. Januar 2008 09:15:37. Web. 19. Juni 2015. <www.imdb.com/title/ tto760329/board/nest/94499719?d=95429147\#95429147>.

„Amy Adler“. „Superb family film, with a great story and a wonderful production.“ Rez. von

The Waterhorse: Legend of the Deep, Reg. Jay Russell. I6. Februar 2008. Web.

I4. April 2015. <www.imdb.com/title/tto760329/reviews?count=84\&start=62 > .

„avrilyn“. „Here is a story that will break your heart.“ Rez. von Lord of the Rings: Die

Gefährten, Reg. Peter Jackson. 22. Dezember 200I. Web. 4. Februar 2015.

<www.amazon.com/review/RGM7oAWKFAWI4>.

„Benjamin Cox“. „Finally, an Eighties fantasy film that isn't rubbish - 73\%.“, Rezension von

Willow, Reg. Ron Howard. 20. September 2005. Web. I3. Juni 2015. <www.imdb.com/

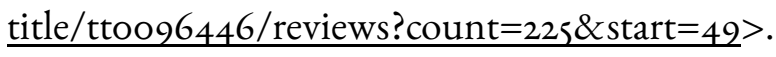

„blackmage439“. „Absolutely Amazing and Immersive.“ Rezension von Avatar, Reg. James Cameron. II. Februar 20I0. Web. 20. Juni 20I5. <www.imdb.com/title/tto499549/ reviews? $\operatorname{start}=1540>$.

„contact-282“. „Not filmed in Scotland.“ Foreneintrag zu The Waterhorse: Legend of the Deep,

Reg. Jay Russell. I5. April 2008 21:II:40. Web. 19. Juni 2015. <www.imdb.com/title/ tto760329/board/thread/94499719?d=103379263\#103379263>.

„cseon“. „Awful New Zealand Western Homage.“ Rez. von Good for Notbing. Reg. Mike

Wallis. 6. Oktober 2012. Web. I3. Juni 20I5. <www.imdb.com/title/

$\underline{\mathrm{ttI}} 783285 /$ reviews? start $=\mathrm{I} 8>$.

„Daniel Pall Jonasson“. „Truly a work of art.“ 20. Dezember 2009. Web. 20. Juni 2015.

$<$ www.imdb.com/title/tto $499549 /$ reviews? count $=3019 \&$ start $=99>$.

„David Bradford“. „Utterly worthless -- do not see this movie, EVER.“ Rez. von Vertical

Limit, Reg. Martin Campbell. I5. December 2000. Web. 19. Juni 20I5.

$<$ www.imdb.com/title/ttorgo865/reviews? count $=372 \&$ start $=153>$.

„dunmore_ego“. „Yes! We Have No Bananas!“ Rezension von King Kong, Reg. Peter

Jackson. 27. Dezember 2005. Web. I8. Juni 20I5. <www.imdb.com/title/ 
$\underline{\text { tto } 360717 / \text { reviews? } \text { count }=2610 \& s t a r t=1884>}>$.

„fouu2-668-459294“. „Man gets Woman, Man treats woman bad, Woman love man..“ Rez. von

Good for Nothing. Reg. Mike Wallis. 24. April 2013. Web. 13. Juni 2015.

$\leq$ www.imdb.com/title/ttı783285/reviews?start $=$ IO $>$.

„Geoff O'Callaghan“. „a lovely film.“ Rez. von The Waterborse: Legend of the Deep, Reg. Jay

Russell. 4. Mai 2008. Web. I3. Juni 2015. <www.imdb.com/title/tto760329/ reviews? start $=32>$.

„godzillageek“. „Incorrect Loch Ness facts in the movie (MILD SPOILERS).“ Foreneintrag zu The Waterhorse: Legend of the Deep, Reg. Jay Russell. 5. Januar 2008 09:05:15. Web. 13. Juni 2015. <www.imdb.com/title/tto760329/board/thread/93964628?d=93964628\#93964628 >. „Hunky Stud „. „I want to see more.“ Rez. von Without a Paddle, Reg. Steven Brill. I8. Juli 2008.

Web. 16. Juli 2015. < www.imdb.com/title/tto36475I/reviews? count $=147 \&$ start $=97>$.

„ichwan_mil“. „Vertically unlimited.“ Rez. von Vertical Limit, Reg. Martin Campbell.

30. Oktober 20I0. Web. 19. Juni 2015. <www.imdb.com/title/ttoigo865/ reviews? count $=372 \&$ start $=205>$.

„Inakaguy“. „You know what you're getting.“ Rez. von Last Samurai, Reg. Edward Zwick.

22. April 2004. Web. 19. Juni 2015. <www.imdb.com/title/tto3257Io/

reviews? count $=917 \&$ start $=19>$.

„Irishbankslave“. „Often to justify a location scout's fee.“ Foreneintrag zu The Waterhorse: Legend of the Deep, Reg. Jay Russell. 2. Dezember 2012 07:00:42. Web. I6. Juli 20I5. $<$ www.imdb.com/title/tto760329/board/nest/94499719?d=207715159\#207715159>.

„JackBluegrass“. „Oregon.“ Foreneintrag zu Without a Paddle, Reg. Steven Brill. Web.

30. März 2014 09:36:36. Web. 19. Juni 2015. <www.imdb.com/title/tto36475I/board/ thread/95822222? d=227754002\#227754002 >.

„jholmstrom-I“. „How is this film better than the Godzilla, Planet of the Apes, and other remakes?" Rezension von King Kong, Reg. Peter Jackson. 25. Dezember 2005. Web. 20. Juni 2015. <www.imdb.com/title/tto360717/reviews?count=2610\&start=I44 >.

„jimbo_4I35“. „Oregon does NOT have clear, blue rivers!“ Foreneintrag zu Without a Paddle, Reg. Steven Brill. 27. Oktober 2008 2I:50:II. Web. 19. Juni 20I5.

<www.imdb.com/title/tto36475I/board/thread/I09482769?d=I21302510\#I21302510 >. „Jmarens“. „Not filmed in Scotland.“ Foreneintrag zu The Waterhorse: Legend of the Deep, Reg. 
Jay Russell. 2I. Januar 2008 07:49:19. Web. 19. Juni 2015. <www.imdb.com/ title/tto760329/board/nest/94499719?d=95422524\#95422524>. "Jmaren5“. „Not filmed in Scotland.“ Foreneintrag zu The Waterhorse: Legend of the Deep, Reg. Jay Russell. 26. November 2008 07:00:23. Web. 19. Juni 2015. < title/tto760329/board/nest/94499719?d=I23758432\#I23758432>.

„kid_dynamo“. „A bit of sensational.“ Rez. von Vertical Limit, Reg. Martin Campbell. 26. Dezember 2000. Web. 19. Juni 2015. <www.imdb.com/title/ttorgo865/ reviews? count $=372 \&$ start $=334>$.

„markstuart-I“. „Thought this would be in the 'goofs' section (Spoilers).“ Rez. von The Waterhorse: Legend of the Deep, Reg. Jay Russell. 4. Juli 2008 16:52:58. Web. 19. Juni 2015. $<$ www.imdb.com/title/tto760329/board/thread/I076I2662?d=IIo833178\#II0833178 $>$. Messner, Reinhold. „Der K2 ist für Hollywood doch eine Nummer zu groß.“ $F A Z_{2}$ 6. Januar 200I. Web. I4. April 20I5. <www.faz.net/aktuell/sport/aufblende-der-k2-ist-fuerhollywood-doch-eine-nummer-zu-gross-II3969-p2.html>.

Mike Stone“. „It's all that you want it to be, and more.“ Rez. von Lord of the Rings: Fellowship of the Ring, Reg. Peter Jackson. 19. Dez. 200I. Web. 14. Februar 2015. <www.amazon.com/ The-Lord-Rings-Fellowship-Four-Disc/product-reviews/Boooo67DNF?pageNumber $=3>$. „nowego“. „Very enjoyable Western.“ Rez. von Good for Notbing. Reg. Mike Wallis. 26. Mai 2012. Web. I4. Februar 20I5. <www.imdb.com/title/ttI783285/reviews?start=IO $>$. „nysalor“. „A Hollow, Confused Vanilla Epic.“ Rez. von Last Samurai, Reg. Edward Zwick. 26. Januar 2004. Web. I4. Februar 2015. <www.imdb.com/title/tto325710/ reviews? count $=917 \&$ start $=19>$.

„P. Ryan 'Oregon Girl“. „YYou had to be there...." Rez. von Without a Paddle, Reg. Steven Brill. I. Oktober 2005. Web. I4. Februar 20I5. <www.amazon.com/review/

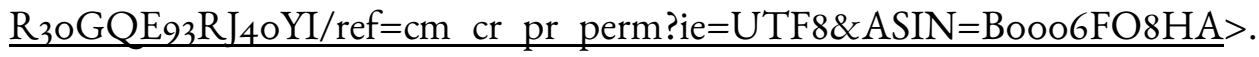
„Paul Andrews“. „”Willow, this is war not agriculture." OK fantasy adventure.“, Rez. von Willow, Reg. Ron Howard. 27. August 2005. Web. 19. Juni 2015. <www.imdb.com/ $\underline{\text { title} / \text { ttoo } 6446 / \text { reviews? count }=225 \& \text { start }=92>}>$.

„Pookyiscute“. „I want to go buy this.“ Rez. von Without a Paddle, Reg. Steven Brill. I5. Februar 2005. Web. I4. Februar 2015. <www.imdb.com/title/tto36475I/ reviews? count $=\mathrm{I} 47 \&$ start $=\mathrm{I} 43>$. 
„potato2“. „Corny.“ Rez. von Vertical Limit, Reg. Martin Campbell. 8. September 2009. Web.

I4. Februar 2015. $<$ www.imdb.com/title/ttorgo865/reviews? count $=372 \& s t a r t=153>$.

„psi“. „Eastern promise.“ Rez. von Last Samurai, Reg. Edward Zwick. 2I. Januar 2004. Web.

I4. Februar 2015. $<\underline{\text { www.imdb.com/title } / \text { tto325710 } / \text { reviews? count }=917 \& s t a r t=19}>$.

„Rhea“. „Live action Johnny quest! (but lame).“ Rez. von Vertical Limit, Reg. Martin

Campbell. ı. Dezember 2002. Web. I4. Februar 2015. <www.imdb.com/title/ ttolgo $865 /$ reviews . count $=373 \&$ start $=39>$.

„t_man_jp“. „A comment from a Japanese watcher.“ Rez. von Last Samurai, Reg. Edward Zwick. 7. Mai 2005. Web. I4. Februar 2015. <www.imdb.com/title/ $\underline{\text { tto325710/reviews? } \text { count }=917 \& \text { start }=19>}>$.

„uncreativeperson7“. „One issue with the visual effects.“ Foreneintrag zu Avatar, Reg. James Cameron. I2. Februar 20I5. Web. 20. Juni 20I5. <www.imdb.com/title/ tto $499549 / \mathrm{board} /$ thread/240228019?d=240228019\#240228019 $>$.

„villard“. „Soggy Dog Story.“ Rez. von The Waterhorse: Legend of the Deep, Reg. Jay

Russell. 20. April 2008. Web. I4. Februar 2015. <www.imdb.com/title/

tto760329/reviews? count $=87 \&$ start $=22>$.

„will7370“. „Oregon does NOT have clear, blue rivers!“ Eintrag vom 20. Juni 2008 09:I0:35.

Foreneintrag zu Without a Paddle, Reg. Steven Brill. Web.

$<$ www.imdb.com/title/tto36475I/board/thread/I09482769?d=I09482769\#I09482769 $>$.

„will7370“. „Oregon does NOT have clear, blue rivers!“ Eintrag vom 26. Juli 2008 I4:44:2I.

Foreneintrag zu Without a Paddle, Reg. Steven Brill. Web.

$<$ www.imdb.com/title/tto36475I/board/thread/I09482769?d=II3008687\#II3008687>.

„Williambulmer“. „Not filmed in Scotland.“ Foreneintrag zu The Waterhorse: Legend of the Deep,

Reg. Jay Russell. II. Januar 2008 07:26:35. Web. I4. Februar 2015.

$<$ www.imdb.com/title/tto760329/board/nest/94499719?d=94499719\#94499719>.

„wishbone_ash“. „far fetched but somewhat entertaining.“ Rez. von Vertical Limit, Reg.

Martin Campbell. 7. Juli 2003. Web. 14. Februar 2015. < www.imdb.com/

$\underline{\text { title } / \text { ttoigo865/reviews? count }=372 \& \text { start }=220}>$. 


\section{Abkürzungsverzeichnis}

Diss. $=$ Dissertation

Hrsg. $=$ Herausgeber

o.J. = ohne Jahresangabe

o.P. = ohne Paginierung

Rez. von $=$ Rezension von

Reg. $=$ Regisseur

Übers. $=$ Übersetzt von

Univ. $=$ Universität

$\mathrm{UP}=$ University Press 